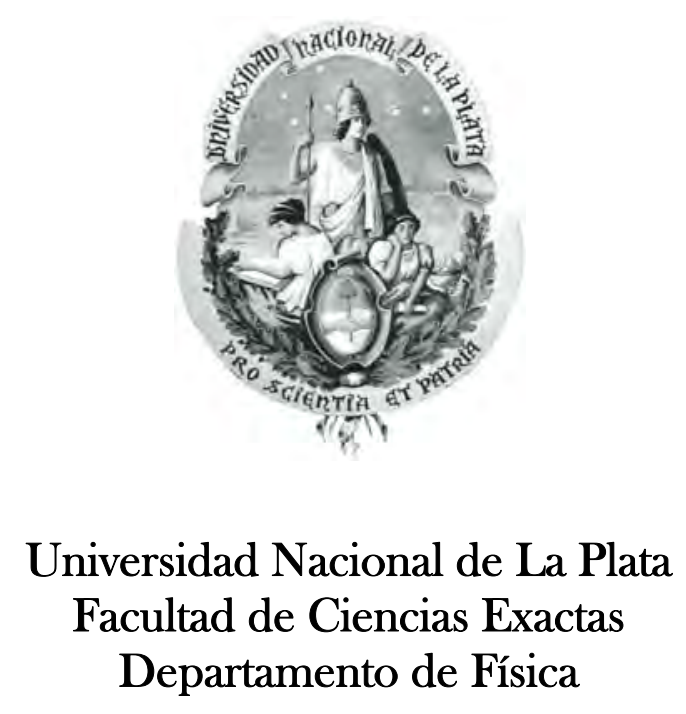

Tesis Doctoral

\title{
"Estudio Experimental y de Primeros Principios de Interacciones Hiperfinas Dinámicas en Óxidos Semiconductores Dopados con Impurezas $\left({ }^{111} \mathrm{In}(\mathrm{EC}) \rightarrow\right){ }^{111} \mathrm{Cd}$ "
}

\section{Emiliano Luis Muñoz}

Director: Prof. Dr. Mario Rentería 
Muñoz, Emiliano Luis

Estudio experimental y de primeros principios de interacciones hiperfinas dinámicas en óxidos semiconductores dopados con impurezas. - 1a ed. - La Plata : Universidad Nacional de La Plata, 2011.

E-Book.

ISBN 978-950-34-0784-4

1. Semiconductores. 2. Tesis. I. Título CDD 621.38152

Fecha de catalogación: 08/11/2011 
Estudio Experimental y de Primeros Principios de Interacciones Hiperfinas Dinámicas en Óxidos

Semiconductores Dopados con Impurezas $\left({ }^{111} \operatorname{In}(\mathrm{EC}) \rightarrow\right)^{111} \mathrm{Cd}$

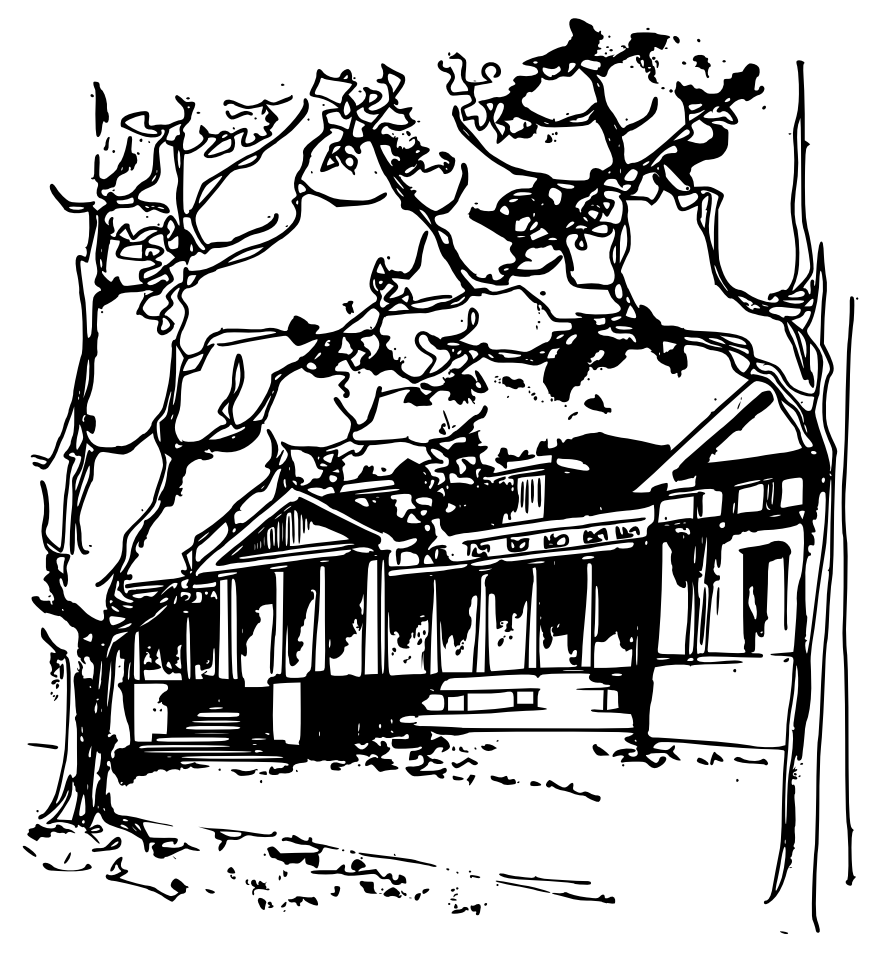

Emiliano Luis Muñoz 


\section{Tesis Doctoral}

Realizada en el

Departamento de Física

Presentada en la

Facultad de Ciencias Exactas

Universidad Nacional de La Plata

Para obtener el Título de

Doctor de la Facultad de Ciencias Exactas

\section{Por \\ Emiliano Luis Muñoz}

Título de la Tesis

Estudio Experimental y de Primeros Principios de Interacciones Hiperfinas Dinámicas en Óxidos

Semiconductores Dopados con Impurezas $\left({ }^{111} \operatorname{In}(E C) \rightarrow\right){ }^{111} \mathrm{Cd}$

Director: Prof. Dr. Mario Rentaría

Jurado de Tesis:

Prof. Dra. Helena M. Petrilli

Prof. Dra. Graciela Punte

Prof. Dr. Artur W. Carbonari 


\section{INDICE}

\section{CAPÍTULO 1 Introducción}

CAPÍTULO 2 Correlaciones angulares

2-1 Función de Correlación $\quad 7$

$\begin{array}{lll}2-2 & \text { Factor de Perturbación } & 10\end{array}$

2-3 Interacción cuadrupolar eléctrica 12

2-4 Método experimental 17

2-4-1 Medida de las Correlaciones Angulares Perturbadas

$\begin{array}{ll}\text { Diferenciales en Tiempo } & 17\end{array}$

2-4-2 Equipo experimental 24

2-4-3 La sonda PAC $\left({ }^{111} \mathrm{In} \rightarrow\right)^{111} \mathrm{Cd} \quad 27$

CAPÍTULO 3 Interacciones hiperfinas dinámicas 31

3-1 Comentarios sobre Interacciones Hiperfinas Dinámicas 31

3-2 Modelos de "after effects" 32

3-2-1 Modelo de Bäverstam y Othaz 32

3-2-2 Modelo de Relajación Electrónica Unidireccional 34

CAPÍTULO 4 Métodos de cálculo 37

4-1 Modelo de cargas puntuales 37

4-2 Teoría de la Funcional Densidad 39

4-2-1 Método APW 44

4-2-2 Método FP-LAPW 46

4-2-3 Método FP-LAPW+LO 49

4-2-4 Método APW+lo 50

4-3 Metodología del cálculo FP-APW+lo para el caso de impurezas en semiconductores 52 


\section{CAPÍTULO 5 Sistemas estudiados}

5-1 Sesquióxidos con estructura bixbita: $\mathrm{Sc}_{2} \mathrm{O}_{3}, \mathrm{Y}_{2} \mathrm{O}_{3}$ e $\mathrm{In}_{2} \mathrm{O}_{3} \quad 59$

5-2 El dióxido de Estaño $\left(\mathrm{SnO}_{2}\right) \quad 61$

5-3 El monóxido de Estaño (SnO) 62

5-4 El monóxido de Zinc (ZnO) 63

5-5 Preparación de las muestras $\quad 64$

\section{CAPÍTULO 6 Resultados experimentales 69}

6-1 Resultados PAC del óxido SnO medido con la sonda $\left({ }^{111} \mathrm{In} \rightarrow\right)^{111} \mathrm{Cd} \quad 69$

6-1-1 Resultados PAC del $1^{\circ}$ experimento en SnO: $\left({ }^{111} \mathrm{In} \rightarrow\right)^{111} \mathrm{Cd} \quad 69$

6-1-2 Comparación entre el $1^{\circ}$ y el $2^{\circ}$ experimento 71

6-2 Resultados en las bixbitas $\mathrm{Sc}_{2} \mathrm{O}_{3}, \mathrm{In}_{2} \mathrm{O}_{3}$ e $\mathrm{Y}_{2} \mathrm{O}_{3}$ medidas con la sonda $\left({ }^{111} \mathrm{In} \rightarrow\right)^{111} \mathrm{Cd}$

6-2-1 Resultados PAC en $\operatorname{In}_{2} \mathrm{O}_{3}:\left({ }^{111} \mathrm{In} \rightarrow\right)^{111} \mathrm{Cd}$

6-2-2 Resultados PAC en $\mathrm{Sc}_{2} \mathrm{O}_{3}:\left({ }^{111} \mathrm{In} \rightarrow\right)^{111} \mathrm{Cd}$

6-2-3 Resultados PAC en $\mathrm{Y}_{2} \mathrm{O}_{3}:\left({ }^{111} \mathrm{In} \rightarrow\right)^{111} \mathrm{Cd}$

6-3 Resultados PAC del óxido $\mathrm{SnO}_{2}$ medido con la sonda $\left({ }^{111} \mathrm{In} \rightarrow\right)^{111} \mathrm{Cd}$

6-4 Resultados PAC del óxido $\mathrm{ZnO}$ medido con la sonda $\left({ }^{111} \mathrm{In} \rightarrow\right){ }^{111} \mathrm{Cd} \quad 88$

\section{CAPÍTULO 7 Cálculos FP-APW+lo 93}

7-1 Cálculos $a b$ initio en los sistemas puros 93

7-1-1 Cálculos en los óxidos $\mathrm{In}_{2} \mathrm{O}_{3}, \mathrm{Y}_{2} \mathrm{O}_{3}$ y $\mathrm{Sc}_{2} \mathrm{O}_{3}$ puros 93

7-1-2 Cálculos en ZnO puro 99

7-1-3 Cálculos en $\mathrm{SnO}_{2}$ puro 101

7-1-4 Cálculos en SnO puro 103

7-2 Cálculos ab initio en sistemas dopados 105

7-2-1 Comparación de cálculos en $\mathrm{Sc}_{2} \mathrm{O}_{3}$ :Cd con celda relajada y no relajada 
7-2-2 Estudio de la convergencia de parámetros hiperfinos en los sistemas dopados

7-2-3 Cálculos APW+lo del tensor GCE en función del estado

de carga de la celda en óxidos con estructura bixbita

7-2-4 Cálculos APW+lo del tensor GCE en ZnO dopado con Cd

7-2-5 Cálculos APW+lo del tensor GCE en $\mathrm{SnO}_{2}$ dopado con Cd

7-2-6 Cálculos APW+lo del tensor GCE en SnO dopado con Cd

\section{CAPÍTULO 8 Comparación de resultados y discusión}

8-1 Comparación de resultados PAC con predicciones APW+lo en los óxidos con estructura bixbita

8-1-1 Comparación de resultados en $\mathrm{In}_{2} \mathrm{O}_{3}:{ }^{111} \mathrm{Cd}$

8-1-2 Comparación de resultados en $\mathrm{Sc}_{2} \mathrm{O}_{3}:{ }^{111} \mathrm{Cd}$

8-1-3 Comparación de resultados en $\mathrm{Y}_{2} \mathrm{O}_{3}:{ }^{111} \mathrm{Cd}$ 146

8-2 Comparación de resultados PAC con cálculos APW+lo en $\mathrm{SnO}_{2}:{ }^{111} \mathrm{Cd}$

8-3 Comparación de resultados PAC con cálculos APW+lo en $\mathrm{ZnO}:{ }^{111} \mathrm{Cd}$ 152

8-4 Comparación de resultados PAC con cálculos APW+lo en SnO: ${ }^{111} \mathrm{Cd}$ 155

8-5 Localización de los estados electrónicos del nivel de impureza 157 8-5-1 Localización electrónica en el óxido $\mathrm{In}_{2} \mathrm{O}_{3}: \mathrm{Cd}$

8-5-2 Localización electrónica en el óxido $\mathrm{Y}_{2} \mathrm{O}_{3}$ :Cd 162

8-5-3 Localización electrónica en el óxido $\mathrm{Sc}_{2} \mathrm{O}_{3}$ :Cd 164

8-5-4 Localización electrónica en el óxido $\mathrm{SnO}_{2}$ : $\mathrm{Cd} \quad 167$

8-5-5 Localización electrónica en el ZnO:Cd 170

8-5-6 Localización electrónica en el SnO:Cd 175

8-6 Modelo semi-empírico - ab initio de interacciones hiperfinas dinámicas 179 8-6-1 Modelo semi-empírico - ab initio aplicado al caso de los óxidos con estructura bixbita

8-6-2 Modelo semi-empírico - ab initio aplicado al óxido $\mathrm{SnO}_{2}$

8-6-3 Modelo semi-empírico - ab initio aplicado al óxido SnO 183

8-6-4 Modelo semi-empírico - ab initio aplicado al óxido ZnO 184

8-7 Análisis de las relajaciones estructurales 185 
CAPÍTULO 9 Conclusiones

APENDICE I

$\begin{array}{lll}\text { I-1 } & \text { Formalismo de la matriz densidad } & 197\end{array}$

I-2 Función de correlación angular no perturbada para $I=1 \quad 203$

$\begin{array}{ll}\text { APENDICE II } & 207\end{array}$

II-1 Modelo de Bäverstam y Othaz 207

$\begin{array}{ll}\text { APENDICE III } & 209\end{array}$

III-1 Cálculos PCM en óxidos con estructura bixbita 209

III-2 Cálculos PCM en los óxidos $\mathrm{ZnO}, \mathrm{SnO}$ y $\mathrm{SnO}_{2}$. 210

$\begin{array}{ll}\text { Publicaciones } & 211\end{array}$

$\begin{array}{lr}\text { Agradecimientos } & 215\end{array}$ 


\section{Capítulo No 1}

\section{Introducción}

La inclusión de impurezas en semiconductores tiene una gran importancia en el campo de la física clásica como así también en física aplicada. En efecto, la determinación de las propiedades estructurales y electrónicas producidas por la inclusión de los átomos-impurezas en la red del semiconductor presentan un gran interés tecnológico. Recientemente, el control de los estados de impureza introducidos en el gap del semiconductor ha motivado estudios basados en cálculos ab initio de estructura electrónica en semiconductores dopados [Ahmad 2006]. Por otro lado, la correcta caracterización de las energías de los estados de impureza, del tipo de portadores de carga inducidos y la buena determinación de la vida media de los portadores ha cobrado gran importancia en el campo experimental [Müller 2007].

Las técnicas experimentales basadas en la determinación de la interacción hiperfina entre un núcleo-sonda y el campo extranuclear circundante son herramientas muy útiles para el estudio del entorno subnanoscópico del átomo-sonda (generalmente una impureza en el sistema bajo estudio). Cabe destacar que las determinaciones experimentales de observables en sitios de impureza predichos por cálculos de primeros principios son fundamentales para evaluar la aplicación y confiabilidad de métodos de cálculos $a b$ initio utilizados en el estudio de propiedades estructurales y electrónicas producidas por la inclusión de impurezas metálicas en semiconductores. El tensor Gradiente de Campo Eléctrico (GCE) resulta ser un observable muy sensible frente a cambios locales de la densidad electrónica de carga en el entorno subnanoscópico del núcleo-sonda donde el GCE es determinado, debido a la dependencia funcional con $r^{-3}$ desde la carga que lo produce. Actualmente, el tensor GCE puede ser determinado con gran precisión en prácticamente cualquier material utilizando la técnica hiperfina de las Correlaciones Angulares Perturbadas $\gamma-\gamma$ Diferenciales en Tiempo (PAC), a partir de la medida de la interacción cuadrupolar eléctrica entre el momento cuadrupolar eléctrico de un núcleo-sonda, adecuadamente introducido en el sólido, y el tensor GCE del campo extranuclear. 
Para la correcta interpretación de la física subyacente en los modelos fenomenológicos utilizados para analizar los resultados experimentales determinados con la técnica PAC son necesarios cálculos $a b$ initio de estructura electrónica de los sistemas abordados. En los últimos años, este tipo de cálculos han sido aplicados exitosamente al estudio de semiconductores dopados con impurezas metálicas [Settels 1999, Lany 2000, Jeong 2001] y tamién en sistemas intermetálicos [Terrazos 2002]. Recientemente, el doble abordaje experimental-ab initio basado en la utilización del GCE como herramienta eficaz para investigar propiedades estructurales y electrónicas de impurezas metálicas localizadas sustitucionalmente en sitios de catión en óxidos semiconductores ha sido demostrado con éxito [ver, por ejemplo, Errico 2003, Errico 2007, Alonso 2008, Darriba 2009]. Por otro lado, el uso de cálculos de primeros principios para soportar la física de modelos fenomenológicos utilizados en el ajuste de medidas experimentales se ha comenzado a aplicar satisfactoriamente recientemente en algunos sistemas, como en el caso del $\mathrm{Sc}_{2} \mathrm{O}_{3}:{ }^{111} \mathrm{Cd}$ [Muñoz 2011].

El objetivo más importante de esta Tesis es obtener un modelo semi-empírico - $a b$ initio que describa las interacciones hiperfinas dinámicas observadas por sondasimpurezas $\left({ }^{111} \mathrm{In} \rightarrow\right)^{111} \mathrm{Cd}$ localizadas sustitucionalmente en sitios de catión en óxidos semiconductores. Estas interacciones son atribuidas al proceso dinámico, que tiene lugar en el entorno sub-nanoscópico del átomo-sonda, y posterior al decaimiento por captura electrónica (EC, por electron capture) del isótopo padre ${ }^{111} \operatorname{In}\left((\mathrm{EC}) \rightarrow{ }^{111} \mathrm{Cd}\right)$. Este proceso en la literatura se lo conoce como after-effects (o AE) debido a que se produce luego de la EC de un electrón de la capa K del ${ }^{111} \mathrm{In}$. En el comienzo del after-effects, el núcleo ${ }^{111}$ In deja a la sonda ${ }^{111} \mathrm{Cd}$ en un estado cargado positivamente y en una situación energéticamente lejana del equilibrio. Entonces, sondas con algunos huecos electrónicos extras $\left(\mathrm{Cd}^{+3}\right.$ o $\left.\mathrm{Cd}^{+4}\right)$ con respecto al estado iónico de equilibrio en el sólido $\left(\mathrm{Cd}^{+2}\right)$ pueden permanecer con dicha configuración electrónica un tiempo suficiente para alcanzar la ventana temporal de la medida PAC. Este tiempo es el que transcurre durante las emisiones de las radiaciones $\gamma_{1}$ y $\gamma_{2}$ de la cascada $\gamma-\gamma$, mediante la cual el ${ }^{111} \mathrm{Cd}$ se desexcita llegando a su estado fundamental. Durante esta ventana temporal ocurrirán transiciones electrónicas al estado más estable, las cuales dependerán de las características electrónicas de la red huésped (movilidad y disponibilidad electrónica, por ejemplo). Estas transiciones modifican en función del tiempo la distribución de carga en las proximidades de la sonda, dando lugar a procesos dinámicos (usualmente 
como "relajaciones dinámicas”) que pueden ser monitoreados por medio de técnicas hiperfinas adecuadas.

Desde el punto de vista experimental, en el presente trabajo se midió la dependencia del GCE en el sitio de la sonda-impureza $\left({ }^{111} \operatorname{In}(\mathrm{EC}) \rightarrow\right)^{111} \mathrm{Cd}$ como función de la temperatura utilizando la técnica PAC, en sistemas que eran candidatos a presentar atenuación de carácter electrónico en sus espectros, o en los que ya se había observado interacciones dinámicas [Muñoz 2007, Bibiloni 1984, Habenicht 1996, Bartos 1991, Wolf 1986]. Las medidas experimentales con presencia de after-effects fueron ajustadas en este trabajo con el modelo de interacciones hiperfinas dinámicas de Bäverstam y Othaz [Bäverstam 1972], basado en el trabajo pionero de Abragam y Pound [Abragam 1953]. Cabe destacar que este modelo comenzó a utilizarse en el análisis de los espectros PAC con relajaciones dinámicas a principios de 1980 por el grupo de La Plata [Bibiloni 1984, Massolo 1986] y siguió siendo utilizado con frecuencia hasta la actualidad (ver, por ejemplo [Sato 2008]). El modelo de Baverstäm y Othaz supone que si la sonda ${ }^{111} \mathrm{Cd}$ llega al estado de equilibrio durante la ventana temporal de la medida PAC entonces la interacción hiperfina entre el núcleo-sonda y el campo extranuclear devendrá estática. Este modelo fenomenológico reprodujo correctamente los resultados experimentales con presencia de AE (en aquellos sistemas donde fue aplicado) a través de los factores de perturbación propuestos por el modelo. Sin embargo, la literatura carece de una interpretación teórica basada en cálculos de estructura electrónica a partir de primeros principios que describa la presencia del GCE dependiente del tiempo con las propiedades de la densidad electrónica perturbada en el entorno del sitio del Cd. En esta Tesis nos proponemos brindar una posible descripción de este fenómeno a través de un estudio de primeros principios de la dependencia del GCE (observado en el sitio del Cd) con el estado de carga de la celda analizando las propiedades de la densidad electrónica perturbada en el entorno del sitio de la impureza Cd.

En el plano teórico de esta Tesis se empleó el método Full-Potential Augmented Plane Wave plus local orbital (FP-APW+lo), a través de su implementación en el código Wien2k, para la realización de cálculos de estructura electrónica y del GCE en los óxidos dopados. La dilución de las impurezas fue simulada empleando el método de la supercelda, en el cual se construye una celda de tamaño mayor a la celda unidad y donde uno de los cationes es reemplazado por el átomo-sonda. A través de un proceso de libre minimización de las fuerzas “cuánticas” en los átomos de la celda se determinaron las posiciones finales de equilibrio de los mismos. Se utilizaron las dos 
aproximaciones usuales para el término de correlación e intercambio, la Local-Density Approximation (LDA) y la Generalizad-Gradient Approximation (GGA) y se estudió su aplicabilidad a cada sistema. También se aplicó la nueva parametrización para el término de correlación e intercambio, CW-GGA, que ha demostrado ser más eficiente a las antes mencionadas para el estudio de óxidos metálicos. Se estudió también el estado de carga de la celda en forma autoconsistente, variando la carga del sistema teniendo en cuenta si las impurezas son donoras y/o aceptoras según sea el óxido en estudio, comparando las predicciones teóricas del GCE con los resultados experimentales. Además del cálculo del tensor GCE (magnitud, orientación y signo de sus componentes principales), se estudió la densidad de estados del sistema, la existencia de niveles de impureza, el carácter de los mismos, la localización espacial de los estados electrónicos cercanos al nivel de Fermi, las relajaciones estructurales producidas por la inclusión de la impureza en la red del huésped y las distintas contribuciones al GCE.

Los sistemas abordados en la presente Tesis son: los sesquióxidos $\mathrm{Sc}_{2} \mathrm{O}_{3}, \mathrm{Y}_{2} \mathrm{O}_{3}$ e $\mathrm{In}_{2} \mathrm{O}_{3}$; los óxidos de estaño $\mathrm{SnO}$ y $\mathrm{SnO}_{2}$, y el óxido $\mathrm{ZnO}$. Los cationes que conforman los tres sesquióxidos con estructura bixbita presentan la particularidad de tener la capa electrónica cerrada y sus respectivas medidas PAC presentan una importante atenuación dinámica. En el óxido $\mathrm{SnO}_{2}$ con estructura rutilo se han caracterizado dos interacciones dinámicas a pesar de que su estructura cristalina tiene un único sitio inequivalente de catión, siendo el fenómeno de AE observado muy apreciable. Finalmente, en los óxidos $\mathrm{SnO}$ y $\mathrm{ZnO}$ el ión $\mathrm{Cd}^{2+}$ presenta la particularidad de ser isovalente con los cationes $\mathrm{Sn}^{2+}$ y $\mathrm{Zn}^{2+}$ en sus respectivos óxidos. Sin embargo, en el monóxido de estaño se ha observado una importante atenuación dinámica, mientras que en el monóxido de Zinc la atenuación parece ser prácticamente despreciable. A partir del excelente acuerdo que surge de la buena comparación entre los resultados experimentales y las predicciones de los cálculos $a b$ initio, se elaboró un modelo experimental y de primeros principios que describe el proceso dinámico posterior al decaimiento por EC del núcleo-sonda y que da lugar a las interacciones hiperfinas dinámicas observadas por la sonda-impureza $\left({ }^{111} \mathrm{In} \rightarrow\right)^{111} \mathrm{Cd}$ localizada sustitucionalmente en los sitios catiónicos de los óxidos semiconductores abordados.

En el Capítulo 2 se presenta la teoría de las Correlaciones Angulares Perturbadas Diferenciales en Tiempo y la técnica PAC. 
En el Capítulo 3 se describen los modelos utilizados en el análisis de los espectros PAC con presencia de interacciones hiperfinas dinámicas.

En el Capítulo 4 se describen los métodos teóricos utilizados en este trabajo para calcular las propiedades estructurales y electrónicas y, en particular, el tensor GCE en el sitio de la impureza.

En el Capítulo 5 se detallan las características de los óxidos estudiados y la preparación de las muestras.

En el Capítulo 6 se analizan los resultados experimentales obtenidos en cada sistema.

En el Capítulo 7 se describen los resultados de los cálculos ab initio obtenidos en cada óxido abordado.

En el Capítulo 8 se comparan los resultados experimentales con las predicciones teóricas del GCE, se discuten las propiedades estructurales y electrónicas en cada semiconductor dopado y se elabora un modelo de interacciones hiperfinas dinámicas basado en medidas experimentales y cálculos ab initio.

Finalmente, se presentan las conclusiones de esta Tesis.

\section{Referencias}

[Ahmad 2006] S. Ahmad, K. Hoang, and S.D. Mahanti, Phys. Rev. Lett. 96, 056403 (2006).

[Müller 2007] S. Müller, D. Stichtenoth, M. Uhrmacher, H. Hofsäss, C. Ronninga and J. Röder, Appl. Phys. Lett. 90, 012107 (2007).

[Settels, 1999] A. Settels, T. Korhonen, N. Papanikolaou, R. Zeller, and P.H. Dederichs, Phys. Rev. Lett. 83, 4369 (1999).

[Lany, 2000] Stephan Lany, P. Blaha, J. Hamann, V. Ostheimer, H. Wolf, and T. Wichert, Phys. Rev. B. 62, R2259 (2000).

[Jeong, 2001] S. Jeong and A. Oshiyama, Phys. Rev. Lett. 86, 3574 (2001).

[Terrazos 2002] L.A. Terrazos, H.M. Petrilli, M. Marszalek, H. Saitovitch, P.R.J. Silva, P. Blaha and K. Schwarz, Solid. State Comunn. 121, 525 (2002).

[Errico, 2002] L. A Errico, G. Fabricius, M. Rentería, P. De La Presa y M.Forker, Phy. Rev. Lett 89, 55503 (2002).

[Errico 2007] ; L.A. Errico, M. Rentería, H.M. Petrilli, Phys. Rev. B 75155209 (2007).

[Darriba 2009] G.N. Darriba, L.A. Errico, P.D. Eversheim, G. Fabricius, and M. Rentería, Phys. Rev. B 79, 115213 (2009). 
[Alonso 2008] R.E. Alonso, L.A. Errico, E.L. Peltzer y Blancá, A. López-García, A. Svane, and N.E. Christensen, Phys. Rev. B 78, 165206 (2008).

[Muñoz 2011] E.L. Muñoz, D. Richard, A.W. Carbonari, L.A. Errico and M. Rentería, Hyperfine Interact. 197, 199 (2011).

[Muñoz 2007] E.L. Muñoz, A.W. Carbonari, L.A. Errico, A.G. Bibiloni, H. M. Petrilli, M. Rentería, Hyp. Int. 17837 (2007).

[Bibiloni 1984] A.G. Bibiloni, J. Desimoni, C.P. Massolo, L.A. Mendoza-Zelis, A.F. Pasquevich, F.H. Sanchez y A. Lopez-García, Phys. Rev. B 29, 1109 (1984).

[Habenicht 1996] S. Habenicht, D. Lulascu, M. Uhrmacher, L. Ziegeler. K.P. Lieb y ISOLDE collaboration, Z. Phys. B 101, 187 (1996).

[Bartos 1991] A. Bartos, K.P. Lieb, A.F. Pasquevich, M. Uhrmacher e ISOLDE collaboration, Phys. Lett. A 157, 513 (1991).

[Wolf 1986] Wolf, H., Deubler, S., Forkel, D., Foettinger, H., Iwatschenko-Borho, M., Meyer, M., Renn, M., Witthuhn, W., Mat. Sci. Forum. 10-12, 863-868 (1986).

[Bäverstam 1972] U. Bäverstam, R. Othaz, N. De Sousa and B. Ringström, Nucl. Phys. A186, 500 (1972).

[Abragam 1953] A. Abragam and R. V. Pound, Phys. Rev. 92, 943 (1953).

[Massolo 1986] C. P. Massolo, J. Desimoni, A.G. Bibiloni, L.A. Mendoza-Zélis, L.C. Damonte, A. R. Lopez-Garcia, P.W. Martin, S.R. Dong, and J.G. Hooley, Phys. Rev. B 12, 8857 (1986).

[Sato 2008] W. Sato, Y. Itsuki, S. Morimoto, H. Susuki, S. Nasu, A. Shinohara, and Y. Ohkubo, Phys. Rev. B 78045319 (2008). 


\section{Capítulo $\mathbf{N}^{0} 2$}

\section{Correlaciones angulares}

En el presente Capítulo se realiza una descripción sintetizada de la teoría de las Correlaciones Angulares Perturbadas Diferenciales en Tiempo. Se aborda el caso particular de la interacción cuadrupolar eléctrica entre el momento cuadrupolar del núcleo y el Gradiente de Campo Eléctrico del campo extranuclear y por último se describe la metodología experimental utilizada en el presente trabajo. Para un análisis más exhaustivo del tema se recomienda ir al Apénice I y al trabajo [Frauenfelder, 1968].

\section{2-1 Función de correlación}

La desexcitación de un isótopo radiactivo debida a la emisión consecutiva de dos radiaciones $\gamma$ está caracterizada por tres estados nucleares: un estado inicial de momento angular $I_{i}$, un estado intermedio $I$ (el cual tiene una vida media $\lambda$ y un momento cuadrupolar Q) y un estado final $I_{f}$. Los núcleos decaen del estado $I_{i}$ al estado $I$ emitiendo la primera radiación $\gamma_{1} \mathrm{y}$ acceden al estado final emitiendo una segunda radiación $\gamma_{2}$ (ver, por ejemplo, la sección 2 del Apéndice I). Este proceso de denomina “cascada $\gamma-\gamma$ ” y se esquematiza en la Figura 2.1.

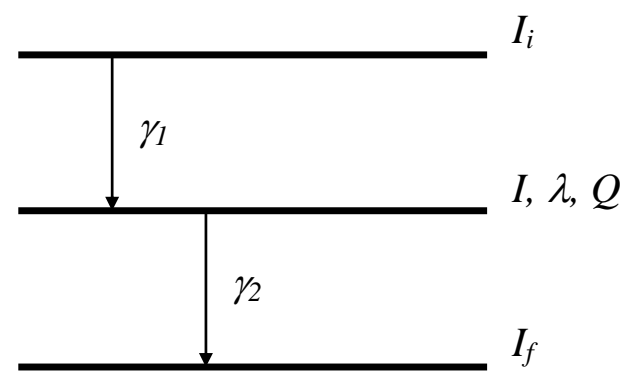

Figura 2.1: Esquema de desexcitación de un núcleo radiactivo a través de una cascada $\gamma-\gamma$. 
Entre las direcciones de emisión $\boldsymbol{k}_{\mathbf{1}}$ y $\boldsymbol{k}_{\mathbf{2}}$ de las radiaciones $\gamma_{1}$ y $\gamma_{2}$, respectivamente, existe una correlación debido a la conservación del momento angular del sistema. La orientación del espín nuclear del estado intermedio I está relacionada con la dirección $\boldsymbol{k}_{\mathbf{1}}$ y puede expresarse en términos del número cuántico momento angular magnético $m$ respecto a un eje de cuantificación arbitrario que en nuestro caso será $\boldsymbol{k}_{\mathbf{1}}$.

Debido a que una muestra radiactiva está compuesta por un gran número de núcleos realizando transiciones idénticas y teniendo en cuenta que sus espines se encuentran orientados aleatoriamente, entonces no habrá una dirección preferencial de emisión por lo que el patrón deviene isótropo. Para “romper” esta isotropía debemos lograr que los núcleos estén orientados en alguna dirección preferencial. La detección de $\gamma_{1}$ en una dirección $\boldsymbol{k}_{\mathbf{1}}$ selecciona un subconjunto de núcleos particularmente orientados, por lo que la radiación $\gamma_{2}$ detectada en coincidencia con $\gamma_{1}$ tendrá una distribución angular anisotrópica definida.

En presencia de campo extranuclear, por ejemplo el campo generado en un sólido cristalino, los momentos nucleares interactuarán con dicho campo. Esta interacción se conoce como interacción hiperfina y es capaz de producir (semi-clásicamente) la precesión de los espines nucleares alrededor de alguna dirección preferencial, cambiando el patrón de emisión. Si el campo cristalino solo tiene una contribución eléctrica, entonces, el gradiente del campo eléctrico (GCE) interactúa con el momento cuadrupolar eléctrico nuclear. Para una diferencia de tiempo nula entre las emisiones de $\gamma_{1} \mathrm{y} \gamma_{2}$, la diferencia de contaje entre las coincidencias medidas entre el detector 2 (donde se detecta $\gamma_{2}$ ) y el detector 1 (donde se detecta $\gamma_{1}$ ) posicionados a $90^{\circ}$ y $180^{\circ}$ nos da la anisotropía (ver Figura 2.2). En el caso de no tener una emisión simultánea de las radiaciones, la anisotropía será función del tiempo transcurrido entre la emisión de $\gamma_{1}$ y de $\gamma_{2}$ debido a que durante este tiempo el espín del estado intermedio nuclear precesa alrededor del eje de cuantificación. La anisotropía se verá recuperada para un tiempo $\Delta t=2 \pi / \omega$, donde $\omega$ es la frecuencia de precesión de espín. Esta frecuencia es proporcional a la componente principal del tensor GCE y está relacionada con la separación de los subestados $m$ del nivel intermedio $I$ cuando el campo externo rompe su degeneración. 


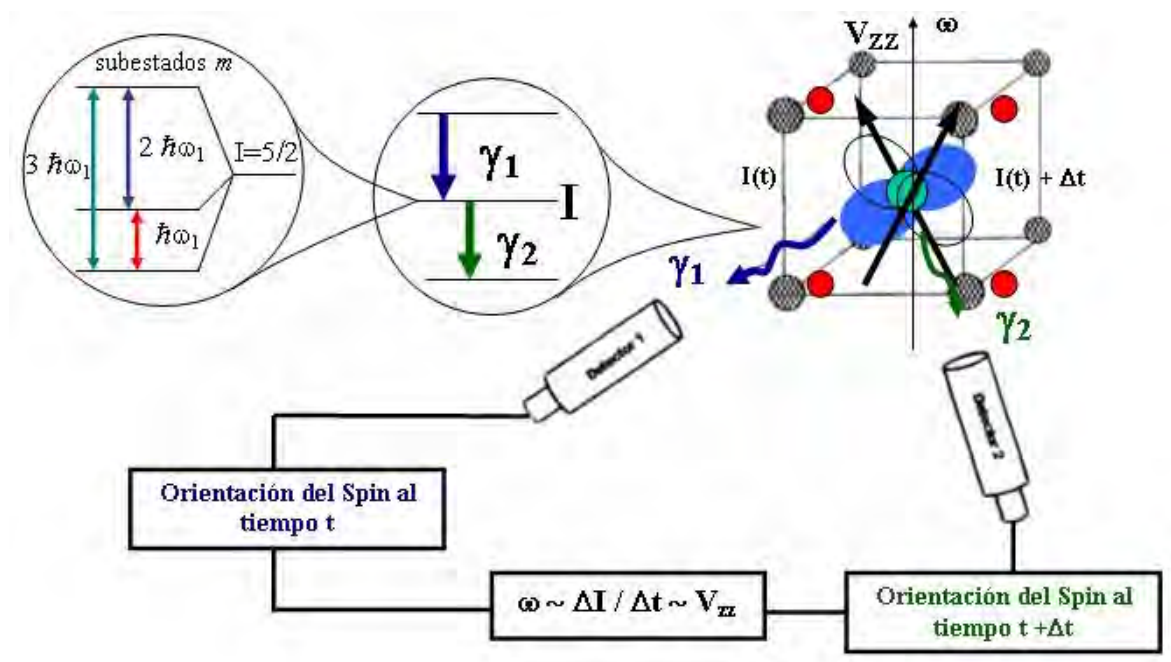

Figura 2.2: Esquema semi-clásico del principio básico de la determinación de la frecuencia de precesión del espín nuclear debido a la presencia de un campo extranuclear.

La relación entre la frecuencia de precesión $\omega$ y el desdoblamiento $\Delta E$ se conoce como precesión de Larmor y es $\omega=\Delta E / \hbar$. La Figura 2.3 muestra el desdoblamiento de un estado nuclear para el caso particular de $I=5 / 2$ y sus energía en función de la frecuencia de Larmor.

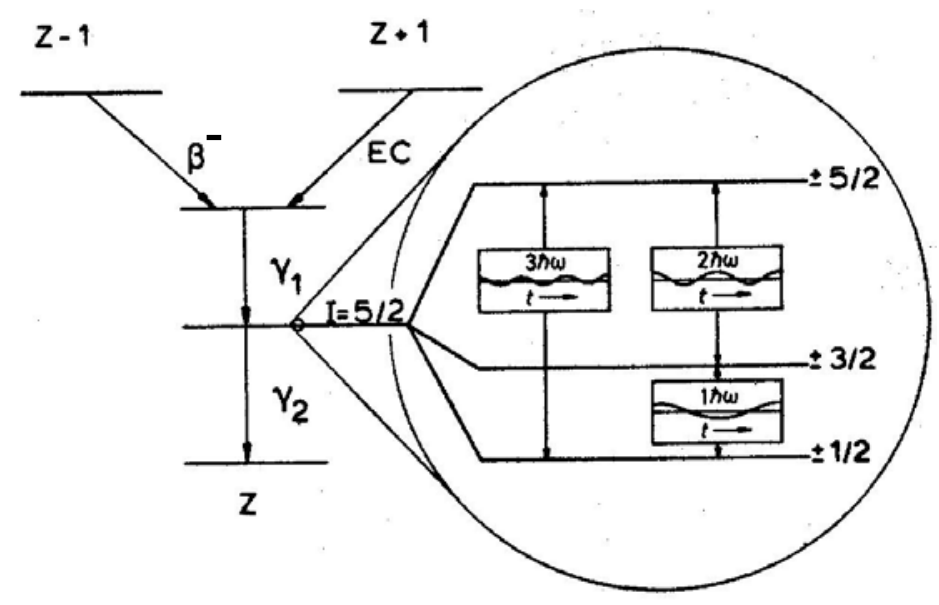

Figura 2.3: Esquema de decaimiento nuclear de un núcleo a través de la emisión consecutiva de dos radiaciones $\gamma_{1}$ y $\gamma_{2}$ y desdoblamiento del nivel intermedio I de la cascada $\gamma-\gamma$. 
La probabilidad de que $\gamma_{2}$ sea emitido en la dirección $\boldsymbol{k}_{\mathbf{2}}$ en una abertura $d \Omega_{2}$ al tiempo $t+d t$, si $\gamma_{1}$ ha sido emitido en la dirección $\boldsymbol{k}_{1}$ en el instante $t$ en una abertura $d \Omega_{1}$ es:

$$
P\left(\vec{k}_{1}, \vec{k}_{2}, t\right)=W\left(\vec{k}_{1}, \vec{k}_{2}, t\right) \cdot e^{-t / \lambda} d \Omega_{1} d \Omega_{2} d t
$$

donde $W\left(\vec{k}_{1}, \vec{k}_{2}, t\right)$ se define como la función de correlación angular y es la probabilidad por unidad de ángulo sólido $d \Omega_{1}$ y $d \Omega_{2}$, y por unidad de tiempo de que $\gamma_{1}$ se emita en la dirección $\boldsymbol{k}_{\mathbf{1}}$ al tiempo $t$ en correlación con la emisión de $\gamma_{2}$ en la dirección $\boldsymbol{k}_{\mathbf{2}}$ al tiempo $t+d t$.

Como se demuestra en el Apéndice I, la función de correlación no perturbada puede expresarse como función del ángulo entre las direcciones de emisión $\theta$ como sigue:

$W(\theta)=\sum_{K=0}^{K_{\text {máx }}} A_{K K} P_{K}(\cos (\theta))$,

donde $K=0,2,4, \ldots, K_{\text {máx }}$ y $K_{\text {máx }}=\left\{2 I, 2 L_{1}, 2 L_{2}\right\}, L_{i}$ es el momento angular de $\gamma_{i}$. $P_{K}(\cos (\theta))$ es el $K$-ésimo polinomio de Legendre, y los coeficientes $A_{K K}$, denominados coeficientes direccionales o coeficientes de anisotropía, miden la desviación respecto del caso isotrópico $(W(\theta)=1)$. La normalización de esta expresión se realiza tomando $A_{00}=1$ y como puede apreciarse en (2.2), para obtener un patrón anisotrópico se requiere $I \geq 1$. Cabe destacar que debido a que la probabilidad de transición entre estados nucleares decrece con el incremento del momento angular de la radiación emitida, en general $K_{\text {máx }}$ no es mayor que 4.

\section{2-2 Factor de Perturbación}

Si consideramos el efecto de los campos externos al núcleo, entonces tendrá lugar el desdoblamiento de los subestados $m$ degenerados del estado intermedio de espín $I$. Desde un punto de vista semi-clásico la interacción hiperfina entre el tensor GCE y el momento cuadrupolar $Q$ de un núcleo produce un movimiento de precesión del espín nuclear alrededor del eje de cuantificación sobre el cual cada subestado tiene una proyección $m$ dando lugar a una re-orientación nuclear. El desdoblamiento típico de los 
níveles de energía $\triangle E$ es del orden de $10^{-8}-10^{-6} \mathrm{eV}$, dependiendo de la intensidad del campo. Este desdoblamiento es varios ordenes de magnitud menor que las energías involucradas en las transiciones nucleares que son del orden de $10^{2} \mathrm{keV}$.

Finalmente, estos efectos perturbativos modifican la orientación del espín nuclear y cuantitativamente también se modifica la función de correlación angular, siendo ahora función del ángulo $\theta$ y del tiempo $t$ (ver sección 1 del Apéndice I), tomando la forma:

$$
W(\theta, t)=\sum_{K=0}^{K_{\max }} A_{K K} G_{K K}(t) P_{K}(\cos (\theta))
$$

donde $G_{K K}(t)$ se denomina Factor de Perturbación y contiene toda la información de la interacción hiperfina entre el tensor GCE del campo extranuclear y el momento cuadrupolar del núcleo.

La forma explícita del factor de perturbación depende del tipo de interacción hiperfina experimentada por el núcleo (por ejemplo interacción dipolar magnética o interacción cuadrupolar eléctrica), también depende de la dependencia temporal de dicha interacción (si es estática o dinámica), como así también del tipo de muestra (sistemas en estado bulk, monocristales, nanopartículas, etc). A modo de ejemplo, mostramos el caso del factor de perturbación para una muestra policristalina donde el núcleo experimenta una interacción cuadrupolar eléctrica con un campo externo estático, esto es, independiente del tiempo.

$$
G_{K K}(t)=\sum_{n=0}^{n_{m a x}} S_{K n} \cos \left(\omega_{n} t\right)
$$

Los coeficientes $S_{K n}$ y las frecuencias $\omega_{n}$ dependen del momento cuadrupolar Q del núcleo sonda, del valor del espín nuclear $I$ del estado intermedio y de las componentes principales del tensor GCE observado en el sitio de la sonda. Debido a que el núcleo a tiempo $t=0$ aún no ha sido perturbado $\left(G_{K K}(t=0)=1\right)$, entonces los coeficientes $\mathrm{S}_{K n}$ deben satisfacer $\sum_{n=0}^{n_{\text {max }}} S_{K n}=1$. 


\section{2-3 Interacción cuadrupolar eléctrica}

En esta sección vamos a analizar el caso particular de la interacción entre el núcleo y el campo eléctrico del campo cristalino del sólido donde se encuentra alojada la sonda, ya que es la interacción de interés en esta Tesis. Supongamos que el campo magnético del campo extranuclear es lo suficientemente débil como para despreciar la interacción magnética frente a la interacción eléctrica. Entonces, la principal interacción entre el núcleo de carga $Z e$ y el campo cristalino será de tipo Coulombiana, cuantitativamente dada por $\operatorname{ZeV}(0)$ (donde se supone que el núcleo se sitúa en el origen). Debido a que la distribución de carga nuclear no es esférica y teniendo en cuenta que el campo eléctrico cristalino no es homogéneo aparecerán términos correctivos en la energía, rompiéndose así la degeneración y produciendo el desdoblamiento de los subestados $m$ del nivel intermedio $I$. La energía electrostática del sistema debido a la interacción entre el núcleo y el potencial $V_{\text {ext }}$ del campo cristalino es la convolución de la densidad de carga nuclear con dicho potencial:

$$
E_{\text {elec }}=\int \rho_{n}(\vec{r}) V_{\text {ext }}(\vec{r}) d^{3} r
$$

El potencial externo $V_{\text {ext }}(\boldsymbol{r})$ puede desarrollarse en serie de Taylor [Jackson 1964] de modo que la ecuación anterior queda expresada en una serie donde cada término de orden $n$ se relaciona con la multipolaridad de la interacción:

$$
E_{\text {elec }}=q V(0)-\vec{p} \cdot \vec{E}+\frac{1}{6} \sum_{i} \sum_{j} Q_{i j} \frac{\partial}{\partial} \frac{E_{j}(0)}{x_{i}}-\frac{3}{2} \pi \int \rho_{e l}(0) \rho_{n}(\vec{r}) r^{2} d \vec{r}
$$

donde $q$ es la carga total del núcleo y representa el momento monopolar, $\vec{p}$ es el momento dipolar eléctrico, $Q_{i j}$ es el momento cuadrupolar eléctrico nuclear de traza nula, y $\rho_{e l}$ y $\rho_{n}$ son las densidades de carga electrónica y nuclear, respectivamente.

En la ecuación (2.6) se puede apreciar que la carga nuclear se acopla con el potencial electrostático; el momento dipolar eléctrico, con el campo eléctrico; el momento cuadrupolar, con el GCE; y el último término es la contribución de los electrones $s$ que tienen una probabilidad no nula de penetrar en la región nuclear. 
Debido a que la simetría de la densidad carga de electrones $s$ es esférica, el término es de orden cero y, al igual que la interacción entre el momento monopolar y el potencial $V(0)$, sólo produce un corrimiento en la energía electrostática de la interacción. La interacción dipolar se anula debido a la simetría de las funciones de onda nucleares. Por lo tanto, el único término que rompe la degeneración del estado intermedio $I$ es el correspondiente a la interacción entre el momento cuadrupolar eléctrico nuclear y el tensor GCE.

Como ya se mencionó anteriormente, la interacción entre el GCE y el momento cuadrupolar $Q_{i j}$ del núcleo en el estado intermedio $I$, semiclásicamente provoca la precesión del espín nuclear entorno a la dirección del eje de cuantificación.

El tensor GCE queda definido a partir de las nueve componentes $V_{i j}=\frac{\partial^{2} V}{\partial x_{i} \partial x_{j}}, i, j=1,2,3 \cdot V(\vec{r})$ es continuo de orden dos, por lo que la matriz real de $3 x 3$ del tensor GCE es simétrica y por ende diagonalizable, reduciéndose a las tres componentes principales $V_{11}, V_{22}$ y $V_{33}$. Estas tres componentes se definen de manera que $\left|V_{33}\right| \geq\left|V_{22}\right| \geq\left|V_{11}\right|$ y debido a que satisfacen la ecuación de Laplace ( $V_{11}+V_{22}+V_{33}=0$, tensor de traza nula), el tensor GCE diagonalizado queda completamente definido por dos de sus elementos diagonales. Es conveniente definir un parámetro adimensional, el parámetro de asimetría $\eta$, que depende de las tres componentes principales:

$$
\eta=\frac{V_{11}-V_{22}}{V_{33}}
$$

De este modo el GCE queda completamente determinado a partir de $\eta$ y de la componente mayor $V_{33}$.

Este parámetro $\eta$ expresa la desviación del tensor GCE respecto de aquel que posee simetría axial $(\eta=0)$. A partir de la definición de los elementos diagonales del tensor y de $\eta$, se tiene $0 \leq \eta \leq 1$.

El hamiltoniano perturbativo que describe la interacción entre el GCE del campo extranuclear y el momento cuadrupolar del núcleo en el estado intermedio I viene dado por [Frauenfelder, 1968]: 


$$
\widehat{H}_{e}=\sum_{K=0}^{\infty} \sum_{m=-K}^{K} \widehat{Q}_{K}^{m} \widehat{V}_{K}^{m *},
$$

donde $\widehat{Q}_{K}^{m}$ es el operador momento cuadrupolar eléctrico nuclear y $\widehat{V}_{K}^{m}$ es el operador tensor gradiente de campo eléctrico del campo extranuclear. Debido a que trabajamos en la base $|K m\rangle$, ambos operadores se expresan en coordenadas esféricas, esto es $\langle\breve{r} \mid K m\rangle=Y_{m}^{K}(\theta, \varphi)$. De esta manera, se puede demostrar [Rentería, 1992] que los autovalores del hamiltoniano perturbativo de interacción cuadrupolar eléctrica $\hat{H}_{e}$, para $I=5 / 2$, toman la forma:

$$
\begin{gathered}
E_{ \pm 5 / 2}=\frac{1}{\sqrt{3}} \hbar \omega_{Q} \alpha \cos \left(\frac{1}{3} \arccos \beta\right) \\
E_{ \pm 3 / 2}=-\frac{1}{\sqrt{3}} \hbar \omega_{Q} \alpha \cos \frac{1}{3}(\pi+\arccos \beta) \\
E_{ \pm 1 / 2}=-\frac{1}{\sqrt{3}} \hbar \omega_{Q} \alpha \cos \frac{1}{3}(\pi-\arccos \beta),
\end{gathered}
$$

donde $\omega_{Q} \equiv \frac{e Q V_{33}}{40 \hbar} \quad$ es la frecuencia cuadrupolar, $\quad \alpha \equiv 4 \sqrt{7\left(3+\eta^{2}\right)} \quad$ y $\beta \equiv 1920 \sqrt{3}\left(1-\eta^{2}\right) / \alpha^{-3}$.

Debido a que los autovalores de $\hat{H}_{e}$ no dependen del signo de $m$, esto es, del signo de la proyección del espín nuclear I en el eje de cuantificación, entonces las frecuencias de interacción $\omega=\frac{E_{m}-E_{m^{\prime}}}{\hbar}$, tampoco dependerán de dicho signo, por lo que solo habrá tres frecuencias de interacción $\omega_{i}$ :

$$
\begin{gathered}
\omega_{1}=\frac{1}{\hbar}\left(E_{ \pm 3 / 2}-E_{ \pm 1 / 2}\right)=\omega_{Q} \alpha \operatorname{sen}\left(\frac{1}{3} \arccos \beta\right) \\
\omega_{2}=\frac{1}{\hbar}\left(E_{ \pm 5 / 2}-E_{ \pm 3 / 2}\right)=\omega_{Q} \alpha \operatorname{sen}\left(\frac{1}{3}(\pi-\arccos \beta)\right) \\
\omega_{3}=\frac{1}{\hbar}\left(E_{ \pm 5 / 2}-E_{ \pm 1 / 2}\right)=\omega_{Q} \alpha \operatorname{sen}\left(\frac{1}{3}(\pi+\arccos \beta)\right)
\end{gathered}
$$


En la Figura 2.4 se grafican los autovalores de energía, en unidades de $\hbar \omega_{Q}$, en función del parámetro de asimetría $\eta$.

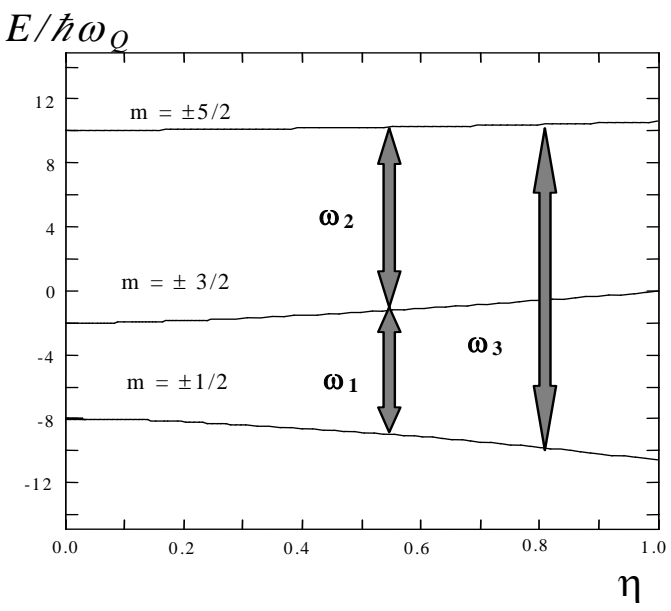

Figura 2.4: Autovalores de energía $\mathrm{E}$ vs. $\eta$. Se identifican las tres frecuencias de interacción.
Observando las energías (2.9) del desdoblamiento del estado intermedio nuclear de espín $I=5 / 2$, vemos que la única información de la interacción cuadrupolar eléctrica necesaria para calcular dichos autovalores reside en $\eta \mathrm{y}$ $\omega_{\mathrm{Q}}$

Las frecuencias de interacción $\omega_{n}$ suelen expresarse como un producto de factores independientes, esto es:

$$
\omega_{n}=g_{n}(\eta) v_{Q}=g_{n}(\eta) \omega_{Q}
$$

con $n=1,2$, 3 .

En la Figura 2.5 se grafican las funciones $g_{n}$, con $n=1$, 2, 3, en función de $\eta$ y los cocientes $\omega_{2} / \omega_{1}$ y $\omega_{3} / \omega_{1}$, y también en función de $\eta$ :
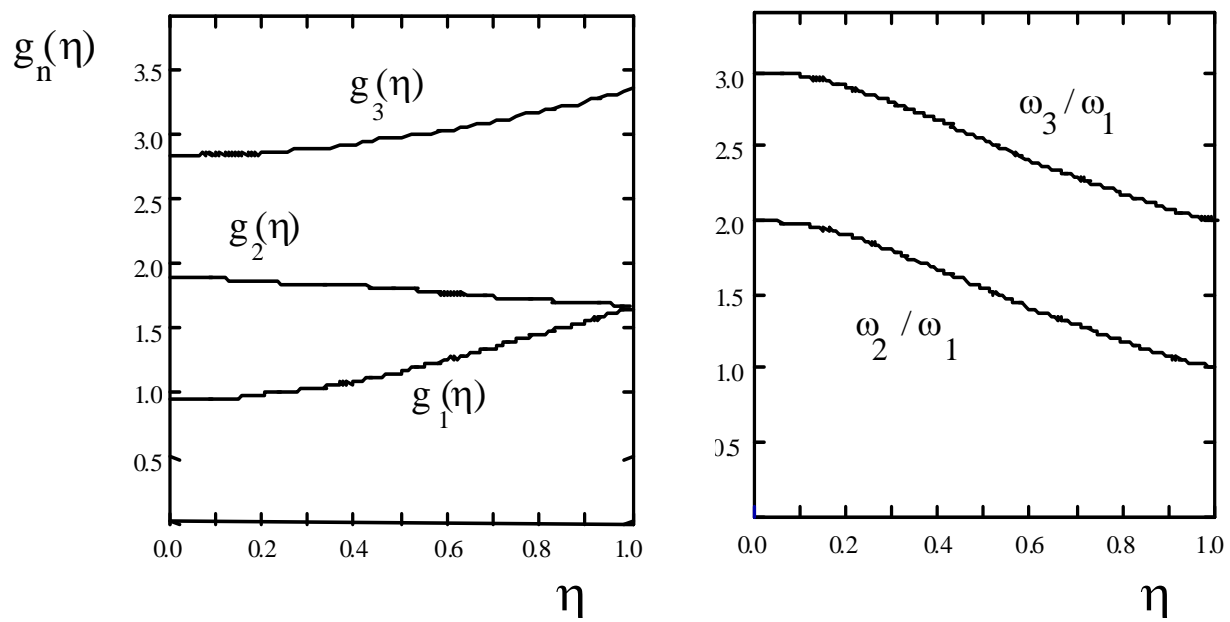

Figura 2.5: Gráfico de $g_{n}(\eta)(n=1,2,3)$ vs. $\eta$ y de los cocientes $\omega_{2} / \omega_{1}$ y $\omega_{3} / \omega_{1}$ en función de $\eta$. 
A modo de ejemplo veamos que forma tienen los desdoblamientos de energías y las frecuencias de transición para el caso de GCE axialmente simétrico, esto es $\eta=0$. El desdoblamiento de energía entre dos subestados arbitrarios $m$ y $m$ ' es de la forma:

$\Delta E_{m, m^{\prime}}=E_{m}-E_{m^{\prime}}=3 e Q V_{33} \frac{\left(m^{2}-m^{\prime 2}\right)}{40}$,

y explícitamente:

$$
\begin{aligned}
& \Delta E_{ \pm 3 / 2, \pm 1 / 2}=6 \omega_{Q} \hbar=\hbar \omega_{1} \Rightarrow \omega_{1}=6 \omega_{Q} \\
& \Delta E_{ \pm 5 / 2, \pm 3 / 2}=12 \omega_{Q} \hbar=\hbar \omega_{2} \Rightarrow \omega_{2}=12 \omega_{Q}=2 \omega_{1} \\
& \Delta E_{ \pm 5 / 2, \pm 1 / 2}=18 \omega_{Q} \hbar=\hbar \omega_{3} \Rightarrow \omega_{3}=18 \omega_{Q}=3 \omega_{1} .
\end{aligned}
$$

Para las sondas empleadas en este trabajo el coeficiente $A_{44}$ (ver ecuación 2.3) es del orden de $1 \%$ con respecto al coeficiente $A_{22}$. Por lo que la función de correlación toma la forma:

$$
W(\theta)=1+A_{22} G_{22}(t) P_{2}(\cos (\theta)) .
$$

El factor de perturbación $G_{22}(t)$ puede escribirse como:

$$
G_{22}(t)=S_{20}(\eta)+\sum_{n=1}^{3} S_{2 n}(\eta) \cos \left(\omega_{n}\left(\eta, \omega_{Q}\right) t\right)
$$

donde los coeficientes $S_{2 n}$ están normalizados $\left(\sum_{n=0}^{3} S_{2 n}=1\right)$. De (2.16) vemos que $G_{22}(t)$ depende únicamente de $\eta$ y $\omega_{\mathrm{Q}}$, es decir, de los parámetros que caracterizan el tensor GCE. Finalmente, vía una medida experimental de la correlación angular (perturbada) de las emisiones $\gamma_{1}$ y $\gamma_{2}$ en la desexcitación del núcleo de interés podremos determinar $\eta$ 
y $\omega_{\mathrm{Q}}$, y si conocemos $Q$ podremos obtener una medida de la componente principal del tensor GCE, $\mathrm{V}_{33}$, experimentada por el núcleo elegido.

\section{2-4 Método Experimental}

La técnica de las Correlaciones Angulares Perturbadas Diferenciales en Tiempo (PAC) se basa en la determinación de la interacción hiperfina entre un núcleo radiactivo y el campo extranuclear del sistema actuante en el sitio donde se aloja el núcleo a partir de la determinación de la correlación angular perturbada entre las radiaciones $\gamma_{1}$ y $\gamma_{2}$ emitidas por el núcleo radiactivo. Entonces, estos núcleos inmersos en un sólido devienen en sondas adecuadas para estudiar propiedades del sistema huésped. Es por esto que cada uno de estos núcleos suele llamarse núcleo-sonda.

\section{2-4-1 Medida de las Correlaciones Angulares Perturbadas Diferenciales en Tiempo}

Para determinar experimentalmente el tensor GCE, conocido el momento cuadrupolar nuclear $Q$ del núcleo-sonda, es necesario obtener el factor de Perturbación $G_{22}(t)$ presentado en (2.16). Para obtenerlo necesitamos medir el número de coincidencias $\gamma_{1}-\gamma_{2}$ en función del tiempo $t$ transcurrido entre ambas emisiones, detectadas en dos posiciones angulares diferentes. Si llamamos $C^{\exp }(\theta, t)$ a las coincidencias medidas para un ángulo $\theta$ entre las radiaciones emitidas y luego de haber transcurrido un tiempo $t$ entre dichas radiaciones, se tiene:

$C^{\mathrm{exp}}(\theta, t)=A P_{1} P_{2} \varepsilon_{1} \varepsilon_{2} \varepsilon_{C} W^{\mathrm{exp}}(\theta, t) e^{-t / \lambda}+C^{A}$

donde $A$ es la actividad de la muestra, $P_{1}$ es la probabilidad de emisión de $\gamma_{1}$ por cada desintegración del núcleo sonda, $P_{2}$ es la probabilidad de emisión de $\gamma_{2}$ habiéndose emitido $\gamma_{1}, \varepsilon_{1} \mathrm{y} \varepsilon_{2}$ son las eficiencias absolutas de los cristales para detectar $\gamma_{1} \mathrm{y} \gamma_{2}$, respectivamente, $\varepsilon_{c}$ es la eficiencia del circuito de coincidencias y $\lambda$ es la vida media del estado intermedio de la cascada $\gamma-\gamma$. Las coincidencias accidentales $C^{A}$ son aquellas en las que $\gamma_{1}$ y $\gamma_{2}$ provienen de diferentes núcleos. Dichas coincidencias son proporcionales al cuadrado de la actividad de la muestra y a la resolución temporal del equipo. En la Figura 2.6 se muestran a modo de ejemplo, espectros de coincidencias típicos. Las cuentas a la izquierda del máximo son las coincidencias accidentales, las que se deben descontar para determinar las coincidencias reales. 


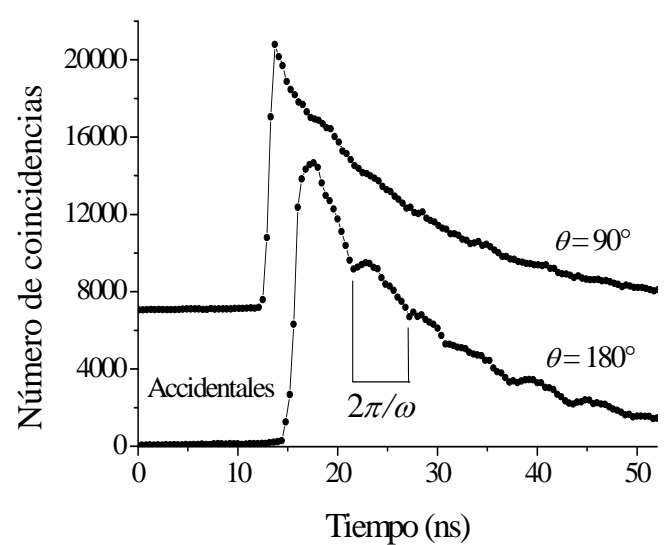

Figura 2.6: Espectros de coincidencias $\gamma_{1}-\gamma_{2}$ para $\theta=90^{\circ}$ y $\theta=180^{\circ}$. Los espectros corresponden a ${ }^{181} \mathrm{Ta}$ en $\mathrm{C}$ $\mathrm{Ho}_{2} \mathrm{O}_{3}$ [Errico 2002].

La dependencia temporal de la función de correlación experimental $W^{\exp }(\theta, t)$ involucra tiempos de emisión anteriores y posteriores a t, debido a que la resolución temporal del equipo experimental $\left(r\left(t-t^{\prime}\right)\right)$ impide medir la correlación exactactamente en $t$. Por lo que dos eventos no simultáneos pueden interpretarse como tales, o bien dos sucesos consecutivos producidos con un retardo dado son detectados con un retardo diferente. Entonces, la función $W^{\exp }(\theta, t)$ se puede describir a partir de la convolución temporal de la función de correlación perturbada teórica $W(\theta, t)$ (ver la expresión (2.15)) con la resolución temporal finita $r\left(t-t^{\prime}\right)$ del equipo experimental y el decaimiento exponencial $e^{-t / \lambda}$ del estado intermedio nuclear $I$ :

$W^{\exp }(\theta, t)=\frac{\int_{0}^{\infty} r\left(t-t^{\prime}\right) W\left(\theta, t^{\prime}\right) e^{-t^{\prime} / \lambda} d t^{\prime}}{\int r\left(t-t^{\prime}\right) e^{-t^{\prime} / \lambda} d t^{\prime}}$

A partir de la expresión (1.14) para la función de correlación obtenemos:

$W^{\exp }(\theta, t)=1+A_{22}^{\exp } G_{22}^{\exp }(t) P_{2}(\cos (\theta))$,

donde hemos definido el factor de perturbación experimental $G_{22}{ }^{\text {exp }}(t)$ como:

$G_{22}^{\exp }(t)=\frac{\int_{0}^{\infty} r\left(t-t^{\prime}\right) G_{22}\left(t^{\prime}\right) e^{-t^{\prime} / \lambda} d t^{\prime}}{\int r\left(t-t^{\prime}\right) e^{-t^{\prime} / \lambda} d t^{\prime}}$. 
La resolución $r\left(t-t^{\prime}\right)$ puede determinarse experimentalmente midiendo la respuesta temporal del equipo ante una excitación del tipo “delta”. En la práctica esto se puede realizar, por ejemplo, detectando la coincidencia de los rayos $\gamma$ de $1100 \mathrm{keV}$ y $1300 \mathrm{keV}$ de la cascada $\gamma-\gamma$ del ${ }^{60} \mathrm{Co}$, donde la vida media del estado intermedio es de $0.73 \mathrm{ps}$, la cual resulta despreciable frente a la resolución típica de un equipo PAC ( $\left.10^{-1} \mathrm{~ns}\right)$. Un método alternativo es medir las dos radiaciones $\gamma$ de $511 \mathrm{keV}$ emitidas en la aniquilación positrón-electrón posterior al decaimiento $\beta^{+}$del ${ }^{22} \mathrm{Na}$. Debido a la conservación del momento y la energía, son emitidos en forma simultánea dos fotones de $511 \mathrm{keV}$ en direcciones opuestas con una diferencia temporal nula [Leo, 1994].

A modo de ejemplo, en la Figura 2.7 se muestra el espectro de coincidencias de la cascada $\gamma-\gamma$ del ${ }^{60}$ Co medido en el espectrómetro PACar de La Plata [Rentería 2008] con las ventanas de energía de la cascada $\gamma-\gamma$ de la sonda $\left({ }^{181} \mathrm{Hf} \rightarrow\right)^{181} \mathrm{Ta}$, el tiempo de resolución del equipo $2 \tau_{\text {res }}$ es el ancho a mitad de altura.

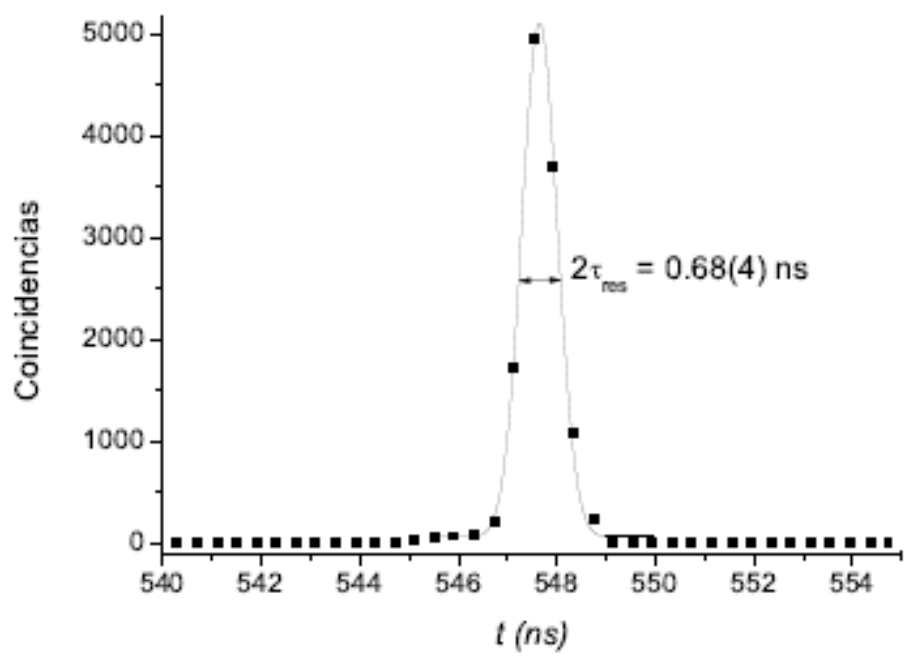

Figura 2.7: Espectro de coincidencias de la cascada 1100 - $1300 \mathrm{keV}$ del ${ }^{60} \mathrm{Co} \mathrm{y}$ resolución temporal del equipo PAC [Rentería, 2008].

De la expresión (2.17) puede apreciarse que para cada tiempo $t$ puede obtenerse un sistema de dos ecuaciones con dos incógnitas $\left(A_{22}{ }^{\exp }\right.$ y $\left.G_{22}{ }^{\text {exp }}\right)$, de modo que midiendo las coincidencias en dos posiciones diferentes de los detectores se puede determinar $G_{22}{ }^{\text {exp }}(t)$. Comúnmente se eligen las posiciones $\theta=90^{\circ}$ y $180^{\circ}$ puesto que la distribución angular de la radiación del núcleo-sonda presenta la mayor diferencia relativa de 
intensidad en tales ángulos. Se define la función $R(t)$, llamada espectro de precesión de espín, relación de asimetría o simplemente espectro PAC, como:

$R(t)=2 \frac{C\left(180^{\circ}, t\right)-C\left(90^{\circ}, t\right)}{C\left(180^{\circ}, t\right)+2 C\left(90^{\circ}, t\right)}$,

donde $C(\theta, t)=C^{\exp }(\theta, t)-C^{A}$.

Reemplazando por la forma explícita de $W^{\text {exp }}(\theta, t)$ (ec. 2.19) obtenemos:

$$
R(t)=2 \frac{1+A_{22}^{\exp } G_{22}^{\exp }(t) P_{2}(-1)-\left(1+A_{22}^{\exp } G_{22}^{\exp }(t) P_{2}(0)\right)}{1+A_{22}^{\exp } G_{22}^{\exp }(t) P_{2}(-1)+2\left(1+A_{22}^{\exp } G_{22}^{\exp }(t) P_{2}(0)\right)}
$$

A partir de la expresión del polinomio de Legendre de orden $2\left(P_{2}(x)=\frac{1}{2}\left(3 x^{2}-1\right)\right)$, tenemos que $P_{2}(-1)=1$ y $P_{2}(0)=-1 / 2$, entonces:

$$
R(t)=A_{22}^{\exp } G_{22}^{\exp }(t)=A_{22}^{\exp } \sum_{n=0}^{3} S_{2 n}(\eta) \cos \left(\omega_{n} t\right),
$$

donde $\omega_{0}=0$.

El espectro PAC definido según (2.21) nos permite obtener $G_{22}{ }^{\text {exp }}(t)$, siendo conocido $\mathrm{A}_{22}$. Cabe destacar que la forma del espectro $\mathrm{R}(\mathrm{t})$ es válida para arreglos experimentales de dos, tres o cuatro detectores.

En la Figura 2.8 se simulan distintos espectros $\mathrm{R}(\mathrm{t})$ para $\mathrm{I}=5 / 2$ y distintos valores de $\eta$, con sus respectivas transformadas de Fourier donde se pueden apreciar las frecuencias de interacción. Puede observarse que la anisotropía puede determinarse midiendo la correlación angular para GCE nulo en el sitio del núcleo sonda (Figura 2.8 (a) izquierda) o del espectro $\mathrm{R}(\mathrm{t})$ a $\mathrm{t}=0$. 

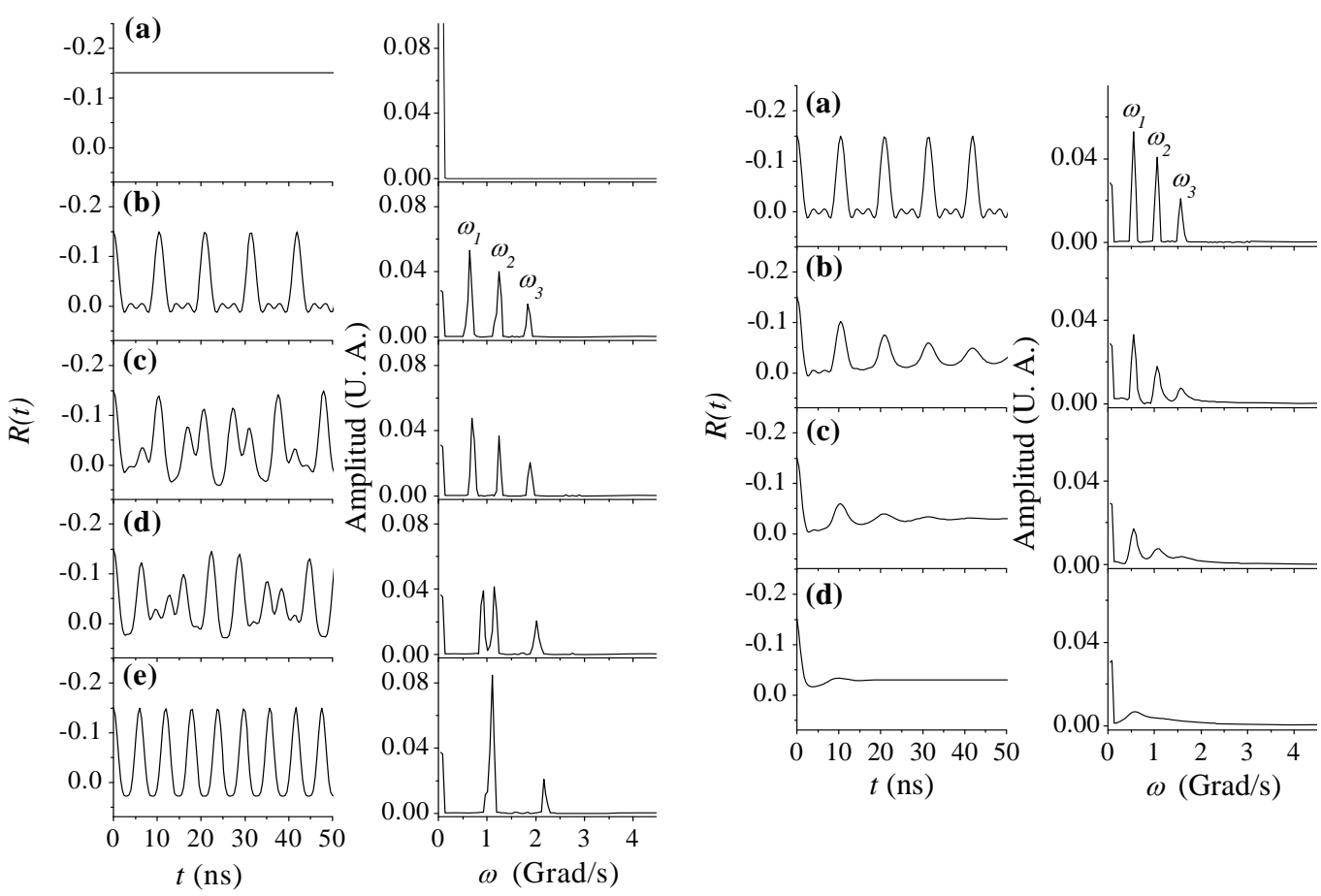

Figura 2.8: Espectros $\mathrm{R}(\mathrm{t})$ simulados para una única interacción caracterizada por: Izquierda: (a) GCE nulo; (b) $\eta=0$; (c) $\eta=0.3$; (d) $\eta=0.7$; (e) $\eta=1$. Para los casos (b)-(e) se consideró $\omega_{Q}=100 \mathrm{Mrad} / \mathrm{s}$. Derecha: (a) $\delta=0$; (b) $\delta$ $=0.05$; (c) $\delta=0.10$; (d) $\delta=0.15$. En todos los casos (menos izquierda (a)) se consideró $\omega_{\mathrm{Q}}=100 \mathrm{Mrad} / \mathrm{s}$ y $\eta=0$.

Resumiendo lo que hemos deducido hasta aquí, hemos encontrado una expresión cuantitativa que da cuenta de la interacción cuadrupolar eléctrica entre el tensor GCE del campo cristalino del sólido donde se aloja la sonda y el momento cuadrupolar nuclear del núcleo-sonda en su estado intermedio de espín I. De esta forma, encontramos una expresión de naturaleza experimental que nos permite ajustar los espectros de rotación de espín experimentales y así obtener el tensor GCE en el sitio de la sonda. Ahora, esta expresión experimental fue hallada a partir de varias hipótesis que es conveniente detallar: la muestra y los detectores se han considerado puntuales, hemos considerado que el sólido bajo estudio constituye una red cristalina perfecta y que las sondas ocupan solamente sitios cristalográficos (propios de dicha red) libres de defectos. Debido a estas idealizaciones es necesario introducir en el factor de perturbación determinadas aproximaciones que nos acerquen de una manera más precisa al factor real:

- Como dijimos anteriormente, para el cálculo de la función de correlación angular $W(\theta, t)$ hemos supuesto puntuales los cristales centelladores de los detectores y la fuente radiactiva. Su tamaño finito y por ende la abertura de ángulo sólido no 
infinitesimal subtendida desde la muestra hasta los detectores provoca la atenuación de la anisotropía $A_{22}$, por lo que $W^{e x p}(\theta, t)$ tendrá una amplitud menor que la teórica. Para reducir estas anomalías se utilizan fuentes lo más puntuales posible y se trata de reducir la abertura del ángulo sólido aumentando la distancia muestra-detector, pero teniendo en cuenta que el número de eventos detectados decrece con el cuadrado de dicha distancia.

- Un cristal real no puede considerarse como una red cristalina perfecta sin imperfecciones como ser impurezas, dislocaciones de planos, defectos puntuales como vacancias, átomos en los intersticios, daños por la radiación, etc. Estas imperfecciones producen una distribución finita $\delta$ en las frecuencias cuadrupolares $\omega_{n}$ alrededor de sus valores medios. Dicha distribución puede suponerse una distribución gaussiana o lorentziana $P\left(\omega_{n}\right)$. En la Figura 2.8 (derecha) se aprecia como estas distribuciones atenúan las oscilaciones en el espectro $R(t)$. Por lo tanto, el factor de perturbación teórico debe ser pesado con las distribuciones $P\left(\omega_{n}\right)$ en la forma:

$$
G_{22}(t) \rightarrow \int_{-\infty}^{\infty} P(\omega) G_{22}(t) d \omega
$$

- Las sondas pueden ubicarse en sitios con entornos cristalográficos distintos, esto es, en sitios no equivalentes, aún para el caso de una red cristalina perfecta. Entonces, el factor de perturbación $G_{22}(t)$ más general que podamos tener será una combinación lineal de la forma: $G_{22}(t)=f_{0}+\sum_{i} f_{i} G_{22}^{i}(t)$, donde $f_{i}$ es la fracción de núcleos sonda que experimentan una interacción hiperfina caracterizada por los parámetros $\omega_{\mathrm{Q} i} \mathrm{y} \eta_{i}$ en el sitio inequivalente $i$ y $f_{0}$ es la fracción de núcleos sonda que experimenta un GCE nulo. Dichas fracciones deben cumplir $f_{0}+\sum_{i} f_{i}=1$

- Suponiendo que la respuesta temporal del equipo experimental (ver Figura 2.7) es gaussiana, y teniendo en cuenta que una función gaussiana y su transformada de Fourier tienen el mismo aspecto funcional, por lo que la convolución de $W(\theta, t)$ con dicha respuesta nos da $W^{\text {exp }}(\theta, t)$ multiplicado por tal distribución. Esto nos lleva a introducir factores reales del tipo:

$$
S_{2 n}^{\text {real }}=S_{2 n}(\eta) \cdot e^{-\frac{1}{2} \tau_{\text {res }}^{2} \omega_{n}^{2}}
$$


Como ejemplo, en la Figura 2.9 se muestra como varía el espectro $R(t)$ con la resolución temporal, a través de dos espectros $\mathrm{R}(\mathrm{t})$ para la misma muestra, $\mathrm{K}_{2} \mathrm{ZrF}_{6}$
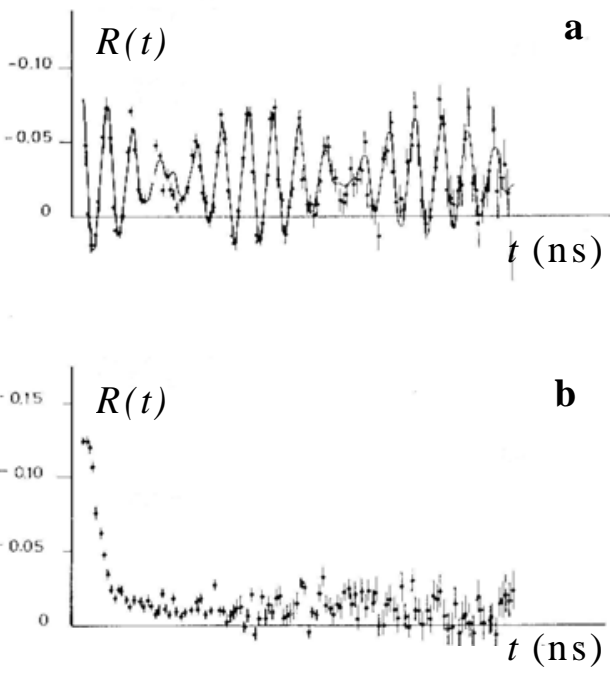

Figura 2.9: Espectros $\mathrm{R}(\mathrm{t})$ de ${ }^{181} \mathrm{Ta}$ en $\mathrm{K}_{2} \mathrm{ZrF}_{6}$ tomados con equipos con diferente resolución temporal: a) $\operatorname{CsF}(\sim 0,8 \mathrm{~ns})$; b) $\mathrm{NaI}(\mathrm{Tl})$ ( $3 \mathrm{~ns})$ [Martinez, 1981]. dopado con ${ }^{181} \mathrm{Ta}$, medida con dos detectores de resolución temporal muy diferente. Claramente puede apreciarse que las oscilaciones del espectro $R(t)$ tienden a desaparecer cuanto menor sea la resolución temporal.

Por lo tanto, considerando todas las modificaciones introducidas tendientes a acercar la expresión del $R(t)$ a su forma real, dicha expresión queda:

$$
R(t)=A_{22}^{\exp }\left(f_{0}+\sum_{i} f_{i} G_{22}^{i}(t)\right) .
$$

El factor de perturbación para cada sitio inequivalente $i$ tiene la forma:

$$
G_{22}^{i}(t)=\sum_{n=0}^{3} S_{2 n}^{i}\left(\eta^{i}\right) \cos \left(\omega_{n}^{i} t\right) e^{-\left(\omega_{n}^{i} \delta_{Q}^{i} t\right)^{p} / p}
$$

El factor exponencial proviene de convolucionar $G_{22}(t)$ con la distribución de frecuencias $P(\omega)$. Si dicha distribución es lorentziana $(p=1), P(\omega)$ toma la forma:

$$
P(\omega)=\frac{\delta}{\pi\left(\delta^{2}+\frac{(\langle\omega\rangle-\omega)^{2}}{\omega^{2}}\right)},
$$

y si es gaussiana $(p=2)$, se tiene:

$$
P(\omega)=\frac{1}{\delta \sqrt{2 \cdot \pi}} \cdot e^{-(\langle\omega\rangle-\omega)^{2} / 2 \delta^{2}}
$$




\section{2-4-2 Equipo experimental}

La determinación experimental de $G_{22}(t)$ da como resultado la determinación de los parámetros hiperfinos característicos de la interacción cuadrupolar eléctrica entre el momento cuadrupolar Q del núcleo-sonda y el tensor GCE del campo extranuclear del sólido. Para esto es necesario medir las coincidencias $C(\theta, t)$ de la cascada $\gamma-\gamma$ del núcleo para dos ángulos distintos. Es decir, medir $\gamma_{1}$ en el detector 1 en coincidencia con la detección de $\gamma_{2}$ en el detector 2 . Tal medición está caracterizada por la energía de ambas radiaciones y por la determinación precisa de la diferencia temporal entre la emisión de $\gamma_{1} \mathrm{y} \gamma_{2}$

La mayor parte de los experimentos PAC cuyos resultados se describen en el Capítulo 5 fueron desarrollados durante mi estadía (Julio - Agosto de 2009) en el Instituto de Pesquisas Energéticas y Nucleares (IPEN) de la Universidad de San Pablo utilizando el espectrómetro PAC de cuatro detectores perteneciente al grupo de Interacciones Hiperfinas del IPEN. El primero de los experimentos en $\mathrm{SnO}$ fue realizado con el mismo espectrómetro 2 años antes. El exhaustivo análisis de sus espectros y el desarrollo preliminar del modelo que describe estos resultados a partir de cálculos de primeros principios precedió y preparó el desarrollo del resto de los experimentos y la elección de los sistemas estudiados. Medidas preliminares de óxidos binarios dopados con la sonda $\left({ }^{111} \mathrm{In} \rightarrow\right)^{111} \mathrm{Cd}$ se realizaron en el equipo PACar de lógica rápida-rápida [Rentería 2008] (optimizado para ser utilizado con la sonda $\left.\left({ }^{181} \mathrm{Hf} \rightarrow\right)^{181} \mathrm{Ta}\right)$, pero la aparición de oscilaciones espurias en el equipo para las energías de la cascada $\gamma-\gamma$ del ${ }^{111} \mathrm{Cd}$ (ver Fig. 2.10) imposibilitaba una correcta determinación de los espectros. En consecuencia, decidimos utilizar un equipo de lógica rápida-lenta y de mayor eficiencia, óptimo para ser utilizado con el isótopo ${ }^{111} \operatorname{In}\left(\rightarrow{ }^{111} \mathrm{Cd}\right)$, el cual posee una vida media mucho menor que el ${ }^{181} \mathrm{Hf}\left(\rightarrow{ }^{181} \mathrm{Ta}\right)$. Finalmente, el análisis de todos los espectros PAC fue realizado en La Plata.

Los detectores están compuestos por cristales centelladores de Floruro de Bario $\left(\mathrm{BaF}_{2}\right)$. La forma de los cristales del tipo cono truncado (ver. Fig. 2.9). La forma cónica de los centelladores mejora la respuesta temporal de los detectores minimizando el tiempo de colección de luz dentro del cristal centellador [Butz, 1989]. Cada centellador está acoplado ópticamente a un fotomultiplicador. La señal de cada detector es procesada de dos maneras diferentes independientes entre sí, en un circuito de electrónica rápida-lenta como se muestra en la Figura 2.9. Por cada radiación gamma 
detectada se producen dos pulsos de tensión, uno proveniente de la salida de dinodo del fotomuliplicador (rama lenta) cuya altura del pulso es proporcional a la energía del fotón; y el otro el otro proveniente de la salida de ánodo (rama rápida), saturado en altura y de rápido crecimiento inicial, por lo que se lo utiliza para indicar el momento de detección de la radiación.

En la rama lenta, los pulsos son conformados por el Pre-Amplificador (PA) y amplificados por el Amplificador (AMP). Un analizador Mono-Canal (SCA) selecciona las energías deseadas en cada detector, produciendo pulsos lógicos que alimentan el Circuito de Coincidencias Lento representado por la Unidad de Direccionamiento (Rounting) [Domienikan 2001]. Observar que los SCA están definidos con un número $i$ que depende del detector al cual se encuentra conectado (1, 2, 3 o 4), y también se definen con una letra $a$ o $b$ que esquematiza si se selecciona $\gamma_{1}(a)$ o $\gamma_{2}(b)$.

En la rama rápida, sólo se analiza el tiempo entre los dos pulsos, independientemente de su energía. A partir de un Discriminador a Fracción Constante (CFD) se eliminan las señales espureas de los pulsos de ánodo y por medio de sus tiempos de crecimiento se determina el instante en que han llegado al detector, generando un pulso lógico. Dado un par de detectores que intervendrá en una coincidencia, uno de ellos provee el pulso lógico de comienzo de la coincidencia (start) y el otro el de finalización de la misma (stop). Estos pulsos alimentan al Convertidor de Tiempo en Amplitud (TAC), el cual genera un pulso de altura proporcional a la diferencia de tiempo de llegada de los pulsos start y stop.

El espectrómetro utilizado es capaz de analizar los doce posibles espectros de coincidencias que se pueden obtener con un arreglo coplanar de cuatro detectores. Para un mismo detector, la salida de cada SCA (rama lenta) está conectado con la salida del contador CFD (rama rápida) del detector a una compuerta AND. Cada salida de ánodo (rama rápida) de los detectores presenta un retardo (D) para producir el retraso adecuado para que la señal de la rama rápida y la de la rama lenta lleguen correlacionadas a la compuerta AND. De este modo se tienen ocho compuertas AND debido a los cuatro detectores y a las dos radiaciones $\gamma_{1}$ y $\gamma_{2}$ detectadas en cada uno. Las salidas de las compuertas AND correspondientes al $\gamma_{1}$ se conectan a una compuerta OR (OR start) y las cuatro salidas AND del $\gamma_{2}$ se conectan a otra compuerta OR (OR stop). La salida de cada compuerta OR se encuentra conectada a un retardo (D) que tiene como función correr el cero de tiempos y permitir detectar las coincidencias 
accidentales en una región pequeña de canales a la izquierda del cero de las coincidencias verdaderas (“tiempos negativos”, correspondientes a algún $\gamma_{2}$ proveniente de un núcleo diferente al que emitió el $\gamma_{1}$ y que llega antes que $\gamma_{1}$ ) para poder restarlas tal como vimos en la sección 2-4-1.

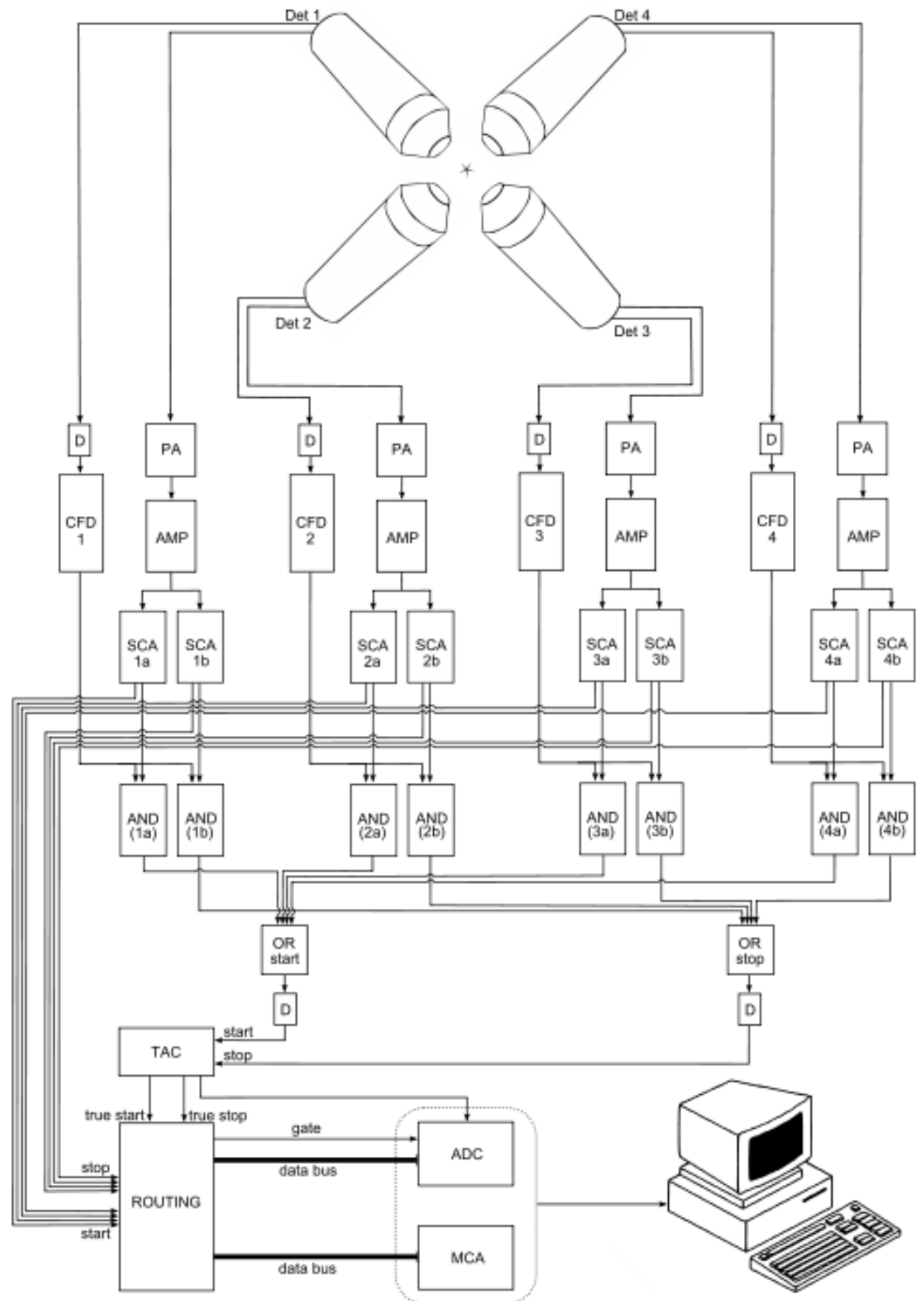

Figura 2.9: Esquema del equipo PAC de cuatro detectores utilizado para medir los sistemas estudiados. 
Las salidas start (stop) de la compuerta OR start (OR stop) son direccionadas al TAC. Por cada pulso start (stop) que arriba al TAC, este produce un pulso de validación true start (true stop) que es enviado al Routing para validar los pulsos que llegan a este desde la rama lenta. El routing entrega un pulso de compuerta (gate) cada vez que se produce una coincidencia de las cuatro posibles. La salida del TAC posee un retardo variable para garantizar que el gate esté presente cuando los pulsos lógicos del TAC lleguen al Convertidor Analógico-Digital (ADC) para luego ser digitalizados. Los pulsos digitalizados por el ADC en presencia del gate son enviados a la memoria correspondiente de las doce existentes del Analizador Multicanal (MCA) según la combinación (de las doce posibles) que ha detectado el Routing.

\section{2-4-3 La sonda PAC $\left({ }^{111} \mathrm{In}(\mathrm{EC}) \rightarrow\right){ }^{111} \mathrm{Cd}$}

Las sondas radiactivas más utilizadas en espectroscopía PAC son $\left({ }^{181} \mathrm{Hf} \rightarrow\right)^{181} \mathrm{Ta}$ y $\left({ }^{111} \mathrm{In} \rightarrow\right){ }^{111} \mathrm{Cd}$. Ambas tienen el espín del estado intermedio $I=5 / 2$ y presentan características compatibles para realizar medidas PAC en cualquier tipo de sistema, las cuales se pueden resumir según:

- Parámetros nucleares conocidos.

- Adecuada anisotropía para la cascada $\gamma-\gamma$ donde se desea realizar la medida PAC.

- Energía de las radiaciones $\gamma$ elevadas de modo de evitar la absorción en el material donde se aloja la sonda o donde se sostiene la muestra.

- La energía entre $\gamma_{1}$ y $\gamma_{2}$ es mayor que la resolución en energía de los detectores utilizados.

- Momento cuadrupolar nuclear $Q$ alto de manera que la frecuencia $\omega_{Q}$ sea observable en el rango de tiempos dado por la vida media del estado intermedio.

- Vida media $\lambda$ del estado intermedio acotado por $T=2 \pi / \omega_{Q}$ de manera tal que $T<\lambda$ para poder determinar con precisión $\omega_{\mathrm{Q}}$.

- Frecuencias $\omega$ limitadas por $\lambda$ y la resolución temporal $\tau_{\text {res }}$ de manera que pueda medirse $\omega$ si cumple $2 \pi / 5 \lambda<\omega<2 \pi / \tau_{\text {res }}$. El límite superior está dado por $\tau_{\text {res }} \mathrm{y}$ el inferior por $5 \lambda$ (suponiendo que es el máximo tiempo que puede medirse estadísticamente). 
En la Tabla 2.1 mostramos los parámetros nucleares de la sonda $\left({ }^{111} \mathrm{In} \rightarrow\right){ }^{111} \mathrm{Cd}$ que es la utilizada en el presente trabajo, como así también los parámetros de la sonda $\left({ }^{111 \mathrm{~m}} \mathrm{Cd} \rightarrow\right)^{111} \mathrm{Cd}$ debido que es el caso de la misma sonda producida desde diferente padre radiactivo, esto tiene incidencia directa en los sistemas a estudiar como veremos más adelante. En la Figura 2.10 mostramos el esquema de decaimiento del $\left({ }^{111} \mathrm{In} \rightarrow\right)^{111} \mathrm{Cd}$ y del $\left({ }^{111 \mathrm{~m}} \mathrm{Cd} \rightarrow\right)^{111} \mathrm{Cd}$.

\begin{tabular}{ccccccccc}
\hline \hline $\begin{array}{c}\text { Isótopo } \\
\text { padre }\end{array}$ & $\begin{array}{c}\text { Vida } \\
\text { media }\end{array}$ & $\begin{array}{c}\text { Isótopo } \\
\text { hijo }\end{array}$ & $\gamma_{1}-\gamma_{2}(\mathrm{keV})$ & $I$ & $Q[b]^{(\mathrm{a})}$ & $\lambda(\mathrm{ns})$ & $A_{22}(\%)^{(\mathrm{b})}$ & $A_{44}(\%)^{(\mathrm{c})}$ \\
\hline${ }^{111} \mathrm{In}$ & $3.6 \mathrm{~d}$ & ${ }^{111} \mathrm{Cd}$ & $171-245$ & $5 / 2$ & $0.83_{13}$ & 84.1 & $-18.0_{1}$ & $0.2_{3}$ \\
\hline${ }^{111 \mathrm{~m}} \mathrm{Cd}$ & $48.6 \mathrm{~m}$ & ${ }^{111} \mathrm{Cd}$ & $151-245$ & $5 / 2$ & $0.83_{13}$ & 84.1 & $17.5_{1}$ & $-0.4_{3}$ \\
\hline \hline
\end{tabular}

Tabla 2.1: Parámetros nucleares de las sondas $\left({ }^{111 \mathrm{~m}} \mathrm{Cd} \rightarrow\right){ }^{111} \mathrm{Cd}$ y $\left({ }^{111} \mathrm{In} \rightarrow\right)^{111} \mathrm{Cd}$.

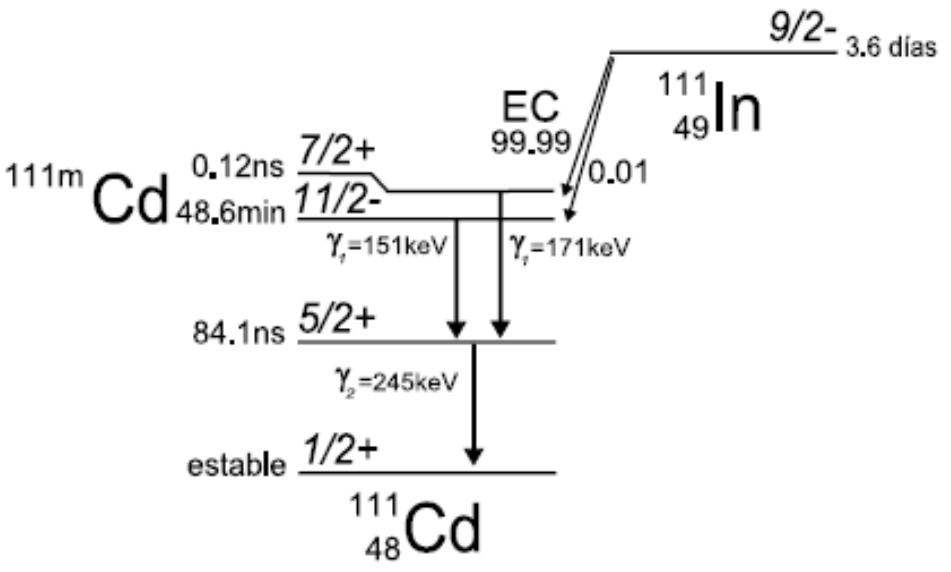

Figura 2.10: Esquemas de decaimiento de la sonda $\left({ }^{111} \mathrm{In} \rightarrow\right)^{111} \mathrm{Cd}$ y de la sonda $\left({ }^{111 \mathrm{~m}} \mathrm{Cd} \rightarrow\right)^{111} \mathrm{Cd}$.

\footnotetext{
(a) [Herzog 1980].

(b) Para ambas sondas [Butz 1983].

(c) Para ambas sondas [Segeth 1987].
} 


\section{Referencias}

[Jackson, 1964] J. D. Jackson, “Classical Electrodynamics”, John Wiley \& Sons, New York (1964).

[Frauenfelder, 1968] H. Frauenfelder y R. M. Steffen en “Alpha-Beta-and Gamma-Ray Spectoscopy”, vol.2 (North- Holland, Amsterdam 1968) P.997.

[Rentería, 1992] M. Rentería, Tesis Doctoral, cap.2, Biblioteca del Departamento de Física, Facultad de Ciencias Exactas, Universidad Nacional de La Plata (1992).

[Errico 2002] L.A. Errico Tesis Doctoral, cap.2, Biblioteca del Departamento de Física, Facultad de Ciencias Exactas, Universidad Nacional de La Plata (2002).

[Leo, 1994] W. R. Leo, “Techniques for Nuclear and Particle Physics Experiments”, $2^{\circ}$ edición, Springer Verlag, New York (1994).

[Rentería 2008] M. Rentería, A.G. Bibiloni, G.N. Darriba, L.A. Errico, E.L. Muñoz, D. Richard y J.Runco, Hyp. Int. 181, 145 (2008).

[Martinez 1981] J.A. Martinez, A.M. Rodriguez, M.C. Caracoche, R.C. Mercader y A. Lopez García, Hyp. Int. 11, 261 (1981).

[Butz, 1989] T. Butz, S. Saibene, Th. Fraenzke y M. Weber, Nuc. Instr. and Meth. A 284, 417 (1989).

[Domienikan 2001] C. Domienikan M. Sc. Thesis, IPEN, San Pablo (2001).

[Herzog 1980] P. Herzog, K. Freitag, M. Reuschenbach y H. Walitzki, Z. Phys. A 294, 13 (1980).

[Butz, 1983] T. Butz y A. Lerf, Phys. Lett. A. 97, 217, (1983).

[Segeth 1987] W. Segeth, Tesis Doctoral, Rijksuniversiteit Groningen, Holanda (1987). 


\section{Capítulo $\mathbf{N}^{0} 3$}

\section{Interacciones hiperfinas dinámicas}

\section{3-1 Comentarios sobre interacciones hiperfinas dinámicas}

Como vimos anteriormente, para caracterizar la interacción hiperfina entre el momento cuadrupolar del estado intermedio de la cascada $\gamma-\gamma$ de un núcleo-sonda y el tensor GCE del campo extranuclear, es necesario medir la perturbación de la correlación angular entre las emisiones de dichas radiaciones, por ejemplo, a partir de la técnica PAC. Un amortiguamiento estático del espectro $R(t)$ tendrá lugar si diferentes núcleos experimentan diferentes GCEs independientes del tiempo (ver Capítulo 2). En cambio, tendremos una situación dinámica cuando el entorno de un dado núcleo cambie durante la vida media del estado intermedio de la cascada $\gamma-\gamma$ dando lugar a un amortiguamiento dinámico debido a que el GCE que “observa” el núcleo-sonda ahora es dependiente del tiempo. Este hecho en el espectro PAC se refleja en una pérdida irreversible de la anisotropía en función del tiempo.

En el caso de la sonda $\left({ }^{111} \mathrm{In} \rightarrow\right)^{111} \mathrm{Cd}$, la captura electrónica (CE) de un electrón de la capa $K$ del In durante el proceso ${ }^{111} \mathrm{In}(\mathrm{EC}) \rightarrow{ }^{111} \mathrm{Cd}$ deja a la sonda ${ }^{111} \mathrm{Cd}$ en un estado cargado positivamente y en una situación energética lejana del equilibrio. El hueco electrónico creado durante la CE es llenado por alguno de los electrones que decaen de capas atómicas más externas en un tiempo que es del orden de $10^{-14} \mathrm{~s}$, por lo que este proceso es demasiado rápido como para afectar el espectro $R(t)$. Sin embargo, durante este proceso el ión pierde algunos electrones (3-8 [Carlson, 1966]) debido a transiciones radiactivas y procesos Auger. En un sólido, electrones que provienen de átomos vecinos próximos a la sonda compensan estos estados cargados en un tiempo del orden de $10^{-12}$ s. Entonces, sondas con uno o dos huecos electrónicos extras $\left(\mathrm{Cd}^{+3} \mathrm{o} \mathrm{Cd}^{+4}\right)$ con respecto al estado iónico de equilibrio en el sólido $\left(\mathrm{Cd}^{+2}\right)$ pueden permanecer con dicha configuración electrónica suficiente tiempo como para alcanzar la ventana temporal del estado intermedio de la cascada $\gamma-\gamma$ del ${ }^{111} \mathrm{Cd}$. Durante esta ventana temporal ocurrirán transiciones al estado más estable, las cuales serán función de las características electrónicas de la red huésped (movilidad y disponibilidad electrónica, por ejemplo). 
Finalmente, estas transiciones pueden modificar la distribución de carga en las proximidades de la sonda, dando lugar a procesos dinámicos que pueden ser monitoreados por la técnica PAC.

\section{3-2 Modelos de "after-effects”}

Existen en la literatura varios modelos que dan cuenta de los AE posteriores a la EC del ${ }^{111} \operatorname{In}\left(\rightarrow{ }^{111} \mathrm{Cd}\right)$. Estos modelos describen el efecto de los after-effects sobre los espectros PAC correspondientes. Asi mismo, en cada modelo se definen parámetros dinámicos que caracterizan la presencia de las interacciones dinámicas en el sistema bajo estudio. En lo que sigue vamos a describir dos modelos de AE: el modelo de Bäverstam y Othaz [Bäverstam, 1972], basado en el modelo pionero de interacciones hiperfinas dinámicas de Abragam y Pound [Abragam, 1953]; y el modelo de Relajación Electrónica Unidireccional de D. Lupascu [Lupascu, 1996].

\section{3-2-1 Modelo de Bäverstam y Othaz}

El proceso dinámico que tiene lugar luego del decaimiento por EC del isótopo ${ }^{111}$ In (after-effects) en el entorno subnanoscópico del núcleo-sonda puede describirse a partir de la estructura de bandas del sólido bajo estudio. Recordemos que el efecto sobre la estructura de bandas del sistema puro al dopar el sólido con una dada impureza es introducir niveles de impureza cercanos a la banda de valencia si la impureza actúa como aceptora o cercanos a la banda de conducción si es donora.

Supongamos que el núcleo de la impureza ${ }^{111}$ In ha capturado un electrón de la capa $\mathrm{K}$ dando comienzo al decaimiento ${ }^{111} \mathrm{In}(\mathrm{EC}) \rightarrow{ }^{111} \mathrm{Cd}$. Entonces, la difusión de un hueco en la banda de valencia, la cual es muy rápida (del orden de $10^{-14} \mathrm{~s}$ ), lleva al átomo sonda altamente ionizado a un estado en el que solo los huecos atrapados en un nivel de impureza cercano a la banda de valencia permanecerán lo suficiente como para modificar la correlación angular. La interacción hiperfina dinámica que se enciende si la EC ocurre, se apaga si el hueco atrapado se recombina con electrones provenientes de la banda de conducción o de valencia por excitación térmica. La probabilidad para el átomo-sonda de alcanzar su estado fundamental entre los tiempos $t$ y $t+d t$ está dado por:

$$
P_{g}(t) d t=\lambda_{g} e^{-\lambda_{g} t} d t
$$


En este modelo se define $\lambda_{\mathrm{g}}$ como la constante de recuperación atómica debido a que al llegar el electrón al nivel la interacción hiperfina deviene en estática y la señal en el espectro PAC se recupera. Su inverso $\tau_{g}=1 / \lambda_{g}$ se define como la vida media del hueco en el nivel de impureza. Por lo tanto, la inversa de la constante de recuperación $\lambda \mathrm{g}$ asociada con la interacción dinámica provee una medida de la vida media del hueco en un centro “atractor” [Bibiloni 1985].

La intensidad media de la interacción dinámica está caracterizada por la constante de relajación de Abragam y Pound $\lambda_{r}$, la cual se supone constante durante el tiempo que los huecos están ligados a la sonda. Mientras el proceso dinámico tiene lugar, la interacción dinámica presente es mucho más intensa que la interacción estática. Bajo estas condiciones el factor de perturbación puede expresarse como el producto de un factor estático, el cual caracteriza la interacción estática resultante, y un factor dinámico que da cuenta del AE experimentado por el núcleo-sonda:

$$
G_{22}(t)=G_{22}^{s}(t) \cdot G_{22}^{d}(t)
$$

donde $G_{22}^{s}(t)$ es el factor de perturbación estático (ver ecuación 2.27) descripto en el Capítulo 2 y $G_{22}^{d}(t)$ es el factor dinámico que describe el AE y que en el marco de este modelo toma la forma(ver Apéndice II):

$$
G_{22}^{d}(t)=\frac{\lambda_{g}}{\lambda_{g}+\lambda_{r}}+\frac{\lambda_{r}}{\lambda_{g}+\lambda_{r}} e^{-\left(\lambda_{g}+\lambda_{r}\right) t}
$$

Si definimos los parámetros $\alpha=\frac{\lambda_{r}}{\lambda_{g}+\lambda_{r}}$ y $\gamma=\lambda_{g}+\lambda_{r}$, la transformación inversa toma la forma $\lambda_{g}=\gamma(1-\alpha)$ y $\lambda_{r}=\alpha \gamma$, entonces el factor de perturbación dinámico reparametrizado en términos de $\alpha$ y $\gamma$ nos queda:

$$
G_{22}^{d}(t)=(1-\alpha)+\alpha e^{-\gamma t}
$$


En este esquema $\alpha$ queda definido como la población de la interacción dinámica, es decir la población de átomos-sondas que a una dada temperatura observan un GCE dependiente del tiempo. Mientras que el parámetro $\gamma$ resulta ser la constante de relajación efectiva de la interacción dinámica. Resulta práctico escribir explícitamente el factor de perturbación con esta nueva parametrización:

$G_{22}(t)=(1-\alpha)\left[\sum_{n=0}^{3} S_{2 n}(\eta) \cos \left(\omega_{n} t\right) e^{-\left(\omega_{n} \delta_{Q} t\right)}\right]+\alpha e^{-\gamma t}\left[\sum_{n=0}^{3} S_{2 n}(\eta) \cos \left(\omega_{n} t\right) e^{-\left(\omega_{n} \delta_{Q} t\right)}\right]$.

Cabe destacar que el GCE caracterizado por $\omega_{\mathrm{n}}$ y $\eta$ en la ecuación (3.5) corresponde al GCE final estable observado por la sonda $\left({ }^{111} \mathrm{In} \rightarrow\right){ }^{111} \mathrm{Cd}$ luego del proceso dinámico. Finalmente, desde el mejor ajuste de las medidas PAC con el factor de perturbación definido en este modelo es posible obtener $\alpha$ y $\gamma$, o unívocamente, $\lambda_{\mathrm{r}}$ y $\lambda_{\mathrm{g}}$ a una temperatura dada.

\section{3-2-2 Modelo de Relajación Electrónica Unidireccional}

En este modelo se supone que el núcleo-sonda posee un conjunto de estados iniciales accesibles, siendo posibles transiciones (dinámicas) entre ellos, cada uno de estos estados está caracterizado por un dado GCE. También se supone que el núcleo sonda tiene un estado final estable caracterizado por un GCE estático. Las transiciones entre los estados iniciales y desde éstos al estado final (o fundamental) están caracterizadas por una única velocidad de relajación (o de transición) $\Gamma_{r}$. La unidireccionalidad del proceso consistente en transiciones desde los estados finales a un único estado final estable es lo que caracteriza al modelo y es análogo al proceso on-off que describe el modelo de Bäverstam and Othaz.

Supongamos un núcleo sonda que experimenta un GCE inicial (frecuencia cuadrupolar inicial $\omega^{i}$ ) y un GCE final (frecuencia cuadrupolar final $\omega^{f}$ ), entonces el factor de perturbación dinámico en este modelo toma la forma:

$$
\begin{aligned}
G_{22}(t)=\sum_{n=0}^{3} & S_{2 n}\left\{\left(1-a_{n}\right) \cos \left(\omega_{n}^{i} t\right) e^{-\left(\omega_{n}^{i} \delta_{Q}^{i}+\Gamma_{r}\right) t}-b_{n} \operatorname{sen}\left(\omega_{n}^{i} t\right) e^{-\left(\omega_{n}^{i} \delta_{Q}^{i}+\Gamma_{r}\right) t}+\right. \\
+ & \left.a_{n} \cos \left(\omega_{n}^{f} t\right) e^{-\omega_{n}^{f} \delta_{Q}^{f} t}+b_{n} \operatorname{sen}\left(\omega_{n}^{f} t\right) e^{-\omega_{n}^{f} \delta_{Q}^{f} t}\right\}
\end{aligned},
$$


donde los coeficientes $a_{n}$ y $b_{n}$ son funciones de la diferencia entre las frecuencias cuadrupolares $\omega_{Q}{ }^{i}$ y $\omega_{Q}{ }^{f}$, de la diferencia de las distribuciones $\delta_{Q}{ }^{i}$ y $\delta_{Q}{ }^{f}$, y de la velocidad de relajación $\Gamma_{r}$. Explícitamente estos coeficientes toman la forma:

$a_{n}=\frac{\Gamma_{r}\left(n \Delta \delta+\Gamma_{r}\right)}{\left(n \Delta \delta+\Gamma_{r}\right)^{2}+(n \Delta \omega)^{2}}, b_{n}=\frac{\Gamma_{r} n \Delta \omega}{\left(n \Delta \delta+\Gamma_{r}\right)^{2}+(n \Delta \omega)^{2}}$,

donde $\Delta \delta=\delta_{Q}{ }^{i}-\delta_{Q}{ }^{f}$ y $\Delta \omega=\omega_{Q}{ }^{i}-\omega_{Q}{ }^{f}$.

Analizando las expresiones (3.5) y (3.6) vemos que si despreciamos los términos con coeficiente $b_{n}$ en (3.6) las expresiones devienen similares con la diferencia que en (3.5) siempre se tiene el GCE final estable observado y en cambio en la ec. (3.6) se definen un GCE inicial y un GCE final (una frecuencia inicial $\omega_{Q}{ }^{i}$ y una frecuencia final $\left.\omega_{Q}^{f}\right)$. En esta comparación el coeficiente (1- $\left.a_{n}\right)$ en (3.6) juega el papel del parámetro $\alpha$ en (3.5), es decir es el coeficiente que cuantifica la población de la interacción dinámica.

En presencia de interacción dinámica, es decir para que el AE sea apreciable el hueco debe permanecer un tiempo suficiente en el nivel de impureza como para llegar a ser “detectado” por la ventana temporal de la medida PAC, este tiempo es del orden de $10^{-1} \mathrm{~ns}$, esto se traduce en una constante de recuperación $\lambda_{g}$ (modelo de Bäverstam and Othaz) o una velocidad de relajación $\Gamma_{r}$ (modelo de Relajación Unidireccional) del orden $10^{2} \mathrm{MHz}$. Por otro lado, para el tipo de sistemas que estudiamos en este trabajo se tiene $\Delta \omega<<\Gamma_{r}$, con lo que $b_{n}$ resulta despreciable. Por lo tanto, en estos rangos los factores de perturbación de ambos modelos resultan equivalentes. Finalmente, por la simplicidad del modelo y por los rangos de frecuencias cuadrupolares medidos en el presente trabajo utilizaremos el modelo de Bäverstam y Othaz para el estudio de las interacciones dinámicas medidas.

Por ultimo, el modelo de Bäverstam y Othaz presenta la ventaja que solo define dos parámetros dinámicos lo que lo hace muy simple, útil y altamente eficiente para ajustar espectros PAC con atenuación dinámica. Por otro lado, en el modelo de relajación unidireccional es necesario definir un número determinado de GCEs iniciales y un GCE final, los cuales son observados por la sonda $\left({ }^{111} \mathrm{In} \rightarrow\right)^{111} \mathrm{Cd}$ con una velocidad de transición (única para todas las transiciones) y en una ventana de tiempo muy pequeña de tiempo ( $1 \mathrm{~ns}$ ). Este hecho hace que el modelo de relajación unidireccional sea 
poco eficiente al ajustar espectros PAC con relajación dinámica debido a la dificultad de elegir satisfactoriamente el número y tipo de GCEs iniciales en una ventana de tiempo del orden de 1 ns en los espectros R(t).

\section{Referencias}

[Carlson 1966] T.A. Carlson, W.E. Hunt y M.O. Krause, Phys. Rev. 151, 41 (1966).

[Bäverstam, 1972] U. Bäverstam, R. Othaz, N. De Sousa and B. Ringström, Nucl. Phys. A186, 500 (1972).

[Abragam, 1953] A. Abragam and R. V. Pound, Phys. Rev. 92, 943 (1953).

[Lupascu, 1996] D. Lupascu, S. Habenicht, K.P. Lieb, M. Neubauer, M. Uhrmacher, and T. Wenzel, Phys. Rev. B 54871 (1996).

[Bibiloni 1985] A.G. Bibiloni, C.P. Massolo, J. Desimoni, L.A. Mendoza-Zelis, F. H. Sanchez, A.F. Pasquevich, L. Damonte and A.R. Lopez-García, Phys. Rev. B 322393 (1985). 


\section{Capítulo Nº}

\section{Métodos de cálculo}

\section{4-1 Modelo de cargas puntuales}

Dado un sólido de volumen y densidad de carga conocidos, entonces el tensor gradiente de campo eléctrico $V_{\mathrm{ij}}$ generado en el origen de coordenadas por la densidad de carga del cristal puede expresarse como:

$$
V_{i j}=\frac{1}{4 \pi \varepsilon_{0}} \int_{\substack{\text { vol } \\ \text { cristal }}} \frac{\rho(\vec{r})\left(3 x_{i} x_{j}-\delta_{i j} r^{2}\right)}{r^{5}} d^{3} r,
$$

donde $\rho(\vec{r})$ representa la densidad de carga total en la posición $\vec{r}$ del cristal y la integral se extiende a todo su volumen. Es decir que para determinar el tensor GCE en el sólido solo hace falta encontrar $\rho(\vec{r})$ en todo el cristal.

El modelo más simple que determina la densidad de carga $\rho(\vec{r})$, es el modelo de cargas puntuales (PCM por sus siglas en inglés). En el marco de este modelo, el cristal está formado por cargas puntuales cuyas posiciones coinciden con los sitios atómicos de red. De esta manera, la integral (4.1) deviene en una sumatoria debido a la suma de distribuciones delta de Dirac de la densidad de carga. Cada sitio de red puede tener una carga $\mathrm{Ne}$, donde $\mathrm{N}$ es un número entero positivo o negativo según sea la carga del ión. En general, para un cristal iónico de un óxido binario cuya fórmula estequiométrica sea $R_{X}^{N+} O_{Y}^{2-}$ la carga para los sitios de anión es $-2 e$, mientras que para los sitios de catión es $N=+2 e Y / X$, de manera tal de asegurar la neutralidad eléctrica del cristal. Entonces, en el modelo PCM la forma general de la densidad de carga queda:

$$
\rho(\vec{r})=\sum_{k_{\text {ion }}} N_{k_{\text {ion }}} e \delta\left(\vec{r}-\vec{r}_{k_{\text {ion }}}\right)
$$

A partir de la expresión (4.1), se obtiene el tensor GCE en el marco del modelo de cargas puntuales:

$$
V_{i j}^{\text {red }}=\frac{1}{4 \pi \varepsilon_{0}} \sum_{k_{\text {ion }}} \frac{N_{k}|e|\left(3 x_{i k} x_{j k}-\delta_{i j} r_{k}^{2}\right)}{r_{k}^{5}} .
$$


La sumatoria se extiende a todos los iones $k$ de la red cristalina, excluyendo el núcleo sonda que por simplicidad se coloca en el origen de coordenadas. En (4.3) $x_{i k}, X_{j k}, r_{k}$ simbolizan coordenadas y distancia del ión k-ésimo al átomo sonda, respectivamente.

Conociendo los parámetros de red del cristal $(a, b, c)$, los ángulos $\alpha, \beta, \gamma$ y las posiciones de todos los átomos en la celda unidad, la red cristalina queda completamente determinada. De este modo se puede calcular cada componente $V_{i j}$ del tensor GCE en cada sitio de la red a partir del modelo PCM. Los cálculos que se presentan en este trabajo se realizaron con el programa GITSUM [GITSUM 1988]. Este programa lee los parámetros de la red cristalina y las posiciones atómicas, generando la red por traslación de la celda unidad. Luego de la construcción de la red, el programa toma una esfera de radio $R_{C}$, donde dicho parámetro es el radio de corte del cálculo. El programa calcula cada elemento de matriz $V_{i j}$ sumando directamente las contribuciones de cada ión de acuerdo a la ecuación (4.3). Una subrutina del programa [EIGEN 1962] diagonaliza la matriz del tensor, dando como resultado los ejes principales del tensor GCE, sus elementos diagonales $V_{\text {ii }}$ y el parámetro de asimetría $\eta$.

Cabe aclarar que al excluir el átomo sonda en la sumatoria (4.3), no se tiene en cuenta la influencia del carozo electrónico de dicho átomo sobre el valor del tensor GCE. Se puede incluir la contribución del carozo al GCE introduciendo en el cálculo un factor que depende del átomo sonda y se denomina "factor de anti-apantallamiento de Sternheimer” $\left(\gamma_{\infty}\right)$, [Sternheimer 1953]. Como la intensidad de la interacción cuadrupolar nuclear producida por un GCE externo a la sonda se ve modificada (en la mayoría de los casos amplificada) por los electrones ligados al núcleo sonda, entonces, este factor tiene en cuenta esta modificación. El factor $\gamma_{\infty}$ es negativo para la mayoría de los iones con capa cerrada, y su valor (para átomos medianos y pesados) oscila entre -10 y -80. Debemos destacar que este factor, calculado así por Sternheimer, depende sólo de la estructura electrónica del átomo-sonda y es independiente de la magnitud o simetría del campo externo y de la interacción de dicho átomo con el resto de los iones de la red. Es decir, este factor supone que los electrones de carozo mantienen su simetría de carga esférica frente al GCE, sin tener en cuenta la polarización y/o hibridación que puedan sufrir como consecuencia de su interacción con el resto de los iones y electrones de la red. El valor reportado para el $\mathrm{Cd}^{+2}$ es $\gamma_{\infty}=-29,27$, mientras 
que para el $\mathrm{Ta}^{5+}, \gamma_{\infty}=-61,42$ [Feiock 1969]. Considerando este término, el GCE en el sitio del átomo-sonda viene dado por:

$$
V_{i j}=\left(1-\gamma_{\infty}\right) V_{i j}^{r e d}
$$

En la expresión (4.4) el primer término representa la contribución de la red de iones al gradiente en el sitio del núcleo sonda calculada a partir de la ecuación (4.3) del modelo PCM, y el segundo término es la contribución de los electrones de carozo al GCE con las hipótesis de Sternheimer.

En el marco de este modelo, la frecuencia cuadrupolar $\omega_{Q}$ y el parámetro de asimetría $\eta$ se relacionan con las componentes $V_{i i}$ a través de:

$$
\omega_{Q}=\frac{e Q}{40 \hbar}\left(1-\gamma_{\infty}\right) V_{33}^{r e d}, \quad \eta=\frac{V_{11}^{r e d}-V_{22}^{\text {red }}}{V_{33}^{\text {red }}},
$$

siendo $Q$ el momento cuadrupolar nuclear del núcleo-sonda en su estado intermedio I de la cascada $\gamma-\gamma$.

Por último, este modelo no tiene en cuenta el hecho que la sonda sea un átomo nativo de la estructura o una impureza debido a que se basa en sumas de red y solo considera la contribución al GCE de parte de los electrones de la sonda (a través del factor de Sternheimer), y los supone no interactuantes con el resto de los electrones del cristal. Es decir, bajo estas hipótesis, el único efecto de los electrones del átomo-sonda es apantallar o anti-apantallar el GCE. Por lo tanto, este modelo no incluye posibles relajaciones que pueda introducir el átomo sonda, ni tampoco la aparición de estados de impureza por debajo de la banda de conducción o por encima de la banda de valencia, ni la interacción de los electrones del átomo-sonda con su entorno.

\section{4-2 Teoría de la Funcional Densidad}

El sólido es un conjunto de partículas cargadas positivamente (núcleos) y cargadas negativamente (electrones) interactuando entre sí. Supongamos que se tiene un sistema de $N$ átomos idénticos de número atómico $Z$, entonces debemos resolver un problema de $N+Z N$ partículas cuánticas interactuando entre sí. El hamiltoniano del sistema puede expresarse según: 


$$
\hat{H}=-\frac{\hbar^{2}}{2} \sum_{i} \frac{\nabla^{2} \vec{R}_{i}}{M_{i}}-\frac{\hbar^{2}}{2} \sum_{i} \frac{\nabla^{2} \vec{r}_{i}}{m_{e}}-\frac{1}{4 \pi \varepsilon_{0}} \sum_{i j} \frac{e^{2} Z_{i}}{\left|\vec{R}_{i}-\vec{r}_{j}\right|}+\frac{1}{8 \pi \varepsilon_{0}} \sum_{i \neq j} \frac{e^{2}}{\left|\vec{r}_{i}-\vec{r}_{j}\right|}+\frac{1}{8 \pi \varepsilon_{0}} \sum_{i \neq j} \frac{e^{2} Z_{i} Z_{j}}{\left|\vec{R}_{i}-\vec{R}_{j}\right|},
$$

donde $M_{i}$ es la masa del núcleo situado en la posición $\vec{R}_{i}$, y $m_{e}$, la masa del electrón de coordenadas $\vec{r}_{i}$.

El primer término de (4.6) es el operador energía cinética aplicado a todos los núcleos del sistema y el segundo es el mismo operador pero aplicado al total de electrones del sólido. Los restantes tres términos representan la interacción coulombiana electrón-núcleo, electrón-electrón y núcleo-núcleo, respectivamente.

Debido a la imposibilidad de encontrar la solución exacta del problema, esto es, los autovalores y autovectores del hamiltoniano (4.6), es necesario realizar determinadas aproximaciones con el fín de simplificar el problema y poder resolverlo.

Debido a que la masa del protón $m_{p}$ cumple $m_{p} \cong 2000 m_{e}$, por lo que los núcleos se moverán lentamente respecto a los electrones. Por lo tanto, vamos a suponer que los núcleos se mantienen fijos en los sitios de red. Esta es la primera aproximación que simplifica el problema y se denomina aproximación de Born-Oppenheimer. Finalmente, hemos reducido el problema a un sistema de $N Z$ electrones interactuantes moviéndose en el potencial externo generado por los núcleos. Ahora el hamiltoniano toma la forma:

$$
\hat{H}=\hat{T}+\hat{V}+\hat{V}_{e x t},
$$

donde $\hat{T}$ es la energía cinética de los electrones, $\hat{V}$ es la energía potencial de interacción entre electrones y $\hat{V}_{\text {ext }}$ es la energía potencial debida a la interacción de los electrones con el potencial externo generado por los núcleos. El problema de encontrar los autovectores y autovalores de este hamiltoniano simplificado sigue siendo muy complicado por lo que haremos otras aproximaciones.

El método de Hartree-Fock permite obtener un hamiltoniano más accesible para resolverlo. Si bien este método es muy bueno para tratar átomos y moléculas, resulta obsoleto para describir sistemas periódicos infinitos como un sólido.

El método fue propuesto originariamente por Hartree en 1928 y postula que la función de onda de un sistema de muchos electrones puede expresarse como el producto de las funciones de onda de cada electrón, esto es: 


$$
\Phi=\prod_{i} \varphi_{i}\left(\vec{r}_{i}\right)
$$

Cada una de estas funciones de onda satisface una ecuación de Schrödinger del tipo:

$$
\left[-\frac{\hbar^{2}}{2} \nabla^{2}+V_{i}\left(\vec{r}_{i}\right)\right] \varphi_{i}\left(\vec{r}_{i}\right)=\varepsilon_{i} \varphi_{i}\left(\vec{r}_{i}\right),
$$

donde el primer término está asociado a la energía cinética del i-ésimo electrón y el segundo es el potencial externo generado por los núcleos y el resto de los electrones del sistema. En este método (de Hartree) los electrones son tratados como partículas distinguibles. Para que sean considerados como fermiones todavía falta considerar el método de Hartree-Fock, donde se tiene en cuenta el principio de exclusión de Pauli, esto es el carácter fermiónico de los electrones, antisimetrizando la función de onda $\Phi$ mediante el determinante de Slater.

$$
\Phi=\frac{1}{\sqrt{N !} \mid}\left|\begin{array}{cccc}
\varphi_{1}\left(\vec{r}_{1}\right) & \ldots & \ldots & \varphi_{1}\left(\vec{r}_{N}\right) \\
\ldots & \ldots & \ldots & \ldots \\
\ldots & \ldots & \ldots & \ldots \\
\varphi_{N}\left(\vec{r}_{1}\right) & \ldots & \ldots & \varphi_{N}\left(\vec{r}_{N}\right)
\end{array}\right|
$$

De esta manera, el método de Hartree-Fock tiene en cuenta el intercambio entre los electrones, pero aún carece del efecto de correlación electrónica.

Otra manera de resolver el Hamiltoniano del sólido es encontrar la densidad electrónica del sistema $\rho(\vec{r})$. Este método es conocido como Teoría de la Funcional Densidad (DFT, por sus siglas en inglés) y permite expresar una ecuación de Schrödinger con una función de onda de $3 \mathrm{~N}$ variables espaciales como una funcional de la densidad electrónica $\rho(\vec{r})$ con solo 3 variables.

En el año 1927, siguiendo los trabajos pioneros de Hartree, en forma independiente Thomas [Thomas, 1926] y Fermi [Fermi, 1928] construyeron un modelo semiclásico para átomos pesados aislados en términos de la densidad electrónica $\rho(r)$ del estado fundamental. Esta teoría fue generalizada por Dirac [Dirac, 1930] razón por la cual se la conoce como “Teoría de Thomas-Fermi-Dirac” y es la precursora de la actual DFT. 
En 1964, partiendo de la “Teoría de Thomas-Fermi-Dirac”, Hohenberg y Kohn demostraron que la energía total $\mathrm{E}$ de un sistema de electrones interactuando con el potencial externo creado por los núcleos localizados en los sitios de una red, puede expresarse como una funcional de la densidad electrónica $\rho(r)$ [Hohenberg, 1964]:

$$
E=E[\rho(\vec{r})],
$$

Hohenberg y Kohn demostraron además que la densidad electrónica $\rho(\overrightarrow{\mathrm{r}})$ correcta del estado fundamental es aquella que minimiza $E[\rho(\vec{r})]$. Esto se conoce como Teorema de Hohenberg y Kohn y es la base de la DFT.

El teorema demuestra que la densidad del estado fundamental es la que minimiza la energía pero no da información de las características de la densidad, solo da cuenta del carácter de potencial externo que tiene el potencial coulombiano de los núcleos, y de la misma forma, el potencial de interacción entre los electrones, por ende no permite obtener la forma de $E[\rho(r)]$. Es por esto que no se evalúa la utilidad de dicha teoría sino la utilidad de los diferentes métodos de cálculo basados en la DFT, es decir, cuán buenas sean las predicciones dependerá de cuán buenas sean las aproximaciones del método. En la práctica, estas aproximaciones se realizan escribiendo la funcional desconocida $E[\rho(r)]$ como la suma de la energía total de Hartree $E_{H}[\rho(\vec{r})]$ y un término llamado de correlación e intercambio, $E_{x c}[\rho(r)]$ [Hohemberg, 1964]:

$$
E[\rho(\vec{r})]=E_{H}[\rho(\vec{r})]+E_{x c}[\rho(\vec{r})],
$$

donde la energía de Hartree viene dada por:

$$
E_{H}[\rho(\vec{r})]=T[\rho(\vec{r})]+E_{e n}[\rho(\vec{r})]+E_{n n}[\rho(\vec{r})]+E_{e e}[\rho(\vec{r})] .
$$

$T[\rho(\vec{r})]$ representa la energía cinética de los electrones, $E_{e n}[\rho(\vec{r})]$ es la energía coulombiana de interacción entre los electrones y los núcleos, $E_{n n}[\rho(\vec{r})]$ es la energía de interacción entre los núcleos y $E_{e e}[\rho(\vec{r})]$ es la energía de interacción electrónelectrón cuya forma explícita es: 


$$
E_{e e}[\rho(\vec{r})]=\frac{e^{2}}{2} \int d^{3} r d^{3} r^{\prime} \frac{\rho(\vec{r}) \rho\left(\vec{r}^{\prime}\right)}{\left|\vec{r}-\vec{r}^{\prime}\right|}
$$

Para el término de correlación e intercambio se utiliza la Aproximación de Densidad Local (LDA). Esta aproximación supone que $E_{x c}[\rho(\vec{r})]$ se puede escribir en términos de una densidad local $\varepsilon_{x c}[\rho(\vec{r})]$ en la forma:

$$
E_{x c}=\int \varepsilon_{x c}[\rho(\vec{r})] \rho(\vec{r}) d^{3} r
$$

Por lo general se elige $\varepsilon_{x c}[\rho(\vec{r})]$ de manera que reproduzca la energía de un gas de electrones [Perdew, 1992].

Existe otra aproximación conocida como Aproximación de Gradiente Generalizado (GGA), que básicamente establece que $\varepsilon_{x c}[\rho(\vec{r})]$ depende de la densidad $\rho(\vec{r})$ y de la magnitud del gradiente de la densidad $|\nabla \rho(\vec{r})|$, esto es $\varepsilon_{x c}[\rho(\vec{r}),|\nabla \rho(\vec{r})|][$ Perdew,1996].

Kohn y Sham demostraron a partir de la teoría variacional, que la funcional $E[\rho(\vec{r})]$ se hace mínima para la densidad de estados calculada con las funciones de onda electrónicas que son soluciones de las ecuaciones de Kohn y Sham (K-S):

$$
(T+V(\vec{r})) \vartheta_{i}(\vec{r})=\left(T+V_{e n}(\vec{r})+V_{H}(\vec{r})+V_{x c}(\vec{r})\right) \vartheta_{i}(\vec{r})=\varepsilon_{i} \vartheta_{i}(\vec{r})
$$

donde $\vartheta_{\mathrm{i}}$ y $\varepsilon_{\mathrm{i}}$ son las autofunciones y autovalores de la ecuación de Schrödinger de partícula independiente, $\mathrm{T}$ es el operador de energía cinética, $\mathrm{V}_{\mathrm{en}}$ es el potencial de interacción coulombiana entre electrones y núcleos, $\mathrm{V}_{\mathrm{H}}$ es el potencial de Hartree y $\mathrm{V}_{\mathrm{xc}}$ es el potencial de correlación e intercambio, cuyas formas funcionales son:

$$
V_{H}(\vec{r})=e^{2} \int d^{3} r^{\prime} \frac{\rho\left(\vec{r}^{\prime}\right)}{\left|\vec{r}-\vec{r}^{\prime}\right|} \quad ; \quad V_{x c}(\vec{r})=\frac{\delta E_{x c}(\rho)}{\delta \rho(\vec{r})} .
$$

La forma explícita de la densidad electrónica viene dada por una suma de Fermi sobre los estados ocupados:

$$
\rho(\vec{r})=\sum_{i} \vartheta_{i}^{*}(\vec{r}) \vartheta_{i}(\vec{r})
$$


De este modo vemos que dada una densidad $\rho(\vec{r})$, con ésta se calculan los potenciales de interacción (4.17) y luego los orbitales $\vartheta_{i}(\vec{r})$ con (4.16), para nuevamente obtener $\rho(\vec{r})$ a partir de (4.18), y así iterativamente hasta lograr una autoconsistencia cuyo error sea menor que el que se pretende para los autovalores de energía. Cabe aclarar que si bien el cálculo puede realizarse con poca dispersión en las determinaciones de las funciones de onda y por ende en la densidad y en la energía, esto no tiene ninguna relación con la capacidad que tiene el método en predecir los resultados experimentales. Esta capacidad está relacionada con las aproximaciones y suposiciones que se hagan sobre el potencial $\mathrm{V}(\vec{r})$ y con la base de funciones de onda que se elija para desarrollar los orbitales $\vartheta_{i}(\vec{r})$.

A continuación, describiremos el método APW+lo (Augmented Plane Wave plus local orbital) utilizado para resolver las ecuaciones de Kohn y Sham. Para llegar a comprender este método vamos a describir los métodos APW, LAPW y LAPW+LO necesarios para abordar el APW+lo.

\section{4-2-1 Método APW}

El método APW (Augmented Plane Wave) fue desarrollado por Slater en 1937 [Slater 1937]. En este método para describir el sólido se consideran dos regiones, una región esférica centrada en los entornos de los sitios atómicos donde el potencial y las funciones de onda varían fuertemente, y la otra es la región intersticial donde el potencial y las autofunciones se comportan suavemente. El radio de las esferas centradas en los sitios atómicos se elige de modo tal que dichas esferas no se superpongan. Esto se esquematiza en la Figura 4.1.

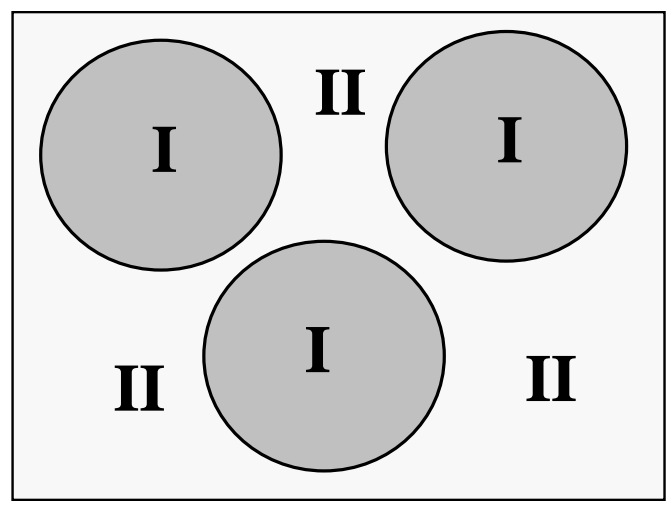

Figura 4.1: Esquema de las regiones definidas en el método APW
En la región I se utiliza como base de funciones de onda para el desarrollo de las autofunciones $\vartheta_{i}(\vec{r})$, funciones de onda en coordenadas esféricas, soluciones de la ecuación de Schrödinger independiente del tiempo:

$$
\vartheta_{\vec{G}}^{\vec{k}}(\vec{r})=\sum_{l, m} A_{l, m}^{\vec{G}+\vec{k}} u_{l}(r) Y_{l, m}(\breve{r})
$$


donde $Y_{l, m}(\breve{r})$ son armónicos esféricos y $u_{l}(r)$ es la solución regular de la ecuación radial de Schrödinger:

$\left\{-\frac{d^{2}}{d r^{2}}+\frac{l(l+1)}{r^{2}}+V(r)-E_{l}\right\} r u_{l}(r)=0$,

mientras que en la región II se utiliza un desarrollo en ondas planas:

$$
\vartheta_{\vec{G}}^{\vec{k}}(\vec{r})=\frac{1}{\sqrt{\Omega}} e^{i(\vec{G}+\vec{k}) \vec{r}},
$$

donde $\vec{G}$ son los vectores de la red recíproca y $\vec{k}$ es el vector de onda dentro de la primer zona de Brillouin. Entonces, la solución de las ecuaciones K-S es expandida en esta base combinada:

$$
\Psi_{\vec{k}}^{n}=\sum_{\vec{G}} C_{\vec{G}}^{n \vec{k}} \vartheta_{\vec{G}}^{\vec{k}}(\vec{r})
$$

Para garantizar la continuidad de las funciones $\vartheta_{\vec{G}}^{\vec{k}}(\vec{r})$ en los bordes de las esferas atómicas, las ondas planas son expandidas en armónicos esféricos y se hacen coincidir los coeficientes de componentes $l, m$ en los bordes. Finalmente, los coeficientes $A_{k, m}^{\vec{k}+\vec{G}}$ de (4.19) quedan [Cottenier 2002]:

$$
A_{l, m}^{\vec{k}+\vec{G}}=\frac{4 \pi i^{l}}{\sqrt{\Omega} u_{l}\left(E_{l}, R_{i}\right)} J_{l}\left(|\vec{k}+\vec{G}| R_{i}\right) Y_{l, m}^{*}(\vec{k}+\vec{G}),
$$

donde $\mathrm{J}_{1}$ es la función de Bessel de orden l. Los $A_{k, m}^{\vec{k}+\vec{G}}$ son los coeficientes variacionales en el método APW. 
En el método APW las funciones son soluciones de la ecuación radial de Schrödinger solo para la energía $\mathrm{E}_{\mathrm{l}}$, de esta manera el cálculo debe realizarse para diferentes valores de $\mathrm{E}_{\mathrm{l}}$, con lo que se pierde libertad variacional.

\section{4-2-2 Método FP-LAPW}

En el método LAPW se elimina el inconveniente de que las funciones de onda solución de la ecuación de Schrödinger radial solo lo son para la energía $E_{l}$ expresando las funciones de la base como combinación lineal de las funciones $u_{l}(\vec{r}) Y_{l m}(\breve{r})$ y de sus derivadas con respecto a la energía $\dot{u}_{l}(\vec{r})$. Las funciones radiales $u_{l}(\vec{r})$ son las deducidas en el método APW, mientras que sus derivadas $\dot{u}_{l}(\vec{r})$ satisfacen:

$\left[-\frac{d^{2}}{d r^{2}}+\frac{l(l+1)}{r^{2}}+V(r)-E_{l}\right] r \dot{u}_{l}(r)=r u_{l}(r) \quad$.

Estas funciones ligadas a las soluciones de onda plana para garantizar la continuidad en los bordes de las esferas, son las funciones LAPW.

En términos de esta base, las autofunciones en las dos regiones son:

$$
\vartheta_{\vec{G}}^{\vec{k}}(\vec{r})= \begin{cases}\sum_{l, m}\left[A_{l, m}^{\vec{k}+\vec{G}} u_{l}\left(r, E_{l}\right)+B_{l, m}^{\vec{k}+\vec{G}} \dot{u}_{l}\left(r, E_{l}\right)\right] Y_{l, m}(\breve{r}) & \text { Región I } \\ \frac{1}{\sqrt{\Omega}} e^{i(\vec{G}+\vec{k}) r} & \text { Región II . }\end{cases}
$$

Al igual que en el método APW, las funciones en la región intersticial son ondas planas, mientras que en las esferas atómicas las funciones LAPW tienen un mayor grado de libertad variacional.

Supongamos que hemos resuelto las ecuaciones K-S en el marco de la DFT, entonces conocemos la densidad $\rho(\vec{r})$ del estado fundamental del sistema. Entonces, a partir de esta hipótesis, veamos como obtener las propiedades del sistema bajo estudio. En este método, en forma análoga a la densidad de carga, el potencial se expande en la forma [Blaha 1988]: 
$V(\vec{r})= \begin{cases}\sum_{l, m} V_{l, m}(r) Y_{l, m}(\vec{r}) & \text { Región I } \\ \sum_{K} V_{K} e^{i \vec{K} . \vec{r}} & \text { Región II . }\end{cases}$

Al no realizarse aproximaciones de forma al potencial, este método recibe el nombre de Full-Potential (FP), logrando que el FP-LAPW sea ideal para calcular el tensor GCE debido a que es un observable muy sensible a pequeños cambios en la simetría de la densidad de carga.

Anteriormente no se utilizaba la expansión (4.26) para el potencial cristalino, sino la aproximación de muffin-tin, que consiste en tomar únicamente los términos con $l=m=K=0$ en (4.25). Esto equivale a tomar un promedio esférico del potencial dentro de
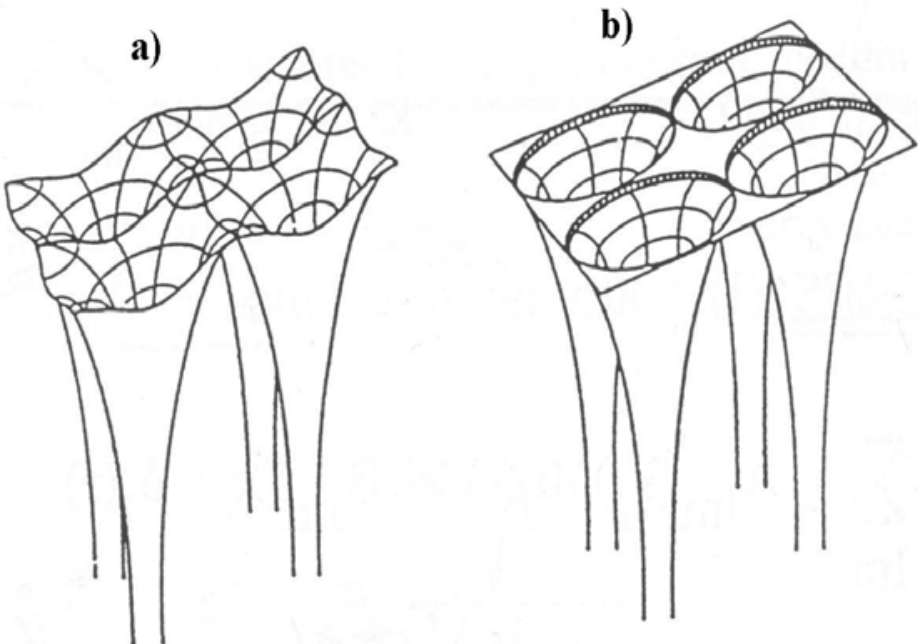

Figura 4.2: Potencial V(r) correspondiente a una red cuadrada bidimensional: (a) FPAPW+lo. (b) “muffin-tin". las esferas atómicas (sin dependencia angular) y un promedio en la región intersticial (ver Figura 4.2). Esta aproximación es muy buena para estructuras compactas (FCC o HCP), aceptable para las BCC pero pierde confiabilidad para estructuras abiertas, asimétricas o de baja coordinación.

A partir del potencial autoconsistente se pueden obtener las componentes del tensor GCE como sigue [Blaha 1989]:

$$
\begin{aligned}
V_{Z Z} & =\sqrt{\frac{5}{4 \pi}} 2 \lim _{r \rightarrow 0}\left[V_{20}(r)\right] \\
V_{Y Y} & =\sqrt{\frac{5}{4 \pi}} 2 \lim _{r \rightarrow 0}\left[-\sqrt{3} V_{22}(r)-V_{20}(r)\right] \\
V_{X X} & =\sqrt{\frac{5}{4 \pi}} 2 \lim _{r \rightarrow 0}\left[\sqrt{3} V_{22}(r)-V_{20}(r)\right],
\end{aligned}
$$


donde el potencial puede ser calculado a partir de la densidad total de carga del sistema. Los coeficientes $V_{l, m}$ del desarrollo del potencial se obtienen vía las condiciones de contorno [Weinert 1981]. Entonces, el valor de la componente $l=2, m=0$ en $r=0$ (análogamente para $l=2, m=2$ ) puede obtenerse a partir de la siguiente expresión [Weiner 1981, Schwarz 1990]:

$V_{20}(r=0)=\frac{4 \pi}{5} \int_{0}^{R_{t}} \frac{\rho_{20}}{r^{3}} r^{2} d r-\frac{4 \pi}{5} \int_{0}^{R_{t}} \frac{\rho_{20}}{r^{3}}\left(\frac{r}{R_{i t}}\right)^{5} r^{2} d r+4 \pi \sum_{K} V(K) J_{2}\left(K R_{i}\right) Y_{20}(\breve{K})$,

donde $J_{2}\left(K R_{i}\right)$ es la función esférica de orden 2. Entonces, a partir de DFT hemos obtenido el tensor GCE sin definir factores correctivos arbitrarios como ser el factor de anti-apantallamiento de Sternheimer utilizado en el marco de PCM. El primer término de (4.28) es la integral de la densidad de carga multiplicada por el polinomio de Legendre de orden 2, dividido por $r^{3}$ e integrada sobre la esfera atómica. Este término se denomina de valencia porque es originado en la densidad electrónica no esférica de los electrones de valencia y semi-carozo dentro de la esfera atómica, cabe destacar que la densidad electrónica puramente de carozo no contribuye al GCE debido a que suponemos que simetría es esférica. El segundo término aparece al resolver el problema de condición de contorno en el borde las esferas atómicas y garantiza la continuidad del potencial en $r=R_{i}$. El tercer término se origina en la densidad de carga exterior a las esferas atómicas. La suma de los dos últimos términos es la contribución de red. Esta división en dos partes para un radio de esfera atómica dado es exacta pero difiere de la división de valencia y de red hecha en PCM debido a que en este último modelo no se tiene en cuenta los efectos de hibridación de los electrones de la sonda y los átomos vecinos.

Veamos la forma del desarrollo de $\rho(\vec{r})$, la cual puede escribirse como sigue:

$$
\rho_{L, M}(r)=\sum_{E<E_{F}} \sum_{l, m} \sum_{l^{\prime}, m^{\prime}} R_{l, m}(r) R_{l^{\prime}, m^{\prime}}(r) G_{L, l, l^{\prime}}^{M, m, m^{\prime}}
$$

donde las funciones G son los llamados “números de Gaunt” (integral sobre productos de armónicos esféricos). Debido a que en el GCE solo contribuye la componente $\rho_{20}$, entonces las contribuciones para $L=0$ y $M=0$ son $l=l^{\prime}=1$ y $l=l^{\prime}=2$ (y en mucho menor 
medida $\left.l=0, l^{\prime}=2\right)$ darán números de Gaunt no nulos. Estas son las llamadas $p-p, d-d(\mathrm{y}$ $s-d)$ al GCE [Blaha, 1989].

Otro observable que puede obtenerse es la fuerza resultante actuante sobre cada átomo del sólido. A partir de estas fuerzas es posible determinar las posiciones atómicas de equilibrio, esto se hace calculando la superficie de energía para todas las configuraciones posibles de las posiciones, es decir, mapeando dicha superficie (calculada con DFT) como función de las posiciones con la finalidad de obtener el mínimo. La dificultad de este procedimiento es que se vuelve ineficiente con el aumento del número $N$ de átomos de la celda unidad puesto que la dimensión de la superficie de energía crece como $N^{2}$, aumentando el número de cálculos considerablemente. El procedimiento que utiliza el método es calcular las fuerzas sobre cada átomo y desplazar los mismos en función de estas fuerzas hasta que sean menores a un valor de tolerancia, esto hace que el número de cálculos disminuya considerablemente. La fuerza $\boldsymbol{F}_{\boldsymbol{i}}$ sobre el átomo $i$ se calcula según:

$$
\vec{F}_{i}=-\frac{d E_{\text {total }}}{d \vec{r}_{i}}
$$

\section{4-2-3 Método FP-LAPW+LO}

El método FP-LAPW es el que trata los estados de valencia, los cuales se escapan de las esferas de muffin-tin y son los que participan de los enlaces químicos con los otros átomos. Además, los estados de carozo, los cuales se encuentran por completo dentro de la esfera de muffin-tin muy ligados al núcleo y no participan de los enlaces químicos, son considerados como si estuvieran en sus átomos libres pero sujetos al potencial debido a los estados de valencia. Sin embargo existen estados que no son ni totalmente de carozo ni totalmente de valencia, estos se denominan estados de semicarozo. Cuando existen más de un estado, de los cuales uno es de semi-carozo, con el mismo número cuántico $l$ pero diferente número principal $n$ aparece el problema de la elección de la energía de linearización. Por ejemplo, debido a la hibridización de orbitales, el Fe en el Fe-bcc (estructura cúbica centrada en el cuerpo) tiene una cantidad no despreciable de estados de valencia de carácter $4 p$ aproximadamente $0.2 \mathrm{Ry}(2.7 \mathrm{eV}$ ) por debajo del nivel de Fermi. Pero los estados 3p, los cuales están 4.3 Ry (58.5 eV) por debajo de $E_{F}$ tampoco están confinados completamente en el carozo, este estado de 
valencia de baja energía se denomina estado de semi-carozo. La dificultad en este tipo de sistemas es la elección de la energía de linearización $E^{F e}(l=1)$, es decir, en LAPW ni una energía cercana al nivel $3 p$, o al nivel $4 p$, ni un valor intermedio termina siendo una elección óptima. Dicho problema se resuelve agregando otro tipo de funciones llamadas orbitales locales (LO) a la base de funciones LAPW.

$\vartheta_{l, m}^{\alpha^{\prime}, L O}(\vec{r})=\left\{\begin{array}{cc}{\left[A_{l, m}^{\alpha^{\prime}, L O} u_{l}^{\alpha^{\prime}}\left(r^{\prime}, E_{1, l}^{\alpha^{\prime}}\right)+B_{l, m}^{\alpha^{\prime}, L O} \dot{u}_{l}^{\alpha^{\prime}}\left(r^{\prime}, E_{1, l}^{\alpha^{\prime}}\right)+C_{l, m}^{\alpha^{\prime}, L O} u_{l}^{\alpha^{\prime}}\left(r^{\prime}, E_{2, l}^{\alpha^{\prime}}\right)\right] Y_{l, m}\left(r^{\prime}\right) \text { RegiónI }} \\ 0 & \text { RegiónII }\end{array}\right.$

El símbolo ' indica que se consideran todos los átomos de este tipo de la celda unidad (no solo los inequivalentes). Un orbital local se define nulo en la región intersticial y en las esferas de muffin-tin de los otros átomos. Dentro de las esferas de muffin-tin del átomo $\alpha^{\prime}$ se usan las mismas funciones $u_{l}^{\alpha^{\prime}}\left(r^{\prime}, E_{1, l}^{\alpha^{\prime}}\right)$ y $u_{l}^{\alpha^{\prime}}\left(r^{\prime}, E_{1, l}^{\alpha^{\prime}}\right)$ de la base LAPW con un valor para la energía de linearización $E_{1, l}^{\alpha^{\prime}}$ correspondiente al mayor de los estados de valencia de los posibles estados con el mismo l pero distinto número cuántico principal $n$. Por otro lado, el menor de estos estados posee un pico a una energía $E_{2, l}^{\alpha^{\prime}}$, dicho estado se comporta como el de un átomo libre por lo que una función radial $u_{l}^{\alpha^{\prime}}\left(r^{\prime}, E_{2, l}^{\alpha^{\prime}}\right)$ será suficiente para describirlo. Otra característica de los orbitales locales es que no se conectan con las ondas planas existentes en las regiones intersticiales por lo que no son función de $\vec{k}$ o $\vec{G}$.

\section{4-2-4 Método APW+lo}

\section{Funciones de base del método APW+lo "puro":}

Como se ha dicho, el problema con el método APW es la dependencia de las funciones de la base con la energía. Esta dependencia pudo ser removida en el LAPW+LO a expensas de tener una tamaño mayor de la base debido a que a la base LAPW se le agregan determinados orbitales locales. En cambio, en el método APW+lo las funciones de la base son independientes de la energía y la base tiene el mismo 
tamaño que el método APW como veremos a continuación. Por todo esto es que el método APW+lo combina lo mejor del APW y del LAPW+LO.

El conjunto de funciones de la base de APW+lo combina dos tipos funciones. Las primeras son funciones APW con un conjunto de energías $E_{l}^{\alpha}$, las cuales se definen como:

$$
\vartheta_{\vec{G}}^{k}(\vec{r})= \begin{cases}\sum_{l, m} A_{l, m}^{\alpha, \vec{k}+\vec{G}} u_{l}\left(r^{\prime}, E_{l}^{\alpha}\right) Y_{l, m}\left(\vec{r}^{\prime}\right) & \text { Región I } \\ \frac{1}{\sqrt{\Omega}} e^{i(\vec{G}+\vec{k}) r} & \text { Región II , }\end{cases}
$$

donde $u_{l}\left(r^{\prime}, E_{l}^{\alpha}\right)$ es la solución regular de la ecuación radial de Schrödinger para la energía $E_{l}^{\alpha} ; \vec{G}$ son vectores de la red recíproca y $\vec{k}$ es el vector de onda dentro de la primer zona de Brillouin.

Pero como a energías fijas esta base no da una buena descripción de las autofunciones, entonces, debemos aumentar el conjunto de las funciones de la base con otro tipo de funciones. Estas funciones son orbitales locales diferentes a las utilizadas en el método LAPW+LO por lo que las señalaremos con "lo" en lugar de "LO" y se definen según:

$$
\vartheta_{l, m}^{\alpha^{\prime}, l o}(r)=\left\{\begin{array}{cl}
{\left[A_{l, m}^{\alpha^{\prime}, l o} u_{l}^{\alpha^{\prime}}\left(r^{\prime}, E_{l}^{\alpha^{\prime}}\right)+B_{l, m}^{\alpha^{\prime}, l o} \dot{u}_{l}^{\alpha^{\prime}}\left(r^{\prime}, E_{l}^{\alpha^{\prime}}\right)\right] Y_{l, m}\left(\check{r}^{\prime}\right)} & \text { Región I } \\
0 & \text { Región II } .
\end{array} .\right.
$$

En este caso se utiliza el mismo conjunto de energías que para las funciones aunque no es estrictamente necesario. Los coeficientes $A_{l, m}^{\alpha^{\prime}, l o}$ y $B_{l, m}^{\alpha^{\prime}, l o}$ se determinan normalizando las funciones de onda pidiendo que los orbitales locales sean nulos en el borde de las esferas de muffin-tin (pero no de pendiente cero). De esta manera garantizamos que las funciones APW y las de orbitales locales sean continuas en el borde de la esfera pero para ambas su primera derivada es discontinua.

\section{Funciones de base mixtas LAPW/ APW+lo:}

La razón por la cual el método LAPW+LO necesita un mayor número de funciones de base que el método APW+lo es que algunos (pocos) del total de estados del sistema 
son muy "costosos" (en términos de cálculo computacional) para tratarlos con el LAPW, dichos estados son:

- Estados de valencia $d$ of.

- Estados en átomos que tienen una esfera de muffin-tin mucho más pequeña que otras esferas en la celda unidad.

Por lo tanto resulta ventajoso tratar estos estados con el método APW+lo y usar el LAPW para el resto de los estados. Esto se debe a que para tratar un dado estado con APW+lo se necesita agregar $2 l+1$ orbitales locales por átomo a las funciones de la base. Esto hace que para el mismo $R_{K M A X}$ (el cual se define como el producto del radio de muffin-tin y el vector de onda más grande de la red recíproca) el tamaño de la base APW+lo sea considerablemente mayor que la base LAPW. Esta aparente dificultad en realidad se ve compensada porque para obtener resultados adecuados es necesario un $R_{\text {KMAX }}$ más pequeño en APW+lo respecto del utilizado en LAPW. No obstante se recomienda usar orbitales locales solo donde sea necesario usarlos, lo que conduce a usar funciones del tipo (4.25) para todo los átomos y para orbitales característicos de determinados átomos arbitrarios usar funciones del tipo (4.33), conformando así el total de las funciones una base mixta LAPW/APW+lo. Este tipo de base es lo que propone el código WIEN2K [Blaha, 1999].

\section{4-3 Metodología del cálculo FP-APW+lo para el caso de impurezas en semiconductores}

Para calcular con el método FP-LAPW las componentes del tensor GCE en los sitios de impureza de los óxidos estudiados en el presente trabajo, simulamos la dilución de la sonda en el sólido reemplazando un átomo-impureza por un catión en la celda a calcular.

En el caso de los óxidos con estructura bixbita, como veremos más adelante, poseen una celda unidad de 80 átomos (32 cationes y 48 aniones) y su parámetro de red es del orden de 10 Å. En estos sistemas se utilizó una dilución de 1:32 ( $\mathrm{N}^{\mathrm{o}}$ de impurezas: $\mathrm{N}^{\mathrm{o}}$ cationes), es decir que se reemplazó una impureza por un catión originario en la celda unidad. Con esta dilución veremos que las medidas experimentales son bien reproducidas.

En el caso de los otros óxidos bajo estudio, cuyos parámetros de red son mucho menores a los de las bixbitas, la dilución utilizada en cada caso fue determinada de 
modo de reproducir las medidas del tensor GCE experimentales. En general se construyeron superceldas de cálculo repitiendo la celda unidad en las direcciones $\boldsymbol{a}, \boldsymbol{b}$ y c de modo que la celda resultante tenga parámetros de red del orden de los 10 Å. Y en esta supercelda se reemplazó un catión del óxido puro por un átomo-impureza. Con esta dilución se comprobó que cada sonda-impureza PAC no percibe los efectos de su sonda vecina más próxima debido a la dependencia del GCE con $r^{-3}$, donde $r$ es la distancia desde el sitio donde se determina el GCE hasta la carga que lo produce.

Para considerar el estado de carga de la celda y de la impureza, se realizaron cálculos en función del estado de carga de la celda. En el caso de que la impureza se comporte como aceptora (valencia de la impureza menor que la del átomo originario) se realizan cálculos con la celda neutra (impureza en estado aceptor) y con celda cargada (agregando electrones, impureza ionizada). Mientras que si la sonda es de carácter donor (valencia de la impureza mayor que la del átomo originario) el caso cargado corresponde a remover electrones de la celda. En todos los casos de celda cargada se debió compensar el exceso de carga agregando un fondo positivo (carácter aceptor) o negativo (carácter donor) según corresponda, para mantener la neutralidad de carga de la celda. Para obtener los desplazamientos de los oxígenos primeros vecinos debido a las relajaciones estructurales producidas por la inclusión de la impureza en la red huésped, se calcularon las fuerzas resultantes sobre cada átomo de la celda y en función de estas, mediante un esquema de amortiguamiento de Newton [Kohler, 1996], se obtuvieron las posiciones de equilibrio. Este procedimiento se repitió hasta que la fuerza sobre cada ión fuese menor al valor de 0.025 eV/Å. Además, en cada paso se obtuvieron las componentes $\mathrm{V}_{\mathrm{ii}}$ del tensor GCE en el sitio de la impureza a partir de las componentes $\mathrm{V}_{2 \mathrm{M}}$ de la expansión armónica del potencial autoconsistente [Errico, 2002].

A modo de ejemplo de como analizar la composición del estado de impureza localizado en el nivel de Fermi, presentamos los resultados ya existentes obtenidos para el caso de $\mathrm{TiO}_{2}$ dopado con Cd [Errico, 2003]. Los cálculos se realizaron en una supercelda de 72 átomos, esto se esquematiza en la Figura 4.3. La Figura 4.4 muestra las densidades de estados parciales (PDOS) para los átomos de Cd, y los oxígenos primeros vecinos $\mathrm{O} 1$ y $\mathrm{O} 2$ para celda neutra (72A-SC(0)) y celda cargada (72A-SC(-2)) [Errico, 2003]. Cabe aclarar que el cálculo de celda neutra en este caso corresponde a reemplazar un átomo de $\mathrm{Ti}$ por un átomo de $\mathrm{Cd}$, mientras que en el cálculo de celda 
cargada se hace el mismo reemplazo y se agregan dos electrones debido a que el Cd actúa como doble aceptor en este sistema.

En la Figura 4.4 se ve claramente como aparecen niveles de impureza en las proximidades de la energía de Fermi $\left(\mathrm{E}_{\mathrm{f}}\right)$, con la diferencia que en el caso de la celda cargada, todos los niveles de impurezas quedan ocupados. Para el Cd, los niveles de impureza están relacionados con orbitales de simetría $\mathrm{d}_{\mathrm{yz}}$, esto muestra que los electrones que ionizan al Cd van a la ligadura Cd-O1. El llenado y vaciado de estos orbitales de una determinada simetría en el nivel de impurezas, justifica que el GCE cambie de módulo, signo y dirección al tratar un tipo de celda o la otra. Esto se muestra en la Tabla 4.1.

Notar que los parámetros de la supercelda $\left(a^{\prime}=b^{\prime}=9.169 \AA\right.$ y $c^{\prime}=8.859 \AA$ [Errico 2003]) son próximos a los $10 \AA$ y como puede corroborarse en la tabla con estas dimensiones (con esta dilución), la medida PAC del tensor GCE experimentado por la sonda Cd en el sitio catiónico del óxido de titanio está en un acuerdo aceptable con el cálculo de la celda cargada considerando las relajaciones introducidas por la inclusión de la impureza en la red huésped.

(a)

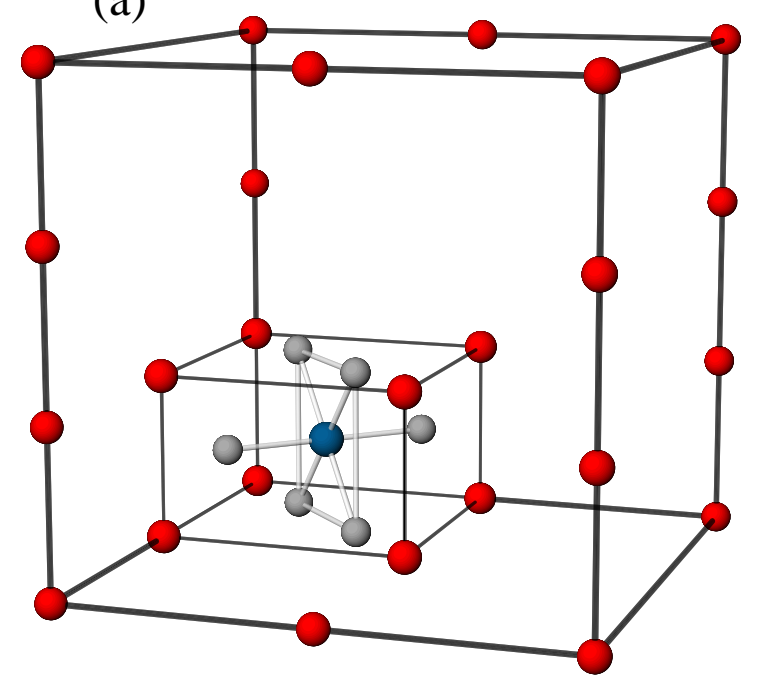

(b)

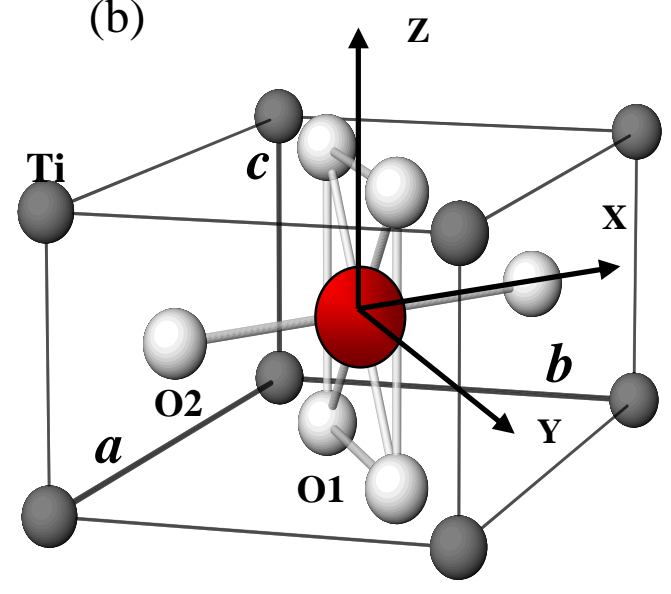

Figura 4.3: (a) Supercelda empleada en los cálculos de estructura electrónica de $\mathrm{TiO}_{2}$ :Cd $\left(a^{\prime}=2 a, b^{\prime}=2 b\right.$ y $\left.c^{\prime}=3 c\right)$. Para simplificar sólo se muestran los oxígenos primeros vecinos (esferas grises) a la impureza (esfera azul) y algunos de los Ti (esferas rojas). (b) celda unidad del $\mathrm{TiO}_{2}$ con su sistema de ejes cristalinos, sistemas de ejes principales X Y Z donde el GCE es diagonal y las constantes de red. 


\begin{tabular}{|c|c|c|c|c|c|}
\hline SONDA & \multicolumn{2}{|c|}{ Método } & $\boldsymbol{V}_{\mathbf{3 3}}\left[\mathbf{1 0}^{\mathbf{2 1}} \mathbf{V} / \mathbf{m}^{\mathbf{2}}\right]$ & $\boldsymbol{\eta}$ & Dirección de $\boldsymbol{V}_{\mathbf{3 3}}$ \\
\hline \multirow{4}{*}{$\boldsymbol{*} \boldsymbol{*} d$} & \multirow{2}{*}{$\begin{array}{c}\text { FP-LAPW sin } \\
\text { relajaciones [1] }\end{array}$} & Neutra & -12.84 & 0.54 & $\mathbf{X}$ \\
\cline { 2 - 6 } & $\begin{array}{c}\text { FP-LAPW con } \\
\text { relajaciones [1] }\end{array}$ & Neutra & -7.16 & 0.91 & $\mathbf{X}$ \\
\cline { 2 - 6 } & Cargada & +4.52 & 0.27 & $\mathbf{X}$ \\
\cline { 2 - 6 } & Experimental [2] & & $5.34(1)$ & $0.18(1)$ & $\mathbf{X ~ o ~ Y ~}$ \\
\hline
\end{tabular}

Tabla 4.1: Dirección, signo y magnitud de $V_{33}$ y $\eta$ obtenidos con el método de cálculo FP-LAPW (en la aproximación LDA) para el caso de la celda sin y con relajaciones.

[1] L.A. Errico, G. Fabricius, and M. Rentería, Phys. Rev. B 67, 144104 (2003).

[2] L.A. Errico, G. Fabricius, M. Rentería, P. de la Presa, and M. Forker, Phys. Rev. Lett. 8955503 (2002).
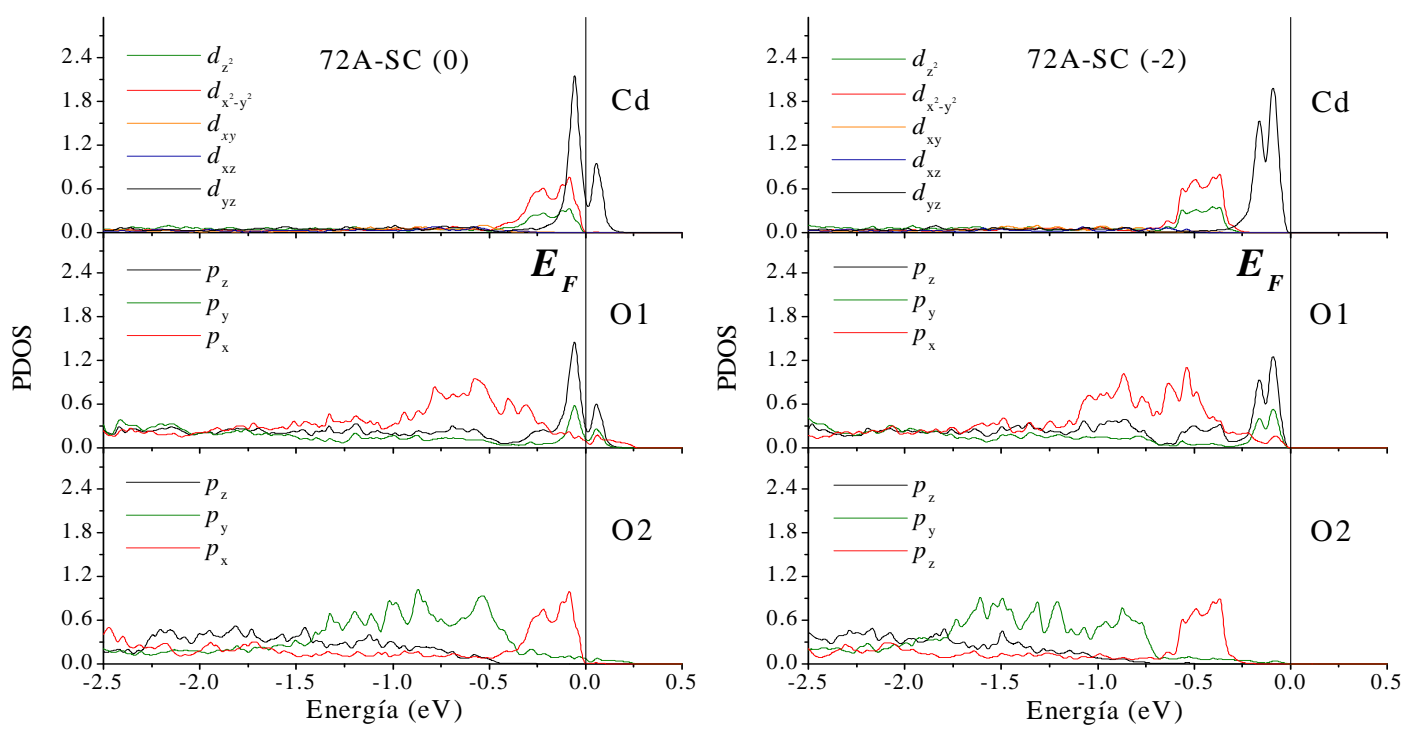

Figura 4.4: Densidades parciales de estados (PDOS) para $\mathrm{Cd}$, $\mathrm{O} 1$ y O2, en los sistemas 72A-SC(0) (izquierda) y 72A-SC(-2) (derecha). La energía se mide respecto del nivel de Fermi.

\section{Referencias}

[GITSUM 1988] GITSUM: código facilitado por J. Kesten, ZPI G“ottingen (1988).

[EIGEN 1962] EIGEN: subrutina basada en el método de diagonalización desarrollado por Jacobi y adaptado por Von Neumann para grandes ordenadores. Puede encontrarse en: Mathematical Methods for Digital Computers, John Wiley \& Sons, New York (1992). 
[Sternheimer 1953] R. M. Sternheimer y H. M. Foley, Phys. Rev. 92, 1460 (1953); R. M. Sternheimer y H. M. Foley, Phys. Rev. 102, 731 (1956); R. M. Sternheimer, Phys. Rev. 95, 736 (1954); R. M. Sternheimer, Phys. Rev. 96, 951 (1954); R. M. Sternheimer, Phys. Rev. 130, 1423 (1963); R. M. Sternheimer, Phys. Rev. 132, 1637 (1963); R. M. Sternheimer, Phys. Rev. 146, 140 (1966); R. M. Sternheimer, Phys. Rev. 159, 266 (1967).

[Feiock 1969] F. D. Feiock y F. W. Johnson, Phys. Rev. B 39, 187 (1969). El valor correspondiente al $\mathrm{Ta}^{5+}$ proviene de la interpolación del parámetro de $\mathrm{Hf}^{5+} \mathrm{y}^{5 e} \mathrm{~W}^{6+}$. [Thomas, 1926] L. H. Thomas, Proc. Camb. Phil. Soc. 23, 542 (1926).

[Fermi, 1928] E. Fermi, Zeit. fur. Physik 48, 73 (1928).

[Dirac, 1930] P. A. M. Dirac, Proc. Camb. Phil. Soc. 26, 376 (1930)

[Hohemberg, 1964] H. Hohemberg y W. Kohn, Phys. Rev. 136, B864 (1964).

[Perdew, 1992] J. P. Perdew y Y.Wang, Phys. Rev. B 45, 13244 (1992).

[Perdew, 1996] J. P. Perdew, K. Burke, M. Erzenhorf y Y.Wang, Phys. Rev. Lett. 77, 3865 (1996).

[Slater, 1937] J. C. Slater, Phys. Rev. 51, 846 (1937).

[Cottenier 2002] S. Cottenier Density Functional Theory and the Family of (L)APWmethods: a step-by-step introduction (2002).

[Blaha, 1988] P. Blaha, K. Schwarz y P. Herzig, Phys. Rev. B 37, 2792 (1988).

[Blaha, 1989] P. Blaha, P. I. Sorantin, C. Ambrosch y K. Schwarz, Hyp. Int. 51, 917 (1989).

[Weinert, 1981] M. Weinert, J. Math Phys. 22, 2433 (1981).

[Schwarz 1990] K. Schwarz, C. Ambrosch-Draxl y P. Blaha, Phys. Rev. B 42, 2051 (1990).

[Kohler, 1996] B. Kohler, S. Wilke, M.Scheffler, R. Kouba y C. Ambrosch-Draxl, Comp. Phys. Comm. 94, 31 (1996).

[Errico, 2002] L. A Errico, G. Fabricius y M. Rentería, P. De La Presa y M.Forker, Phy. Rev. Lett 89, 55503 (2002).

[Errico, 2003] L. A Errico, G. Fabricius y M. Rentería, Phy. Rev. B 67, 144104 (2003). 


\section{Capítulo N5}

\section{Sistemas estudiados}

En el presente trabajo se midieron óxidos binarios seleccionados con la sonda $\left({ }^{111} \mathrm{In} \rightarrow\right)^{111} \mathrm{Cd}$ con el fin de estudiar estudiar la dependencia de las interacciones hiperfinas dinámicas observadas en función de las propiedades estructurales y electrónicas de los sistemas huésped. Particularmente se midieron los óxidos con estructura Bixbita $\mathrm{Sc}_{2} \mathrm{O}_{3}, \mathrm{Y}_{2} \mathrm{O}_{3}$ e $\mathrm{In}_{2} \mathrm{O}_{3}$; el óxido $\mathrm{SnO}_{2}$ el cual presenta estructura Rutilo; el monóxido SnO cuya celda unidad presenta simetría tetragonal y el monóxido de zinc ( $\mathrm{ZnO})$ caracterizado por tener una celda unidad de simetría hexagonal.

Algunas de los óxidos con estructura bixbita estudiados en la literatura con la técnica PAC presentan interacciones dinámicas debidas a los efectos posteriores (after effects, AE) a la CE del ${ }^{111} \operatorname{In}\left(\rightarrow{ }^{111} \mathrm{Cd}\right)$. Tales bixbitas son aquellas cuyos cationes tienen capas electrónicas cerradas como ser el $\mathrm{Sc}_{2} \mathrm{O}_{3}, \mathrm{Y}_{2} \mathrm{O}_{3}$ (óxidos estudiados por Bartos et al. [Bartos 1991]) y $\mathrm{In}_{2} \mathrm{O}_{3}$ [Desimoni 1983, Bibiloni 1984, Habenicht 1996]. Sus espectros $R(t)$ observados a temperatura ambiente presentan apreciables amortiguamientos, los cuales desaparecen a temperaturas superiores a $800 \mathrm{~K}$. El amortiguamiento de los espectros PAC es reversible con la temperatura por lo que no puede ser atribuido a algún tipo de daño por radiación. Además, no puede ser descripto por una distribución estática de frecuencias. En estas medidas se observa una disminución del hard-core experimental $S_{20}$ (ver ecuación 2.27), la cual no puede ser descripta por una distribución estática del GCE. Este fenómeno está de acuerdo con efectos producidos por interacciones hiperfinas dinámicas. Por otro lado, existen en la literatura medidas PAC en óxidos de tierras raras con estructura bixbita, en las que no se han detectado interacciones dinámicas [Shitu 1998, Bartos 1991]. En estos óxidos, la configuración electrónica del catión es tal que tiene la capa $4 f$ incompleta, característica que ha sido correlacionada con una rápida ocupación de los huecos electrónicos producidos por la EC del ${ }^{111} \operatorname{In}\left(\rightarrow{ }^{111} \mathrm{Cd}\right)$ [Shitu 1995].

Por otro lado, experimentos PAC realizados en el óxido de estructura rutilo $\mathrm{SnO}_{2}$ medidos con $\left({ }^{111} \mathrm{In} \rightarrow\right){ }^{111} \mathrm{Cd}$ revelan características similares a las mencionadas en las 
bixbitas anteriores [Wolf 1986, Bibiloni 1988, Rentería 1991]. El amortiguamiento de los espectros PAC es apreciable desde los $17 \mathrm{~K}$ y recién desaparece cuando la temperatura de medida es mayor o igual a $900 \mathrm{~K}$.

Respecto al semiconductor SnO, existían medidas PAC con $\left({ }^{111} \mathrm{In} \rightarrow\right)^{111} \mathrm{Cd}$ pero el GCE resultó muy difícil de caracterizar, de hecho solo se pudo observar una interacción que aparece en un pequeño intervalo de los tratamientos térmicos, la cual fue atribuida tentativamente a sondas $\left({ }^{111} \mathrm{In} \rightarrow\right){ }^{111} \mathrm{Cd}$ localizadas substitucionalmente en el SnO [Rentería, 1991]. Pero debido a que los espectros PAC medidos a temperatura ambiente fueron muy amortiguados, no se pudo confirmar el valor $\eta=0.00$ predicho por la estructura cristalina. Con este antecedente realizamos nuevas medidas PAC en este sistema [Muñoz 2007], tales medidas muestran un claro amortiguamiento en los espectros en el rango de temperatura 300 - $600 \mathrm{~K}$, el cual se levanta parcialmente a muy bajas temperaturas $(10 \mathrm{~K}-100 \mathrm{~K})$ y totalmente a temperaturas altas (700 K - $1000 \mathrm{~K})$. Un resultado interesante de este estudio preliminar es que fueron caracterizadas dos interacciones hiperfinas (estáticas a altas temperaturas) estables en todo el rango de temperatura, a pesar de que la estructura cristalina del SnO presenta un solo sitio catiónico.

Finalmente, el monóxido de zinc $(\mathrm{ZnO})$ es estudiado en el presente trabajo porque presentaba aspectos particularmente interesantes. Por un lado, medidas PAC en $\mathrm{ZnO}:{ }^{111} \mathrm{Cd}$ realizadas en un amplio rango de temperaturas (77 K - $1075 \mathrm{~K}$ ) mostraban un débil amortiguamiento de los espectros. Por otro lado, medidas PAC en sondas $\left({ }^{111} \mathrm{In} \rightarrow\right){ }^{111} \mathrm{Cd}$ en $\mathrm{ZnO}$ dopado adicionalmente con impurezas Co muestran la aparente ausencia de interacciones dinámicas y la presencia de sólo una interacción hiperfina, es decir que el dopaje del $\mathrm{ZnO}$ con Co no produce nuevas interacciones hiperfinas [Marcio 2009]. Finalmente, medidas PAC en ZnO dopado con In y medido con la sonda $\left({ }^{111} \mathrm{In} \rightarrow\right)^{111} \mathrm{Cd}$ muestran la aparición de nuevas interacciones hiperfinas y un claro amortiguamiento en los espectros $\mathrm{R}(\mathrm{t})$ evidenciando interacciones dinámicas [Sato 2008]. Cabe destacar que el ión $\mathrm{Cd}^{2+}$ es isovalente respecto del ión $\mathrm{Zn}^{2+}$ en la red del $\mathrm{ZnO}$, lo que lo hace un sistema muy interesante para estudiar la presencia de interacciones dinámicas a la luz medidas PAC y cálculos ab initio del GCE en función del estado de carga de la celda. 


\section{5-1 Sesquióxidos con estructura bixbita: $\mathrm{Sc}_{2} \mathrm{O}_{3}, \mathrm{Y}_{2} \mathrm{O}_{3}$ e $\operatorname{In}_{2} \mathrm{O}_{3}$}

Los sesquióxidos abordados en la presente tesis cristalizan en la estructura cúbica de la bixbita $\left(\mathrm{X}_{2} \mathrm{O}_{3}\right.$ con $\mathrm{X}=\mathrm{Sc}$, In, e $\left.\mathrm{Y}\right)$. A diferencia de los óxidos de tierras raras con estructura bixbita (C- $\mathrm{X}_{2} \mathrm{O}_{3}$ con $\mathrm{X}=\mathrm{Lu}, \mathrm{Yb}, \mathrm{Tm}, \mathrm{Er}, \mathrm{Ho}, \mathrm{Dy}, \mathrm{Gd}$, Eu y Sm), estos óxidos poseen cationes con capas electrónicas cerradas. Además de su alta estabilidad térmica, los óxidos de Sc, Y, e In no presentan polimorfismo, a diferencia de las bixbitas de tierras raras, lo que hace que la bixbita sea la única fase estable en todo el rango de temperatura.

En la estructura bixbita, los cationes forman una red cúbica centrada en las caras en la cual seis de los ocho sitios tetraédricos están ocupados por oxígenos. La celda unidad de grupo espacial $T_{H}{ }^{7}\left(\mathrm{Ia}^{3}\right)$ consiste de ocho de estos cubos conteniendo 32 cationes y 48 oxígenos (ver Figura 5.1 (a)). La estructura presenta dos sitios cristalográficos catiónicos inequivalentes, denominados C y D, y uno para los oxígenos. La abundancia relativa de los sitios $\mathrm{C}$ y D en la red ideal es 3:1, respectivamente. En ambos sitios los cationes tienen una coordinación $\mathrm{NN}=6$ ( $\mathrm{NN}$ por nearest neihgbors, primeros vecinos). Mientras que los oxígenos poseen una coordinación $\mathrm{NN}=4$. El sitio D es axialmente simétrico y puede describirse a partir de un catión rodeado por seis átomos de oxígeno que ocupan los vértices de un cubo deformado, dejando libres dos vértices sobre una misma diagonal de dicho cubo (ver Figura 5.1 (b), inferior). El sitio C presenta una coordinación con los átomos oxígeno NN más irregular por lo que el cubo se vuelve más distorsionado (ver Figura 5.1 (b), superior). Se puede pensar la coordinación de este sitio en forma similar a la anterior con la diferencia que ahora quedan libres dos vértices sobre la diagonal de una de las caras del cubo [Marezio 1966, Eyring 1979]. En cuanto a la coordinación de segundo vecinos (NNN por next nearest neighbors), el sitio C posee 12 cationes NNN, de los cuales 8 son sitios $C$ y 4 son sitios $D$, mientras que el sitio D presenta 12 cationes NNN, todos del tipo C. 


\begin{tabular}{ccccccc}
\hline \hline Óxido & $\boldsymbol{a}[\AA]$ & $-\boldsymbol{u}$ & $\boldsymbol{x}$ & $\boldsymbol{y}$ & $\boldsymbol{z}$ & Referencia \\
\hline $\mathrm{In}_{2} \mathrm{O}_{3}$ & $10.117(1)$ & $0.0332(1)$ & $0.3905(13)$ & $0.1529(11)$ & $0.3832(12)$ & [Marezio 1966] \\
\hline $\mathrm{Y}_{2} \mathrm{O}_{3}$ & $10.5989(7)$ & $0.03236(3)$ & $0.3907(2)$ & $0.1518(2)$ & $0.3801(2)$ & [Maslen 1996] \\
\hline $\mathrm{Sc}_{2} \mathrm{O}_{3}$ & 9.845 & 0.03546 & 0.39137 & 0.15477 & 0.38137 & $\begin{array}{c}\text { [Wyckoff 1964, } \\
\text { Norrestam 1968] }\end{array}$ \\
\hline \hline
\end{tabular}

Tabla 5.2: Prámetros cristalográficos de los sesquióxidos de In, Y, y Sc. Para todos los casos $\alpha=\beta=\gamma=90^{\circ}$.

\section{5-2 El dióxido de Estaño $\left(\mathrm{SnO}_{2}\right)$}

El óxido $\mathrm{SnO}_{2}$ cristaliza en la misma estructura que el $\mathrm{TiO}_{2}$, la cual lleva el nombre de rutilo. La estructura rutilo presenta un solo sitio de catión coordinado con seis oxígenos primeros vecinos. Los seis oxígenos forman un octaedro distorsionado centrado en el catión $\mathrm{Sn}^{+4}$, cuatro de ellos a la misma distancia del Sn (2.007 А⿱) y formando el plano basal rectangular del octaedro, los otros dos oxígenos se sitúan en los vértices del octaedro ambos a la misma distancia del catión (2.017 Å). La celda unidad y la configuración del catión con sus primeros vecinos se muestran en la Figura 5.2 (a). Cabe destacar que si bien la estructura rutilo posee un solo sitio inequivalente de catión, el octaedro de primeros vecinos presenta dos posibles orientaciones en la red que difieren en una rotación de $90^{\circ}$ alrededor del eje $c$. Esto se muestra en la Figura 5.2 (b). La celda unidad del $\mathrm{SnO}_{2}$ es tetragonal y posee dos moléculas (6 átomos). El grupo espacial de esta estructura es $D_{4 h}{ }^{14}(P 4 / \mathrm{mnm})$. Los átomos metálicos se encuentran en posiciones de tipo $2 a$ localizadas en $\left(\begin{array}{llll}0 & 0 & 0\end{array}\right)$ y $\left(1 / 2 \frac{1}{2} \frac{1}{2} / 2\right)$, y las posiciones de los aniones son del tipo $4 f$ situadas en $\sharp\left(u \quad u \quad 0, u+1 / 2 \frac{1}{1 / 2}-u\right.$ 1/2) con $u=0.3056(1)$ [Bolzan 1997]. Los parámetros de red del rutilo $\mathrm{SnO}_{2}$ son $a=b=4.7374(1) \AA, c=3.1864 \AA$ y $\alpha=\beta=\gamma=90^{\circ}$ [Bolzan 1997]. 
(a)

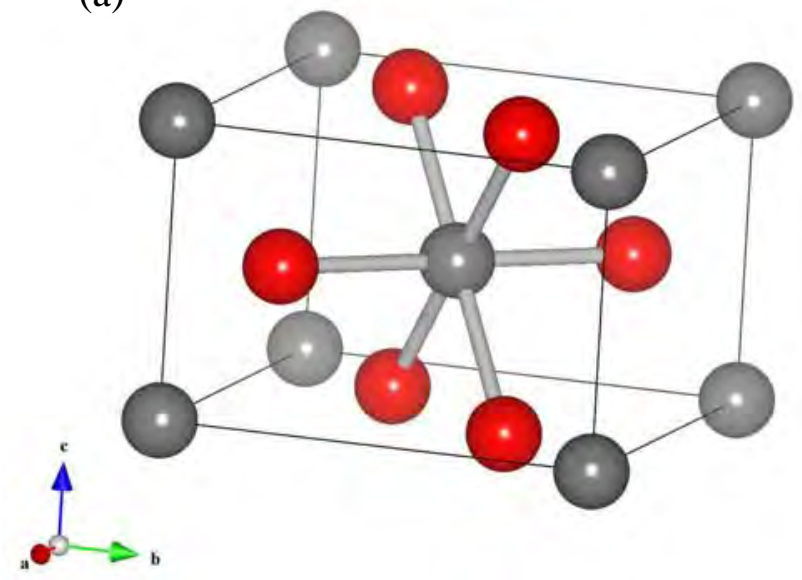

Figura 5.2: (a) Celda unidad del $\mathrm{SnO}_{2}$ con estructura rutilo donde se muestra con gris el $\mathrm{Sn}$ con su coordinación $\mathrm{NN}=6$. Los oxígenos están representados con esferas rojas y los otros Sn de la celda unidad también con esferas grises. (b) Orientaciones de los octaedros en la estructura rutilo rotados $90^{\circ}$ entre si. (b)
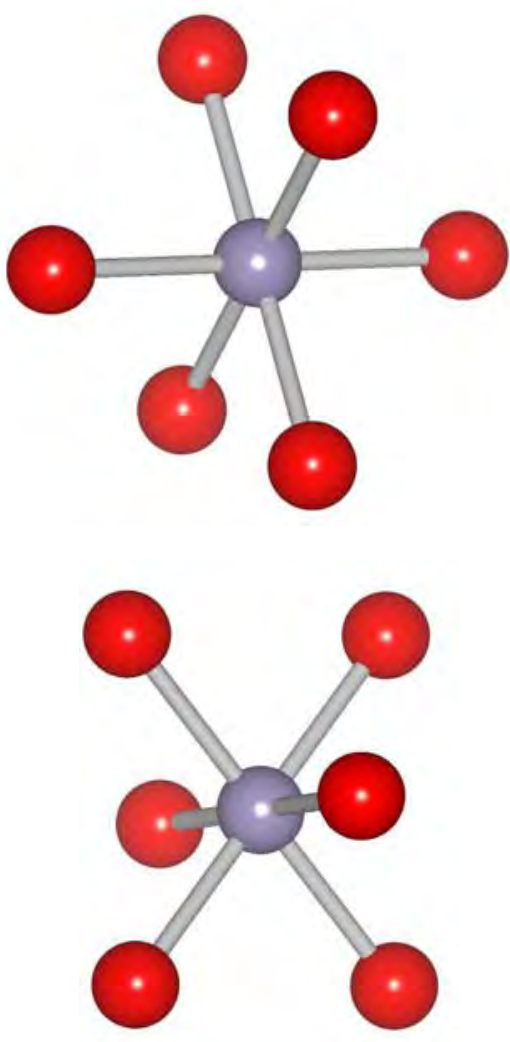

\section{5-3 El monóxido de Estaño (SnO)}

La coordinación del único sitio del catión $\mathrm{Sn}^{+2}$ en la estructura del $\mathrm{SnO}$ se realiza con un arreglo cuadrado de cuatro oxígenos pero con el átomo de Sn desplazado perpendicularmente de su centro como se muestra en la Figura 5.3 (a). Por el tipo de coordinación que tiene el catión con sus oxígenos NN (ver Figura 5.3 (b)) se lo suele incluir en el grupo de la tenorita, $\mathrm{CuO}$ (en este óxido los cuatro oxígenos NN están dispuestos en los vértices de un cuadrado y el $\mathrm{Cu}$ en el centro del mismo). La celda tetragonal que contiene dos moléculas del óxido (4 átomos) pertenece al grupo espacial $D_{4 h}{ }^{7}(P 4 / n m m)$. Las posiciones de los cationes son del tipo $2 c$ situadas en $\left(0 \frac{1}{2} u, 1 / 20-\right.$ u) con $u=0.2369$, mientras que las posiciones de los oxígenos son del tipo $2 a$ localizadas en $\left(\begin{array}{llllll}0 & 0 & 0,1 / 2 & 1 / 2 & 0\end{array}\right)$ [Wang 2004]. Los parámetros de red del SnO son $a=b=3.8029(5) \AA, c=4.8382(8) \AA$ y $\alpha=\beta=\gamma=90^{\circ}$ [Wang 2004]. 


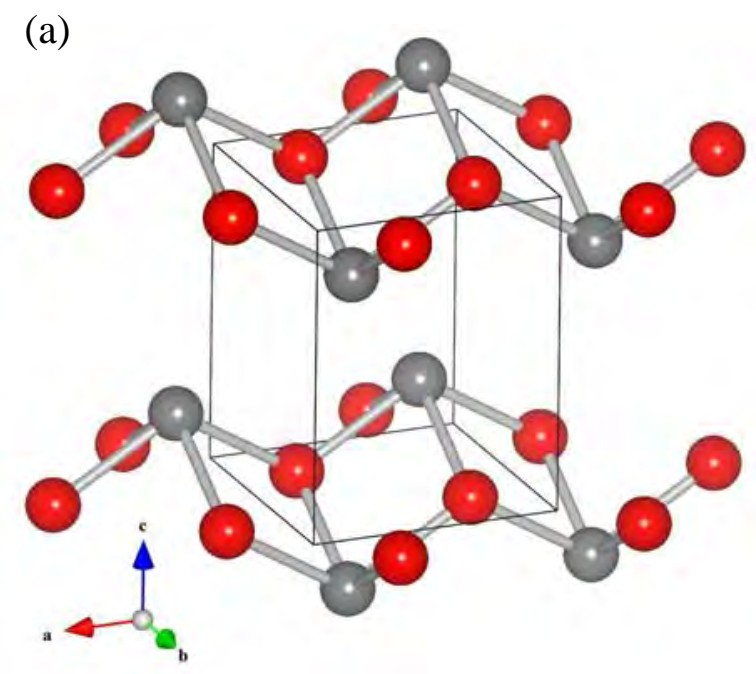

(b)

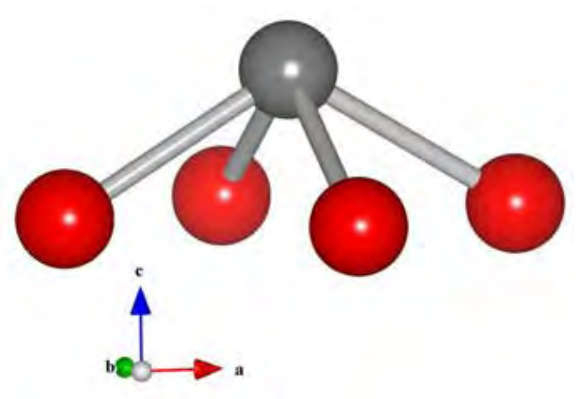

Figura 5.3: (a) Posiciones atómicas en la celda unidad del SnO, las esferas azules representan los átomos Sn y las esferas grises los átomos O. (b) Coordinación NN=4 del Sn.

\section{5-4 El monóxido de Zinc (ZnO)}

La celda hexagonal del $\mathrm{ZnO}\left(\alpha=\beta=90^{\circ}\right.$ y $\left.\gamma=120^{\circ}\right)$ contiene dos moléculas del óxido (cuatro átomos) tal como se muestra en la Figura 5.4 (a). Las posiciones atómicas de los cationes $\mathrm{Zn}^{+2}$ son $\left(\begin{array}{lll}0 & 0 & 0\end{array}\right)$ y $\left(\begin{array}{lll}1 / 3 & 2 / 3 & 1 / 2\end{array}\right)$, y los aniones $\mathrm{O}^{-2}$ se encuentran en $\left(\begin{array}{lll}0 & 0 & u\end{array}\right)$ y $(1 / 32 / 3 u+1 / 2)$ con $u=0.3817(3)$. Los parámetros de red que caracterizan las dimensiones de la celda son $a=b=3.2501 \AA$ y $c=5.2071(1) \AA$ [Kisi 1989]. La estructura cristalina del $\mathrm{ZnO}$ posee un solo sitio catiónico donde el $\mathrm{Zn}$ se encuentra coordinado con cuatro átomos oxígeno primeros vecinos dispuestos en los vértices de un tetraedro ligeramente alargado en la dirección del eje $c$ (ver Figura 5.3 (b)). 

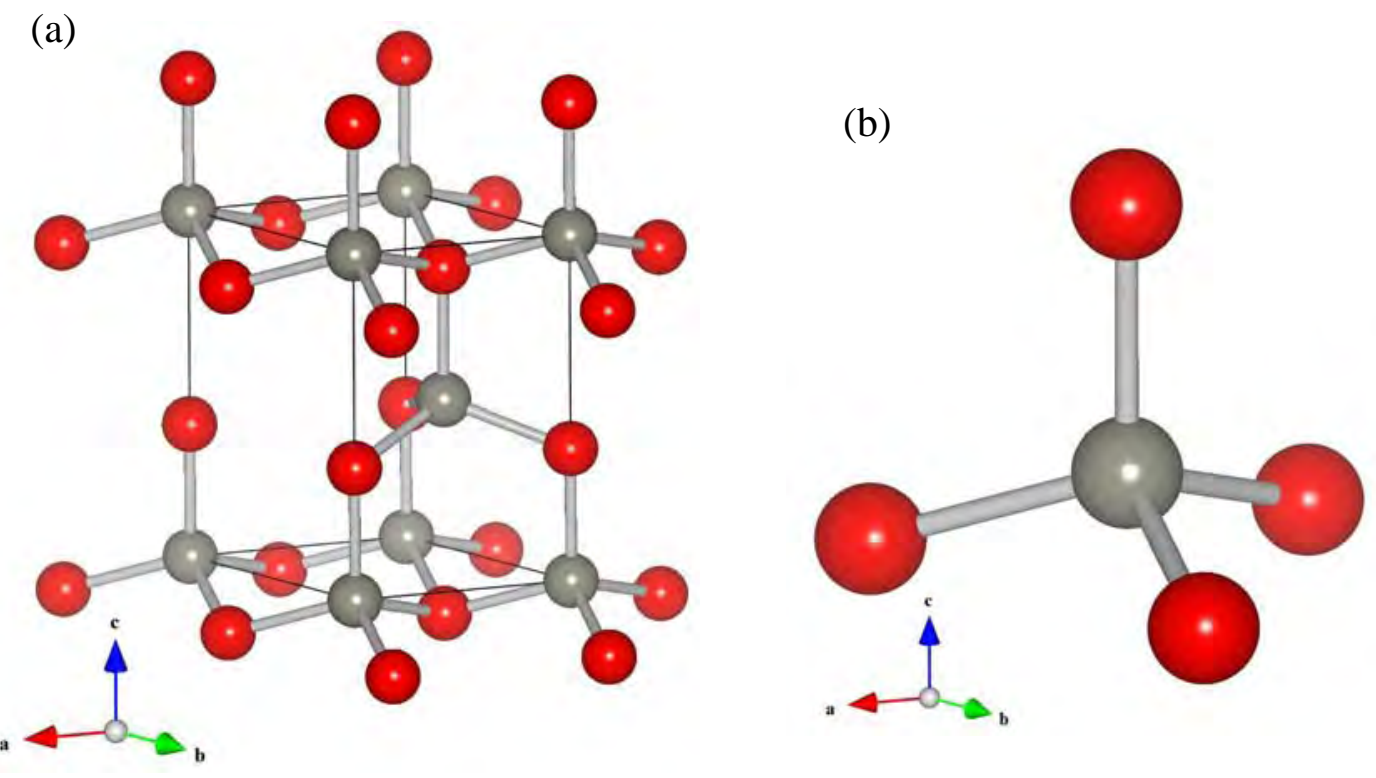

Figura 5.3: (a) Posiciones atómicas en la celda unidad del ZnO. Con esferas grises se representan los átomos Zn y con esferas rojas los átomos O. Las ligaduras se extienden a los primeros vecinos $(\mathrm{NN}=4)$. (b) Coordinación NN=4 del Zn.

\section{5-5 Preparación de las muestras}

Existen diferentes técnicas para introducir los atomos-sondas PAC en las muestras a ser medidas. Entre ellos podemos citar el dopaje por implantación iónica, por irradiación con neutrones térmicos, la inclusión de las impurezas por difusión térmica y la reacción en fase sólida asistida por trabajo mecánico. Para el caso de los experimentos realizados en esta Tesis, hemos utilizado la difusión térmica para la inclusión de las sondas en los respectivos sistemas. Describiremos rápidamente los métodos anteriormente nombrados para luego explicar detalladamente el dopaje de las muestras utilizadas en el presente trabajo.

Podemos distinguir entre dos clases de métodos para la inclusión de las sondas: en el primero, la sonda es producida o introducida en el óxido (por ejemplo, implantación iónica, irradiación con neutrones y difusión térmica); en el segundo tipo, la sonda es introducida durante la preparación del óxido (por ejemplo la reacción en fase sólida).

Implantación iónica: en este método la actividad se deposita en forma de cloruros en la fuente de iones, los que son generados por evaporación térmica de la solución y a 
posteriori, son acelerados e implantados en el blanco (muestra). Esta técnica ha demostrado ser uno de los métodos más efectivos para introducir la sonda en óxidos ya preparados pero tiene la desventaja de generar daños por radiación en la matriz del sólido. Este daño puede cambiar la homogeneidad de la muestra y hasta su estequiometría. En general, es removido por medio de tratamientos térmicos adecuados de unas pocas horas de duración.

Irradiación con neutrones térmicos: este método consiste en la producción de la sonda radiactiva a partir de reacciones nucleares en uno de los elementos del material a estudiar. Por ejemplo, cuando se utiliza la sonda $\left({ }^{181} \mathrm{Hf} \rightarrow\right)^{181}$ Ta se usa la reacción ${ }^{180} \mathrm{Hf}(\mathrm{n}, \gamma){ }^{181} \mathrm{Hf}$ para obtener el isótopo padre. Cabe destacar que la irradiación en nuestro país puede realizarse en el reactor RA-3 de la Comisión de Energía Atómica (CNEA) ubicado en Ezeiza, provincia de Buenos Aires, lo que facilita su aplicabilidad.

Reacción en fase sólida asistida por trabajo mecánico: En este método alternativo debe estudiarse su aplicación en cada caso particular debido a que no es de uso frecuente. Su aplicación correcta para el dopaje de muestras con ${ }^{181} \mathrm{Hf}$ en el futuro podría independizarnos de la disponibilidad de un implantador de iones. En general, se tiene polvo de $m-\mathrm{HfO}_{2}$ irradiado con neutrones termicos, tal como se explicó anteriormente, y polvo del óxido a estudiar (por ejemplo $\mathrm{X}_{\mathrm{a}} \mathrm{O}_{\mathrm{b}}$ ). Se realiza un mezclado manual de los mismos y se someten a un tratamiento térmico. Las proporciones de $\mathrm{HfO}_{2}$ y de $\mathrm{X}_{\mathrm{a}} \mathrm{O}_{\mathrm{b}}$ son elegidas de modo que $N^{H f} / N^{X} \approx 1 \%$ o menor ( $N^{H f}$ es el número de átomos Hf y $N^{X}$ es el número de átomos del elemento X), de modo que el isótopo ${ }^{181} \mathrm{Hf}$ sea una impureza del óxido $\mathrm{X}_{\mathrm{a}} \mathrm{O}_{\mathrm{b}}$. Luego del tratamiento térmico la muestra es sometida a molido mecánico. A posteriori, se realizan diferentes tratamientos térmicos y entre cada uno de ellos, se toman medidas PAC para estudiar el efecto de cada variable (molienda, temperatura, tiempo de tratamiento, etc.) sobre el sistema. Este método ha sido aplicado satisfactoriamente en el sesquióxido $\mathrm{Tm}_{2} \mathrm{O}_{3}$ [Muñoz 2009].

Difusión térmica: las muestras medidas en el marco del presente trabajo fueron preparadas vía esta metodología de dopaje. A pesar de que la eficacia de la actividad introducida en el sistema depende del compuesto a estudiar, esta técnica presenta la ventaja de no producir daños en la red cristalina. 


\begin{tabular}{|c|c|}
\hline Óxido & Proceso de difusión \\
\hline $\begin{array}{l}\mathrm{Sc}_{2} \mathrm{O}_{3} \\
\text { (Sigma-Aldrich } \\
99.999 \%)\end{array}$ & $\begin{array}{l}\text { - Sube la temperatura hasta } 150^{\circ} \mathrm{C} \text { con una tasa de crecimiento de } \\
5^{\circ} / \text { min la cual permanece constante durante } 1 \mathrm{~h} \text {. } \\
\text { - Sube con una tasa de } 3^{\circ} / \mathrm{min} \text { hasta } 500^{\circ} \mathrm{C} \text {. Permanece constante a } \\
500^{\circ} \mathrm{C} \text { durante } 10 \mathrm{hs} \text {. } \\
\text { - Sube con una tasa de } 2^{\circ} / \mathrm{min} \text { hasta } 800^{\circ} \mathrm{C} \text { y permanece constante a } \\
800^{\circ} \mathrm{C} \text { durante } 10 \mathrm{hs} \text {. } \\
\text { - Baja la temperatura hasta } 20^{\circ} \mathrm{C} \text {. }\end{array}$ \\
\hline $\begin{array}{l}\mathrm{In}_{2} \mathrm{O}_{3} \\
\text { (Sigma-Aldrich } \\
99.999 \%)\end{array}$ & $\begin{array}{l}\text { - Sube la temperatura hasta } 150^{\circ} \mathrm{C} \text { con una tasa de } 5^{\circ} / \text { min. Permanece } \\
\text { constante a } 150^{\circ} \mathrm{C} \text { durante } 1 \mathrm{~h} \text {. } \\
\text { - Sube hasta } 500^{\circ} \mathrm{C} \text { con una tasa de } 3^{\circ} / \mathrm{min} \text {. Permanece constante a } \\
500^{\circ} \mathrm{C} \text { durante } 6 \text { hs. } \\
\text { - Sube hasta } 800^{\circ} \mathrm{C} \text { con una tasa de } 2^{\circ} / \text { min. Permanece constante a } \\
800^{\circ} \mathrm{C} \text { durante } 10 \mathrm{hs} \text {. } \\
\text { - Baja la temperatura hasta } 20^{\circ} \mathrm{C} \text {. }\end{array}$ \\
\hline $\begin{array}{l}\mathrm{Y}_{2} \mathrm{O}_{3} \\
\text { (Sigma-Aldrich } \\
99.99 \%)\end{array}$ & $\begin{array}{l}\text { - Sube la temperatura con una tasa de } 5^{\circ} / \text { min hasta } 150^{\circ} \mathrm{C} \text {, permanece } \\
\text { constante durante } 1 \mathrm{~h} \text {. } \\
\text { - Sube hasta } 500^{\circ} \mathrm{C} \text { con una tasa de } 3^{\circ} / \mathrm{min} \text {. Permanece constante a } \\
500^{\circ} \mathrm{C} \text { durante } 6 \text { hs. } \\
\text { - Sube hasta } 800^{\circ} \mathrm{C} \text { con una tasa de } 2^{\circ} / \text { min y permanece constante a } \\
800^{\circ} \mathrm{C} \text { durante } 12 \mathrm{hs} \text {. } \\
\text { - Baja la temperatura hasta } 20^{\circ} \mathrm{C} \text {. }\end{array}$ \\
\hline $\begin{array}{l}\mathrm{SnO}_{2} \\
\text { (Sigma-Aldrich } \\
\geq 99.99 \% \text { ) }\end{array}$ & $\begin{array}{l}\text { - Sube la temperatura hasta } 150^{\circ} \mathrm{C} \text { con una tasa de } 5^{\circ} / \text { min. Permanece } \\
\text { constante a } 150^{\circ} \mathrm{C} \text { durante } 1 \mathrm{~h} \text {. } \\
\text { - Sube hasta } 500^{\circ} \mathrm{C} \text { con una tasa de } 3^{\circ} / \mathrm{min} \text {. Permanece constante a } \\
500^{\circ} \mathrm{C} \text { durante } 6 \mathrm{hs} \text {. } \\
\text { - Sube hasta } 800^{\circ} \mathrm{C} \text { con una tasa de } 2^{\circ} / \mathrm{min} \text {. Permanece constante a } \\
800^{\circ} \mathrm{C} \text { durante } 10 \mathrm{hs} \text {. } \\
\text { - Baja la temperatura hasta } 20^{\circ} \mathrm{C} \text {. }\end{array}$ \\
\hline \multirow{2}{*}{$\begin{array}{l}\text { SnO } \\
\text { (Sigma-Aldrich } \\
99.999 \%)\end{array}$} & $\begin{array}{l}1^{\circ} \text { experimento: } \\
\text { La sonda radiactiva fue difundida en vacío desde } 423 \mathrm{~K} \text { hasta } 1000 \mathrm{~K} \text {. Se } \\
\text { calentó a } 423 \mathrm{~K} \text { durante } 1.5 \text { h; } 525 \mathrm{~K}, 2.25 \text { h; } 700 \mathrm{~K}, 12 \text { h; } 800 \mathrm{~K}, 1.5 \text { h; } 900 \\
\mathrm{~K}, 2 \text { h; } 950 \mathrm{~K} \text {, 3h y } 1000 \mathrm{~K}, 15.5 \mathrm{~h} .\end{array}$ \\
\hline & $\begin{array}{l}2^{\circ} \text { experimento: } \\
\text { se utilizó una porción de la pastilla preparada en el primer } \\
\text { experimento. En el proceso de difusión la temperatura de la muestra } \\
\text { fue incrementándose suavemente hasta alcanzar los } 900 \mathrm{~K} \text { y se } \\
\text { mantuvo en esta temperatura durante } 4 \text { horas }\end{array}$ \\
\hline $\begin{array}{l}\mathrm{ZnO} \\
\text { (Sigma-Aldrich } \\
99.999 \%)\end{array}$ & $\begin{array}{l}\text { La sonda radiactiva fue difundida en una atmósfera de vacío ( } 10^{-3} \text { torr) } \\
\text { en pasos de temperatura desde } 423 \text { hasta } 1000 \mathrm{~K} \text {. La difusión fue } \\
\text { monitoreada con la técnica PAC. }\end{array}$ \\
\hline
\end{tabular}

Tabla 5.2: Características de los procesos difusión de cada óxido medido. 
La difusión de la sonda ${ }^{111}$ In ha sido aplicada con éxito en óxidos con estructura bixbita [Errico 2005] y en el rutilo $\mathrm{SnO}_{2}$ [Wolf 1986]. Además, el grupo de Interacciones Hiperfinas del IPEN (San Pablo, Brasil) a estudiado ampliamente la difusión térmica de ${ }^{111}$ In en $\mathrm{ZnO}$ [Mercurio 2007], mientras que en el marco de esta tesis se ha extendido su aplicabilidad al SnO [Muñoz 2007].

El proceso comienza diluyendo ${ }^{111} \mathrm{InCl}_{3}$ de procedencia comercial en una solución 0.05 normal de $\mathrm{HCl}$ en agua. Las pastillas (de $5 \mathrm{~mm}$ de diámetro) de los distintos óxidos estudiados fueron preparadas en una prensa hidráulica con una presión de $3 * 10^{8} \mathrm{~Pa}$, antes de gotearlas son sometidas a un tratamiento térmico a alta temperatura para un mejor sinterizado. Luego se gotea con la solución una pequeña porción de pastilla policristalina del óxido a medir. Después, la pastilla es secada en una lámpara infrarroja y colocada en un tubo de cuarzo. Posteriormente, el tubo es cerrado en una atmósfera de $\mathrm{N}_{2}$ (excepto la pastilla de $\mathrm{ZnO}$ que se coloca en una atmósfera de vacío). Finalmente, se realiza el proceso de difusión (en la Tabla 5.3 se detallan los tratamientos térmicos realizados sobre cada óxido).

\section{Referencias}

[Desimoni 1983] J. Desimoni, A.G. Bibiloni, L.A. Mendoza-Zélis, A.F. Pasquevich, F.H. Sanchez y A. Lopez-García, Phys. Rev. B 28, 5739 (1983).

[Bibiloni 1984] A.G. Bibiloni, J. Desimoni, C.P. Massolo, L.A. Mendoza-Zelis, A.F. Pasquevich, F.H. Sanchez y A. Lopez-García, Phys. Rev. B 29, 1109 (1984).

[Habenicht 1996] S. Habenicht, D. Lulascu, M. Uhrmacher, L. Ziegeler. K.P. Lieb y ISOLDE collaboration, Z. Phys. B 101, 187 (1996).

[Shitu 1998] J. Shitu, A.F. Pasquevich, A.G. Bibiloni, M. Rentería and F.G. Requejo, Modern Physics Letters B 12, 281 (1998).

[Bartos 1991] A. Bartos, K.P. Lieb, A.F. Pasquevich, M. Uhrmacher e ISOLDE collaboration, Phys. Lett. A 157, 513 (1991).

[Shitu 1995] J. Shitu, Tesis Doctoral, Biblioteca del Departamento de Física, Facultad de Ciencias Exactas, Universidad Nacional de La Plata (1992).

[Wolf 1986] Wolf, H., Deubler, S., Forkel, D., Foettinger, H., Iwatschenko-Borho, M., Meyer, M., Renn, M., Witthuhn, W., Mat. Sci. Forum. 10-12, 863-868 (1986).

[Bibiloni 1988] Bibiloni, A.G., Desimoni, J., Massolo, C.P., Rentería, M. Phys. Rev. B 38, 20-25 (1988). 
[Rentería 1991] M. Rentería, A.G. Bibiloni, M.S. Moreno, J. Desimoni, R.C. Mercader, A. Bartos, M. Uhrmacher and K.P. Lieb, J. Phys. - Condens. Matter 3, 3625 (1991).

[Muñoz 2007] E. L. Muñoz, A. W. Carbonari, L. A. Errico, A. G. Bibiloni, H. M. Petrilli, M. Rentería, Hyp. Int. 17837 (2007).

[Mercurio 2009] M. E. Mercurio, Investigação de interações hiperfinas em ZnO e Zn $\mathrm{n}_{(1-}$ ${ }_{x)} \mathrm{Co}_{\mathrm{x}}$ ) pela tecnica de correlação angular gama-gama perturbada, dissertação de mestrado, IPEN-USP, São Paulo, 2009.

[Sato 2008] W. Sato, Y. Itsuki, S. Morimoto, H. Susuki, S. Nasu, A. Shinohara, and Y. Ohkubo, Phys. Rev. B 78045319 (2008).

[Marezio 1966] M. Marezio, Acta Cryst. 20, 723 (1966).

[Eyring 1979] L. Eyring “Handbook on the Physics and Chemistry of Rare Earths”, Cap. 27, editado por K.A. Gschneidner Jr. y L. Eyring, North Hlland Publishing Co., Amsterdam (1979).

[Wyckoff 1964] R.W.G. Wyckoff, “Crystal Structures” editado por Interscience Publishers, vol. 1 (John Wiley \& Sons, New York, 1964).

[Maslen 1996] N. Maslen, V.A. Streltsov and N. Ishisawa, Acta Cryst. B 52, 414 (1996).

[Norrestam 1968] R. Norrestam, Ark. Kemi. 29, 343 (1968).

[Baltruschy 1984] R. Baltruschy and H. Brandy, Acta Cryst. B 40, 76 (1984).

[Wang 2004] X. Wang, F.X. Zhang, I Loa, K. Syassen, M. Hanfland, Y.L. Mathins, Phys. Status Solidi B 241, 3168 (2004).

[Bolzan 1997] A. Bolzan, C. Frong, B. Kennedy and C. Howard, Acta Cryst. B 53, 373 (1997).

[Kisi 1989] E.H. Kisi and M.M. Elcombe, Acta Cryst. C 45, 1867 (1989).

[Muñoz 2009] E.L. Muñoz, G.N. Darriba, A.G. Bibiloni, L.A. Errico, M. Rentería, Journal of Alloys and Compounds 495, 532 (2010).

[Errico 2005] L.A. Errico, M. Rentería, A.G. Bibiloni, G.N. Darriba, Phys. Stat. Sol. (c) 10, 3576 (2005).

[Mercurio 2007] M.E. Mercurio, A.W. Carbonari, M.R. Cordeiro, and R.N. Saxena, Hyperfine Interact 178, 247 (2007). 


\section{Capítulo N6}

\section{Resultados experimentales}

\section{6-1 Resultados PAC del óxido SnO medido con la sonda $\left({ }^{111} \mathrm{In} \rightarrow\right){ }^{111} \mathrm{Cd}$}

Dentro del abordaje experimental del presente trabajo de Tesis se realizaron experimentos PAC con los óxidos descriptos en el capítulo anterior. Cabe destacar que los parámetros hiperfinos que caracterizan al GCE, obtenidos vía el mejor ajuste realizado con el modelo de Baverstäm y Othaz, corresponden al estado final estable alcanzado por la sonda ${ }^{111} \mathrm{Cd}$ luego del proceso dinámico experimentado a una temperatura dada. De este modo, la dependencia del GCE en función de la temperatura mostrada para cada óxido corresponde al GCE final estable observado por la sonda ${ }^{111}$ Cd luego del proceso dinámico.

Se eligió en primera instancia el óxido $\mathrm{SnO}$ puesto que presenta un interesante escenario para estudiar la presencia de interacciones dinámicas en $\mathrm{SnO}$ dopado con la sonda ${ }^{111} \mathrm{Cd}$ puesto que el $\mathrm{Cd}^{2+}$ y el $\mathrm{Sn}^{2+}$ son isovalentes en la estructura del $\mathrm{SnO}$ y además ambos iones son de capa cerrada. En este sistema se realizaron dos experimentos. En el primero los espectros $\mathrm{R}(\mathrm{t})$ fueron medidos en el rango de temperaturas 77 - $900 \mathrm{~K}$ pero entre $77 \mathrm{~K}$ y $295 \mathrm{~K}$ no se realizaron medidas. En el segundo experimento, se midió en el rango 20 - $900 \mathrm{~K}$ y particularmente se tomaron muchos puntos entre 20 y $300 \mathrm{~K}$. A continuación se presentan los resultados PAC del primer experimento del sistema $\mathrm{SnO}$ dopado con ${ }^{111} \mathrm{Cd}$. Cabe destacar que los resultados del primer experimento han sido publicados en [Muñoz 2007].

\section{6-1-1 Resultados PAC del primer experimento en SnO: $\left({ }^{111} \mathrm{In} \rightarrow\right){ }^{111} \mathrm{Cd}$}

En la Figura 6.1 (izquierda) se grafican los espectros $\mathrm{R}(\mathrm{t})$ y sus respectivas transformadas de Fourier para algunas temperaturas características en el rango 77 - 900 K. En los espectros $\mathrm{R}(\mathrm{t})$ tomados a altas temperaturas se aprecia que coexisten 2 interacciones hiperfinas estáticas. Por debajo de $700 \mathrm{~K}$, la señal de los espectros se atenúa apreciablemente, mientras que a $77 \mathrm{~K}$ la señal se recupera parcialmente. El hecho que este proceso sea reversible con la temperatura y que el factor $\mathrm{S}_{20}$ disminuya a 
temperaturas intermedias hace evidente la presencia de una interacción dinámica. Tal interacción es atribuida a los AE posteriores al decaimiento por CE del ${ }^{111} \mathrm{In}\left(\rightarrow{ }^{111} \mathrm{Cd}\right)$. En el primer experimento, las medidas PAC fueron ajustadas con dos interacciones hiperfinas dinámicas utilizando el modelo de Bäverstam y Othaz con un Factor de Perturbación como el de la ecuación (3.2) con la simplificación de que a las dos interacciones observadas se les atribuyeron los mismos parámetros dinámicos $\lambda_{\mathrm{g}} \mathrm{y} \lambda_{\mathrm{r}}$.
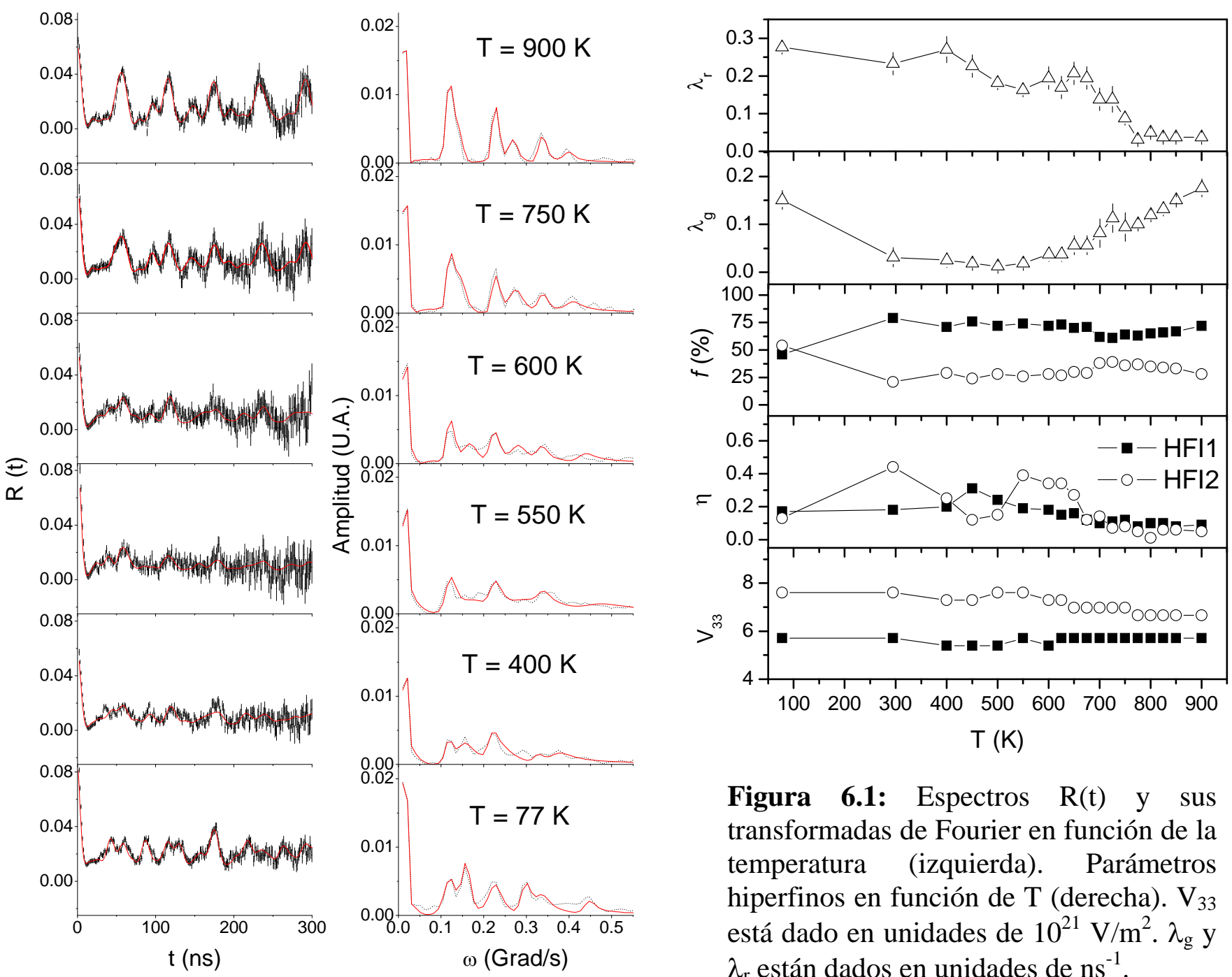

Figura 6.1: Espectros $\mathrm{R}(\mathrm{t}) \quad \mathrm{y}$ sus transformadas de Fourier en función de la temperatura (izquierda). Parámetros hiperfinos en función de $\mathrm{T}$ (derecha). $\mathrm{V}_{33}$ está dado en unidades de $10^{21} \mathrm{~V} / \mathrm{m}^{2}$. $\lambda_{\mathrm{g}} \mathrm{y}$ $\lambda_{\mathrm{r}}$ están dados en unidades de $\mathrm{ns}^{-1}$.

En la Figura 6.1 (derecha) se grafica la dependencia funcional de los parámetros hiperfinos ajustados como función de la temperatura. Puede apreciarse que dos interacciones hiperfinas estables (HFI1 y HFI2) fueron ajustadas en todo el rango de temperaturas, siendo HFI1 la interacción mayoritaria, excepto a $77 \mathrm{~K}$, temperatura en la cual las poblaciones tienden a igualarse. Los parámetros hiperfinos medidos en este experimento para la interacción HFI1 a $900 \mathrm{~K}$ son $f_{1}=72(5) \%, V_{33}=5.71(1)$ y $\eta=0.09(1)$ 
y para HFI2 se obtuvo $f_{2}=28(5) \%, V_{33}=6.66(2)$ y $\eta=0.05(3)$. A temperatura ambiente $(\mathrm{T}=295 \mathrm{~K})$ se obtuvieron las siguientes interacciones: para HFI1 $f_{1}=79(8) \%$, $V_{33}=5.71(5)$ y $\eta=0.18(2)$; mientras que para HFI2 se obtuvo $f_{2}=21(8) \%, V_{33}=7.61(5)$ y $\eta=0.44$ (4) (en todos los casos $\mathrm{V}_{33}$ está dado en unidades de $10^{21} \mathrm{~V} / \mathrm{m}^{2}$ ). En el rango de temperaturas donde las interacciones dinámicas se vuelven apreciables (295 - $700 \mathrm{~K}$ ), los parámetros $\eta$ de ambas interacciones presentan cambios muy importantes llegando a duplicar sus valores ajustados a altas temperaturas. En cuanto a la dependencia de los parámetros dinámicos como función de la temperatura, se puede observar que por encima de $700 \mathrm{~K}$, donde el $\mathrm{AE}$ es despreciable, $\lambda_{\mathrm{r}}$ tiende a cero y $\lambda_{\mathrm{g}}$ aumenta $\left(\tau_{\mathrm{g}}\right.$ decrece), evidenciando una disminución en la intensidad del proceso dinámico y en la vida media de los huecos electrónicos en la vecindad de la sonda ${ }^{111} \mathrm{Cd}$. A $77 \mathrm{~K}$ se observa $\lambda_{\mathrm{r}}$ mantiene su valor ajustado en el rango 295 - $700 \mathrm{~K}$, mientras que $\lambda_{\mathrm{g}}$ crece $\left(\tau_{\mathrm{g}}\right.$ decrece) lo que produce una disminución parcial en la atenuación de la señal. En el siguiente apartado compararemos los dos experimentos realizados en este sistema.

\section{6-1-2 Comparación entre el $1^{\circ}$ y el $2^{\circ}$ experimento}

En el segundo experimento, el rango de bajas temperaturas fue medido de manera más precisa para poder caracterizar ambas interacciones desde los $20 \mathrm{~K}$ hasta los $900 \mathrm{~K}$. En la Figura 6.2 se comparan algunos espectros $\mathrm{R}(\mathrm{t})$ y sus transformadas de Fourier entre ambos experimentos. Como puede apreciarse en ambos casos, a altas temperaturas (700 K-900 K) la interacción dinámica se hace despreciable, en el rango 300 K-400 K la atenuación de la señal se hace máxima y a muy bajas temperaturas (50 K-150 K) la señal se ve recuperada de manera parcial.
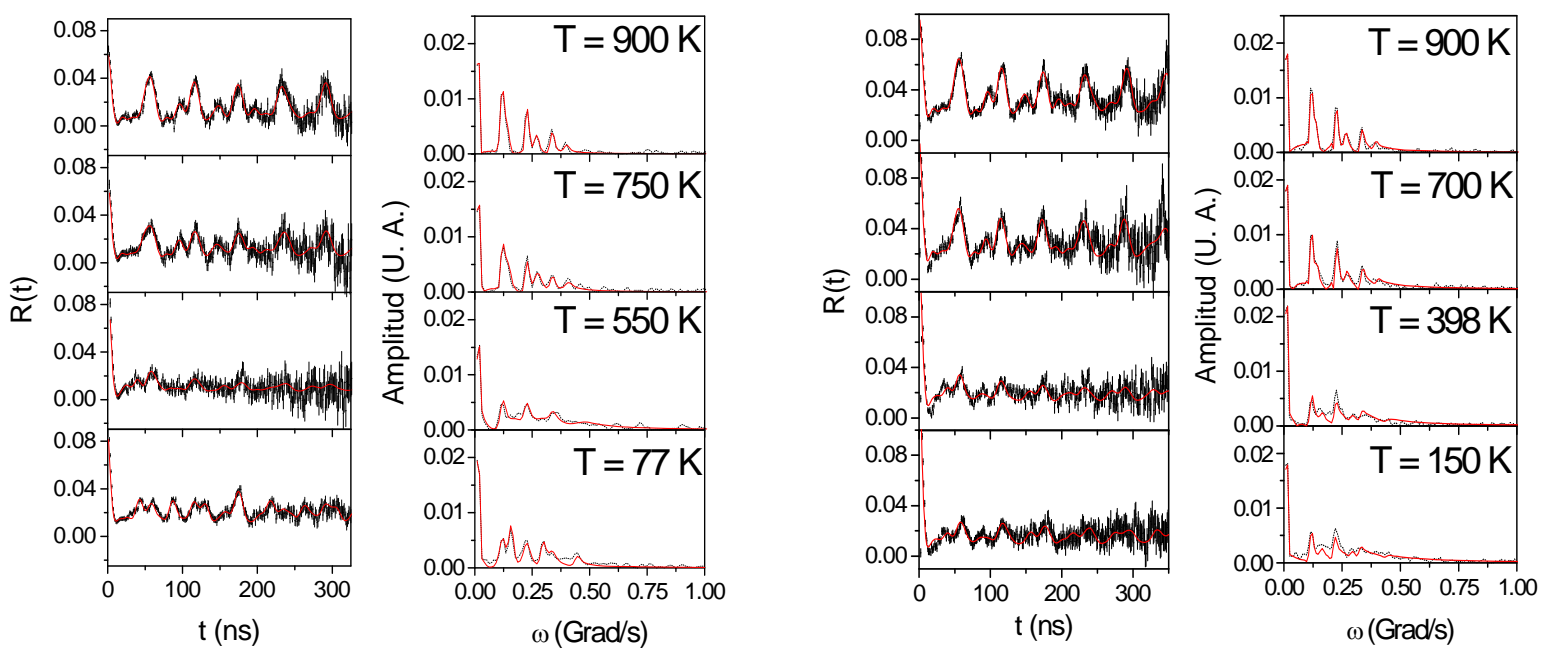

Figura 6.2: Espectros $\mathrm{R}(\mathrm{t})$ y sus transformada de Fourier del $1^{\circ}$ experimento (izquierda) y del $2^{\circ}$ experimento (derecha), en función de la temperatura de medida. 
En la Figura 6.3 se muestran los parámetros hiperfinos en función de la temperatura de medida para ambos experimentos. En ambos casos se caracterizaron dos interacciones dinámicas estables en todo el rango de temperatura, a bajas temperaturas se observa que las poblaciones tienden a igualarse mientras que está tendencia desaparece con el aumento de la temperatura por encima de los 300K. En este caso los espectros de rotación de spín fueron ajustados con un factor dinámico según la ecuación (3.2) y a las dos interacciones se les asignó el mismo par de parámetros dinámicos apra cada temperatura (en los dos experimentos).

En la región de bajas temperaturas del $2^{\circ}$ experimento (región que solo fue medida a $77 \mathrm{~K}$ en el $1^{\circ}$ experimento) se observa que si bien $\lambda_{\mathrm{r}}$ aumenta levemente por debajo de los $300 \mathrm{~K}, \lambda_{\mathrm{g}}$ aumenta conforme la temperatura disminuye hasta $20 \mathrm{~K}$. Este hecho hace que el proceso dinámico se vea disminuido parcialmente debido a que la vida media de los huecos $\left(\tau_{\mathrm{g}}=\lambda_{\mathrm{g}}{ }^{-1}\right)$ en el entorno del Cd decrece a medida que disminuye la temperatura de medida. Cabe destacar que en el $1^{\circ}$ experimento este comportamiento era una supuesta tendencia basada en un solo punto experimental (a $77 \mathrm{~K}$ ) y en el $2^{\circ}$ experimento fue confirmada aumentando los puntos experimentales entre 20 y $300 \mathrm{~K}$. Los parámetros que caracterizan el tensor GCE ( $V_{33}$ y $\eta$ ) se vuelven estables a bajas temperaturas, y en particular, el parámetro de asimetría de la interacción HFI2, el cual varía considerablemente a temperaturas intermedias, se vuelve constante y cercano a cero como a altas temperaturas. Finalmente, las poblaciones de HFI1 y HFI2 tienden a igualarse a bajas temperaturas tal como sucede en la medida de $77 \mathrm{~K}$ del $1^{\circ}$ experimento.
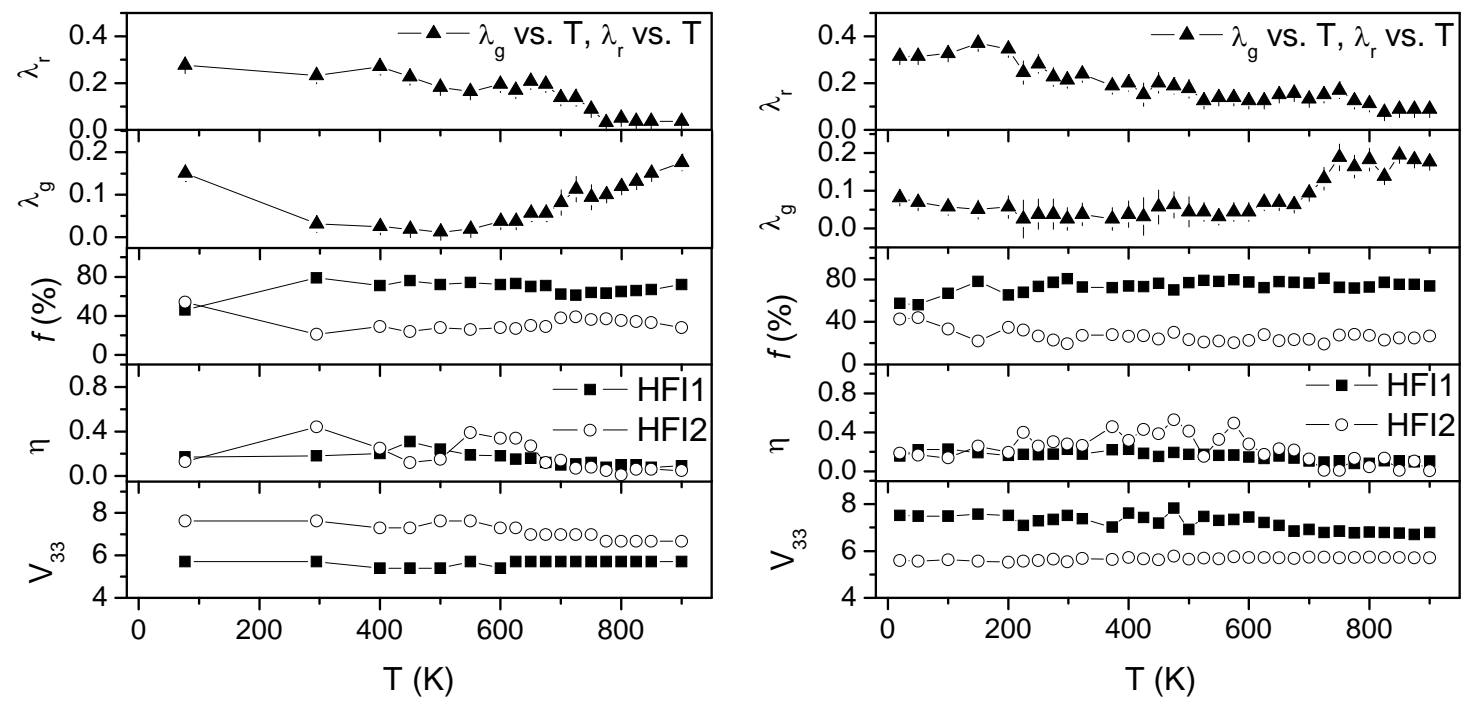

Figura 6.3 : HFIs vs. la temperatura de medida del $1^{\circ}$ experimento (izquierda) y del $2^{\circ}$ experimento (derecha). $V_{33}$ viene dado en unidades de $10^{21} \mathrm{~V} / \mathrm{m}^{2}$. $\lambda_{\mathrm{g}} \mathrm{y} \lambda_{\mathrm{r}}$ están dados en unidades de $\mathrm{ns}^{-1}$. 
Para culminar con la comparación entre ambos experimentos, es útil detallar los parámetros hiperfinos obtenidos a $900 \mathrm{~K}$ en el $2^{\circ}\left(1^{\circ}\right)$ experimento: para HFI1 se obtuvo $f_{1}=73.5(3) \%\left(f_{1}=72(5) \%\right), V_{33}=5.72(1)\left(V_{33}=5.71(1)\right)$ y $\eta=0.10(1)(\eta=0.09(1)) ; \mathrm{y}$ en el caso de HFI2 $f_{2}=26.5(3) \%\left(f_{2}=28(5) \%\right), V_{33}=6.79(1)\left(V_{33}=6.67(2)\right)$ y $\eta=0.01(2)$ $(\eta=0.09(3))$. Finalmente, a temperatura ambiente se caracterizaron las siguientes interacciones en el $2^{\circ}\left(1^{\circ}\right)$ experimento: en el caso de HFI1 se obtuvo $f_{1}=81(6) \%$ $\left(f_{1}=79(8) \%\right), V_{33}=5.54(4)\left(V_{33}=5.72(5)\right)$ y $\eta=0.22(2)(\eta=0.18(2))$; mientras que para HFI2 se tiene $f_{2}=19(6) \%\left(f_{2}=21(8) \%\right), V_{33}=7.52(4)\left(V_{33}=7.63(5)\right)$ y $\eta=0.28(3)$ $\left(\eta=0.44(4)\right.$ ) (en todos los casos $V_{33}$ viene dado en unidades de $10^{21} \mathrm{~V} / \mathrm{m}^{2}$ ). Como se puede apreciar los parámetros hiperfinos obtenidos en ambos experimentos se encuentran en perfecto acuerdo.

Teniendo en cuenta que el proceso dinámico puede llegar a involucrar más de un hueco electrónico y debido a que las dos interacciones se observan en todo el rango de temperatura es de esperar que cada interacción dinámica este caracterizada por parámetros dinámicos diferenciados entre si. Por lo tanto, se procedió a ajustar los espectros del $2^{\circ}$ experimento con parámetros dinámicos independientes para cada interacción. Los espectros $\mathrm{R}(\mathrm{t})$ y sus transformadas se muestran en la Figura 6.4 (superior). Se puede observar que el ajuste es más preciso que el ajuste con un único par de constantes dinámicas, principalmente a temperaturas intermedias donde la atenuación dinámica se hace más importante. En la Figura 6.4 (inferior) izquierda se observan los parámetros hiperfinos vs. T, en este caso se graficaron los parámetros dinámicos $\alpha$ (población de la interacción dinámica) y $\gamma$ (constante de relajación efectiva). En la Fig. 5.4 inferior derecha se muestran los parámetros $\lambda_{\mathrm{g}} \mathrm{y} \lambda_{\mathrm{r}}$. Como puede apreciarse, la interacción HFI2 no presenta comportamiento dinámico a diferencia de lo que sucede con HFI1, es decir que HFI2 es una interacción estática en todo el rango de temperaturas.

De la representación en términos de $\alpha$ y $\gamma$ puede observase que la población dinámica $\alpha$ de HFI1 es muy apreciable (del orden de 0.8) hasta los $600 \mathrm{~K}$. Por encima de ésta, dicho parámetro disminuye conforme aumenta la temperatura, lo cual muestra que el carácter dinámico de HFI1 es muy importante por debajo de $600 \mathrm{~K}$, mientras que a temperaturas superiores dicha interacción se vuelve estática. En cuanto a la constante de relajación efectiva $\gamma$ de HFI1, se observa que es aproximadamente constante entre $200 \mathrm{~K}$ y $700 \mathrm{~K}$ y aumenta a altas y bajas temperaturas. 

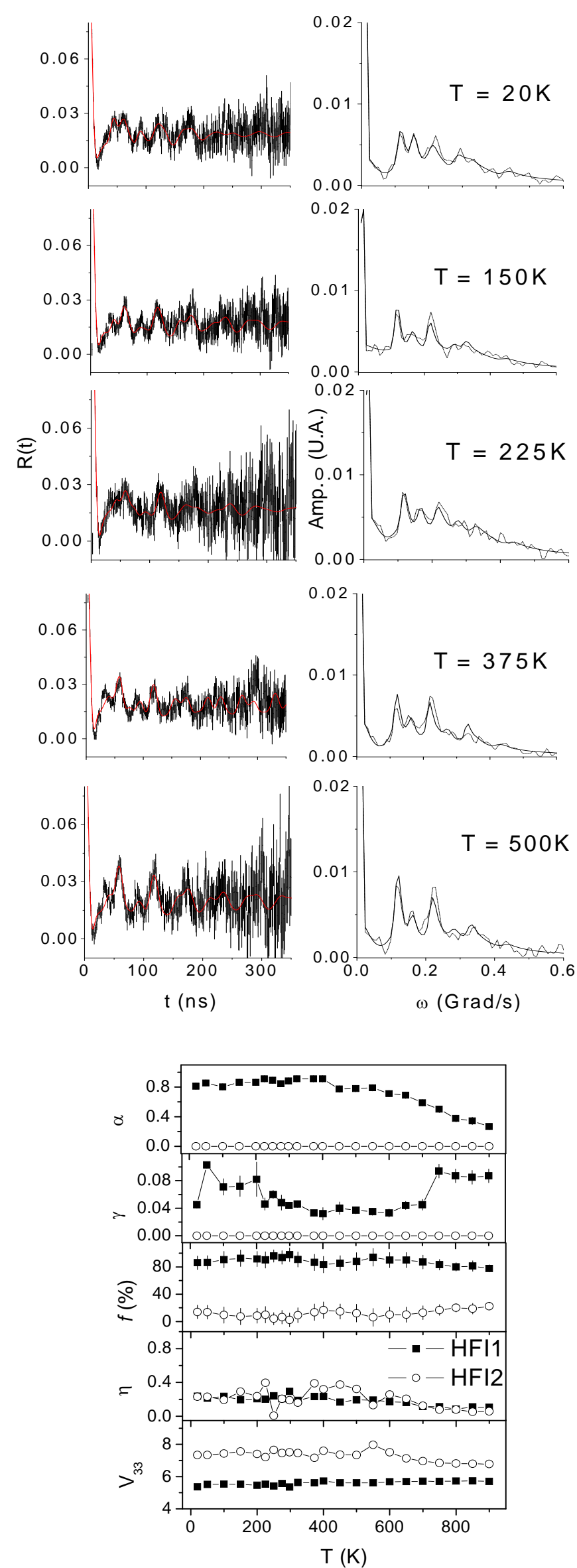
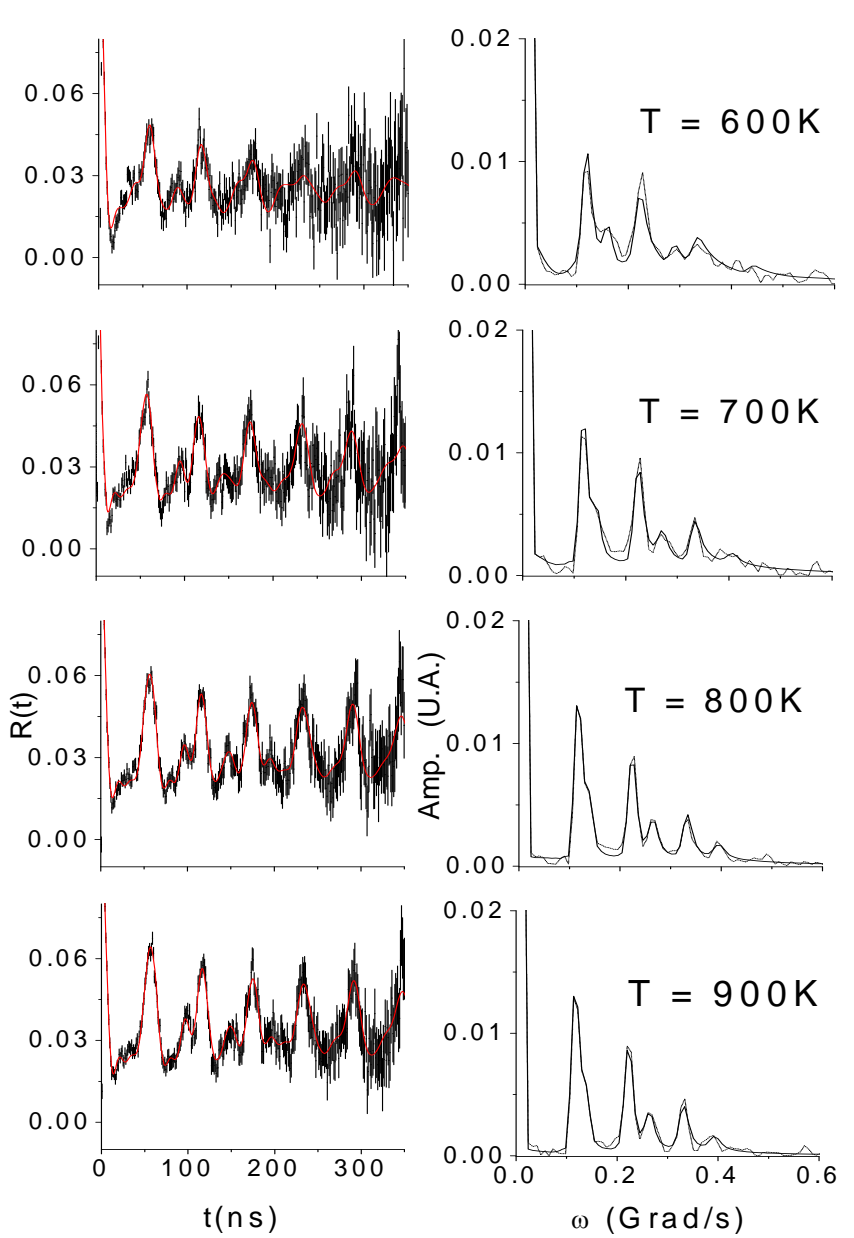

Figura 6.4:. Espectros $\mathrm{R}(\mathrm{t})$ y sus transformadas de Fourier (superior) del $2^{\circ}$ experimento para distintas temperaturas. Parámetros hiperfinos con el factor dinámico parametrizado con $\alpha$ y $\gamma$ vs. T (inferior izquierda). Parámetros hiperfinos con el factor dinámico parametrizado con $\lambda_{\mathrm{g}} \mathrm{y} \lambda_{\mathrm{r}} \mathrm{vs}$. T (inferior derecha). 
Analizando la otra parametrización, vemos que la constante de Abragam y Pound $\lambda_{r}$ de la interacción HFI1 es aproximadamente constante en todo el rango de temperaturas. Mientras que la constante de recuperación $\lambda_{g}$ de HFI1 es pequeña y relativamente constante en presencia de AE y aumenta a altas temperaturas (donde el AE se hace despreciable). Esto se traduce en una vida media del hueco $\tau_{\mathrm{g}}$ lo suficientemente grande como para que la interacción dinámica alcance la ventana temporal PAC por debajo de los $700 \mathrm{~K}$, disminuyendo $\tau_{\mathrm{g}}$ por encima de esta temperatura de modo que no se llega a alcanzar la ventana temporal PAC.

Por último, los parámetros hiperfinos obtenidos con la nueva línea de ajuste con parámetros dinámicos independientes para cada interacción a $900 \mathrm{~K}$ son: para la interacción HFI1 (de población $f_{1}=77.7(5.3) \%$ ) la componente principal es $V_{33}=5.69(1)$ у $\eta=0.11(1)$; mientras que para HFI2 $\left(f_{2}=22.3(5.3) \%\right)$ se tiene $V_{33}=6.78(2)$ y $\eta=0.06(3)$. $\mathrm{Y}$ a temperatura ambiente $(\mathrm{T}=298 \mathrm{~K}$ ) se tiene que para HFI1 la población $f_{1}=97.7(5.0) \%, V_{33}=5.35(4)$ y $\eta=0.29(1)$; y en el caso de HFI2 su población es $f_{2}=2.3(5.0) \%$, su componente principal $V_{33}=7.50(5)$ y $\eta=0.19(4)$ (en todos los casos $V_{33}$ viene dado en unidades de $10^{21} \mathrm{~V} / \mathrm{m}^{2}$ ).

\section{6-2 Resutados PAC en las bixbitas $\mathrm{Sc}_{2} \mathrm{O}_{3}, \mathrm{In}_{2} \mathrm{O}_{3}$ e $\mathrm{Y}_{2} \mathrm{O}_{3}$ medidas con la sonda $\left({ }^{111} \mathrm{In} \rightarrow\right)^{111} \mathrm{Cd}$}

En este apartado se muestran los resultados PAC correspondientes a los óxidos con estructura bixbita. A diferencia del los óxidos $\mathrm{SnO}, \mathrm{SnO}_{2}$ y $\mathrm{ZnO}$ que se discuten en esta Tesis, los cuales tienen un único sitio cristalográfico de catión, las bixbitas presentan dos sitios de catión (C y D) como vimos en el capítulo 4 por lo que a cada interacción hiperfina se le asignarán parámetros dinámicos independientes.

\section{6-2-1 Resultados PAC en $\operatorname{In}_{2} \mathrm{O}_{3}:\left({ }^{111} \mathrm{In} \rightarrow\right)^{111} \mathrm{Cd}$}

En la Figura 6.5 se muestran los espectros PAC y las transformadas de Fourier del sistema $\mathrm{In}_{2} \mathrm{O}_{3}$ dopado con la sonda $\left({ }^{111} \mathrm{In} \rightarrow\right){ }^{111} \mathrm{Cd}$. En dichos espectros se aprecia que por encima de los $700 \mathrm{~K}$ la interacción dinámica se hace despreciable. Así mismo se observa que por debajo de los $100 \mathrm{~K}$ la señal se atenúa pero débilmente, mientras que la interacción dinámica alcanza su máxima intensidad entre 150 K y 400 K. Esto puede contrastarse con la Figura 6.6 donde se muestran la evolución de los parámetros 
hiperfinos en función de la temperatura de medida. En la Figura 6.6 izquierda se grafican las constantes dinámicas $\lambda_{\mathrm{g}}$ y $\lambda_{\mathrm{r}}$ mientras que a la derecha se grafican $\gamma$ y $\alpha$.

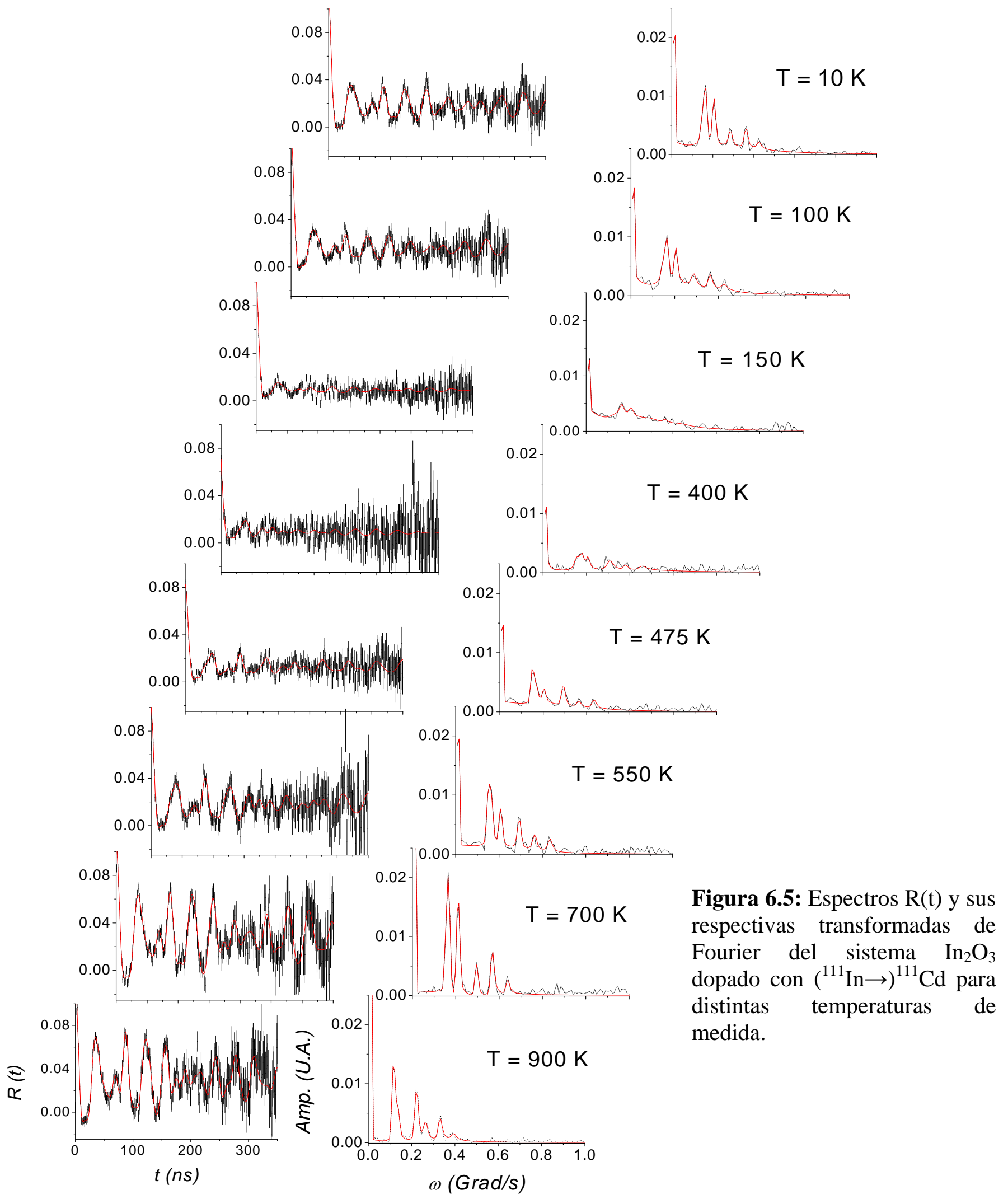


Desde la dependencia funcional de los parámetros hiperfinos con la temperatura se observa que $V_{33}$ y $\eta$ de ambas interacciones se mantienen estables en todo el rango de temperatura. Además, las poblaciones ajustadas en los sitios $C$ y $D$ son aproximadamente cosntantes en todo el rango de temperatura y la relación $f_{C}: f_{D}$ está en muy buen acuerdo con la relación cirstalográfica de la estructura bixbita $\left(f_{C}: f_{D}=3: 1\right)$.

Como se puede observar, en el rango de temperatura donde el AE es apreciable (10 - $600 \mathrm{~K}$ ) el parámetro $\lambda_{\mathrm{r}}$ del sitio C es mayor que el del sitio D. En el caso del sitio D, como $\lambda_{\mathrm{r}} \mathrm{y} \lambda_{\mathrm{g}}$ son pequeños en todo el rango de temperatura, la interacción dinámica en dicho sitio es mucho menor que en el sitio C. En cuanto al parámetro $\lambda_{\mathrm{g}}$ del sitio C, se hace pequeño ( $\tau_{\mathrm{g}}$ grande) entre $100 \mathrm{~K}$ y $600 \mathrm{~K}$, por debajo de $100 \mathrm{~K} \lambda_{\mathrm{g}}$ crece evidenciando una disminución de la interacción dinámica, mientras que por encima de los $600 \mathrm{~K}$ la interacción en el sitio C deviene estática. Entre 100 y $600 \mathrm{~K}$ en el sitio C, $\tau_{\mathrm{g}}$ es suficientemente apreciable como para alcanzar la ventana PAC. Por debajo de este rango, el electrón llega a ocupar el hueco del nivel de impureza antes de que comience dicha ventana temporal. Mientras que por encima de $600 \mathrm{~K}, \lambda_{\mathrm{r}}$ se hace nulo por lo que la interacción se hace estática.

Si ahora observamos la parametrización en términos de $\alpha$ y $\gamma$, vemos que la población de la interacción dinámica $\alpha$ para el sitio D se anula antes que para el sitio C. Y en cuanto a $\gamma$, la constante de relajación es mayor en el sitio C que en el D en todo el rango de temperaturas donde el AE es más apreciable. Además, para el caso del sitio D, $\gamma$ es despreciable lo que implica una interacción dinámica despreciable frente a la intensidad de la interacción dinámica del sitio C. Estos comentarios están en acuerdo con la mayor dependencia del GCE con el estado de carga de la impureza en el sitio asimétrico C respecto del sitio axialmente simétrico D, como veremos en el Capítulo 7.

Por último, los parámetros hiperfinos que caracterizan las interacciones en los sitios C y D (HFIC y HFID, respectivamente) son: en el caso de HFIC a 873 K la población es $f_{C}=77.4(2.0), V_{33}=6.14(2)$ y el parámetro de asimetría $\eta=0.70(1)$; mientras que para HFID se tiene $f_{D}=22.6(2.0), V_{33}=7.58(1)$ y $\eta=0.00$. Y a temperatura ambiente $(\mathrm{T}=298$ K) HFIC está caracterizada por $f_{C}=71.5(4.0), V_{33}=5.83(44)$ y $\eta=0.94(4)$; mientras que HFID presenta una población de $f_{D}=28.5(4.0), V_{33}=7.57(11)$ y $\eta=0.00$ (en todos los 
casos $V_{33}$ viene dado en unidades de $10^{21} \mathrm{~V} / \mathrm{m}^{2}$ ). Estos resultados (a 900 y a $298 \mathrm{~K}$ ) están en perfecto acuerdo con los reportados por Habenitch et al. [Habenicht 1996].
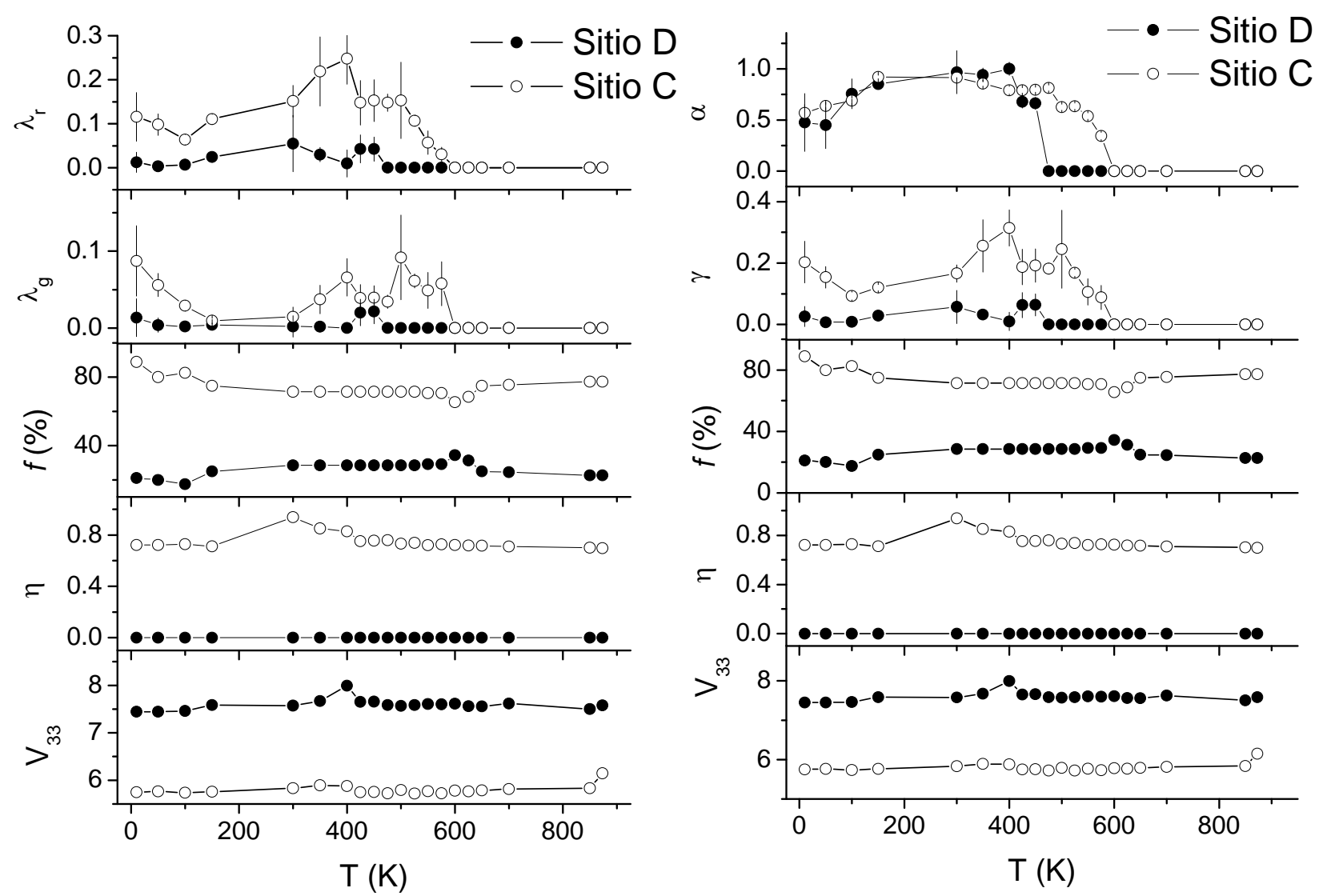

Figura 6.6: Parámetros hiperfinos con el factor dinámico parametrizado con $\alpha$ y $\gamma$ vs. la temperatura de medida T (derecha). Parámetros dinámicos $\lambda_{g}$ y $\lambda_{r}$ vs. T (izquierda). $\mathrm{V}_{33}$ viene dado en unidades de $10^{21} \mathrm{~V} / \mathrm{m}^{2} . \lambda_{g}, \lambda_{r} \mathrm{y}$ $\gamma$ están en unidades de dados en $\mathrm{ns}^{-1}$.

\section{6-2-2 Resultados PAC en $\mathrm{Sc}_{2} \mathrm{O}_{3}:\left({ }^{111} \mathrm{In} \rightarrow\right)^{111} \mathrm{Cd}$}

En este apartado discutiremos los resultados PAC en $\mathrm{Sc}_{2} \mathrm{O}_{3}:{ }^{111} \mathrm{Cd}$. En la Figura 6.7 se muestran los espectros $\mathrm{R}(\mathrm{t})$ y sus respectivas transformadas de Fourier. Como puede apreciarse, el rango de temperaturas donde el AE es apreciable (10 K - 550K) es mayor que en el caso del $\mathrm{In}_{2} \mathrm{O}_{3}$. Además la señal prácticamente no se recupera a temperaturas bajas. En cambio en el caso del $\mathrm{In}_{2} \mathrm{O}_{3}$ la señal se recupera por debajo de los $100 \mathrm{~K}$ e inclusive está muy bien definida. Por encima de los 700K, la interacción dinámica es despreciable aunque a partir de los $650 \mathrm{~K}$ empieza a aparecer la atenuación. 


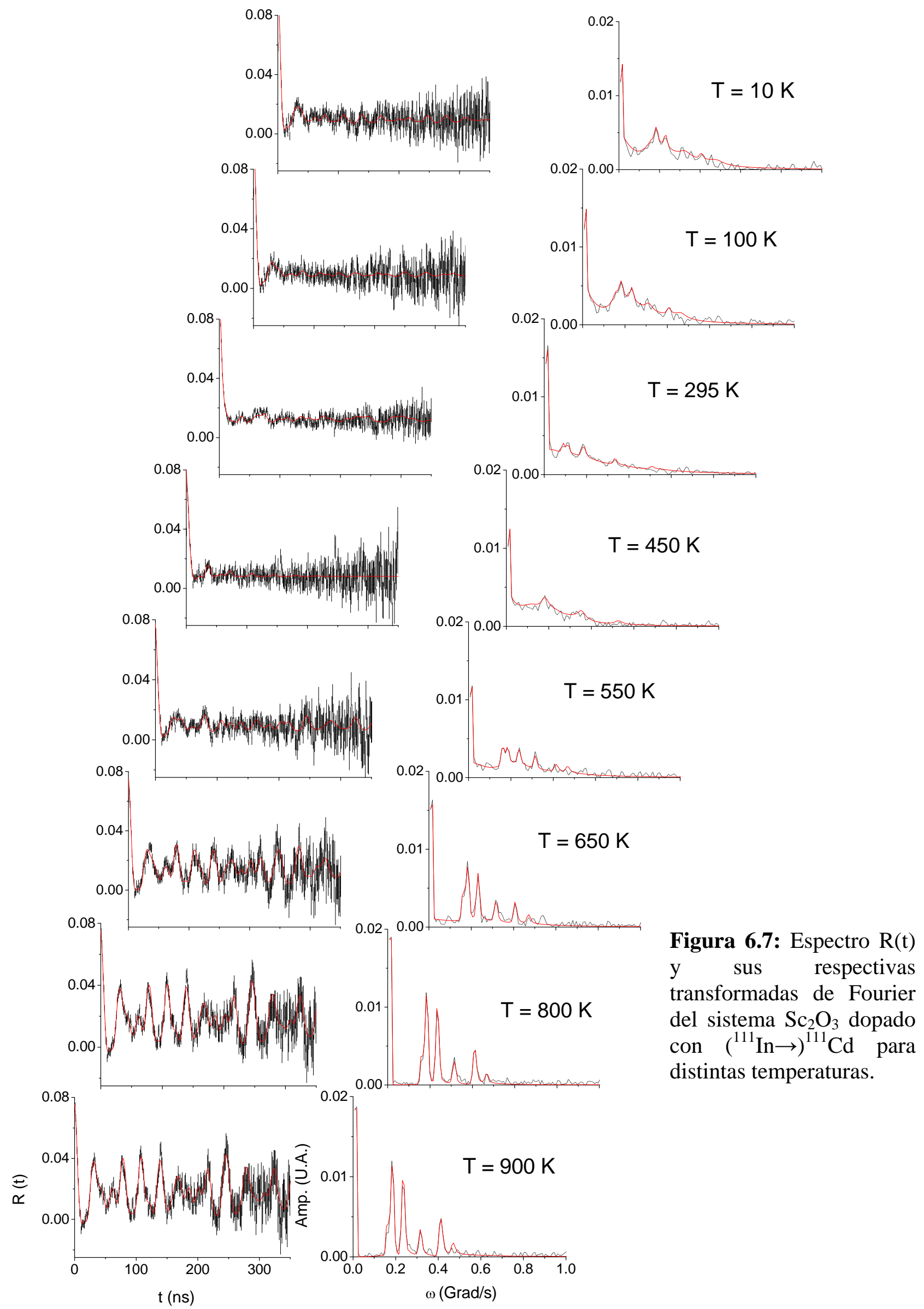


En la Figura 6.8 (izquierda) se grafica la dependencia de los parámetros hiperfinos en función de la temperatura con el Factor de Perturbación parametrizado con $\lambda_{\mathrm{g}} \mathrm{y} \lambda_{\mathrm{r}}$. En la Fig. 5.8 (derecha) se muestra la parametrización dinámica en términos de $\alpha$ y $\gamma$ vs. T. De la dependencia de $\mathrm{V}_{33}$ como función de la temperatura para el caso de la interacción asignada a Cd en sitio D se observa que se mantiene aproximadamente constante en todo el rango de temperatura, mientras que el parámetro $\eta$ del sitio $\mathrm{D}$ se mantuvo fijo con valor nulo debido a la simetría axial del sitio. En el caso de la interacción asignada a $\mathrm{Cd}$ en sitio $\mathrm{C}$, se aprecia que ambos parámetros se mantienen constantes por encima de $500 \mathrm{~K}$. Entre 300 y $500 \mathrm{~K}$, el parámetro $\eta$ del sitio C disminuye abruptamente hasta anularse, mientras que $\mathrm{V}_{33}$ disminuye débilmente. En el rango 200 - $300 \mathrm{~K}, \eta$ aumenta hasta el valor ajustado a altas temperaturas, mientras que $\mathrm{V}_{33}$ disminuye nuevamente hasta $3 \times 10^{21} \mathrm{~V} / \mathrm{m}^{2}$. Finalmente, por debajo de $200 \mathrm{~K}$, ambos parámetros hiperfinos que caracterizan al sitio C alcanzan los valores ajustados a altas temperaturas. En cuanto a las poblaciones ajustadas en los sitios C y D, se observa que se mantienen aproximadamente constantes en todo el rango de temperaturas y en muy buen acuerdo con la relación cristalográfica $\left(f_{C}: f_{D}=3: 1\right)$.

En cuanto a la dependencia de los parámetros dinámicos como función de la temperatura, se puede observar que en el caso del $\mathrm{Sc}_{2} \mathrm{O}_{3}$ el parámetro $\lambda_{\mathrm{r}}$ es apreciable en un mayor rango de temperaturas comparado con el sistema $\operatorname{In}_{2} \mathrm{O}_{3}:\left({ }^{111} \mathrm{In} \rightarrow\right){ }^{111} \mathrm{Cd}$, principalmente para la interacción del sitio C cuya interacción dinámica contribuye predominantemente en la forma de los espectros. Además, $\lambda_{\mathrm{r}}$ del sitio C está por encima del sitio D en el rango de temperaturas donde la relajación dinámica es apreciable, tal como se observó en el óxido $\mathrm{In}_{2} \mathrm{O}_{3}$. El parámetro $\lambda_{\mathrm{g}}$ es similar en ambos sitios y cercano a cero en el rango de temperaturas donde el $\mathrm{AE}$ es importante, mientras que por encima de $700 \mathrm{~K}, \lambda_{\mathrm{g}}$ del sitio C crece (la vida media $\tau_{\mathrm{g}}$ decrece), y en el sitio D $\lambda_{\mathrm{g}} \mathrm{y} \lambda_{\mathrm{r}}$ se anulan debido a que no hay interacción dinámica en $\mathrm{D}$ por encima de dicha temperatura. En el caso del sitio $\mathrm{C}$, por encima de $700 \mathrm{~K}$ el hecho que $\lambda_{\mathrm{r}}$ disminuya y $\lambda_{\mathrm{g}}$ aumente muestran una importante disminución del proceso dinámico en dicho sitio hasta hacerse despreciable [Muñoz 2011].

Si observamos la otra parametrización, vemos que la población $\alpha$ es aproximadamente igual en ambos sitios excepto que en el caso del sitio D empieza a disminuir a menores temperaturas, mientras que la constante de relajación efectiva $\gamma$ en 
todo el rango de temperatura resulta mayor en el sitio $\mathrm{C}$ que en el $\mathrm{D}$ de modo que el proceso dinámico presenta es más apreciable en el sitio C respecto del D. De este modo, la mayor intensidad de la interacción dinámica observada en el sitio C (altamente asimétrico) frente al D (axialmente simétrico) está en acuerdo con la dependencia del GCE con el estado de carga [Muñoz 2009] tal como se verá en el Capítulo 7.

Los parámetros hiperfinos que caracterizan al tensor GCE en los sitios C y D donde se localiza la sonda-impureza $\left({ }^{111} \mathrm{In} \rightarrow\right)^{111} \mathrm{Cd}$ (HFIC y HFID, respectivamente) a $900 \mathrm{~K}$ son: en el caso de HFIC su población es $f_{C}=75 \%$, su componente principal es $V_{33}=6.57(2)$ y el parámetro de asimetría $\eta=0.70(1)$; en el caso de HFID se tiene $f_{D}=25$ $\%, V_{33}=8.23(1)$ y $\eta=0.00$. A $650 \mathrm{~K}$ se obtuvieron los siguientes parámetros: en el caso de HFIC $f=80.6(3) \%, V_{33}=6.47(2)$ y $\eta=0.71(1)$; mientras que para HFID $f=19.4(3) \%$, $V_{33}=8.24(1)$ y $\eta=0.00$. Finalmente, a temperatura ambiente ( $\mathrm{T}=295 \mathrm{~K}$ ) se tiene para HFIC una población $f_{C}=75(4) \%, V_{33}=3.02(6)$ y $\eta=0.72(2)$; y en el caso de HFID los parámetros son $f_{D}=25(4) \%, V_{33}=9.31(3)$ y $\eta=0.00$ (en todos los casos $V_{33}$ viene dado en unidades de $10^{21} \mathrm{~V} / \mathrm{m}^{2}$ ). Cabe destacar que las medidas a $650 \mathrm{~K}$ están en perfecto acuerdo por las reportadas por Bartos et al. a dicha temperatura [Bartos 1991].
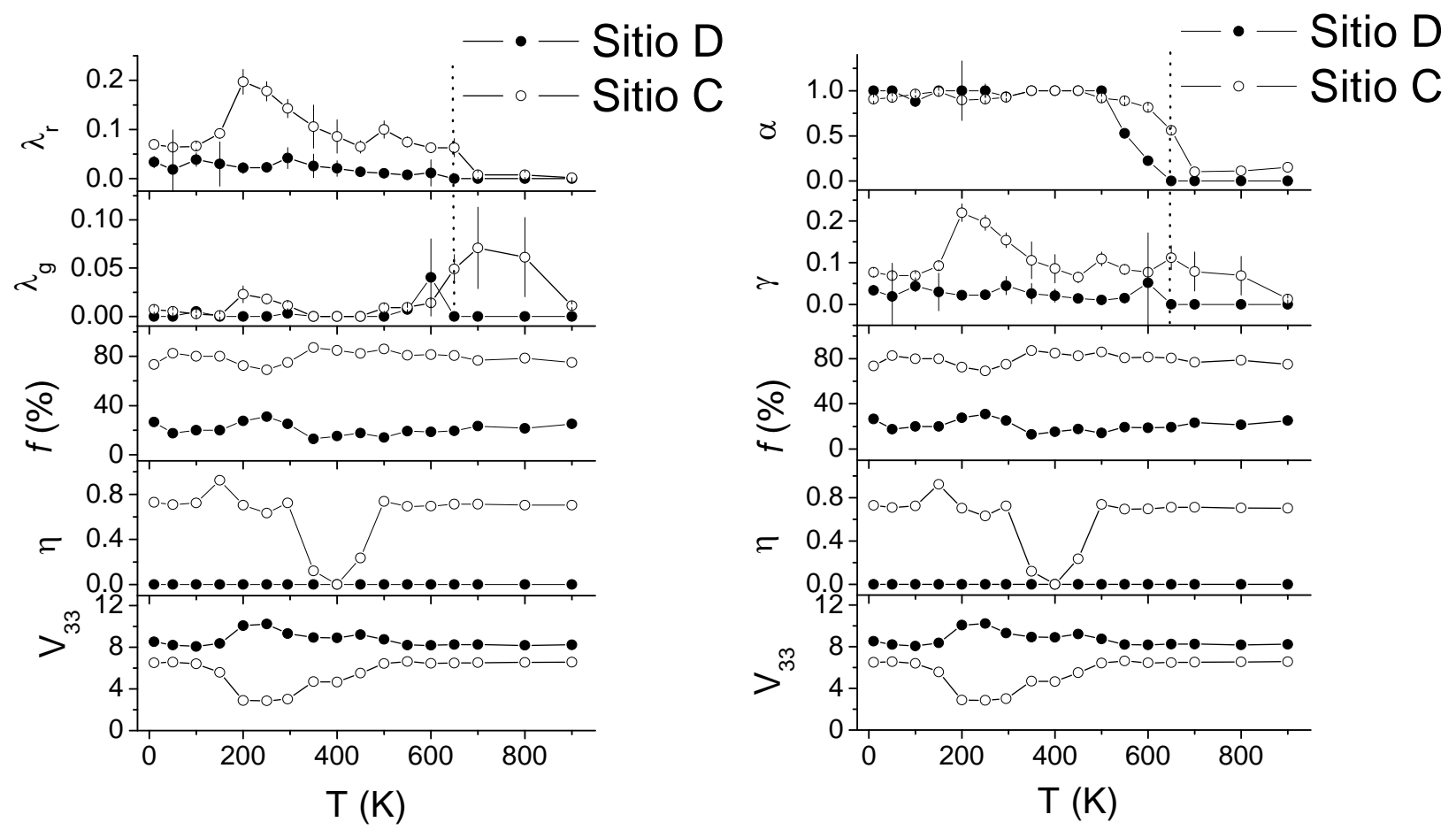

Figura 6.8: Parámetros hiperfinos con el factor dinámico parametrizado con $\alpha$ y $\gamma$ vs. la temperatura $\mathrm{T}$ (derecha). Parámetros dinámicos $\lambda_{\mathrm{g}} \mathrm{y} \lambda_{\mathrm{r}}$ vs. T (izquierda). $\mathrm{V}_{33}$ viene dado en unidades de $10^{21} \mathrm{~V} / \mathrm{m}^{2} . \lambda_{\mathrm{g}}, \lambda_{\mathrm{r}} \mathrm{y} \gamma$ vienen dados en unidades de $\mathrm{ns}^{-1}$. 


\section{6-2-3 Resultados PAC en $\mathrm{Y}_{2} \mathrm{O}_{3}:\left({ }^{111} \mathrm{In} \rightarrow\right)^{111} \mathrm{Cd}$}

A continuación analizaremos los resultados PAC del sistema $\mathrm{Y}_{2} \mathrm{O}_{3}:\left({ }^{111} \mathrm{In} \rightarrow\right)^{111} \mathrm{Cd}$. En la Figura 6.9 se muestran los espectros R(t) y sus respectivas transformadas de Fourier. Como puede apreciarse la atenuación dinámica es muy apreciable en un rango muy amplio de temperaturas como en el caso del $\mathrm{Sc}_{2} \mathrm{O}_{3}(10 \mathrm{~K}-550 \mathrm{~K})$ y recién por encima de los $700 \mathrm{~K}$ se hace despreciable. La señal se recupera muy débilmente por debajo de los $50 \mathrm{~K}$.

En la Figura 6.10 se grafican los parámetros hiperfinos vs. T. En la Fig. 5.10 (izquierda) se grafican los parámetros dinámicos $\lambda_{\mathrm{g}}$ y $\lambda_{\mathrm{r}} \mathrm{y}$, a la derecha, la parametrización en términos de $\alpha$ y $\gamma$. Desde la dependencia de $\mathrm{V}_{33}$ del sitio $\mathrm{D}$ como función de la temperatura se aprecia la componente principal se mantiene constante en todo el rango de temperatura, mientras que $\eta$ se fijó en cero debido a la simetría axial del sitio D. En cuanto a la interacción asignada al sitio $C$, se observa que $V_{33}$ se mantiene aproximadamente constante en todo el rango. Por otro lado, el parámetro $\eta$ del sitio C es estable por encima de $600 \mathrm{~K}$. En el rango 400 - $600 \mathrm{~K}$ disminuye abruptamente hasta anularse y por debajo de los $400 \mathrm{~K}$ varía entorno al valor ajustado a altas temperaturas. Las poblaciones se mantuvieron fijas en sus valores predichos por la estructura.

A partir de la dependencia de los parámetros dinámicos en función de la temperatura, puede apreciarse que el parámetro $\lambda_{\mathrm{r}}$ es mayor en el sitio C que en el D en todo el rango de temperaturas; en particular en el sitio D no hay interacción dinámica por encima de los $700 \mathrm{~K}$. En la región donde el AE es apreciable el parámetro $\lambda_{\mathrm{g}}$ toma valores similares para ambos sitios y, en el caso particular del sitio C por encima de 600 $\mathrm{K}, \lambda_{\mathrm{g}}$ aumenta ( $\tau_{\mathrm{g}}$ disminuye) conforme aumenta la temperatura lo que se traduce en una disminución de la interacción dinámica en el sitio C por encima de los $600 \mathrm{~K}\left(\lambda_{\mathrm{r}}\right.$ se mantiene aproximadamente constante entre 300 y $900 \mathrm{~K}$ ). 

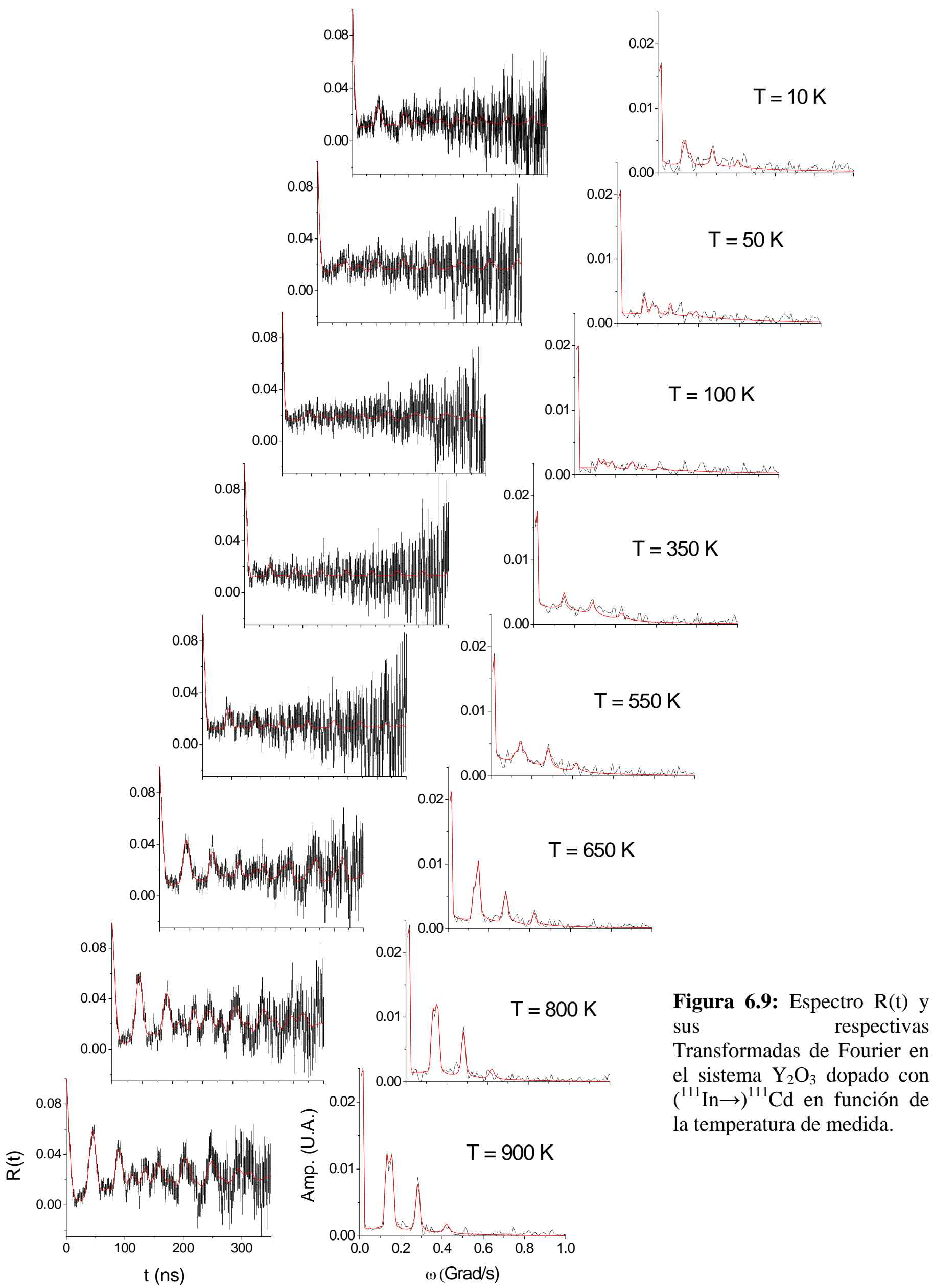
Analicemos ahora la otra parametrización: la población $\alpha$ resulta ser aproximadamente igual para ambos sitios donde el AE es importante mientras que donde éste pierde intensidad, a altas temperaturas, $\alpha$ se hace cero para el sitio D y aproximadamente 0.4 (40 \%) para el C. En cuanto a la constante de relajación efectiva $\gamma$, en todo el rango de temperaturas resulta mayor para el sitio C que para el D. De este modo, la interacción dinámica presenta una mayor intensidad en el sitio C respecto del D. Estas apreciaciones son similares a las observadas en el óxido de Sc y como en las otras dos bixbitas estos resultados están en acuerdo con la dependencia con el estado de carga del GCE observado por el Cd localizado en cada sitio catiónico tal como se verá en el Capítulo 7.
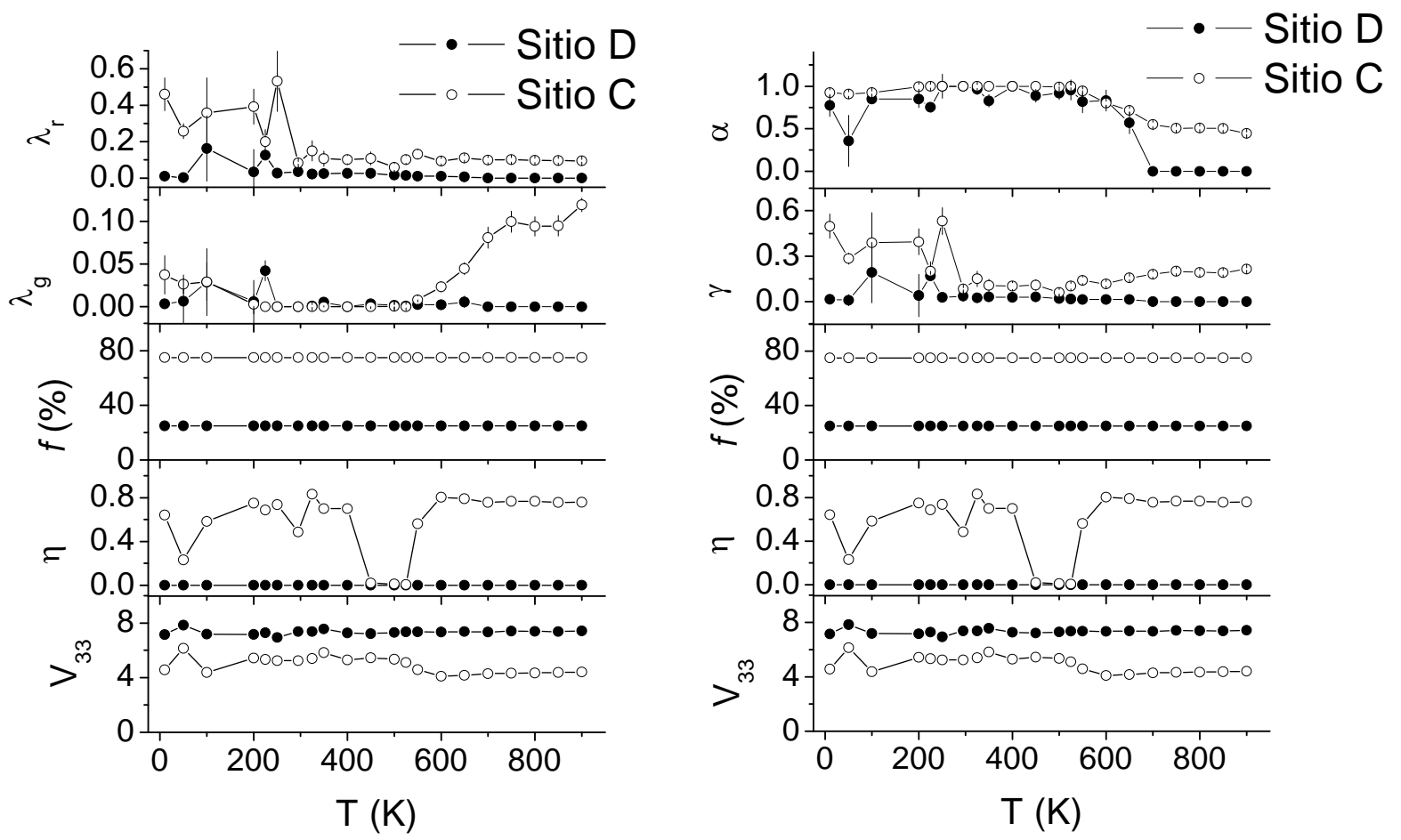

Figura 6.10: Parámetros hiperfinos con el factor dinámico parametrizado con $\alpha$ y $\gamma$ vs. la temperatura $\mathrm{T}$ (derecha). Parámetros dinámicos $\lambda_{\mathrm{g}}$ y $\lambda_{\mathrm{r}}$ vs. T (izquierda). $\mathrm{V}_{33}$ viene dado en unidades de $10^{21} \mathrm{~V} / \mathrm{m}^{2} . \lambda_{\mathrm{g}}, \lambda_{\mathrm{r}} \mathrm{y} \gamma$ vienen dados en unidades de $1 / \mathrm{ns}$.

Por último, los parámetros que caracterizan al GCE en el sitio del ${ }^{111} \mathrm{Cd}$ a $900 \mathrm{~K}$ son: para el sitio C (HFIC) la población es $f_{C}=75 \%, V_{33}=4.41(3)$ y $\eta=0.76(1)$; mientras que para el sitio D (HFID), $f_{D}=25 \%, V_{33}=7.42(1)$ y $\eta=0.00$. Finalmente, a temperatura ambiente $(\mathrm{T}=295 \mathrm{~K})$ la interacción HFIC viene dada por $f_{C}=75 \%, V_{33}=5.35(24) \mathrm{y}$ $\eta=0.49(8)$; en el caso de HFID se tiene $f_{D}=25 \%, V_{33}=7.33(28)$ y $\eta=0.00$. Estas medidas 
están en perfecto acuerdo con las reportadas por Bartos et al. en este sistema [Bartos 1991].

\section{6-3 Resultados PAC del óxido $\mathrm{SnO}_{2}$ medido con la sonda $\left({ }^{111} \mathrm{In} \rightarrow\right){ }^{111} \mathrm{Cd}$}

En la Figura 6.11 se muestran los espectros R(t) y sus transformadas de Fourier correspondientes al sistema $\mathrm{SnO}_{2}:\left({ }^{111} \mathrm{In} \rightarrow\right){ }^{111} \mathrm{Cd}$. En todo el rango de temperaturas coexisten dos interacciones hiperfinas dinámicas (HFI1 y HFI2), en las regiones de bajas y altas temperaturas HFI1 resulta mayoritaria, mientras que a temperaturas intermedias tienden a igualarse, excepto en el rango 300 - $500 \mathrm{~K}$ donde HFI2 resulta mayoritaria. Cabe destacar que la estructura rutilo presenta un único sitio cristalográfico de catión axialmente simétrico. Por otro lado, en este sistema el AE se hace despreciable recién por encima de los $900 \mathrm{~K}$. Por debajo de los $650 \mathrm{~K}$ la atenuación dinámica es muy importante y crece a medida que disminuye la temperatura de medida hasta los $10 \mathrm{~K}$.

En la Figura 6.12 (izquierda) se grafican los parámetros hiperfinos vs. T, en particular se muestran los parámetros dinámicos $\lambda_{\mathrm{g}} \mathrm{y} \lambda_{\mathrm{r}} \mathrm{y}$, en la Fig. 5.12 (derecha) se muestra la parametrización en términos de $\alpha$ y $\gamma$. Se ve claramente cómo tiende a desaparecer la población de HFI2 por encima de los 900 K y por debajo de $300 \mathrm{~K}$. Por debajo de 900 y hasta los 500 K, la población de HFI2 compite con la de HFI1 entorno al 50\%. Finalmente entre 300 y $500 \mathrm{~K}$ HFI2 se hace mayoritaria. En cuanto a los parámetros hiperfinos $V_{33}$ y $\eta$, se aprecia que en el caso de HFI1 se mantienen aproximadamente constantes en todo el rango de temperaturas; mientras que en el caso de HFI2 tanto la componente $V_{33}$ como el parámetro $\eta$ presentan una importante variación por debajo de 900 K, es decir donde HFI2 tiene carácter dinámico.

En la Fig. 5.12 (izquierda) puede observarse que el parámetro $\lambda_{\mathrm{r}}$ de HFI2 es mayor al de HFI1 desde $300 \mathrm{~K}$ hasta $900 \mathrm{~K}$ y, por debajo de $300 \mathrm{~K}$, $\lambda_{\mathrm{r}}$ de ambas interacciones se hace máxima. Además, este parámetro para la interacción HFI1 se anula por encima de los $650 \mathrm{~K}$ mientras que para HFI2 se anula recién por encima de los $900 \mathrm{~K}$. En el caso del parámetro $\lambda_{\mathrm{g}}$ de HFI1, se anula por encima de los $700 \mathrm{~K}$, mientras que entre 400 y $600 \mathrm{~K}$ se maximiza, finalmente por debajo de $300 \mathrm{~K}$ vuelve a hacerse despreciable. En cuanto al parámetro $\lambda_{\mathrm{g}}$ de la interacción HFI2 se hace máximo entre 10 y $300 \mathrm{~K}$ y por encima de esta temperatura se hace despreciable. Por lo tanto, por encima de 900 K ninguna de las interacciones presentan carácter dinámico; entre 600 y $900 \mathrm{~K}$ la atenuación de la señal es parcial debido a que solo HFI1 presenta carácter dinámico; y 
por debajo de $600 \mathrm{~K}$ la atenuación es muy importante y se mantiene aproximadamente constante hasta los $10 \mathrm{~K}$ puesto que en dicho rango ambas interacciones son dinámicas y de intensidad muy apreciable.

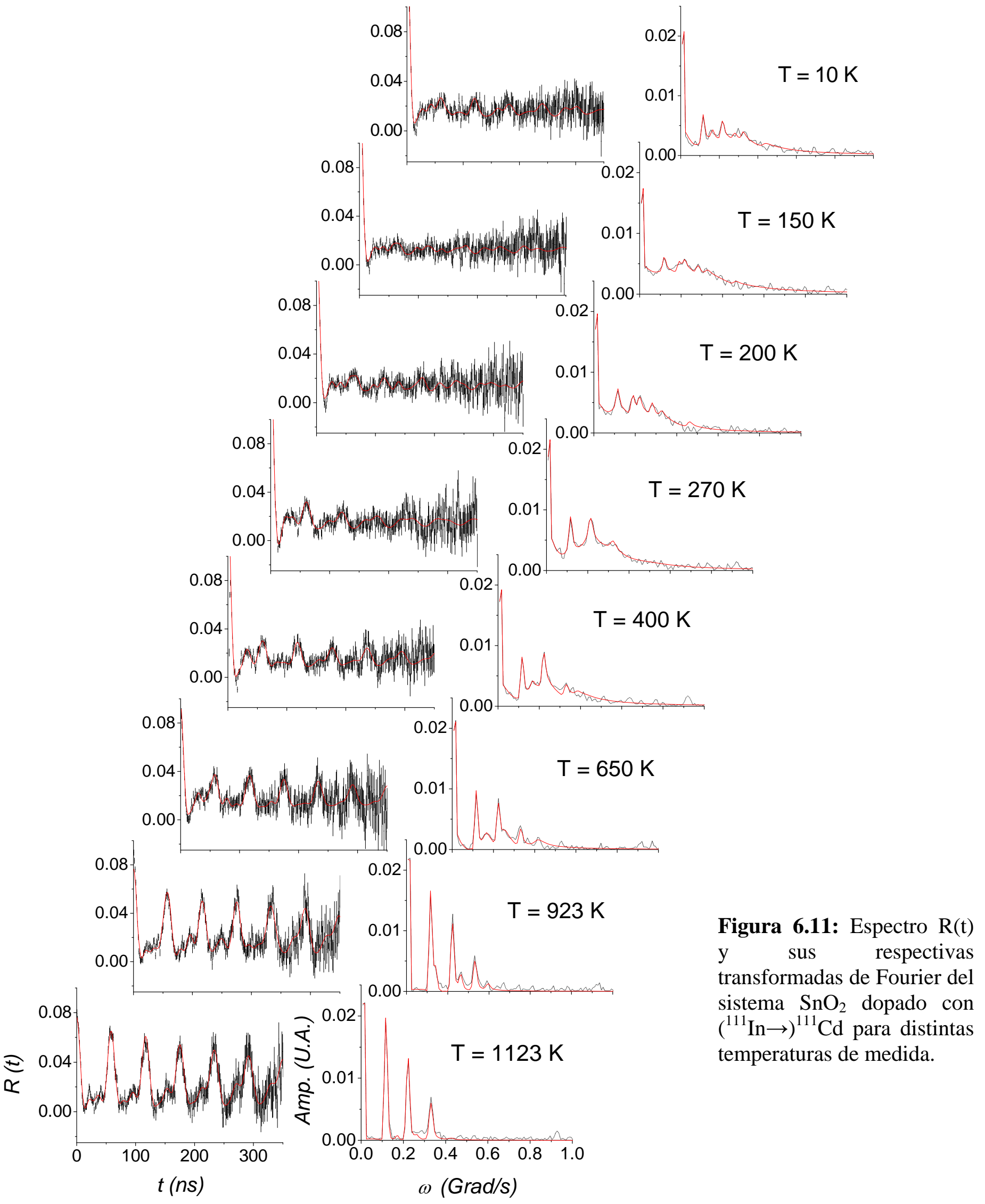



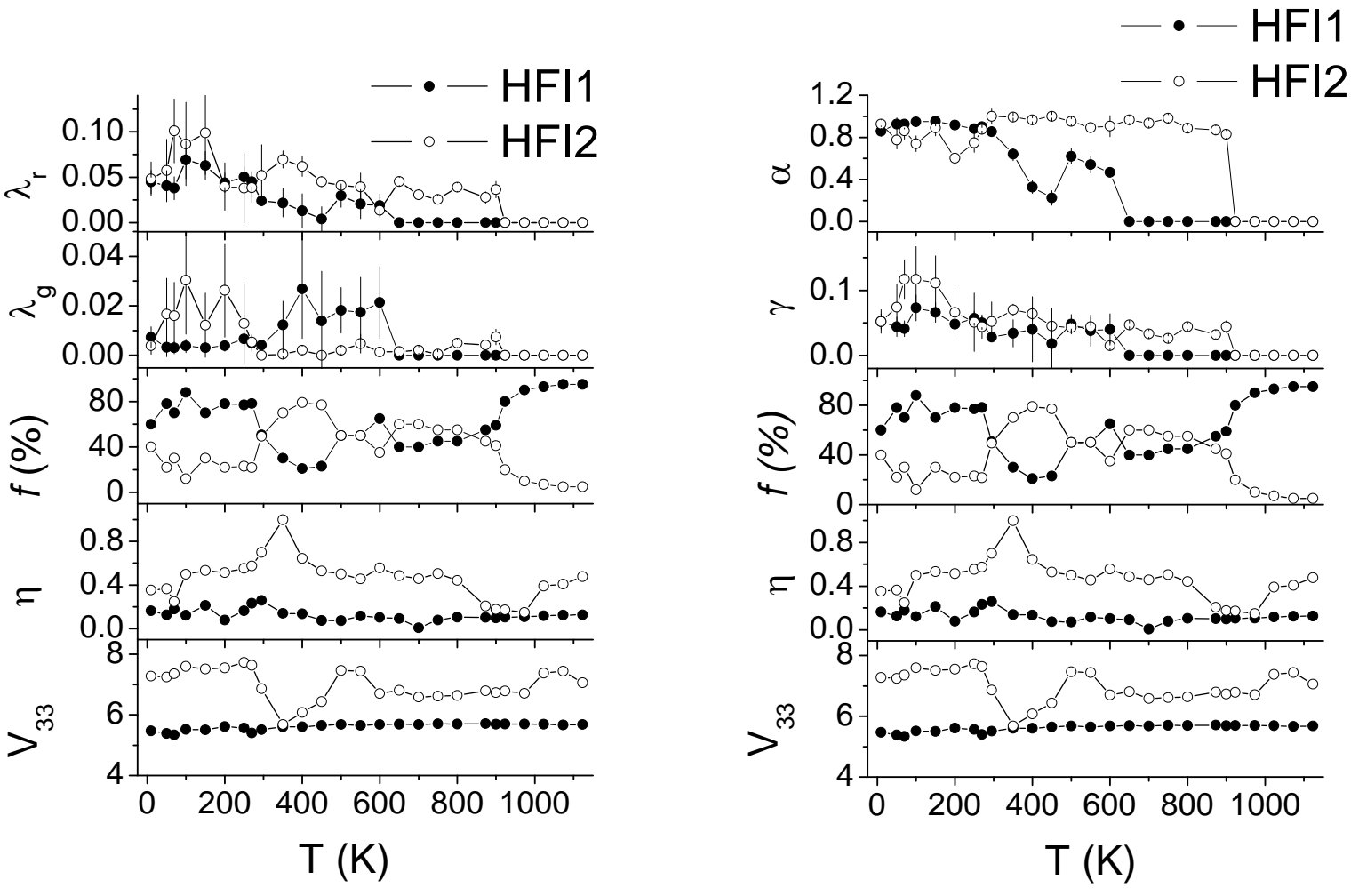

Figura 6.12: Parámetros hiperfinos con el factor dinámico parametrizado con $\alpha$ y $\gamma$ vs. T (derecha). Parámetros dinámicos $\lambda_{\mathrm{g}} \mathrm{y} \lambda_{\mathrm{r}}$ vs. la temperatura $\mathrm{T}$ (izquierda). $\mathrm{V}_{33}$ viene dado en unidades de $10^{21} \mathrm{~V} / \mathrm{m}^{2} . \lambda_{\mathrm{g}}, \lambda_{\mathrm{r}} \mathrm{y} \gamma$ vienen dados en unidades de $\mathrm{ns}^{-1}$.

En un todo de acuerdo con lo descripto, en la Figura 6.12 (derecha) se puede apreciar que por encima de $700 \mathrm{~K}$ la población dinámica $\alpha$ de HFI1 se hace cero y por debajo de $700 \mathrm{~K}$ crece hasta hacerse cercano a la unidad entre 10 y 300K. En el caso de HFI2, $\alpha$ se anula recién por encima de $900 \mathrm{~K}$ y en el rango 10 - $900 \mathrm{~K}$, el parámetro $\alpha$ es siempre cercano a 1. En cuanto a la constante de relajación efectiva $\gamma$ de HFI1 resulta diferente de cero por debajo de los $650 \mathrm{~K}$ y aproximadamente constante. Finalmente, el parámetro $\gamma$ de HFI2 es diferente de cero por debajo de 900 K y alcanza su máximo por debajo de los $650 \mathrm{~K}$ superando a la constante $\gamma$ de HFI1.

Por último, se detallan las interacciones hiperfinas medidas en $\mathrm{SnO}_{2}$ rutilo. La interacción mayoritaria a altas temperaturas (HFI1) está caracterizada a $1123 \mathrm{~K}$ por una fracción $f_{1}=95(1) \%$, una componente principal $V_{33}=5.68(1)$ y un parámetro de asimetría $\eta=0.13(1)$. La interacción minoritaria (HFI2) a 1123 K está caracterizada por $f_{2}=5(1) \%, V_{33}=7.06(1)$ y $\eta=0.48(3)$. A temperatura ambiente ( $\left.\mathrm{T}=295 \mathrm{~K}\right)$, las interacciones 1 y 2 toman los siguientes valores: para HFI1 se obtuvo $f_{1}=38(3) \%$, $V_{33}=5.64(5)$ y $\eta=0.23(2)$; mientras que para HFI2 se tiene $f_{2}=62(3) \%, V_{33}=7.53(5)$ y 
$\eta=0.67(1)$. Teniendo en cuenta que en los rangos de temperatura $10-250 \mathrm{~K}$ y 873 1123 K HFI1 es mayoritaria, resulta interesante caracterizar las interacciones a $250 \mathrm{~K}$ : HFI1 toma los valores $f_{1}=77(3) \%, V_{33}=5.56(3)$ y $\eta=0.23(2)$; mientras que en el caso de HFI2 se tiene $f_{2}=23(3) \%, V_{33}=7.53(3)$ y $\eta=0.67(1)$ (en todos los casos $V_{33}$ está dado en unidades de $10^{21} \mathrm{~V} / \mathrm{m}^{2}$ ). Los parámetros hiperfinos de la interacción HFI1 están en perfecto acuerdo con las medidas reportadas por Wolf et al. [Wolf 1986]. Los autores reportan que dicha interacción se hace máxima por encima de los $1000 \mathrm{~K}$, resultado similar al encontrado por nosotros. Sin embargo a muy bajas temperaturas reportan una población de HFI1 del 27 \%, mientras que en nuestro caso es mayoritaria por debajo de los $300 \mathrm{~K}$.

\section{6-4 Resultados PAC del óxido ZnO medido con la sonda $\left({ }^{111} \mathrm{In} \rightarrow\right){ }^{111} \mathrm{Cd}$}

En este apartado se describen los resultados PAC obtenidos en el óxido $\mathrm{ZnO}$ dopado con Cd. En la Figura 6.13 se detallan los espectros R(t) y sus respectivas transformadas de Fourier en función de la temperatura de medida.

En todo el rango de temperatura (77 K - 1075 K) una sola interacción hiperfina fue suficiente para describir los espectros. En los ajustes, la distribución de la interacción fue fijada en su valor medido a $1075 \mathrm{~K}$. Se aprecia que a $77 \mathrm{~K}$ y a $1075 \mathrm{~K}$ el espectro $\mathrm{R}(\mathrm{t})$ no presenta atenuación en su señal, mientras que a partir de $295 \mathrm{~K}$ comienza a apreciarse el proceso de AE y se mantiene constante hasta los $1000 \mathrm{~K}$ inclusive. Sin embargo se observa que la atenuación en la oscilación es débil.

En la Figura 6.14 se grafican los parámetros hiperfinos ajustados en función de la temperatura. Como se dijo anteriormente, una sola interacción fue ajustada cuyo parámetro de asimetría es cercano a cero en todo el rango de temperaturas y en acuerdo con la simetría puntual del sitio catiónico del ZnO. Desde la dependencia de $\mathrm{V}_{33}$ y $\eta$ como función de la temperatura se observa que ambos parámetros se mantienen estables en todo el rango de temperaturas, en particular $\eta$ es cercano a cero tal como predice la estructura hexagonal del monóxido de Zinc. 


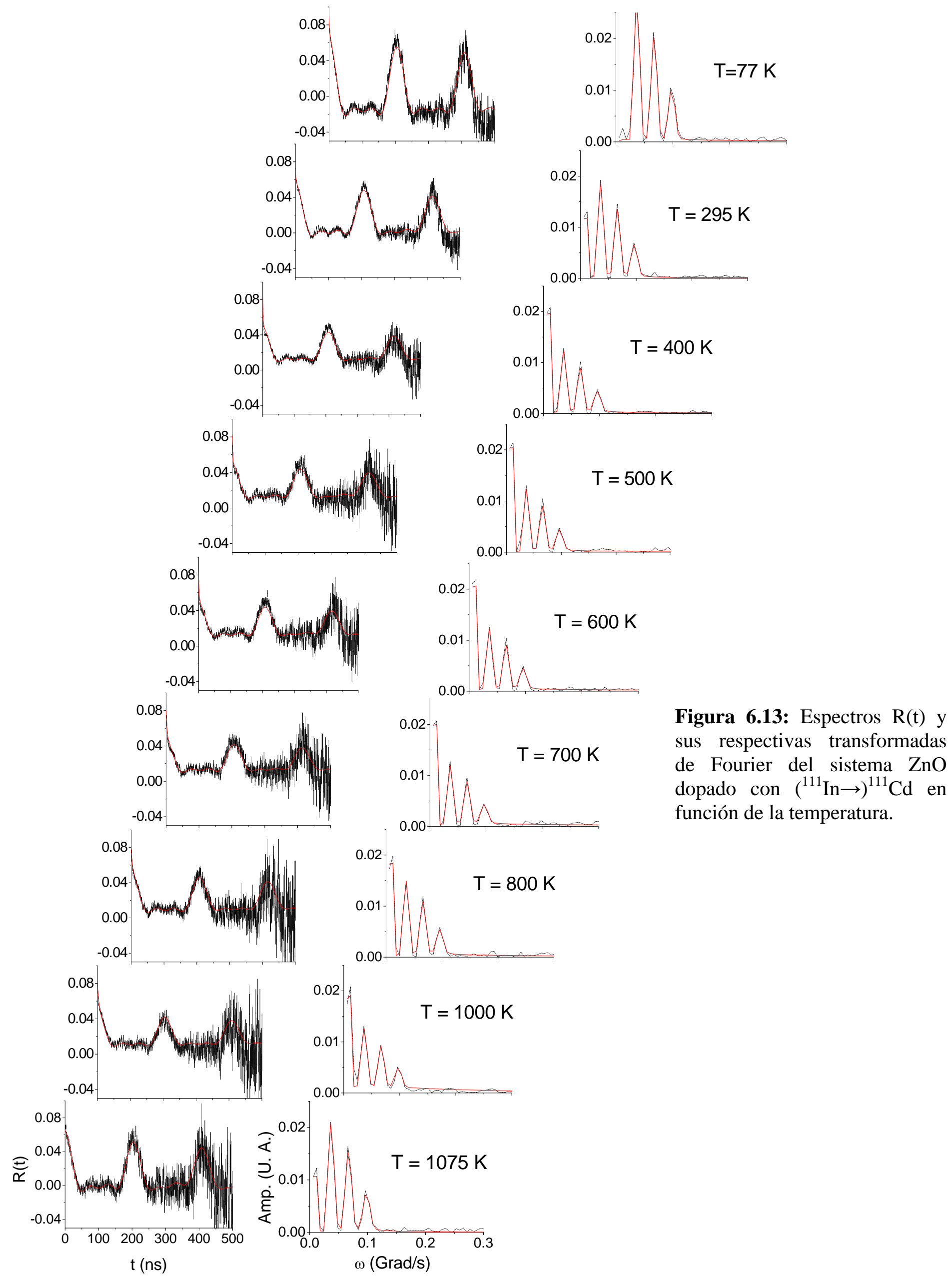


Desde la dependencia de los parámetros dinámicos en función de la temperatura se observa que entre 295 K y $1000 \mathrm{~K}$, donde el AE es débilmente apreciable, el parámetro $\lambda_{r}$ es muy importante aumentando a medida que la temperatura decrece, de hecho alcanza valores máximos similares a los medidos en las bixbitas $\mathrm{Sc}_{2} \mathrm{O}_{3}$ e $\operatorname{In}_{2} \mathrm{O}_{3}$ en las cuales la atenuación es muy importante. Por otro lado, la constante de recuperación $\lambda_{g}$ resulta muy apreciable en el mismo rango de temperaturas y presenta un comportamiento similar al observado en la constante de relajación de Abragam y Pound $\lambda_{\mathrm{r}}$. Cabe destacar que los valores de $\lambda_{g}$ ajustados resultan del orden de 10 veces mayores a los valores observados en los otros óxidos. Teniendo en cuenta que la vida media $\tau_{g}$ de los huecos electrónicos en el entorno de la sonda es el inverso de la constante de recuperación $\left(\tau_{g}=\lambda_{g}{ }^{-1}\right)$, la vida media de los huecos observados en el rango de temperaturas donde el AE es apreciable resulta muy pequeña (10 veces menor a la vida media observada en los otros óxidos) por lo que los huecos electrónicos son ocupados antes del inicio de la ventana temporal PAC, justificando la débil atenuación observada [Muñoz 2010].
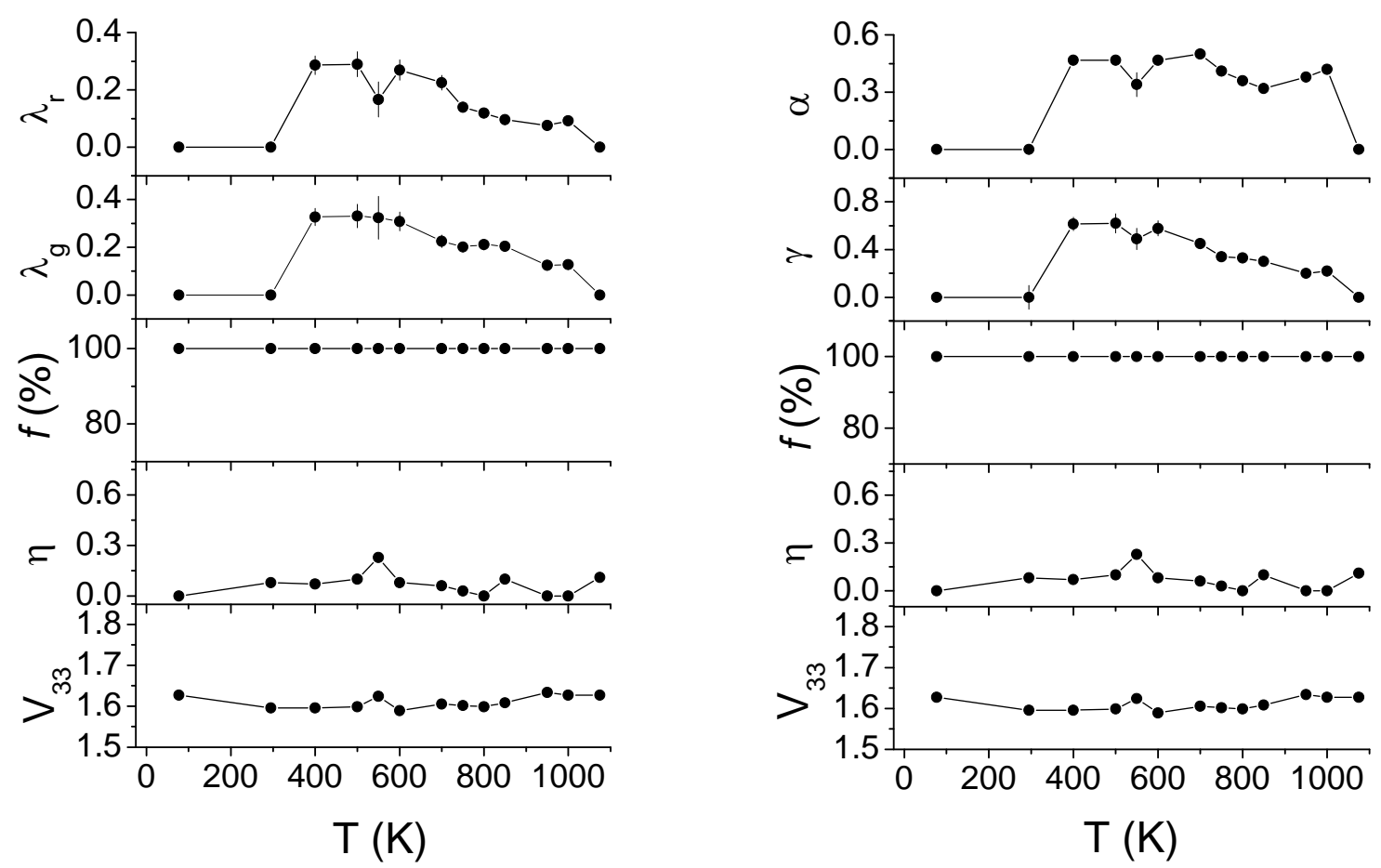

Figura 6.14: Parámetros hiperfinos con el factor dinámico parametrizado con $\alpha$ y $\gamma$ vs. la temperatura T (derecha). Parámetros dinámicos $\lambda_{\mathrm{g}} \mathrm{y} \lambda_{\mathrm{r}}$ vs. T (izquierda). $\mathrm{V}_{33}$ está dado en $10^{21} \mathrm{~V} / \mathrm{m}^{2} . \lambda_{\mathrm{g}}, \lambda_{\mathrm{r}} \mathrm{y} \gamma$ vienen dados en unidades de $\mathrm{ns}^{-1}$. 
Por último, se detallan los parámetros hiperfinos obtenidos a $1075 \mathrm{~K}$ y a temperatura ambiente ( $\mathrm{T}=295 \mathrm{~K}$ ). A $1075 \mathrm{~K}$ se obtuvo una componente principal $V_{33}=1.63(1)$ y un parámetro de asimetría $\eta=0.11(3)$, y a temperatura ambiente se tiene los siguientes parámetros $V_{33}=1.60(1)$ y $\eta=0.08(3)$. Estas medidas están en perfecto acuerdo con las reportadas por Mercurio et al. en ZnO policristalino [Mercurio 2007] y por Rita et al. en ZnO monocristalino [Rita 2004].

\section{Referencias}

[Muñoz 2007] E.L. Muñoz, A.W. Carbonari, L.A. Errico, A.G. Bibiloni, H. M. Petrilli, M. Rentería, Hyp. Int. 178, 37 (2007).

[Habenicht 1996] S. Habenicht, D. Lulascu, M. Uhrmacher, L. Ziegeler. K.P. Lieb y ISOLDE collaboration, Z. Phys. B 101, 187 (1996).

[Muñoz 2011] E.L. Muñoz, D. Richard, P.D. Eversheim and M. Rentería, Hyperfine Interact. 197, 181 (2011).

[Muñoz 2009] E.L. Muñoz, D. Richard, L.A. Errico, M. Rentería, Physica B 404, 2757 (2009).

[Bartos 1991] A. Bartos, K.P. Lieb, A.F. Pasquevich, M. Uhrmacher e ISOLDE collaboration, Phys. Lett. A 157, 513 (1991).

[Wolf 1986] Wolf, H., Deubler, S., Forkel, D., Foettinger, H., Iwatschenko-Borho, M., Meyer, M., Renn, M., Witthuhn, W., Mat. Sci. Forum. 10-12, 863-868 (1986).

[Muñoz 2010] E.L. Muñoz, M.E. Mercurio, M.R. Cordeiro, L.F.D. Pereira, A.W.

Carbonari, and M. Rentería, Physica B (2010) (enviado para su publicación).

[Mercurio 2007] M.E. Mercurio, A.W. Carbonari, M.R. Cordeiro, and R.N. Saxena, Hyperfine Interact 178, 247 (2007).

[Rita 2005] E. Rita, J. G. Correia, U. Wahl, E. Alves, A. M. L. Lopes, J. C. Soares and The ISOLDE Collaboration, Hyperfine Interact. 158, 395 (2005). 


\section{Capítulo $\mathbf{N}^{0} 7$}

\section{Cálculos FP-APW+lo}

\section{7-1 Cálculos ab initio en los sistemas puros}

Los cálculos FP-APW+lo tanto en los sistemas puros como en los dopados fueron realizados con el código WIEN2k en las aproximaciones LDA, GGA y CW-GGA.

En principio se estudió la convergencia de los parámetros hiperfinos con el parámetro de cálculo RKMAX, el cual gobierna el tamaño de la base. Cabe destacar que para los cálculos de los sistemas puros y dopados se utilizaron 50 puntos $k$ en la zona irreducible de Brillouin (IBZ) de la red recíproca, debido a que se corroboró que con dicho valor está asegurada la convergencia en puntos $k$. Posteriormente, los parámetros de red fueron refinados minimizando la energía total de la celda y fueron comparados con los parámetros experimentales reportados en el Capítulo 3. Los parámetros internos de cada estructura cristalina también fueron optimizados vía la minimización de las fuerzas cuánticas calculadas sobre cada ión, los cuales fueron desplazados mediante un proceso de amortiguamiento de Newton según estas fuerzas, este procedimiento se realizó de manera iterativa hasta que las fuerzas se hicieron menores a un valor de corte de $0.025 \mathrm{eV} / \AA$.

\section{7-1-1 Cálculos en los óxidos $\operatorname{In}_{2} \mathrm{O}_{3}, \mathrm{Y}_{2} \mathrm{O}_{3}$ y $\mathrm{Sc}_{2} \mathrm{O}_{3}$ puros}

$\mathrm{In}_{2} \mathrm{O}_{3}$ puro: Inicialmente se estudió la convergencia de la componente principal del GCE $V_{33}$, del parámetro de asimetría $\eta$ y de la energía total de la supercelda en función del parámetro RKMAX, esto se muestra en la Figura 7.1 (a). Posteriormente se calculó la energía total de la supercelda en función del parámetro de red (con RKMAX=7) para encontrar el parámetro a que minimice la energía para contrastarlo con su valor experimental (ver Figura 7.1 (b)). Finalmente, en la Tabla 7.1 se muestran los parámetros internos optimizados. 
Como puede apreciarse en la Figura 7.1 (a), los parámetros hiperfinos que caraterizan el tensor GCE y la energía del sistema se pueden considerar convergidos para $\mathrm{RKMAX}=7$

Como se puede apreciar en la Tabla 7.1, los parámetros estructurales $u, x, y, z$ obtenidos con el método FP-APW+lo están en muy buen acuerdo con los valores experimentales. Este cálculo es válido a $0 \mathrm{~K}$ por lo cual se supone a los iones fijos en sus posiciones de red. Es por ésto que el parámetro $a$ teórico esperado debería ser menor al valor experimental reportado en la literatura [Marezio 1966] medido a temperatura ambiente. Sin embargo el valor de a calculado en la aproximación CW-GGA resulta mayor al experimental aunque la diferencia resulta menor a $0.05 \AA$. Teniendo en cuenta el perfecto acuerdo citado anteriormente, para calcular el sistema $\operatorname{In}_{2} \mathrm{O}_{3}$ dopado con Cd vamos a utilizar los parámetros de red experimentales. Además, los parámetros hiperfinos teóricos serán comparados con los experimentales los cuales son medidos en función de la temperatura, y en particular, a temperatura ambiente.

(a)

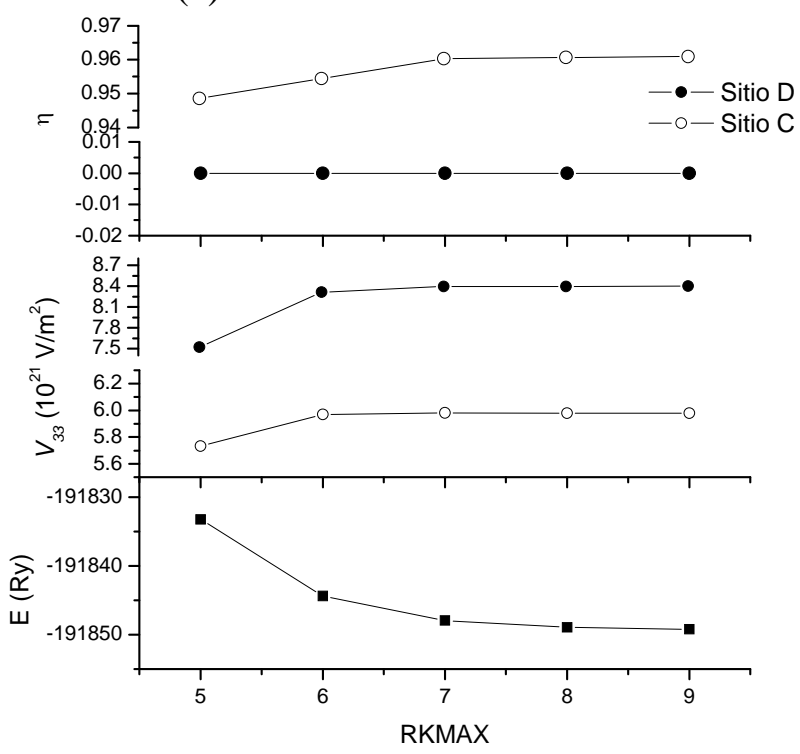

(b)

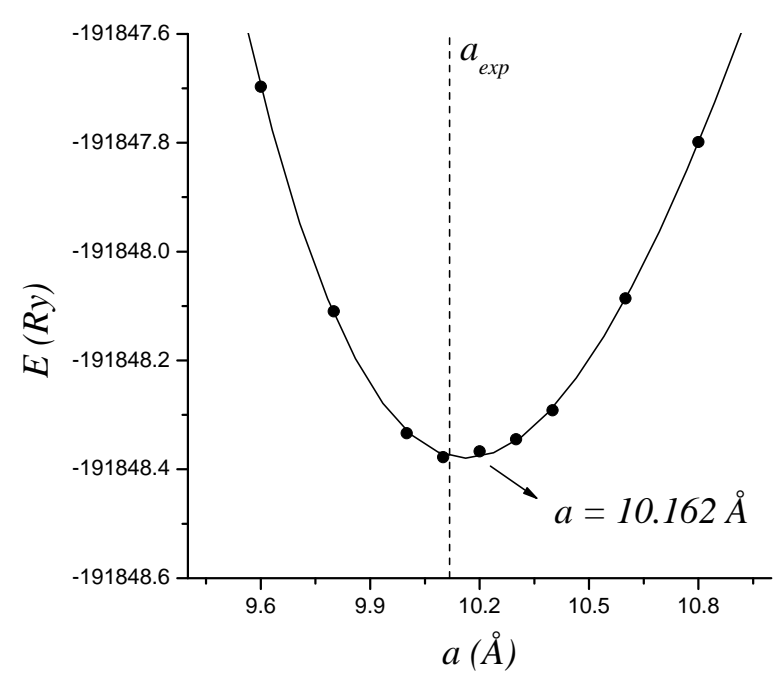

Figura 7.1: (a) Parámetros hiperfinos $\mathrm{V}_{33}$ y $\eta$ y energía de la celda de $\operatorname{In}_{2} \mathrm{O}_{3}$ en función del parámetro RKMAX. Todos los puntos corresponden a cálculos realizados con 50 puntos k en la IBZ. (b) Energía de la celda en función del parámetro de red $a$ calculada con el método FP-APW+lo en la aproximación CW-GGA. 


\begin{tabular}{cccccc}
\hline \hline & $\boldsymbol{a}(\AA)$ & $-\boldsymbol{u}$ & $\boldsymbol{x}$ & $\boldsymbol{y}$ & $\boldsymbol{z}$ \\
\hline Experimental $^{(\mathrm{a})}$ & $10.117(1)$ & $0.0332(1)$ & $0.3905(13)$ & $0.1529(11)$ & $0.3832(12)$ \\
\hline FP-APW+lo (LDA) & 10.068 & 0.0335 & 0.3901 & 0.1541 & 0.3818 \\
\hline FP-APW+lo (CW-GGA) & 10.162 & 0.0334 & 0.3900 & 0.1542 & 0.3819 \\
\hline
\end{tabular}

Tabla 7.1: Parámetro de red y parámetros estructurales internos obtenidos por medio del método FP-APW+lo para la celda de $\operatorname{In}_{2} \mathrm{O}_{3}$.

\begin{tabular}{|c|c|c|c|c|c|c|c|}
\hline \multirow{2}{*}{ Parámetros } & \multirow{2}{*}{ Aprox. } & \multicolumn{3}{|c|}{ Sitio D } & \multicolumn{3}{|c|}{ Sitio C } \\
\hline & & $\boldsymbol{d}_{N N}$ & $V_{33}$ & $\eta$ & $\boldsymbol{d}_{N N}$ & $V_{33}$ & $\eta$ \\
\hline \multirow{2}{*}{$\begin{array}{c}\text { Sin optimizar } \\
\text { (experimentales) }\end{array}$} & LDA & 2.19 & +8.42 & 0.00 & $\begin{array}{l}2.12 \\
2.19 \\
2.21 \\
\end{array}$ & +6.13 & 0.92 \\
\hline & CW-GGA & 2.19 & +8.40 & 0.00 & $\begin{array}{l}2.12 \\
2.19 \\
2.21\end{array}$ & +5.98 & 0.96 \\
\hline \multirow{2}{*}{ Optimizados } & LDA & 2.16 & +8.36 & 0.00 & $\begin{array}{l}2.12 \\
2.18 \\
2.21 \\
\end{array}$ & +5.54 & 0.94 \\
\hline & CW-GGA & 2.18 & +8.39 & 0.00 & $\begin{array}{l}2.14 \\
2.20 \\
2.23\end{array}$ & +5.66 & 0.93 \\
\hline
\end{tabular}

Tabla 7.2: Resultados FP-APW+lo para el sistema $\operatorname{In}_{2} \mathrm{O}_{3}$ utilizando los parámetros de red de la Tabla 7.1. $d_{N N}$ se expresa en $\AA$ y $V_{33}$ en unidades de $10^{21} \mathrm{~V} / \mathrm{m}^{2}$.

En la Tabla 7.2 se muestran los parámetros hiperfinos calculados en el óxido de Indio puro con parámetros de red optimizados y sin optimizar (expeirmentales). Como puede apreciarse, existe un perfecto acuerdo entre los valores de $V_{33}$ calculados con los dos grupos de parámetros para el caso del sitio D. Mientras que para la componente principal $V_{33}$ calculada en el sitio $C$ se observa una diferencia del orden de $0.5 \times 10^{21}$ $\mathrm{V} / \mathrm{m}^{2}$ entre los valores optimizados y sin optimizar en ambas aproximaciones. En el caso del parámetro de asimetría, el sitio D posee simetría axial puesto que el método de cálculo FP-APW+lo mantiene la simetría puntual del sitio catiónico en el proceso de minimización estructural. Mientras que los valores de $\eta$ del sitio C calculados en las dos aproximaciones y con los dos grupos de parámetros estructurales están en perfecto acuerdo.

(a) [Marezio 1966] 
$\mathbf{Y}_{2} \mathrm{O}_{3}$ puro: en la Figura 7.2 (a) se muestra la convergencia de los parámetros que caracterizan el GCE y de la energía de la celda de $\mathrm{Y}_{2} \mathrm{O}_{3}$, $E$, en función del parámetro RKMAX, mientras que en la Figura 7.2 (b) se grafica $E$ en función del parámetro de red a. De la convergencia de RKMAX se puede observar que todos los observables están convergidos para RKMAX=7 y el valor de $a$ que minimiza la energía del sistema es $10,562 \AA$.

En la Tabla 7.3 se muestran las coordenadas internas y el parámetro de red optimizados en ambas aproximaciones (LDA y CW-GGA) y se contrastan con sus respectivos valores experimentales. Como puede apreciarse los valores de a que minimizan la energía de la celda difieren en $0.03 \AA$ aproximadamente respecto del valor experimental, mientras que la diferencia entre las coordenadas internas optimizadas y las experimentales aparece en el tercer decimal.

En la Tabla 7.4 se comparan los parámetros hiperfinos $\left(V_{33}\right.$ y $\left.\eta\right)$ y la distancia entre el catión y sus primeros vecinos oxígeno, $d_{N N}$, calculados con la celda optimizada y con la celda experimental, esto es con parámetros estructurales optimizados y experimentales, respectivamente. Se puede observar que $d_{N N}$ difiere en menos de $0.01 \AA$ para ambos sitios. Los valores de $\mathrm{V}_{33}$ difieren en menos de $0.5 \times 10^{21} \mathrm{~V} / \mathrm{m}^{2}$ para los dos sitios. Solo en el caso del parámetro de asimetría se observa una diferencia entre 12 \% y $15 \%$ para el sitio C.

\begin{tabular}{cccccc}
\hline \hline & $\boldsymbol{a}(\AA)$ & $-\boldsymbol{u}$ & $\boldsymbol{x}$ & $\boldsymbol{y}$ & $\boldsymbol{z}$ \\
\hline Experimental $^{(\mathrm{a})}$ & $10.5989(7)$ & $0.03236(3)$ & $0.3907(2)$ & $0.1518(2)$ & $0.3801(2)$ \\
\hline FP-APW+lo (LDA) & 10.560 & 0.03299 & 0.3901 & 0.1508 & 0.3799 \\
\hline FP-APW+lo (CW - GGA) & 10.562 & 0.03290 & 0.3906 & 0.1514 & 0.3796 \\
\hline \hline
\end{tabular}

Tabla 7.3: Parámetro de red y parámetros estructurales internos obtenidos por medio del método FP-APW+lo para la celda de $\mathrm{Y}_{2} \mathrm{O}_{3}$.

(a) [Maslen 1996] 
(a)

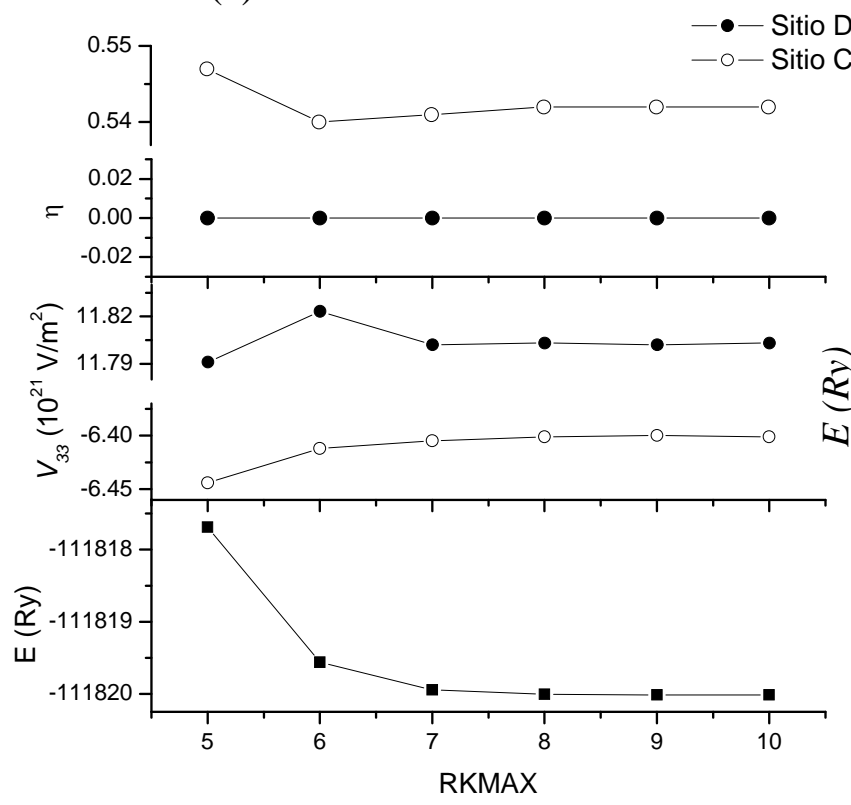

(b)

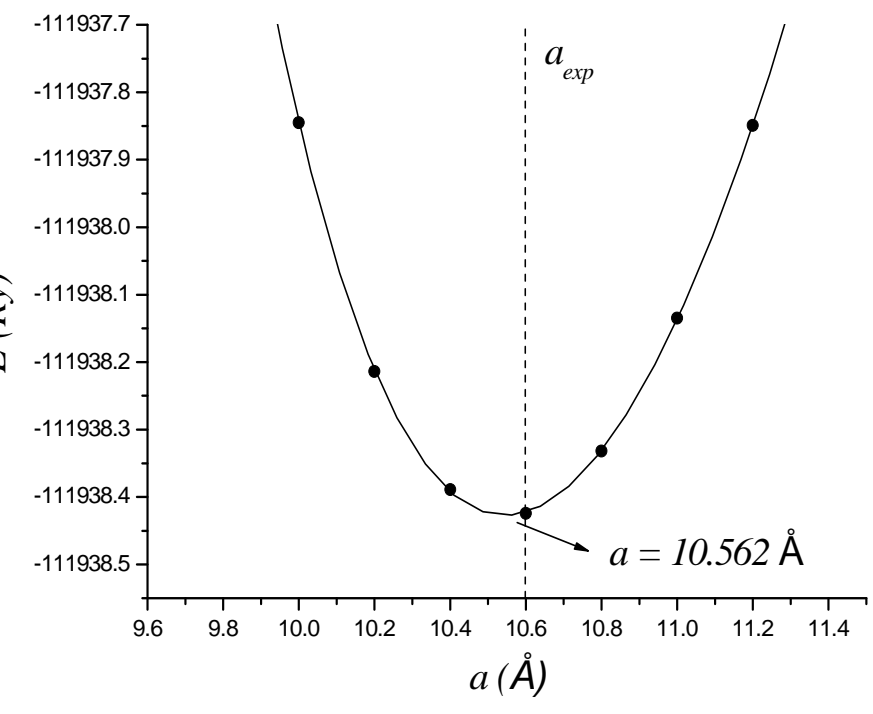

Figura 7.2: (a) Parámetros hiperfinos $\mathrm{V}_{33}$ y $\eta$ y energía de la celda de $\mathrm{Y}_{2} \mathrm{O}_{3}$ en función del parámetro RKMAX. Todos los puntos corresponden a cálculos realizados con 50 puntos $\mathrm{k}$ en la IBZ. (b) Energía de la celda en función del parámetro de red $a$ calculada con el método FP-APW+lo en la aproximación CW-GGA.

\begin{tabular}{|c|c|c|c|c|c|c|c|}
\hline \multirow{2}{*}{ Parámetros } & \multirow{2}{*}{ Aprox. } & \multicolumn{3}{|c|}{ Sitio D } & \multicolumn{3}{|c|}{ Sitio C } \\
\hline & & $\boldsymbol{d}_{N N}$ & $V_{33}$ & $\eta$ & $d_{N N}$ & $V_{33}$ & $\eta$ \\
\hline \multirow{6}{*}{$\begin{array}{c}\text { Sin optimizar } \\
\text { (experimentales) }\end{array}$} & & & & & 2.24 & & \\
\hline & LDA & 2.28 & +11.80 & 0.00 & 2.27 & -6.40 & 0.54 \\
\hline & & & & & 2.33 & & \\
\hline & & & & & 2.24 & & \\
\hline & CW-GGA & 2.28 & +11.72 & 0.00 & 2.27 & -6.39 & 0.53 \\
\hline & & & & & 2.33 & & \\
\hline \multirow{6}{*}{ Optimizados } & & & & & 2.24 & & \\
\hline & LDA & 2.27 & +12.39 & 0.00 & 2.25 & -6.57 & 0.39 \\
\hline & & & & & 2.31 & & \\
\hline & & & & & 2.24 & & \\
\hline & CW-GGA & 2.27 & +12.25 & 0.00 & 2.26 & -6.34 & 0.41 \\
\hline & & & & & 2.32 & & \\
\hline
\end{tabular}

Tabla 7.4: Resultados FP-APW+lo para el sistema $\mathrm{Y}_{2} \mathrm{O}_{3}$ utilizando los parámetros de red de la Tabla 7.3. $d_{N N}$ se expresa en $\AA$ y $V_{33}$ en unidades de $10^{21} \mathrm{~V} / \mathrm{m}^{2}$.

$\boldsymbol{S}_{2} \boldsymbol{O}_{3}$ puro: en la Figura 7.3 (a) se muestra la convergencia de $V_{33}, \eta$ y $E$ en función del parámetro $R K M A X$ y en la Figura 7.3 (b) se representa la energía de la celda en función del parámetro de red. Al igual que en el caso de las otras bixbitas, puede apreciarse que 
los tres parámetros están convergidos a partir de $R K M A X=7$. El valor de $a$ que minimiza la energía de la celda de $\mathrm{Sc}_{2} \mathrm{O}_{3}$ es $9.798 \AA$.

En la Tabla 7.5 se muestran las parámetros estructurales internos y el parámetro de red optimizados con las aproximaciones LDA y CW-GGA y se los compara con sus respectivos valores experimentales. El valor de $a$ en la aproximación LDA difiere en $0.15 \AA$ aproximadamente de su valor experimental, mientras que $a$ en la aproximación CW-GGA presenta una diferencia menor a $0.5 \AA$ respecto al experimento. Cabe destacar que en todas las bixbitas se observa que el valor optimizado de $a$ en la aproximación LDA es menor al optimizado mediante CW-GGA. Respecto a las coordenadas internas $u, x, y, z$, en general la diferencia respecto a sus valores experimentales está en el tercer o cuarto decimal.

Finalmente, en la Tabla 7.6 se muestran los valores de $V_{33}, \eta$ y $d_{N N}$ calculados con la celda optimizada y con la celda experimental. El valor $d_{N N}$ optimizado correspondiente al sitio C difiere en menos de $0.02 \AA$ respecto a su valor experimental, mientras que en el caso del sitio D la diferencia es $0.01 \AA$ o menor. Respecto a la componente principal $\mathrm{V}_{33}$, la diferencia en todos los casos (sitios $\mathrm{C}$ y D) siempre es menor a $0.2 \times 10^{21} \mathrm{~V} / \mathrm{m}^{2}$. El valor de $\eta$ es el que presenta mayor diferencia, la cual es de 19 \% para LDA y $17 \%$ para CW-GGA.

(a)

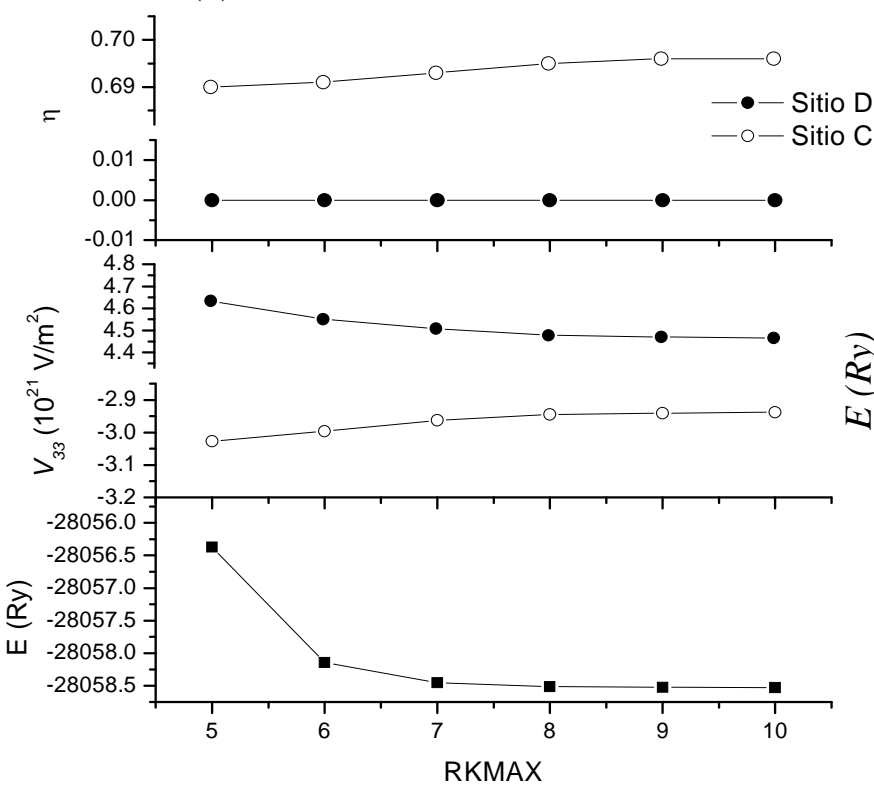

(b)

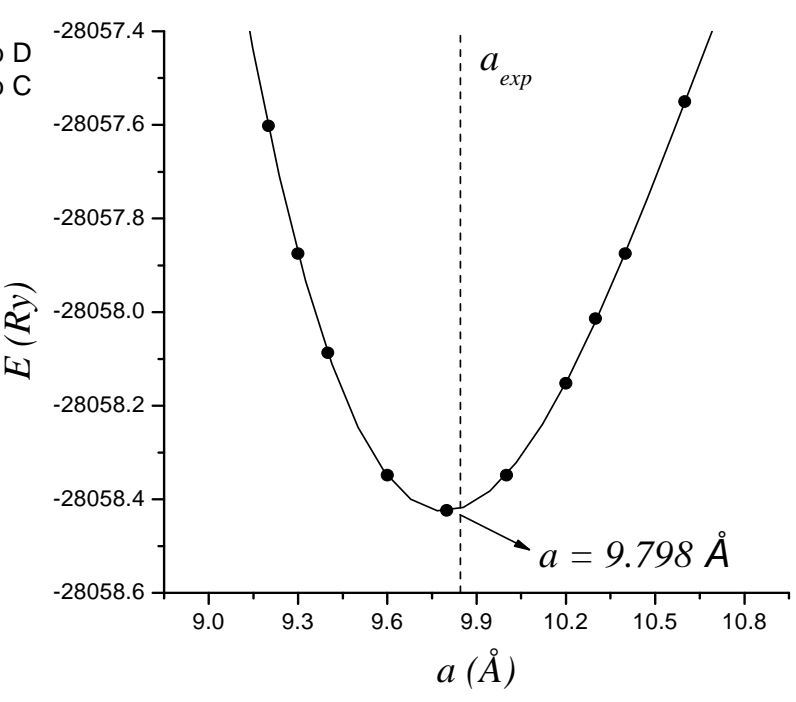

Figura 7.3: (a) Parámetros hiperfinos $\mathrm{V}_{33}$ y $\eta$ y energía de la celda de $\mathrm{Sc}_{2} \mathrm{O}_{3}$ en función del parámetro RKMAX. Todos los puntos corresponden a cálculos realizados con 50 puntos $\mathrm{k}$ en la IZB. (b) Energía de la celda en función del parámetro de red $a$ calculada con el método FP-APW+lo en la aproximación CW-GGA. 


\begin{tabular}{cccccc}
\hline \hline & $\boldsymbol{a}(\AA)$ & $-\boldsymbol{u}$ & $\boldsymbol{x}$ & $\boldsymbol{y}$ & $\boldsymbol{z}$ \\
\hline Experimental $^{(\mathrm{a})}$ & 9.845 & 0.03546 & 0.39137 & 0.15477 & 0.38137 \\
\hline FP-APW+lo (LDA) & 9.708 & 0.0364 & 0.3915 & 0.1545 & 0.3810 \\
\hline FP-APW+lo (CW - GGA) & 9.798 & 0.0361 & 0.3913 & 0.1543 & 0.3812 \\
\hline
\end{tabular}

Tabla 7.5: Parámetros de red y estructurales internos obtenidos por medio del método FP-APW+lo para la celda de $\mathrm{Sc}_{2} \mathrm{O}_{3}$.

\begin{tabular}{|c|c|c|c|c|c|c|c|}
\hline \multirow{2}{*}{ Parámetros } & \multirow{2}{*}{ Aprox. } & \multicolumn{3}{|c|}{ Sitio D } & \multicolumn{3}{|c|}{ Sitio C } \\
\hline & & $\boldsymbol{d}_{N N}$ & $V_{33}$ & $\eta$ & $\boldsymbol{d}_{N N}$ & $V_{33}$ & $\eta$ \\
\hline \multirow{2}{*}{$\begin{array}{c}\text { Sin optimizar } \\
\text { (experimentales) }\end{array}$} & LDA & 2.12 & +4.53 & 0.00 & $\begin{array}{l}2.08 \\
2.12 \\
2.16\end{array}$ & -2.98 & 0.71 \\
\hline & CW-GGA & 2.12 & +4.52 & 0.00 & $\begin{array}{l}2.08 \\
2.12 \\
2.16 \\
\end{array}$ & -2.97 & 0.69 \\
\hline \multirow{2}{*}{ Optimizados } & LDA & 2.12 & +4.80 & 0.00 & $\begin{array}{l}2.07 \\
2.10 \\
2.13 \\
\end{array}$ & -3.02 & 0.52 \\
\hline & CW-GGA & 2.11 & +4.69 & 0.00 & $\begin{array}{l}2.08 \\
2.11 \\
2.15\end{array}$ & -2.90 & 0.52 \\
\hline
\end{tabular}

Tabla 7.6: Resultados FP-APW+lo para el sistema $\mathrm{Sc}_{2} \mathrm{O}_{3}$ utilizando los parámetros de red de la Tabla 7.5. $d_{N N}$ se expresa en $\AA$ y $V_{33}$ en unidades de $10^{21} \mathrm{~V} / \mathrm{m}^{2}$.

\section{7-1-2 Cálculos en ZnO puro}

La convergencia de los parámetros hiperfinos $V_{33}$ y $\eta$ y de la energía de la celda de ZnO en función de RKMAX se muestran en la Figura 7.4 (a) mientras que en la Figura 7.4 (b) se representa la superficie de energía en función de los parámetros de red $a$ y $c$. Al igual que en el caso de las bixbitas, los observables están convergidos para $R K M A X=7$. Por otro lado, el mínimo de la superficie de energía fue encontrado en el punto determinado por $a=3.2459 \AA$ y $c=5.2378 \AA$.

(a) [Norrestam 1968] 
(a)

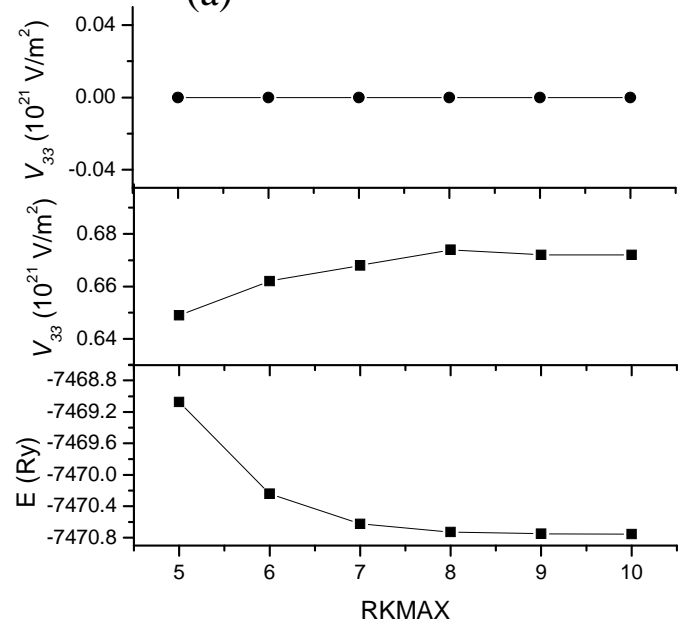

(b)

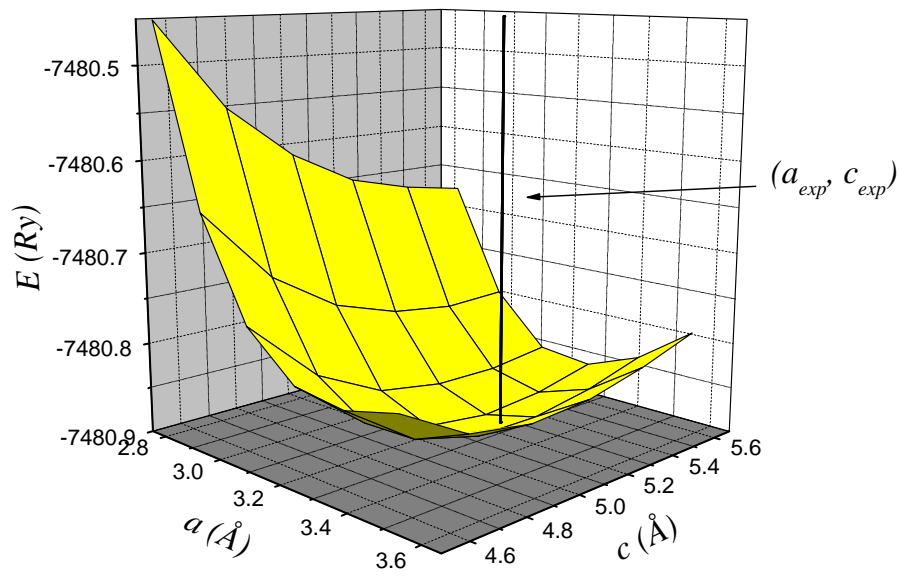

Figura 7.4: (a) Parámetros hiperfinos $\mathrm{V}_{33}$ y $\eta$ y energía de la celda de $\mathrm{ZnO}$ en función del parámetro RKMAX. Todos los puntos corresponden a cálculos realizados con 50 puntos k en la IZB. (b) Energía de la celda en función de los parámetros de red $a$ y $c$ calculada con el método FP-APW+lo en la aproximación CW-GGA.

En la Tabla 7.7 se muestra la optimización de los parámetros de red y de la coordenada interna que caracteriza las posiciones de los oxígenos. De la comparación entre los parámetros de red optimizados y los experimentales se aprecia que difieren en menos de $0.05 \AA$ en el caso de $a(=b)$ por defecto en ambos métodos, y en menos de $0.07 \AA$ en el caso de $c$ por exceso en la aproximación CW-GGA y por defecto para LDA. Y por último, la diferencia en el parámetro estructural $u$ aparece en el tercer decimal.

En la Tabla 7.8 se detallan los observables $d_{N N}, V_{33}$ y $\eta$ calculados con ambas aproximaciones para el caso de la celda determinada por coordenadas experimentales y para la celda obtenida con coordenadas optimizadas. Como puede apreciarse las distancias calculadas con coordenadas optimizadas difieren en menos de $0.03 \AA$ de las experimentales. Respecto a la componente principal $V_{33}$, se aprecia una diferencia de $0.13 \times 10^{21} \mathrm{~V} / \mathrm{m}^{2}$ para LDA y de $0.25 \times 10^{21} \mathrm{~V} / \mathrm{m}^{2}$ para CW-GGA. Finalmente, el parámetro $\eta$ es nulo en todos los casos debido a que el método de cálculo mantiene la simetría axial (respecto del eje $c$ ) del sitio catiónico. 


\begin{tabular}{ccccc}
\hline & $\boldsymbol{a}(\AA)$ & $\boldsymbol{b}(\AA)$ & $\boldsymbol{c}(\AA)$ & $\boldsymbol{u}$ \\
\hline Experimental $^{(\mathrm{a})}$ & $3.2501(1)$ & $3.2501(1)$ & $5.2071(1)$ & $0.3817(3)$ \\
\hline FP-APW+lo - LDA & 3.2083 & 3.2083 & 5.1308 & 0.3814 \\
\hline FP-APW+lo - CW - GGA & 3.2459 & 3.2459 & 5.2378 & 0.3805 \\
\hline \hline
\end{tabular}

Tabla 7.7: Parámetros de red y estructurales internos obtenidos con el método FPAPW+lo para la celda de $\mathrm{ZnO}$.

\begin{tabular}{ccccc}
\hline \hline Parámetros & Aprox. & $\boldsymbol{d}_{\boldsymbol{N}}(\AA)$ & $\boldsymbol{\eta}$ & $\boldsymbol{V}_{\mathbf{3 3}}\left(\mathbf{1 0 ^ { 2 1 } \mathbf { V } / \mathbf { m } ^ { 2 } )}\right.$ \\
\hline \multirow{2}{*}{$\begin{array}{c}\text { Sin optimizar } \\
\text { (experimentales) }\end{array}$} & LDA & $\begin{array}{l}1.97 \\
1.99\end{array}$ & 0.000 & 0.68 \\
\cline { 2 - 5 } & CW-GGA & $\begin{array}{l}1.97 \\
1.99\end{array}$ & 0.000 & 0.67 \\
\hline \multirow{2}{*}{ Optimizados } & LDA & $\begin{array}{l}1.95 \\
1.96\end{array}$ & 0.000 & 0.54 \\
\cline { 2 - 5 } & CW-GGA & $\begin{array}{l}1.98 \\
1.98\end{array}$ & 0.000 & 0.41 \\
\hline \hline
\end{tabular}

Tabla 7.8: Resultados FP-APW+lo para el sistema ZnO utilizando los parámetros de red de la Tabla 7.7. $d_{N N}$ se expresa en $\AA$ y $V_{33}$ en unidades de $10^{21} \mathrm{~V} / \mathrm{m}^{2}$.

\section{7-1-3 Cálculos en $\mathrm{SnO}_{2}$ puro}

La convergencia del parámetro $R K M A X$ para el sistema $\mathrm{SnO}_{2}$ se muestra en la Figura 7.5 (a), mientras que la minimización de la energía de la celda en función del parámetro de red se esquematiza en la Figura 7.5 (b). Al igual que en el caso de los otros óxidos, la convergencia es alcanzada a partir de $R K M A X=7$. El mínimo energético fue encontrado en los parámetros $a=4.7710 \AA$ y $c=3.1999 \AA$.

\footnotetext{
(a) [Kisi 1989]
} 
(a)

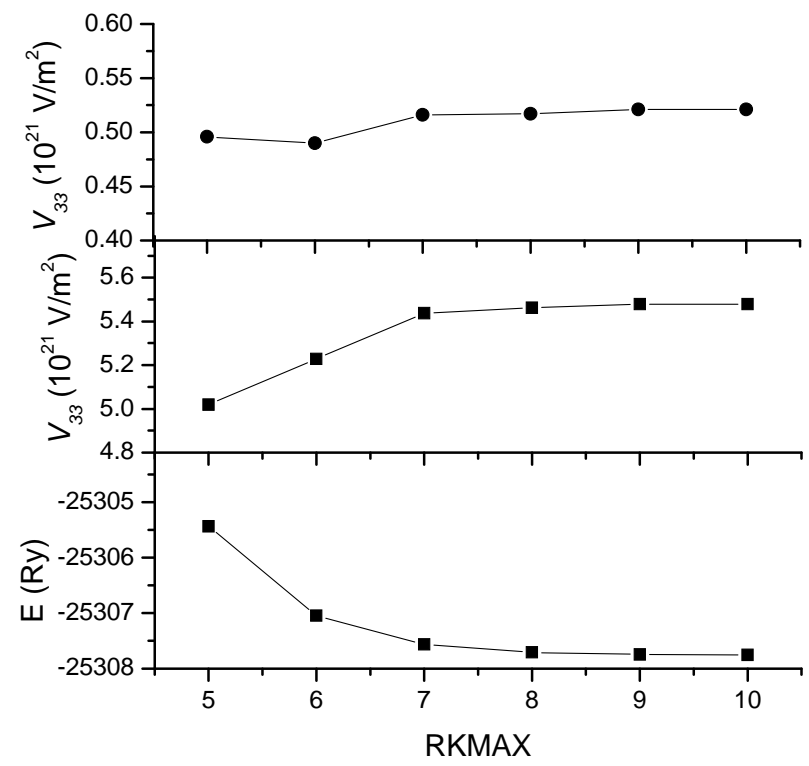

(b)

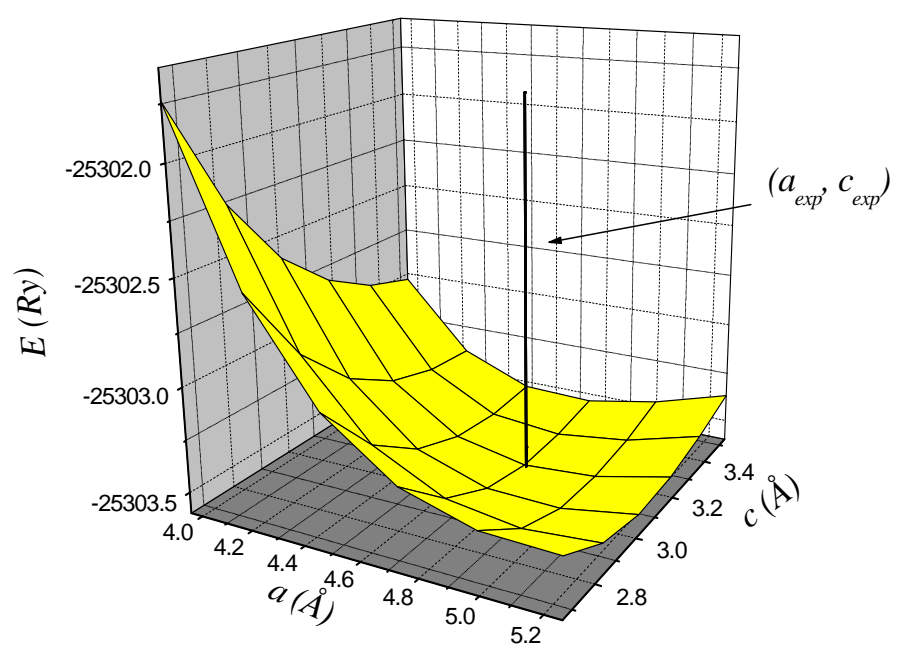

Figura 7.5: (a) Parámetros hiperfinos $\mathrm{V}_{33}$ y $\eta$ y energía de la celda de $\mathrm{SnO}_{2}$ en función del parámetro RKMAX, Todos los puntos corresponden a cálculos realizados con 50 puntos k en la IZB. (b) Energía de la celda en función de los parámetros de red $a$ y $c$ calculada con el método FP-APW+lo en la aproximación CW-GGA.

\begin{tabular}{ccccc}
\hline \hline & $\boldsymbol{a}(\AA)$ & $\boldsymbol{b}(\AA)$ & $\boldsymbol{c}(\AA)$ & $\boldsymbol{u}$ \\
\hline Experimental $^{(\mathbf{a})}$ & $4.7374(1)$ & $4.7374(1)$ & 3.1864 & $0.3056(1)$ \\
\hline FP-APW+lo - LDA & 4.7297 & 4.7297 & 3.1995 & 0.3061 \\
\hline FP-APW+lo - CW - GGA & 4.7710 & 4.7710 & 3.1999 & 0.3056 \\
\hline \hline
\end{tabular}

Tabla 7.9: Parámetro de red y parámetros estructurales internos obtenidos por medio del método FP-APW+lo para la celda de $\mathrm{SnO}_{2}$. 


\begin{tabular}{|c|c|c|c|c|}
\hline Parámetros & Aprox. & $d_{N N}(\AA)$ & $\eta$ & $V_{33}\left(10^{21} \mathrm{~V} / \mathrm{m}^{2}\right)$ \\
\hline \multirow{2}{*}{$\begin{array}{c}\text { Sin optimizar } \\
\text { (experimentales) }\end{array}$} & LDA & $\begin{array}{l}2.05 \\
2.06\end{array}$ & 0.49 & +5.06 \\
\hline & CW-GGA & $\begin{array}{l}2.05 \\
2.06\end{array}$ & 0.52 & +5.44 \\
\hline \multirow{2}{*}{ Optimizados } & LDA & $\begin{array}{l}2.05 \\
2.06\end{array}$ & 0.44 & +5.71 \\
\hline & CW-GGA & $\begin{array}{l}2.06 \\
2.07\end{array}$ & 0.68 & +5.10 \\
\hline
\end{tabular}

Tabla 7.10: Resultados FP-APW+lo para el sistema $\mathrm{SnO}_{2}$ utilizando los parámetros de red de la Tabla 7.7. $d_{N N}$ se expresa en $\AA$ y $V_{33}$ en unidades de $10^{21} \mathrm{~V} / \mathrm{m}^{2}$.

En la Tabla 7.9 se muestra los parámetros de red optimizados y sin optimizar para las aproximaciones LDA y CW-GGA del método FP-APW+lo. De la comparación entre los parámetros optimizados y experimentales se aprecia una diferencia en menos de $0.05 \AA$ en el caso de $a(=b)$ por defecto en la aproximación LDA y por exceso en CWGGA mientras que en el caso de $c$ por exceso en ambas aproximaciones. Y por último, la diferencia en el parámetro estructural $u$ aparece en el tercer decimal en LDA mientras que para CW-GGA el parámetro no sufrió variaciones.

En la Tabla 7.10 se comparan los observables $d_{N N}, V_{33}$ y $\eta$ calculados para el caso de la celda calculada con coordenadas experimentales y con coordenadas optimizadas en ambas aproximaciones. Como puede apreciarse las distancias calculadas con coordenadas optimizadas difieren en menos de $0.02 \AA$ de las experimentales. Respecto a la componente principal $\mathrm{V}_{33}$, se aprecia una diferencia de $0.7 \times 10^{21} \mathrm{~V} / \mathrm{m}^{2}$ para LDA y de $0.4 \times 10^{21} \mathrm{~V} / \mathrm{m}^{2}$ para CW-GGA. Finalmente, para el parámetro $\eta$ se observa una diferencia del 5\% para el caso de LDA y del 17\% para CW-GGA.

\section{7-1-4 Cálculos en SnO puro}

La convergencia de $E, V_{33}$ y $\eta$ en función del parámetro $R K M A X$ para el sistema SnO se muestra en la Figura 7.6 (a), mientras que la superficie de energía en función de los parámetros de red $a$ y $c$ se esquematiza en la Figura 7.6 (b). A partir de RKMAX=7 se aprecia la convergencia de los tres observables. El mínimo de la superficie de energía fue encontrado para los parámetros $a=3.7966 \AA$ y $c=4.7673 \AA$. 
En la Tabla 7.11 se muestra los parámetros de red optimizados y experimentales para ambas aproximaciones del método FP-APW+lo. De la comparación entre los parámetros de red optimizados y los experimentales se aprecia que difieren en menos de $0.05 \AA \AA$ en el caso de $a(=b)$ por defecto en ambas aproximaciones, mientras que en el caso $c$ la diferencia es menor que $0.07 \AA$ también por defecto en las dos aproximaciones. Y por último, la diferencia en el parámetro estructural $u$ aparece en el tercer decimal tanto para LDA como para CW-GGA.

(a)

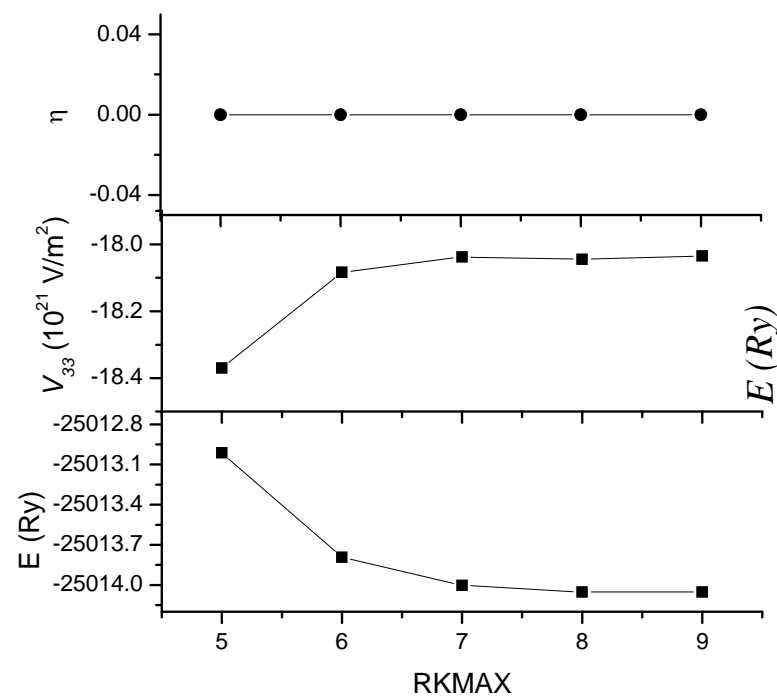

(b)

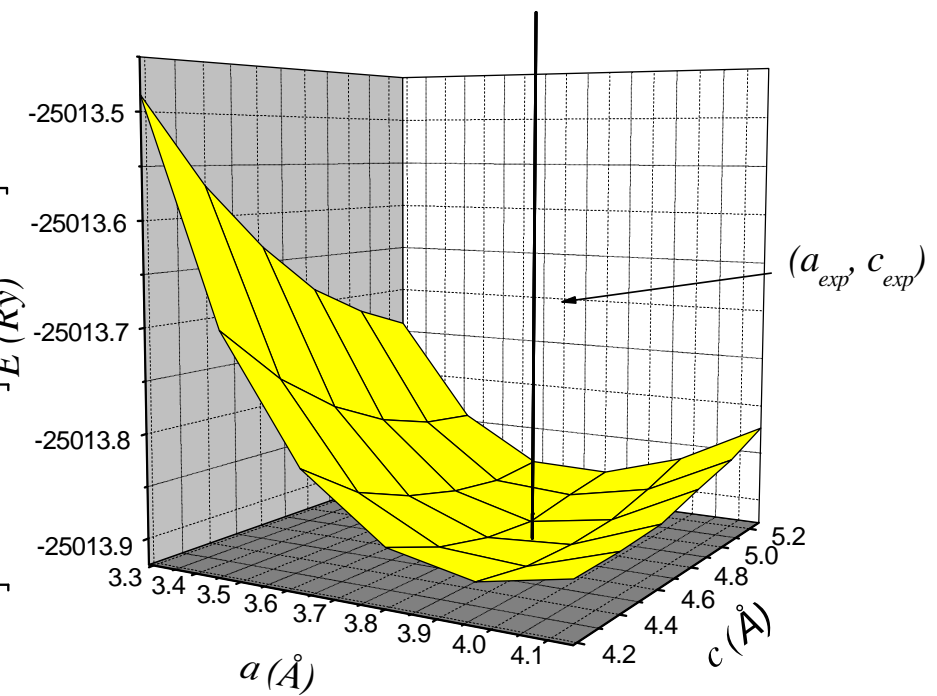

Figura 7.6: (a) Parámetros hiperfinos $V_{33}$ y $\eta$ y energía de la celda de $\mathrm{SnO}$ en función del parámetro RKMAX. Todos los puntos corresponden a cálculos realizados con 50 puntos $\mathrm{k}$ en la IZB. (b) Energía de la celda en función de los parámetros de red $a$ y $c$ calculada con el método FP-APW+lo en la aproximación CW-GGA.

\begin{tabular}{ccccc}
\hline \hline & $\boldsymbol{a}(\AA)$ & $\boldsymbol{b}(\AA)$ & $\boldsymbol{c}(\AA)$ & $\boldsymbol{u}$ \\
\hline Experimental $^{(\mathbf{a})}$ & $3.8029(5)$ & $3.8029(5)$ & 4.8382 & 0.2369 \\
\hline FP-APW+lo - LDA & 3.7564 & 3.7564 & 4.7621 & 0.2409 \\
\hline FP-APW+lo - CW - GGA & 3.7966 & 3.7966 & 4.7673 & 0.2373 \\
\hline \hline
\end{tabular}

Tabla 7.11: Parámetro de red y parámetros estructurales internos obtenidos por medio del método FP-APW+lo para la celda de SnO.

\footnotetext{
(a) [Wang 2004]
} 


\begin{tabular}{|c|c|c|c|c|}
\hline Parámetros & Aprox. & $d_{N N}(\AA)$ & $\eta$ & $V_{33}\left(10^{21} \mathrm{~V} / \mathrm{m}^{2}\right)$ \\
\hline \multirow{2}{*}{ Sin optimizar } & LDA & 2.22 & 0.00 & -18.16 \\
\hline & CW-GGA & 2.22 & 0.00 & -18.04 \\
\hline \multirow{2}{*}{ Optimizados } & LDA & 2.20 & 0.00 & -19.05 \\
\hline & CW-GGA & 2.22 & 0.00 & -18.22 \\
\hline
\end{tabular}

Tabla 7.12: Resultados FP-APW+lo para el sistema SnO utilizando los parámetros de red de la Tabla 7.7. $d_{N N}$ se expresa en $\AA$ y $V_{33}$ en unidades de $10^{21} \mathrm{~V} / \mathrm{m}^{2}$.

En la Tabla 7.12 se comparan los observables $d_{N N}, V_{33}$ y $\eta$ calculados para el caso de la celda calculada con coordenadas experimentales y con coordenadas optimizadas en ambas aproximaciones. La distancia entre el catión y los primeros vecinos se ve modificada en 0.019 Å para LDA y en 0.003 Å para CW-GGA. El parámetro $\eta$ es nulo en todos los casos debido a que el método de cálculo mantiene la simetría axial (respecto del eje $c$ ) del sitio catiónico durante el proceso de relajación estructural. Mientras que en el caso de $\mathrm{V}_{33}$, los valores optimizados y sin optimizar difieren en $0.9 * 10^{21} \mathrm{~V} / \mathrm{m}^{2}$ para LDA y en $0.2 * 10^{21} \mathrm{~V} / \mathrm{m}^{2}$ para CW-GGA.

\section{7-2 Cálculos $a b$ initio en sistemas dopados}

En este apartado se presentan los cálculos FP-APW+lo para el caso de los sistemas dopados. Primero se muestran cálculos en el óxido $\mathrm{Sc}_{2} \mathrm{O}_{3}$ dopado con Cd realizados con la celda no relajada y con la celda relajada. En este último caso, tal como se explicó en la sección 2-3 del Capítulo 4, se permite el desplazamiento de cada átomo de la supercelda manteniendo la simetría puntual del sistema hasta que las fuerzas cuánticas sobre cada átomo no superen un valor de tolerancia (en nuestro caso es de $0.025 \mathrm{eV} / \AA ̊$ ). Tales relajaciones se deben a la introducción del átomo Cd en la red huésped, la cual introduce distorsiones estructurales y electrónicas debido a su carácter de impureza.

\section{7-2-1 Comparación de cálculos en $\mathrm{Sc}_{2} \mathrm{O}_{3}$ :Cd con celda relajada y no relajada}

Para demostrar la necesidad de considerar las relajaciones estructurales en el estudio de óxidos semiconductores dopados con impurezas analizaremos en particular el caso de óxido de Escandio dopado con Cd. Cabe destacar que a partir del estudio de la convergencia del tensor GCE y de la energía total de la supercelda con el parámetro 
RKMAX y el número de puntos k en el espacio recíproco, concluimos que con un valor de 7 para RKMAX y con 50 puntos k en la IZB del espacio recíproco los parámetros hiperfinos calculados en los sistemas puros se encuentran convergidos.

En la Tabla 7.13 comparamos el parámetro de asimetría $\eta$, la componente principal del GCE $V_{33}$ y su dirección para el caso de celda sin relajaciones y celda relajada con los resultados PAC obtenidos en sitios del átomo-impureza ${ }^{111} \mathrm{Cd}$ difundido en muestras policristalinas de $\mathrm{Sc}_{2} \mathrm{O}_{3}$.

\begin{tabular}{|c|c|c|c|c|c|c|c|c|c|}
\hline \multirow{2}{*}{ Métodología } & \multirow{2}{*}{$\begin{array}{l}\text { Tipo de } \\
\text { celda }\end{array}$} & \multicolumn{4}{|c|}{ Sitio D } & \multicolumn{4}{|c|}{ Sitio C } \\
\hline & & $\boldsymbol{d}_{N N}$ & $V_{33}$ & $\eta$ & Dir. $V_{33}$ & $\boldsymbol{d}_{N N}$ & $V_{33}$ & $\eta$ & Dir. $V_{33}$ \\
\hline \multirow{2}{*}{$\begin{array}{l}\text { APW+lo- } \\
\text { CW GGA }\end{array}$} & $\begin{array}{c}\text { No } \\
\text { relajada }\end{array}$ & 2.12 & 8.96 & 0.00 & {$\left[\begin{array}{lll}1 & 1 & 1\end{array}\right]$} & $\begin{array}{l}2.08 \\
2.12 \\
2.16\end{array}$ & -5.02 & 0.65 & [1 $1-0.8$ 0 $]$ \\
\hline & Relajada & 2.28 & 8.16 & 0.00 & {$\left[\begin{array}{lll}1 & 1 & 1\end{array}\right]$} & $\begin{array}{l}2.14 \\
2.28 \\
2.32 \\
\end{array}$ & 6.46 & 0.73 & {$\left[\begin{array}{lll}0.8 & 1 & 0\end{array}\right]$} \\
\hline Experimental & - & - & $8.22(1)$ & 0.00 & {$\left[\begin{array}{lll}1 & 1 & 1\end{array}\right]$} & - & $6.56(1)$ & $0.70(1)$ & - \\
\hline
\end{tabular}

Tabla 7.13: Comparación de cálculos APW+lo de $d_{N N}, \mathrm{~V}_{33}$ (dirección y magnitud) y $\eta$ en la celda relajada y sin relajaciones con resultados PAC para el caso de $\mathrm{Sc}_{2} \mathrm{O}_{3}:{ }^{111} \mathrm{Cd}$.

Como puede apreciarse en la tabla, para poder reproducir las medidas experimentales del GCE en ${ }^{111} \mathrm{Cd}$ localizado en el sitio $\mathrm{C}$ del óxido $\mathrm{Sc}_{2} \mathrm{O}_{3}$ es imprescindible considerar las relajaciones estructurales predichas por el cálculo $a b$ initio. Como se puede observar, la componente principal del GCE cambia en signo, dirección y magnitud al tener en cuenta las relajaciones. En particular, la magnitud de $\mathrm{V}_{33}$ presenta un cambio aproximado del $30 \%$.

En cambio, para el caso de ${ }^{111} \mathrm{Cd}$ localizado en sitio D, la discrepancia entre la componente $\mathrm{V}_{33}$ calculada con celda no relajada y la medida PAC no supera el $10 \%$. Esto se atribuye a la alta simetría que presenta el sitio D, por lo que solo se observa una disminución de $\mathrm{V}_{33}$ al considerar las relajaciones en acuerdo con la dependencia con $r^{-3}$ del GCE respecto a la carga que lo produce.

Teniendo en cuenta que para reproducir correctamente los parámetros hiperfinos medidos por la técnica $\mathrm{PAC}$ en $\mathrm{Sc}_{2} \mathrm{O}_{3}$ en el sitio de la sonda ${ }^{111} \mathrm{Cd}$ es necesario considerar las distorsiones estructurales introducidas por la impureza en la red huésped, 
entonces, en los cálculos $a b$ initio realizados en todos los sistemas dopados en el marco del presente trabajo se han tenido en cuenta las relajaciones estructurales producidas por el Cd en el sistema dopado.

En la siguiente sección estudiaremos la convergencia en RKMAX de los parámetros hiperfinos calculados en los sistemas dopados para el caso de celda relajada.

\section{7-2-2 Estudio de la convergencia de parámetros hiperfinos en los sistemas dopados}

Primero analizaremos la convergencia del parámetro $\eta$, de la compenente $V_{33}$ y de la energía total de la supercelda como una función del parámetro de cálculo RKMAX en los óxidos con estructura bixbita. En la Figura 7.7 se aprecia que en el caso de las bixbitas tanto la energía del sistema como el tensor GCE están convergidos para RKMAX $=7$.

(a)

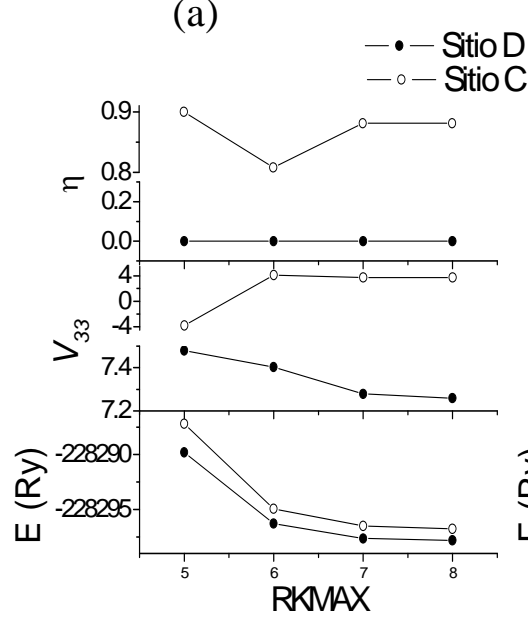

(b)

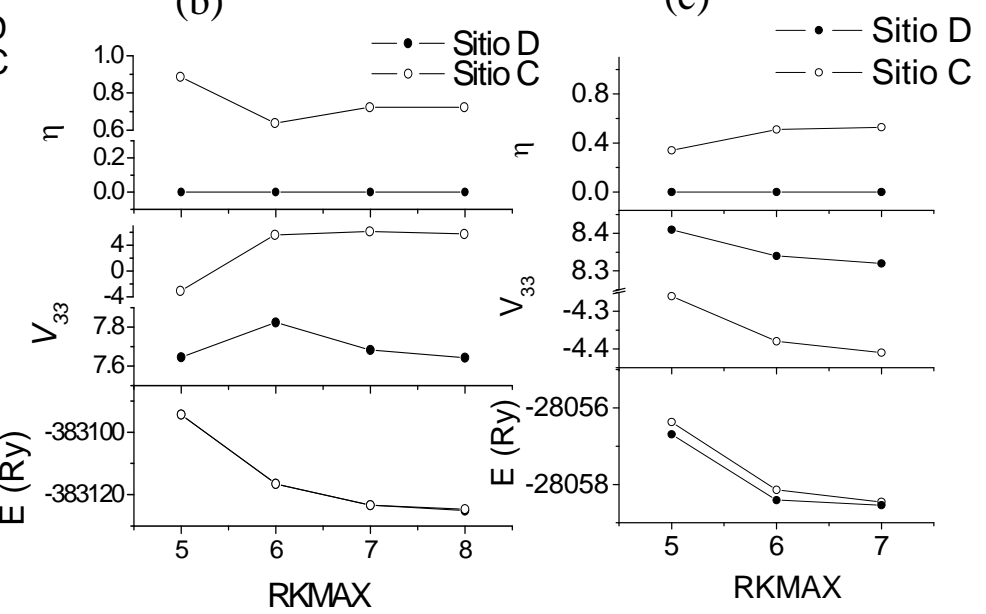

Figura 7.7: Parámetros hiperfinos $\eta, \mathrm{V}_{33}$ y la energía del sistema vs. RKMAX para el caso de las siguientes bixbitas: (a) $\mathrm{In}_{2} \mathrm{O}_{3}$ :Cd, (b) $\mathrm{Y}_{2} \mathrm{O}_{3}: C d$, (c) $\mathrm{Sc}_{2} \mathrm{O}_{3}$ :Cd. $\mathrm{V}_{33}$ viene dado en unidades de $10^{21} \mathrm{~V} / \mathrm{m}^{2}$.

En la Figura 7.8 se representan los parámetros que caracterizan el GCE y la energía del sistema dopado con $\mathrm{Cd}$ para el caso del $\mathrm{ZnO}, \mathrm{SnO}_{2}$ y $\mathrm{SnO}$. Como puede apreciarse en todos los casos el GCE y la energía de la supercelda están convergidas también para el valor 7 del parámetro RKMAX.

Para terminar de analizar la convergencia estudiaremos el comportamiento de la distancia entre la impureza $\mathrm{Cd}$ y los átomos $\mathrm{O}_{\mathrm{NN}}$ en función de RKMAX. En la Figura 7.9 se muestra la distancia $d_{C d-O N N}$ vs. RKMAX para el caso de las bixbitas y en la Figura 7.10 los óxidos $\mathrm{ZnO}, \mathrm{SnO}_{2}$ y $\mathrm{SnO}$. 

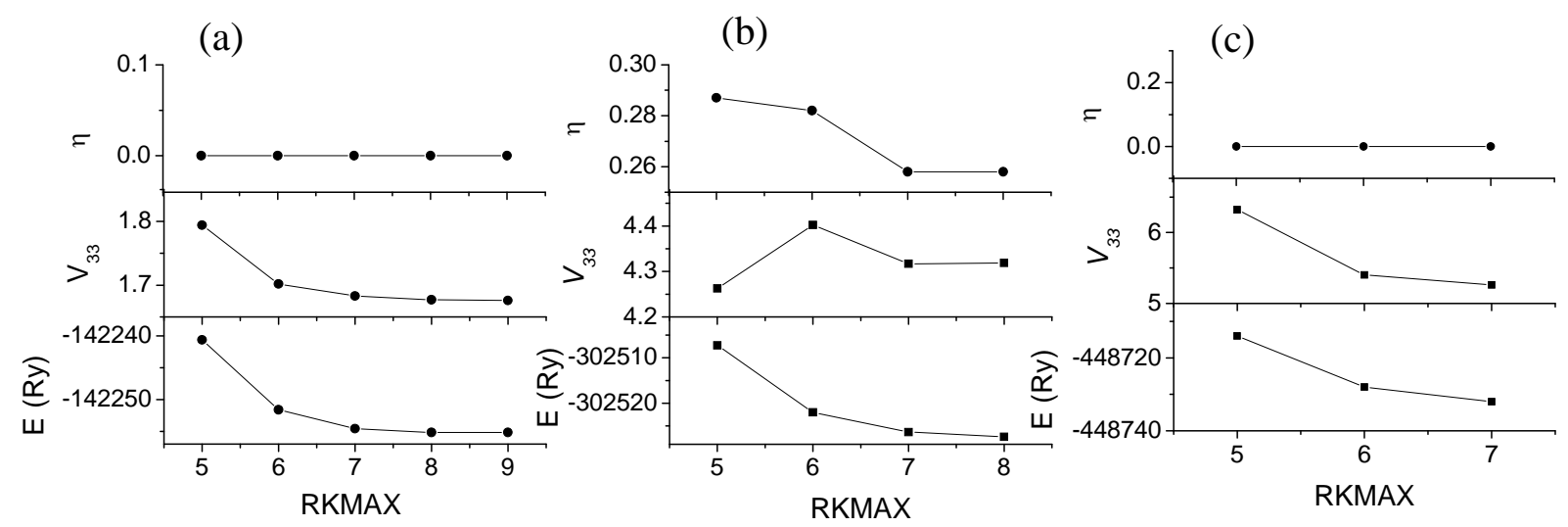

Figura 7.8: Parámetros $\eta, V_{33}$ y la energía total $E$ para los óxidos: (a) ZnO:Cd, (b) $\mathrm{SnO}_{2}$ :Cd y (c) SnO:Cd. $\mathrm{V}_{33}$ viene dado en unidades de $10^{21} \mathrm{~V} / \mathrm{m}^{2}$.

Como puede apreciarse en la Figura 7.9 las distancias $d_{C d-O N N}$ están convergidas para $\mathrm{RKMAX}=7$ para el caso de todas las bixbitas, mientras que lo mismo se aprecia en la Figura 7.10 para los casos de $\mathrm{ZnO}, \mathrm{SnO}_{2}$ y $\mathrm{SnO}$. Por lo tanto, luego de analizar la convergencia de los parámetros hiperfinos, de la energía total y de la distancia entre el átomo de Cd y sus primeros vecinos oxígeno en función del parámetro RKMAX confirmamos que la convergencia está asegurada al usar un valor de 7 para dicho parámetro y 50 puntos $k$ en la IZB. Por todo esto, los cálculos APW+lo presentados en lo que resta de la presente tesis fueron realizados con estos valores.

(a)

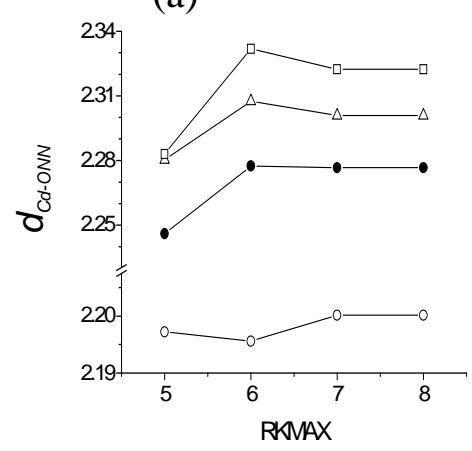

(b)

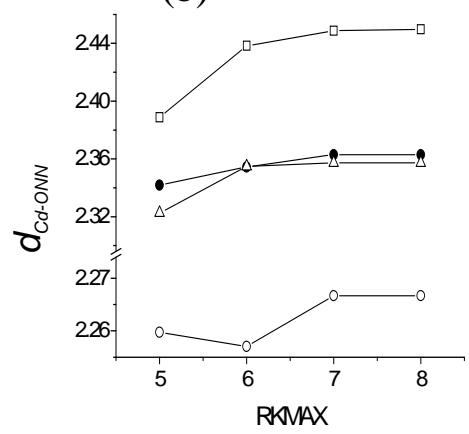

(c)

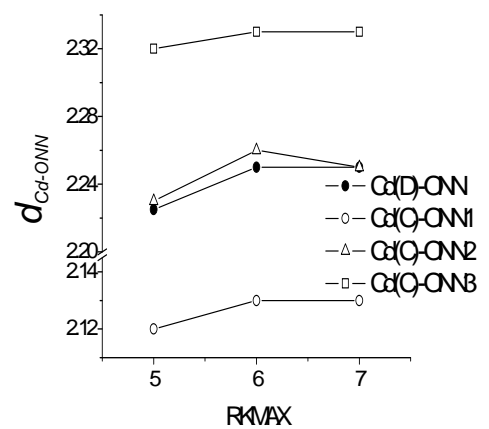

Figura 7.9: Distancia entre la impureza Cd y los oxígenos ONN vs. RKMAX para el caso de las siguientes bixbitas: (a) $\mathrm{In}_{2} \mathrm{O}_{3}$ :Cd, (b) $\mathrm{Y}_{2} \mathrm{O}_{3} \mathrm{Cd}$, (c) $\mathrm{Sc}_{2} \mathrm{O}_{3}$ : $\mathrm{Cd}$. $d_{C d-O N N}$ viene dada en $\AA$. 


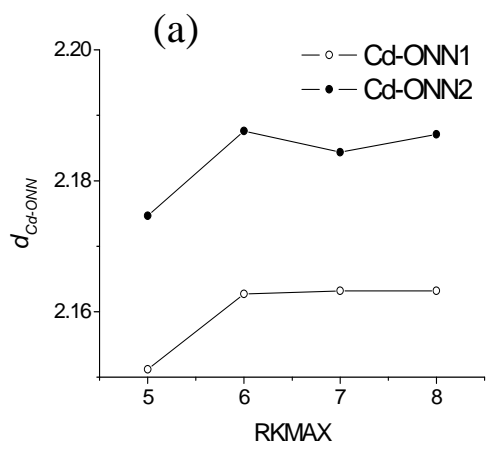

(b)

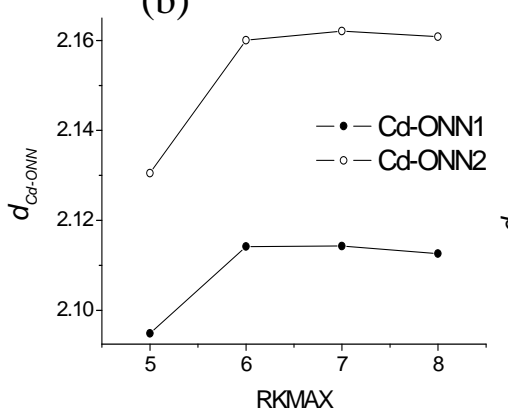

(c)

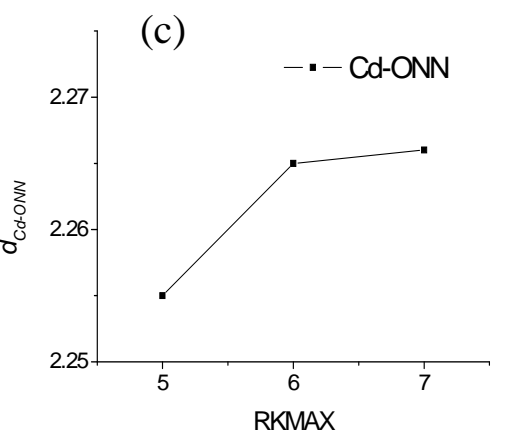

Figura 7.10: Distancia entre la impureza Cd y los oxígenos $\mathrm{O}_{\mathrm{NN}}$ vs. RKMAX para los casos: (a) $\mathrm{ZnO}: \mathrm{Cd}$, (b) $\mathrm{SnO}_{2}$ : $\mathrm{Cd}$, (c) $\mathrm{SnO}: \mathrm{Cd}$. $d_{C d-O N N}$ viene dado en $\AA$.

\section{7-2-3 Cálculos APW+lo del tensor GCE en función del estado de carga de la celda en óxidos con estructura Bixbita}

Primero estudiaremos la dependencia del GCE con el estado de carga de la celda para el caso de las bixbitas. Cabe recordar que en los sesquióxidos el catión actúa con valencia 3+ por lo que al reemplazar un catión nativo por un átomo Cd (de valencia 2+) de la red huésped, la impureza Cd actúa como simple aceptor por lo que necesita atrapar un electrón del sistema para actuar como un catión nativo. En este sentido, para el caso de las bixbitas se realizaron cálculos del GCE con celda neutra, celda descargada y con celda cargada, esto es reemplazando un catión nativo por un átomo de Cd, removiendo y agregando un electrón de la celda de cálculo, respectivamente. El caso de celda descargada (removiendo un electrón de la celda) se realizó para simular el caso de la sonda ${ }^{111} \mathrm{Cd}$ con dos huecos en su entorno. Cabe recordar que en el caso de las bixbitas la celda de cálculo está formada por una celda de 80 átomos (celda unidad de la bixbita) de los cuales 32 son cationes y 48 son oxígenos, por lo que al reemplazar un catión por una impureza Cd se obtiene una dilución de 1:32. Además, el hecho que el parámetro de red de estos óxidos sea del orden de los $10 \AA$ hace que la distancia entre impurezas sea cercana a este valor garantizando que una impureza no "vea" los efectos de las otras impurezas primeras vecinas. En primera instancia, veremos los cálculos para el caso del $\operatorname{In}_{2} \mathrm{O}_{3}: \mathrm{Cd}$. 


\section{7-2-3 (a) Cálculos del GCE en $\operatorname{In}_{2} \mathrm{O}_{3}: C d$}

Primero analicemos la densidad de estados (DOS) del sistema. En la Figura 7.11 se grafica las DOS total del sistema puro y dopado para el caso de la celda neutra y la celda cargada. En todos los casos se grafica la contribución de las esferas de los oxígenos, de las esferas de los cationes y en el caso dopado además se agrega la contribución del átomo Cd y sus primeros vecinos oxígeno. En general, todas las DOS en este trabajo se grafican con el eje de energía referido a la energía de Fermi. Desde la comparación de la DOS del $\mathrm{In}_{2} \mathrm{O}_{3}$ puro (Fig. 7.11 (e)) con las DOS de $\mathrm{In}_{2} \mathrm{O}_{3}$ :Cd con el Cd en el sitio D y en el sitio C (Fig. 7.11 (a) y (b), y Fig. 7.11 (c) y (d), respectivamente) se observa que la inclusión de la impureza en el semiconductor introduce un nivel simple aceptor en el tope de la banda de valencia del semiconductor. De hecho la integración de los estados electrónicos que están por encima del nivel de Fermi de la celda neutra (Fig. 7.11 (a)) da como resultado 1 electrón.

Como se aprecia en las DOS del caso dopado (Fig. 7.11 (a), (b), (c) y (d)), la banda de valencia corresponde mayoritariamente a los átomos oxígenos (al igual que en el caso puro), mientras que el nivel de impureza presenta contribuciones de los ONN principalmente y del átomo de Cd. De la integración de las contribuciones de las esferas atómicas a la DOS en el nivel de impureza hasta el nivel de Fermi para el caso del electrón removido y agregado a la celda neutra (Fig. 7.11 (c) y (d), respectivamente) para el Cd localizado en sitio C se deduce que un 85\% corresponde a la integración en las esferas de $\mathrm{O}$, un $10 \%$ aporta la esfera de la impureza Cd mientras que solo un $2 \%$ corresponde al In. En particular, del total de los oxígenos los $\mathrm{O}_{\mathrm{NN}}$ aportan aproximadamente un 60 \% al nivel de impureza. Por otro lado, la integración de las DOS cuando el Cd se encuentra localizado en sitio D para los casos del electrón removido y agregado a la celda neutra (Fig. 7.11 (a) y (b), respectivamente) da como resultado que la integración en las esferas de los O aportan un 75\% al nivel de impureza, el Cd aporta un 9\%, el In un 1,5 \% y finalmente la integración en las esferas de los $\mathrm{O}_{\mathrm{NN}}$ corresponde a un 50\% del nivel. De la comparación de las integraciones en ambos sistemas, se observa que existe una diferencia del 10\% de los estados electrónicos que se encuentran en las esferas de los $\mathrm{O}$ pero la contribución de los $\mathrm{O}_{\mathrm{NN}}$ es similar para los dos sistemas. Por lo tanto, en ambos casos se observa que los estados electrónicos localizados en el nivel de impureza se encuentran mayoritariamente localizados en las esferas del $\mathrm{Cd}$ y de sus $\mathrm{O}_{\mathrm{NN}}$, de hecho se obtuvo un $70 \%$ de los 
estados electrónicos del nivel de impureza para el caso de Cd en sitio C y un 60\% para el caso de Cd en sitio D.

(a)

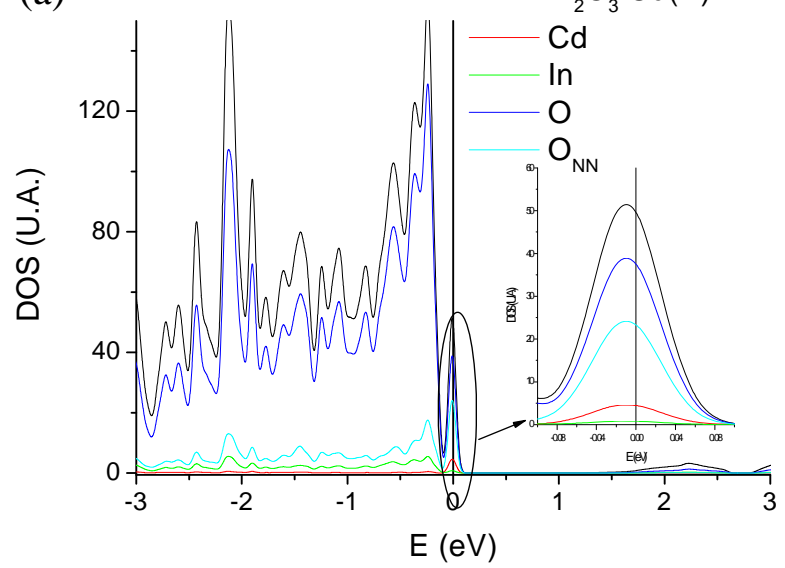

(c)

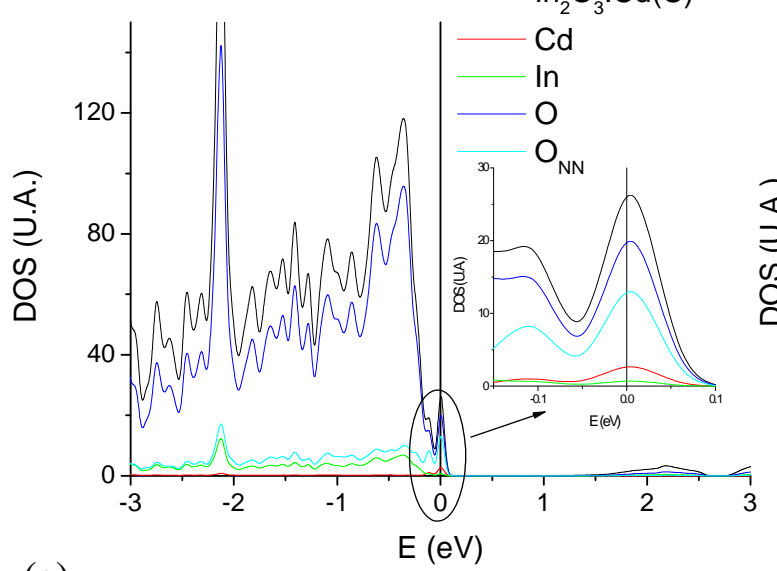

(e)

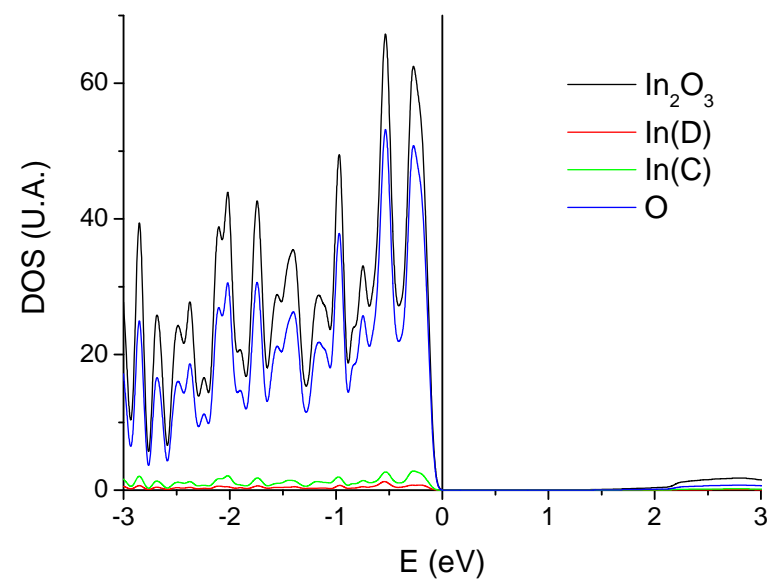

(b)

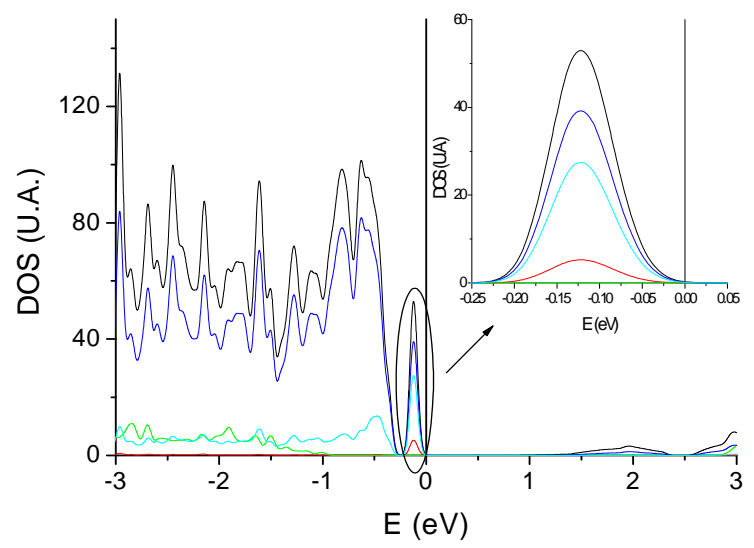

(d)

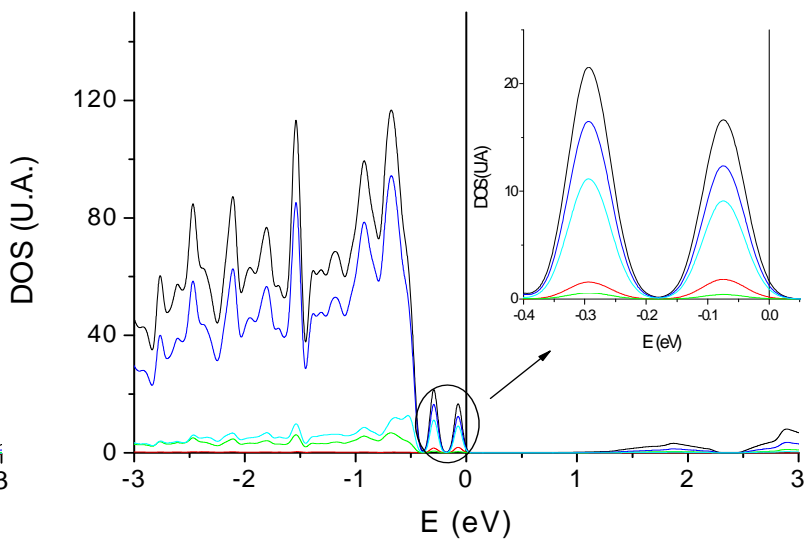

Figura 7.11: DOS del óxido $\mathrm{In}_{2} \mathrm{O}_{3}$ puro y dopado con $\mathrm{Cd}$ con las contribuciones de los átomos In y $\mathrm{O}$ de la celda, de los $\mathrm{O}_{\mathrm{NN}} \mathrm{y}$ del Cd para los casos: Cd en el sitio D (a) celda neutra, (b) celda cargada; Cd en el sitio C (c) celda neutra, (d) celda cargada; y (e) $\mathrm{In}_{2} \mathrm{O}_{3}$ puro.

En la Tabla 7.14 se muestra la dependencia del GCE con el estado de carga de la celda calculada en las aproximaciones LDA y CW-GGA. De la comparación de los resultados obtenidos en ambas aproximaciones se observa un excelente acuerdo entre 
ellas. En cuanto a la dependencia del GCE con el estado de carga, se aprecia una fuerte dependencia cuando el GCE es calculado con el Cd localizado en el sitio C. Por el contrario, los parámetros hiperfinos no presentan dependencia con el estado de carga cuando el átomo Cd está localizado en el sitio D.

Para interpretar estos resultados en la Figura 7.13 se grafican las densidades de estados parciales (PDOS) de las simetrías $d$ del Cd para celda neutra y celda cargada en el nivel de impureza.
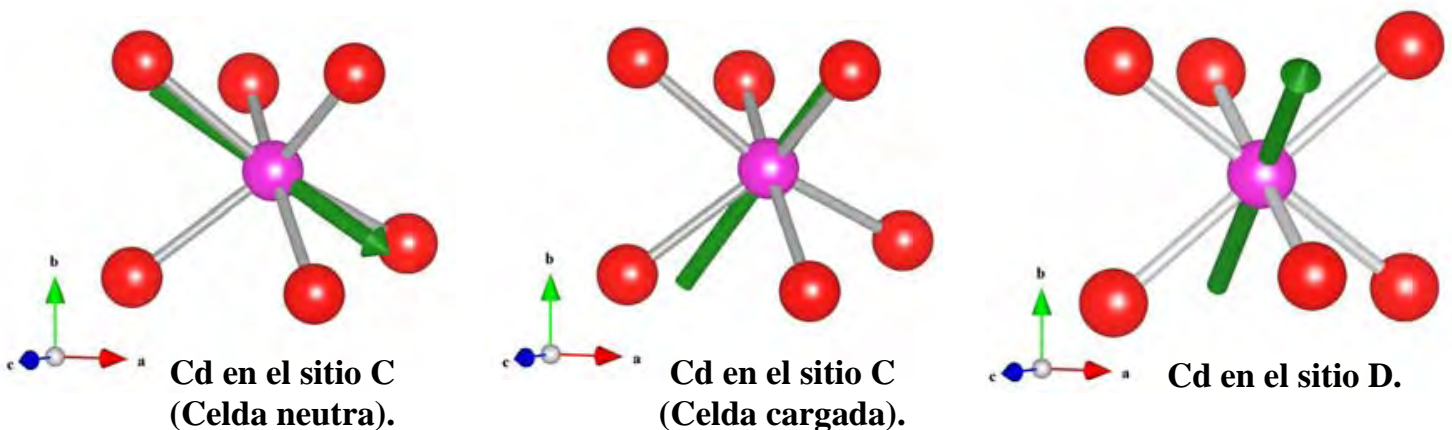

Figura 7.12: Direcciones de la componente $\mathrm{V}_{33}$ para los casos del Cd en el sitio $\mathrm{C}$ del $\mathrm{In}_{2} \mathrm{O}_{3}$ (celda neutra y cargada) y para el Cd en el sitio D.

\begin{tabular}{|c|c|c|c|c|c|c|c|c|c|}
\hline \multirow{2}{*}{$\begin{array}{c}\text { Estado de } \\
\text { carga de la } \\
\text { celda } \\
\end{array}$} & \multirow{2}{*}{ Aprox. } & \multicolumn{4}{|c|}{ Sitio D } & \multicolumn{4}{|c|}{ Sitio C } \\
\hline & & $\boldsymbol{d}_{N N}$ & $V_{33}$ & $\eta$ & Dir. $V_{33}$ & $\boldsymbol{d}_{N N}$ & $V_{33}$ & $\eta$ & Dir. $V_{33}$ \\
\hline Descargada & CW-GGA & 2.20 & +6.64 & 0.00 & {$\left[\begin{array}{lll}1 & 1 & 1\end{array}\right]$} & $\begin{array}{l}2.11 \\
2.23 \\
2.30 \\
\end{array}$ & 2.77 & 0.58 & {$\left[\begin{array}{lll}0 & 0 & 1\end{array}\right]$} \\
\hline \multirow{2}{*}{ Neutra } & LDA & 2.23 & +6.93 & 0.00 & {$\left[\begin{array}{lll}1 & 1 & 1\end{array}\right]$} & $\begin{array}{l}2.14 \\
2.25 \\
2.31\end{array}$ & -3.13 & 0.08 & {$\left[\begin{array}{lll}1 & -0.6 & 0\end{array}\right]$} \\
\hline & CW-GGA & 2.23 & +7.15 & 0.00 & {$\left[\begin{array}{lll}1 & 1 & 1\end{array}\right]$} & $\begin{array}{l}2.14 \\
2.27 \\
2.30 \\
\end{array}$ & -3.08 & 0.01 & {$\left[\begin{array}{lll}1 & -0.6 & 0\end{array}\right]$} \\
\hline \multirow{2}{*}{ Cargada } & LDA & 2.27 & +7.29 & 0.00 & {$\left[\begin{array}{lll}1 & 1 & 1\end{array}\right]$} & $\begin{array}{l}2.20 \\
2.30 \\
2.31 \\
\end{array}$ & +6.16 & 0.69 & {$\left[\begin{array}{lll}0.8 & 1 & 0\end{array}\right]$} \\
\hline & CW-GGA & 2.28 & +7.68 & 0.00 & {$\left[\begin{array}{lll}1 & 1 & 1\end{array}\right]$} & $\begin{array}{l}2.20 \\
2.30 \\
2.32\end{array}$ & +5.72 & 0.72 & {$\left[\begin{array}{lll}0.8 & 1 & 0\end{array}\right]$} \\
\hline
\end{tabular}

Tabla 7.14: Cálculos APW+lo de $d_{N N}, V_{33}$ (dirección y magnitud) y $\eta$ en función del estado de carga de la celda de $\mathrm{In}_{2} \mathrm{O}_{3}$ :Cd en las aproximaciones LDA y CW-GGA. Las distancias $d_{N N}$ están dadas en $\AA$ y $\mathrm{V}_{33}$ en unidades de $10^{21} \mathrm{~V} / \mathrm{m}^{2}$. 
Como puede apreciarse en la Figura 7.13, el tipo de simetría que aporta la integral en la esfera de $\mathrm{Cd}$ al nivel de impureza es prácticamente todo de carácter $d$. En cuanto a las simetrías $d$, para el caso de Cd en sitio C solo las simetrías $d_{x y}, d_{x z}$ y $d_{y z}$ participan en el llenado del nivel de impureza, mientras que para el caso de Cd en el sitio D, contribuyen todas las simetrías $d$ en el llenado del nivel de impureza excepto la simetría $d_{z}$. Por lo tanto, al agregar el electrón a la celda en el caso del sitio C, el GCE presenta cambios en signo, dirección y magnitud debido a que el llenado del nivel de impureza es fuertemente dependiente de las simetrías $d$, mientras que el GCE observado en el Cd localizado en el sitio D no presenta modificaciones al agregar un electrón al sistema debido a que el cambio en las simetrías $d$ se realiza de manera uniforme.
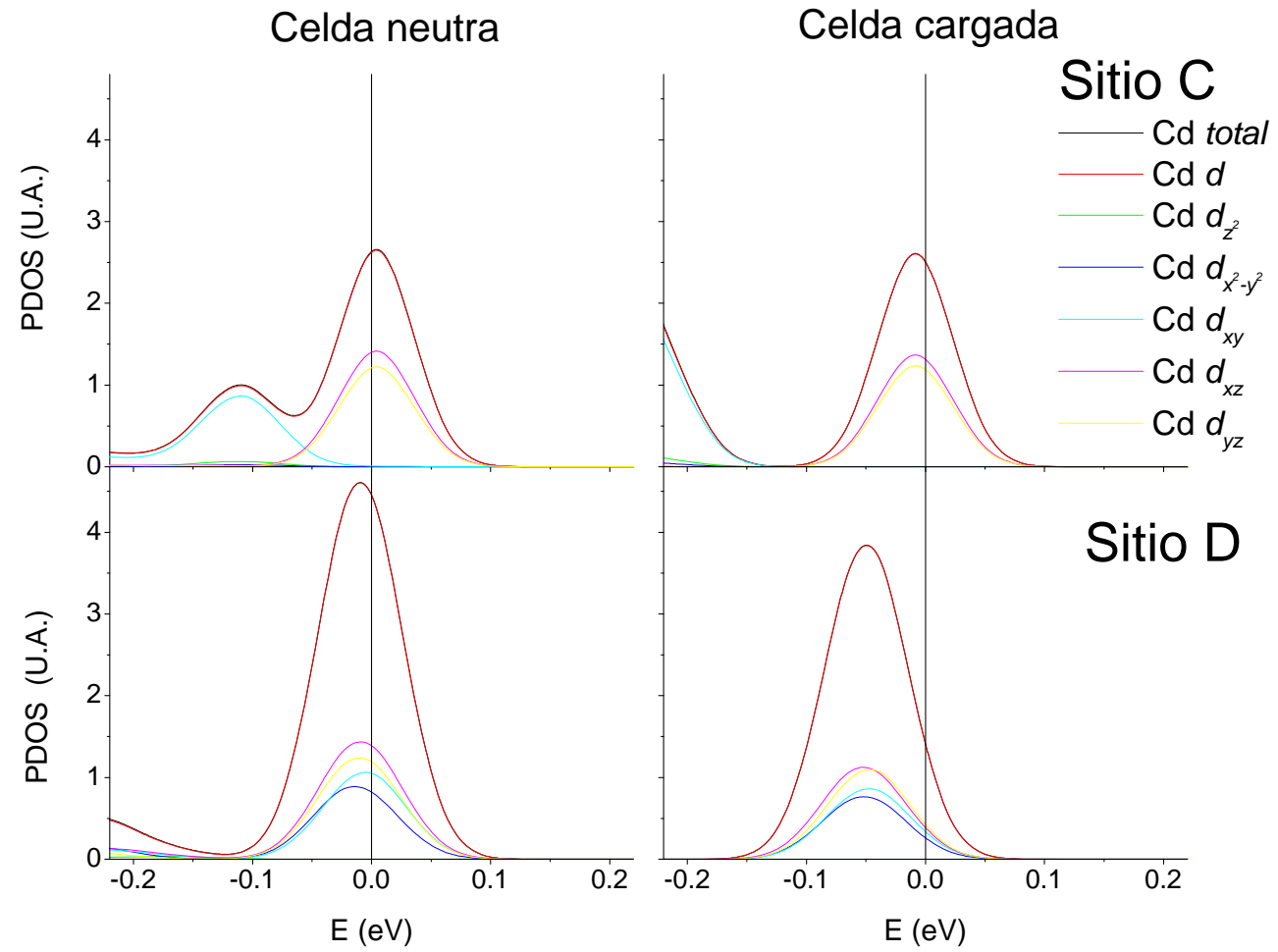

Figura 7.13: PDOS de las simetrías $d$ del $\mathrm{Cd}$ en $\mathrm{In}_{2} \mathrm{O}_{3}$ :Cd. Las PDOS de la izquierda corresponden a celda neutra mientras que en la derecha se grafican las de la celda cargada. Los gráficos superiores corresponden a Cd en sitio C y las PDOS inferiores a Cd en sitio D.

Por último, en la Tabla 7.15 se detallan las contribuciones $p, d$ y s- $d$ al GCE dentro de la esfera de Cd al GCE en función del estado de carga de la celda calculado en el 
sitio C mientras que en la Tabla 7.16 se muestran las mismas contribuciones para el caso de Cd en sitio D.

Como puede apreciarse en la Tabla 7.15, las contribuciones $p$ y $d$ de las tres componentes $V_{i i}$ del tensor presentan cambios de signo y magnitud al agregar un electrón a la celda. Mientras que tales contribuciones para el caso de Cd en el sitio D (Tabla 7.16) se ven modificadas en menos del $10 \%$ del valor de cada componente $V_{i i}$ al cambiar el estado de carga de la celda con lo que el GCE del sitio D relativamente es independiente del estado de carga de la celda. Por último se observa en todos los casos que las contribuciones $p$ están por encima de las $d$, mientras que el aporte de la simetría $s-d$ al GCE es despreciable.

\section{Cd en sitio C}

\begin{tabular}{cccccccccc}
\hline & \multicolumn{3}{c}{ Celda descargada } & \multicolumn{3}{c}{ Celda neutra } & \multicolumn{3}{c}{ Celda cargada } \\
\hline & $\boldsymbol{V}_{\mathbf{1 1}}$ & $\boldsymbol{V}_{\mathbf{2 2}}$ & $\boldsymbol{V}_{\mathbf{3 3}}$ & $\boldsymbol{V}_{\mathbf{1 1}}$ & $\boldsymbol{V}_{\mathbf{2 2}}$ & $\boldsymbol{V}_{\mathbf{3 3}}$ & $\boldsymbol{V}_{\mathbf{1 1}}$ & $\boldsymbol{V}_{\mathbf{2 2}}$ & $\boldsymbol{V}_{\mathbf{3 3}}$ \\
\hline $\boldsymbol{p}$ & +8.79 & -6.09 & -2.70 & -2.02 & +6.95 & -4.92 & -0.84 & -3.37 & +4.21 \\
\hline $\boldsymbol{d}$ & -9.56 & +4.11 & +5.45 & +3.56 & -5.51 & +1.94 & +0.04 & -1.66 & +1.62 \\
\hline $\boldsymbol{s}-\boldsymbol{d}$ & +0.16 & -0.19 & +0.03 & +0.02 & +0.14 & -0.17 & +0.01 & +0.12 & -0.13 \\
\hline total & -0.58 & -2.19 & 2.77 & +1.50 & +1.56 & -3.06 & -0.77 & -4.91 & +5.70 \\
\hline \hline
\end{tabular}

Tabla 7.15: Cálculos APW+lo de las contribuciones $p, d$ y $s-d$ al tensor GCE en función del estado de carga de la celda (aproximación CW-GGA) para Cd en sitio C en $\operatorname{In}_{2} \mathrm{O}_{3} . V_{i i}(i=1,2,3)$ viene dado en unidades de $10^{21} \mathrm{~V} / \mathrm{m}^{2}$.

\section{Cd en Sitio D}

Celda neutra

Celda cargada

\begin{tabular}{ccccccc}
\hline & $\boldsymbol{V}_{\mathbf{1 1}}$ & $\boldsymbol{V}_{\mathbf{2 2}}$ & $\boldsymbol{V}_{\mathbf{3 3}}$ & $\boldsymbol{V}_{\mathbf{1 1}}$ & $\boldsymbol{V}_{\mathbf{2 2}}$ & $\boldsymbol{V}_{\mathbf{3 3}}$ \\
\hline $\boldsymbol{p}$ & -3.14 & -3.14 & +6.28 & -2.98 & -2.98 & +5.95 \\
\hline $\boldsymbol{d}$ & -0.50 & -0.50 & +1.00 & -0.93 & -0.93 & +1.86 \\
\hline $\boldsymbol{s}-\boldsymbol{d}$ & +0.08 & +0.08 & -0.17 & +0.09 & +0.09 & -0.17 \\
\hline total & -3.55 & -3.55 & 7.11 & -3.82 & -3.82 & 7.64 \\
\hline \hline
\end{tabular}

Tabla 7.16: Cálculos APW+lo de las contribuciones $p, d$ y $s-d$ al tensor GCE en función del estado de carga de la celda (aproximación CW-GGA) para Cd en sitio D en $\operatorname{In}_{2} \mathrm{O}_{3} . V_{i i}(i=1,2,3)$ viene dado en unidades de $10^{21} \mathrm{~V} / \mathrm{m}^{2}$. 


\section{7-2-3 (b) Cálculos del GCE en $\mathrm{Y}_{2} \mathrm{O}_{3}: C d$}

En la Figura 7.14 se grafican las DOS del $\mathrm{Y}_{2} \mathrm{O}_{3}$ puro y del $\mathrm{Y}_{2} \mathrm{O}_{3}$ :Cd, las contribuciones de los oxígenos, de los átomos Y en los dos casos, y adicionalmente, la contribución de la impureza Cd y sus primeros vecinos oxígeno para el caso. Desde la comparación de las DOS correspondientes a $\mathrm{Y}_{2} \mathrm{O}_{3}$ puro (Fig. 7.14 (e)) y $\mathrm{Y}_{2} \mathrm{O}_{3}$ :Cd (Fig. 7.14 (a), (b), (c) y (d)) se observa que la impureza Cd introduce un nivel simple aceptor en el gap del semiconductor cercano a la banda de valencia tal como se observó en la bixbita $\mathrm{In}_{2} \mathrm{O}_{3}$. En particular, la integración de los estados electrónicos que están por encima del nivel de Fermi de la celda neutra (Fig. 7.14 (a)) da como resultado 1 electrón.

Desde los gráficos de las DOS se observa que la mayor contribución a la banda de valencia corresponde a las esferas de los oxígenos. En cuanto a las contribuciones de las esferas atómicas integrando los estados electrónicos del nivel de impureza hasta el nivel de Fermi para el caso del electrón removido y agregado a la celda neutra del Cd en el sitio D (Fig. 7.14 (a) y (b), respectivamente), se observa que las esferas de los oxígenos aportan un 75\%, la integración en la esfera del Cd un 8\%, mientras que las esferas de In aportan $2 \%$ y los $\mathrm{O}_{\mathrm{NN}}$ contribuyen con un $50 \%$ al nivel de impureza. En el caso del electrón removido y agregado a la celda neutra para el Cd en el sitio C (Fig. 7.14 (c) y (d), respectivamente), los resultados son similares: los oxígenos contribuyen con 77\%, la esfera del Cd con 6\%, las esferas de $\mathrm{Y}$ con $2 \%$ y los $\mathrm{O}_{\mathrm{NN}}$ aportan un $45 \%$. Finalmente, se tiene que un 60\% (50\%) de los estados electrónicos del nivel de impureza se encuentran en las esferas de los $\mathrm{O}_{\mathrm{NN}}$ y del Cd para el caso de Cd en sitio D (C). Por lo tanto, de la contribución de las esferas atómicas a los estados electrónicos correspondientes al electrón removido y agregado a la celda neutra del $\mathrm{Y}_{2} \mathrm{O}_{3}$ :Cd tanto para el Cd en el sitio D como en el sitio C, se deduce que tales estados electrónicos se localizan esencialmente en el entorno del Cd y sus primeros vecinos oxígeno. 
(a)
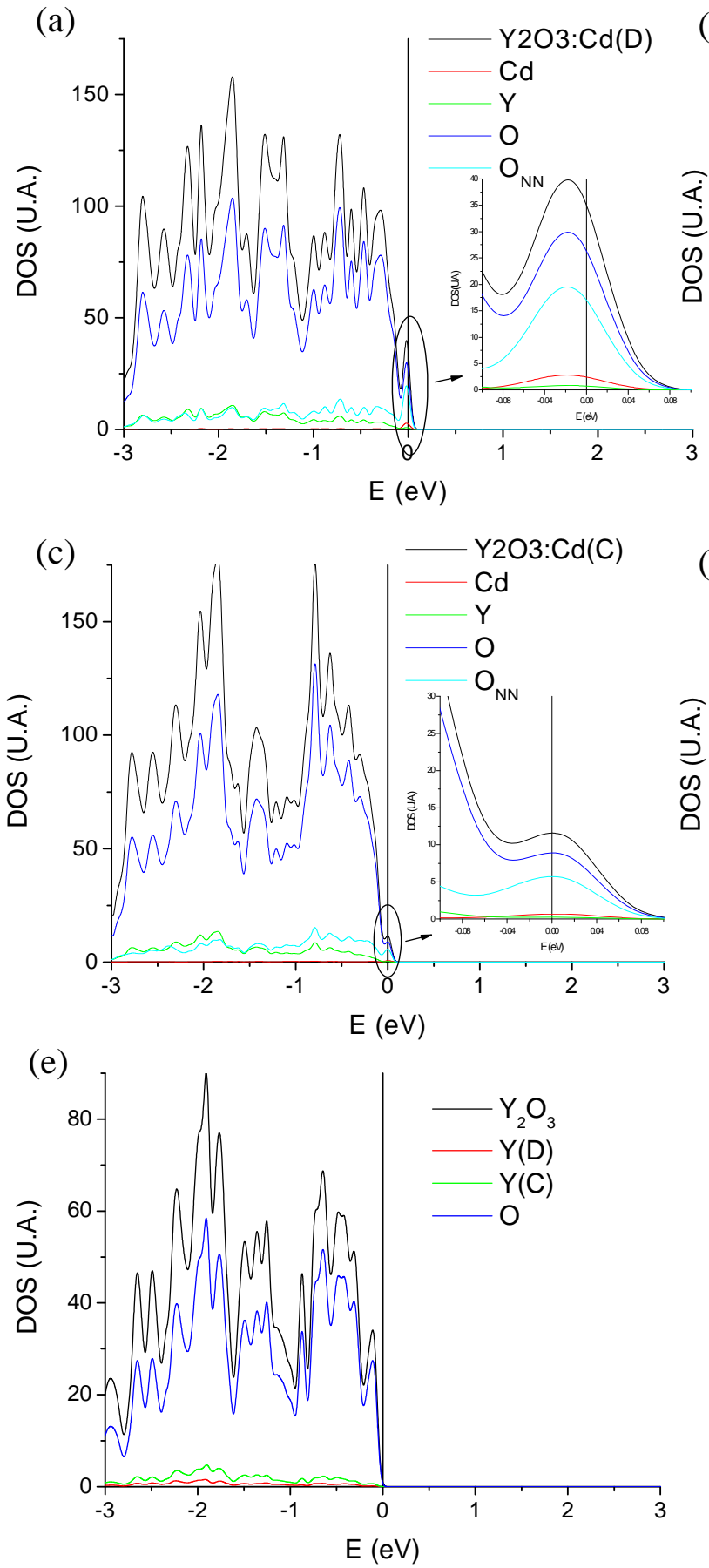

(b)
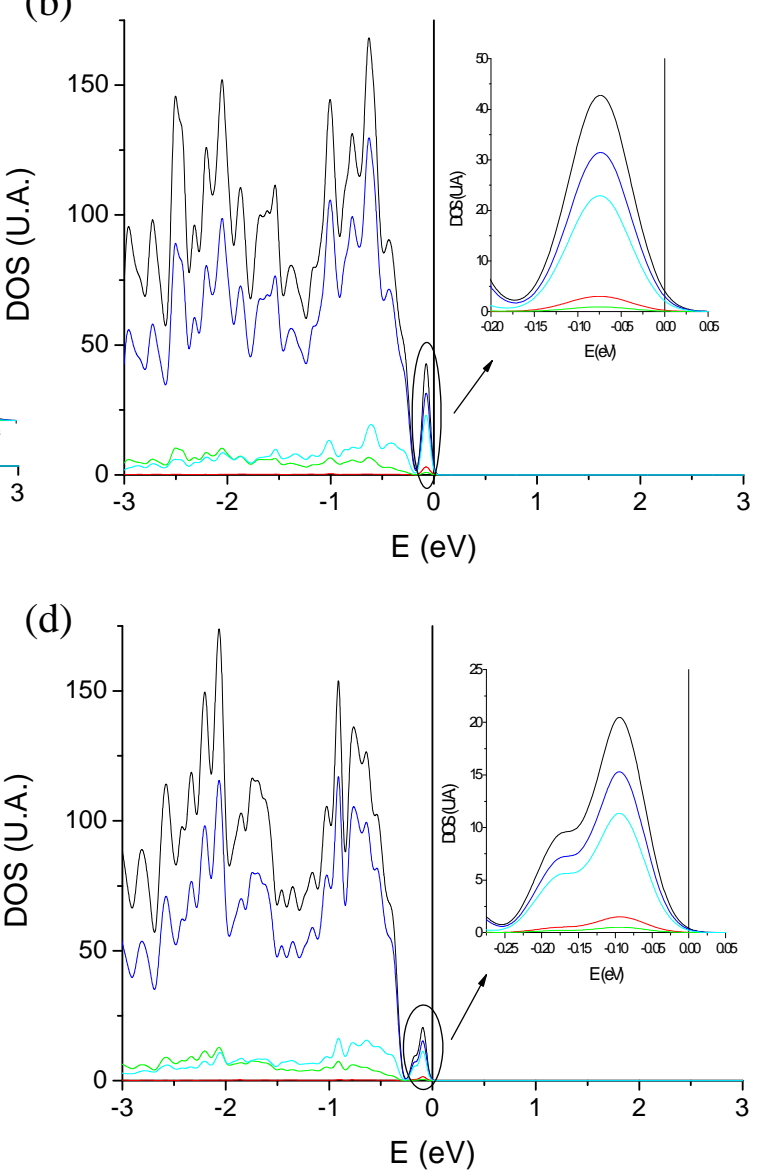

Figura 7.14: DOS del óxido $\mathrm{Y}_{2} \mathrm{O}_{3}$ puro y dopado con $\mathrm{Cd}$, y las contribuciones de los átomos $\mathrm{Y}$ y $\mathrm{O}$ de la celda y de los $\mathrm{O}_{\mathrm{NN}}$ para los casos: Cd localizado en el sitio D (a) celda neutra, (b) celda cargada; Cd en el sitio C (c) celda neutra, (d) celda cargada; y (e) DOS del $\mathrm{Y}_{2} \mathrm{O}_{3}$ puro.

En la Tabla 7.17 se detalla la dependencia del tensor GCE con el estado de carga de la celda calculada con el método APW+lo en la aproximaciones LDA y CW-GGA. Al igual que en el caso del $\mathrm{In}_{2} \mathrm{O}_{3}: \mathrm{Cd}$, se observa un excelente acuerdo entre las predicciones obtenidas con LDA y con CW-GGA. Además, se observa que el GCE no presenta cambios con el estado de carga para el caso de Cd localizado en sitio D, mientras que cambia en magnitud, signo y dirección cuando se agrega un electrón a la celda neutra para Cd localizado en el sitio C. Este resultado también es similar al caso 
del $\mathrm{In}_{2} \mathrm{O}_{3}$ :Cd y análogamente se atribuye al tipo de simetría de cada sitio: el sitio D es axialmente simétrico mientras que el sitio C presenta una alta asimetría.
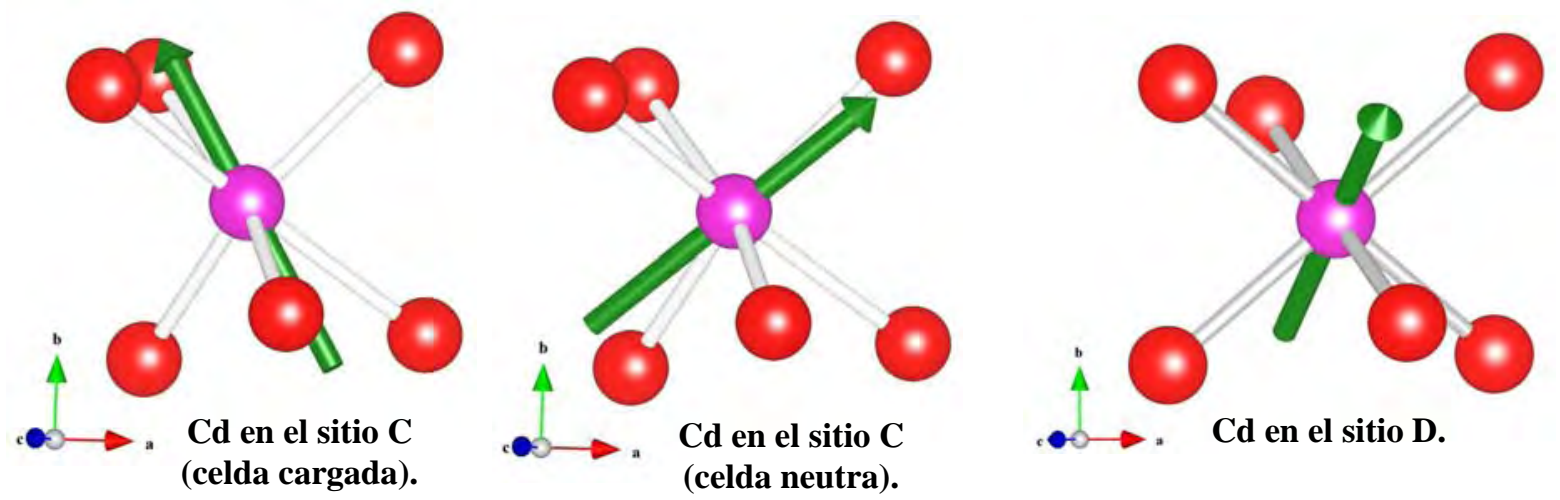

Figura 7.15: Direcciones de la componente $\mathrm{V}_{33}$ para los casos del Cd en el sitio C del $\mathrm{Y}_{2} \mathrm{O}_{3}$ (celda neutra y cargada) y para el Cd en el sitio D.

\begin{tabular}{|c|c|c|c|c|c|c|c|c|c|}
\hline \multirow{2}{*}{$\begin{array}{l}\text { Estado de } \\
\text { carga de la } \\
\text { celda }\end{array}$} & \multirow{2}{*}{ Aprox. } & \multicolumn{4}{|c|}{ Sitio D } & \multicolumn{4}{|c|}{ Sitio C } \\
\hline & & $\boldsymbol{d}_{N N}$ & $V_{33}$ & $\eta$ & Dir. $V_{33}$ & $\boldsymbol{d}_{N N}$ & $V_{33}$ & $\eta$ & Dir. $V_{33}$ \\
\hline Descargada & CW-GGA & 2.28 & +7.15 & 0.00 & {$\left[\begin{array}{lll}1 & 1 & 1\end{array}\right]$} & $\begin{array}{l}2.19 \\
2.33 \\
2.35\end{array}$ & -2.05 & 0.54 & {$\left[\begin{array}{lll}-0.4 & 1 & 0\end{array}\right]$} \\
\hline \multirow{2}{*}{ Neutra } & LDA & 2.31 & +7.10 & 0.00 & {$\left[\begin{array}{lll}1 & 1 & 1\end{array}\right]$} & $\begin{array}{l}2.20 \\
2.36 \\
2.37\end{array}$ & -3.00 & 0.27 & {$\left[\begin{array}{lll}-0.6 & 1 & 0\end{array}\right]$} \\
\hline & CW-GGA & 2.32 & +7.25 & 0.00 & {$\left[\begin{array}{lll}1 & 1 & 1\end{array}\right]$} & $\begin{array}{l}2.22 \\
2.36 \\
2.38\end{array}$ & -2.58 & 0.05 & {$\left[\begin{array}{lll}-0.5 & 1 & 0\end{array}\right]$} \\
\hline \multirow{2}{*}{ Cargada } & LDA & 2.34 & +7.21 & 0.00 & {$\left[\begin{array}{lll}1 & 1 & 1\end{array}\right]$} & $\begin{array}{l}2.26 \\
2.36 \\
2.42\end{array}$ & +4.06 & 0.86 & {$\left[\begin{array}{lll}1 & 0.8 & 0\end{array}\right]$} \\
\hline & CW-GGA & 2.36 & +7.28 & 0.00 & {$\left[\begin{array}{lll}1 & 1 & 1\end{array}\right]$} & $\begin{array}{l}2.27 \\
2.35 \\
2.45\end{array}$ & +3.73 & 0.88 & {$\left[\begin{array}{lll}1 & 0.7 & 0\end{array}\right]$} \\
\hline
\end{tabular}

Tabla 7.17: Cálculos APW+lo de $d_{N N}, V_{33}$ (dirección y magnitud) y $\eta$ en función del estado de carga de la celda de $\mathrm{Y}_{2} \mathrm{O}_{3}$ :Cd en las aproximaciones LDA y CW-GGA. Las distancias $d_{N N}$ están dadas en $\AA$ y $\mathrm{V}_{33}$ en unidades de $10^{21} \mathrm{~V} / \mathrm{m}^{2}$.

En la Figura 7.16 se grafican las simetrías $d$ del Cd para los casos de celda cargada y celda neutra y para Cd en sitio $\mathrm{C}$ y sitio D de la estructura del $\mathrm{Y}_{2} \mathrm{O}_{3}$. Al igual que en el $\mathrm{In}_{2} \mathrm{O}_{3}: \mathrm{Cd}$, en el llenado del nivel de impureza cuando el $\mathrm{Cd}$ se localiza en sitio $\mathrm{C}$ solo contribuyen (de manera diferente) las simetrías $d_{x y}, d_{x z}$ y $d_{y z}$ mientras que en el caso de 
Cd en sitio D contribuyen todas las simetrías uniformemente excepto la simetría $d_{z^{2}}$. Por lo que el GCE calculado en la impureza Cd en sitio $C$ deviene en un observable fuertemente dependiente del estado de carga mientras que dicho observable no presenta dependencia cuando el Cd se encuentra en sitio D.

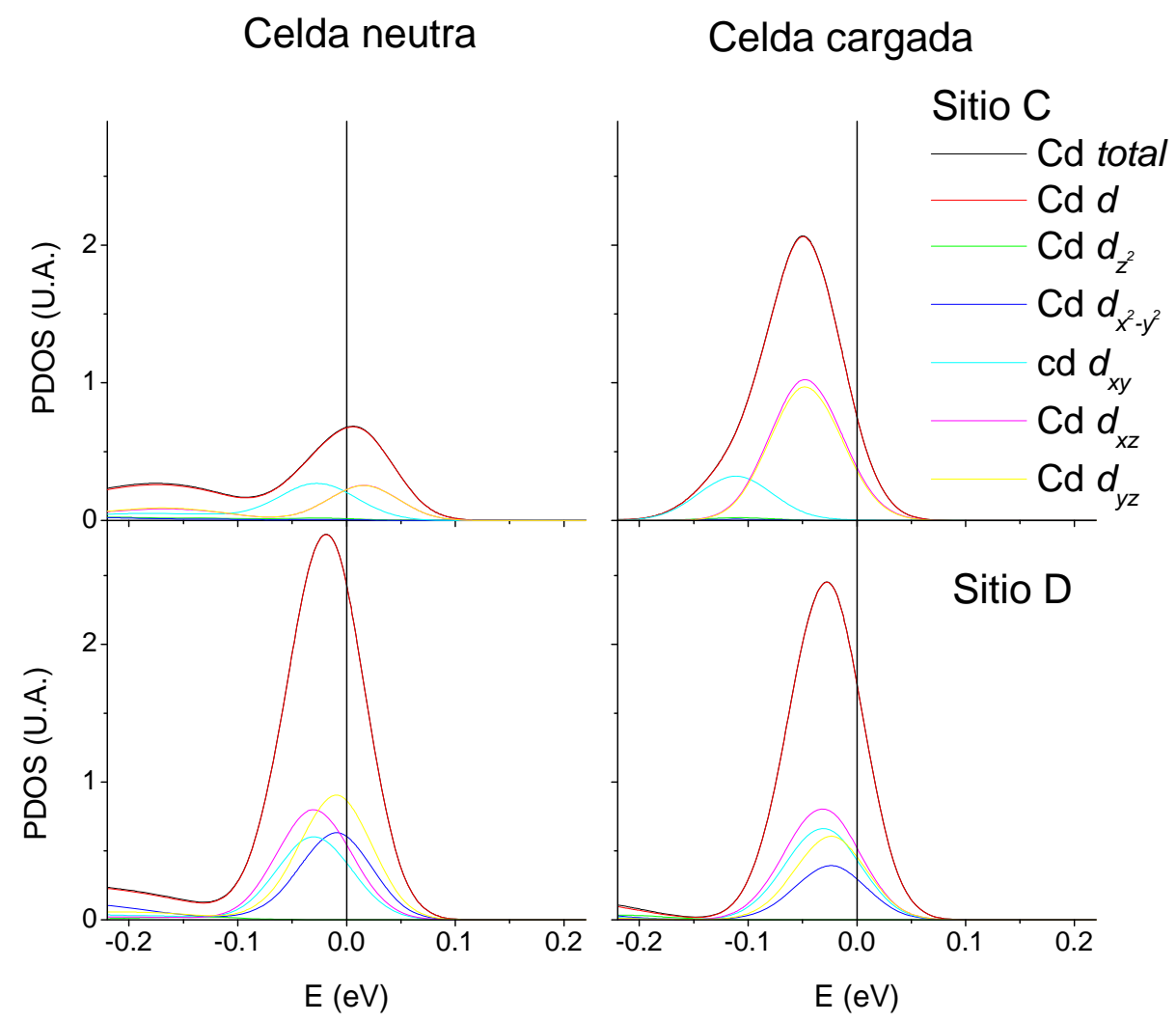

Figura 7.16: PDOS de las simetrías $d$ del $\mathrm{Cd}$ en $\mathrm{Y}_{2} \mathrm{O}_{3}$. Las PDOS de la izquierda corresponden a celda neutra mientras que en la derecha se grafican las de la celda cargada. Los gráficos superiores corresponden a $\mathrm{Cd}$ en sitio $\mathrm{C}$ y las PDOS inferiores a Cd en sitio D.

Para concluir con el estudio del GCE en el sistema $\mathrm{Y}_{2} \mathrm{O}_{3}: \mathrm{Cd}$, se detallan las contribuciones al GCE de carácter $p, d$ y $s$ - $d$ dentro de la esfera del Cd. Los resultados de la Tabla 7.18 corresponden a Cd en sitio C y en la Tabla 7.19 se describen las contribuciones para el caso de $\mathrm{Cd}$ en sitio $\mathrm{D}$. Al igual que en el caso de $\operatorname{In}_{2} \mathrm{O}_{3}$ :Cd, se observa que las contribuciones $p$ son mayores que las contribuciones $d$. Además las contribuciones $s-d$ son despreciables. Finalmente, se observa que para Cd en sitio C las componentes $V_{i i}$ presentan una fuerte dependencia con el estado de carga tanto en la contribución $p$ como en la $d$, mientras que la variación con el estado de carga del GCE calculado en sitio D se vuelve despreciable en ambas simetrías. 


\section{Cd en sitio C}

\begin{tabular}{cccccccccc}
\hline & \multicolumn{2}{c}{ Celda descargada } & \multicolumn{3}{c}{ Celda neutra } & \multicolumn{3}{c}{ Celda cargada } \\
\hline & $\boldsymbol{V}_{\mathbf{1 1}}$ & $\boldsymbol{V}_{\mathbf{2 2}}$ & $\boldsymbol{V}_{\mathbf{3 3}}$ & $\boldsymbol{V}_{\mathbf{1 1}}$ & $\boldsymbol{V}_{\mathbf{2 2}}$ & $\boldsymbol{V}_{\mathbf{3 3}}$ & $\boldsymbol{V}_{\mathbf{1 1}}$ & $\boldsymbol{V}_{\mathbf{2 2}}$ & $\boldsymbol{V}_{\mathbf{3 3}}$ \\
\hline $\boldsymbol{p}$ & +5.55 & -1.63 & -3.92 & -1.32 & +4.49 & -3.17 & -0.57 & -2.09 & +2.67 \\
\hline $\boldsymbol{d}$ & -5.28 & +3.19 & +2.08 & +2.51 & -3.30 & +0.79 & +0.34 & -1.51 & +1.17 \\
\hline $\boldsymbol{s}-\boldsymbol{d}$ & +0.11 & +0.02 & -0.14 & +0.02 & +0.10 & -0.12 & +0.01 & +0.08 & -0.09 \\
\hline total & +0.46 & +1.58 & -2.04 & +1.20 & +1.37 & -2.57 & -0.22 & -3.50 & +3.72 \\
\hline \hline
\end{tabular}

Tabla 7.18: Cálculos APW+lo de las contribuciones $p, d$ y $s-d$ al tensor GCE en función del estado de carga de la celda (aproximación CW-GGA) para Cd en sitio C en $\mathrm{Y}_{2} \mathrm{O}_{3} . V_{i i}(i=1,2,3)$ viene dado en unidades de $10^{21} \mathrm{~V} / \mathrm{m}^{2}$.

\section{Cd en Sitio D}

\section{Celda neutra}

Celda cargada

\begin{tabular}{ccccccc}
\hline & $\boldsymbol{V}_{\mathbf{1 1}}$ & $\boldsymbol{V}_{\mathbf{2 2}}$ & $\boldsymbol{V}_{\mathbf{3 3}}$ & $\boldsymbol{V}_{\mathbf{1 1}}$ & $\boldsymbol{V}_{\mathbf{2 2}}$ & $\boldsymbol{V}_{\mathbf{3 3}}$ \\
\hline $\boldsymbol{p}$ & -2.84 & -2.84 & +5.68 & -2.66 & -2.66 & +5.33 \\
\hline $\boldsymbol{d}$ & -0.85 & -0.85 & +1.69 & -1.04 & -1.04 & +2.08 \\
\hline $\boldsymbol{s}-\boldsymbol{d}$ & +0.09 & +0.09 & -0.18 & +0.09 & +0.09 & -0.18 \\
\hline total & -3.59 & -3.59 & +7.18 & -3.61 & -3.61 & +7.22 \\
\hline \hline
\end{tabular}

Tabla 7.19: Cálculos APW+lo de las contribuciones $p, d$ y $s-d$ al tensor GCE en función del estado de carga de la celda (aproximación CW-GGA) para Cd en sitio D en $\mathrm{Y}_{2} \mathrm{O}_{3} . V_{i i}(i=1,2,3)$ viene dado en unidades de $10^{21} \mathrm{~V} / \mathrm{m}^{2}$.

\section{7-2-3 (c) Cálculos del GCE en $\mathrm{Sc}_{2} \mathrm{O}_{3}: \mathrm{Cd}$}

En la Figura 7.17 se grafican las DOS correspondientes a la bixbita $\mathrm{Sc}_{2} \mathrm{O}_{3}$ pura y dopada con Cd. Además, se grafican las contribuciones de las esferas de los átomos O y de los cationes Sc para los casos puro y dopado, y adicionalmente, las contribuciones de los primeros vecinos $\mathrm{O}_{\mathrm{NN}}$ y de la impureza $\mathrm{Cd}$ en el caso dopado. Desde la comparación de las DOS correspondientes a $\mathrm{Sc}_{2} \mathrm{O}_{3}$ puro (Fig. 7.17 (e)) y a $\mathrm{Sc}_{2} \mathrm{O}_{3}$ dopado (Fig. 7.17 (a), (b), (c) y (d)) se observa que la inclusión de la impureza Cd en la red huésped introduce un nivel simple aceptor en el tope de la banda de valencia tal como se vio en los otros dos sesquióxidos abordados en esta Tesis. 
Como en el resto de las bixbitas, se puede observar que en todas las DOS graficadas en la Fig. 7.17 la banda de valencia presenta esencialmente la contribución de los átomos O. En cuanto a la integración de las DOS en el nivel de impureza, para el caso del electrón removido y agregado a la celda neutra para el Cd en el sitio D (Fig. 7.17 (a) y (b), respectivamente) se obtuvo que los $\mathrm{O}$ aportan un 76\% de los estados, los $\mathrm{O}_{\mathrm{NN}}$ un 42\%, el Cd un 7\% y los átomos de Sc un 3\%. Para el caso del electrón removido y agregado a la celda neutra para el Cd en el sitio C (Fig. 7.17 (c) y (d), respectivamente) se tiene que los $\mathrm{O}$ contribuyen con un 67\%, los vecinos $\mathrm{O}_{\mathrm{NN}}$ un $44 \%$, el $\mathrm{Cd}$ un $9 \%$ y los Sc un 4\%. Por lo tanto, de la integración se deduce que la esfera del Cd junto con las esferas de su entorno $\left(\mathrm{O}_{\mathrm{NN}}\right)$ abarcan aproximadamente un $50 \%$ de los estados electrónicos del nivel de impureza, un resultado similar se obtuvo para $\mathrm{Y}_{2} \mathrm{O}_{3}$ : $\mathrm{Cd}$ mientras que para $\mathrm{In}_{2} \mathrm{O}_{3}$ :Cd la contribución del Cd y los $\mathrm{O}_{\mathrm{NN}}$ es del 60\% (70\%) para Cd en sitio C (D). Entonces, la localización del electrón removido y agregado a la celda neutra tanto para el caso del Cd en el sitio C corresponde al entorno de la impureza Cd y sus primeros vecinos oxígeno.

En la Tabla 7.20 se detalla la dependencia del GCE (en el sitio del Cd) con el estado de carga de la celda con las aproximaciones LDA, CW-GGA y GGA. Además, con la aproximación CW-GGA se estudiaron tres estados de carga: reemplazando un átomo de Sc por uno de Cd (celda neutra), agregando un electrón a la celda (celda cargada) simulando el caso del entorno del Cd sin huecos electrónicos y removiendo un electrón de la celda (celda descargada) con el propósito de simular el entorno del Cd con dos huecos electrónicos en su entorno. 
(a)

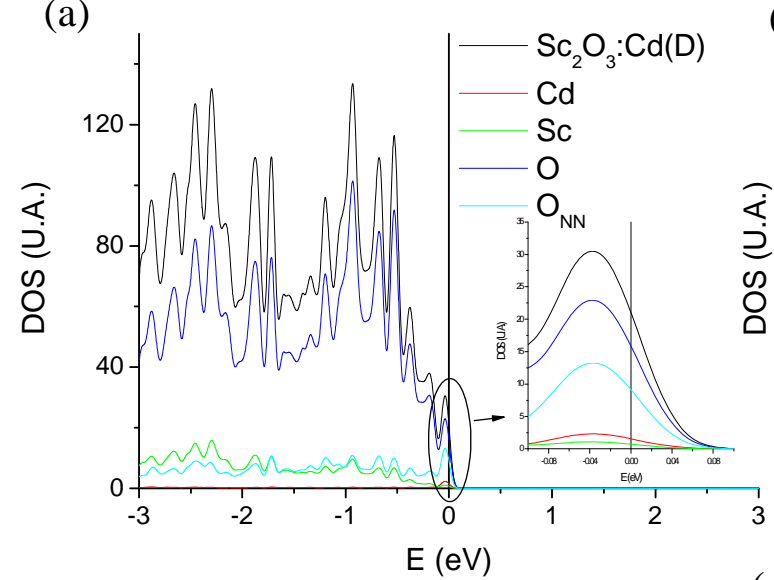

(c)

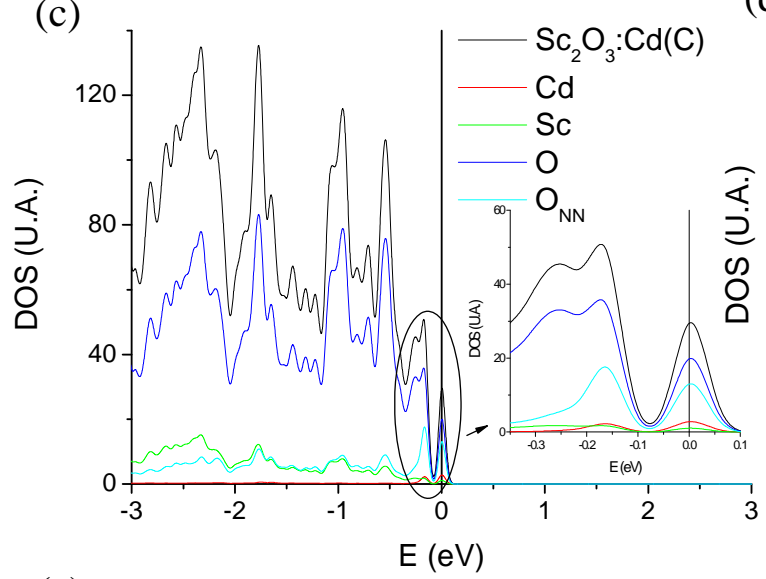

(e)

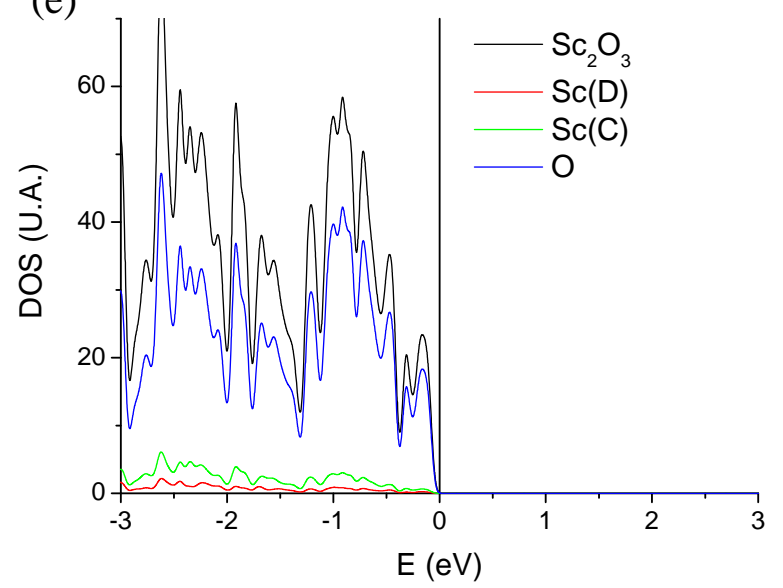

(b)

(d)

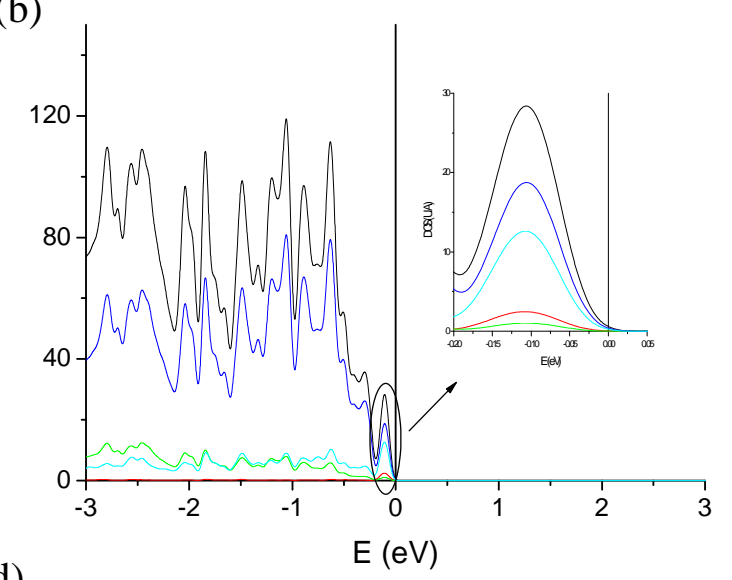

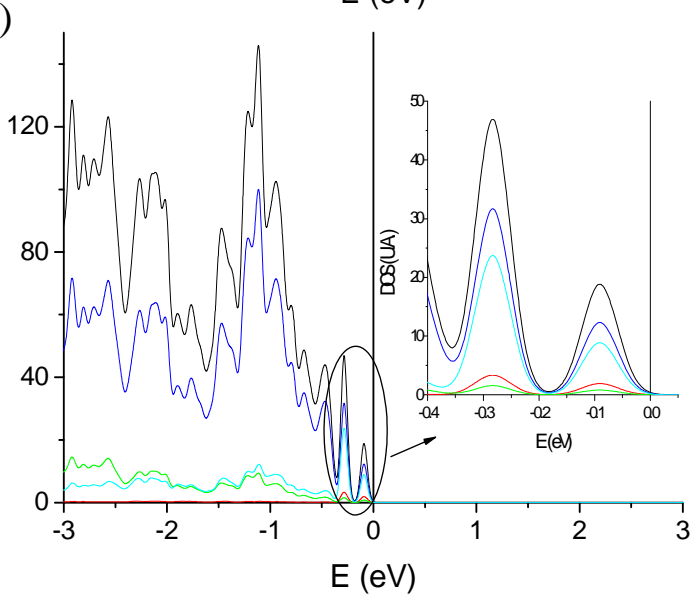

Figura 7.17: DOS del óxido $\mathrm{Sc}_{2} \mathrm{O}_{3}$ :Cd, y de las contribuciones de los átomos Sc y O de la celda y de los $\mathrm{O}_{\mathrm{NN}}$ para los casos: $\mathrm{Cd}$ en el sitio D (a) celda neutra, (b) celda cargada; Cd en el sitio C (c) celda neutra; (d) celda cargada; y (e) DOS de $\mathrm{Sc}_{2} \mathrm{O}_{3}$ puro.

Como se puede deducir de la Tabla 7.20, se observa un excelente acuerdo entre las tres aproximaciones utilizadas en esta bixbita. Por otro lado, el GCE observado en el sitio D del Cd no presenta dependencia con el estado de carga de la celda, mientras que para el caso de Cd en sitio C se aprecia una muy fuerte dependencia puesto que al remover o agregar un electrón al sistema $V_{33}$ cambia en magnitud y signo, el parámetro de asimetría duplica su valor y la dirección de $V_{33}$ también se ve alterada en especial al remover carga negativa de la celda neutra [Muñoz 2009]. 


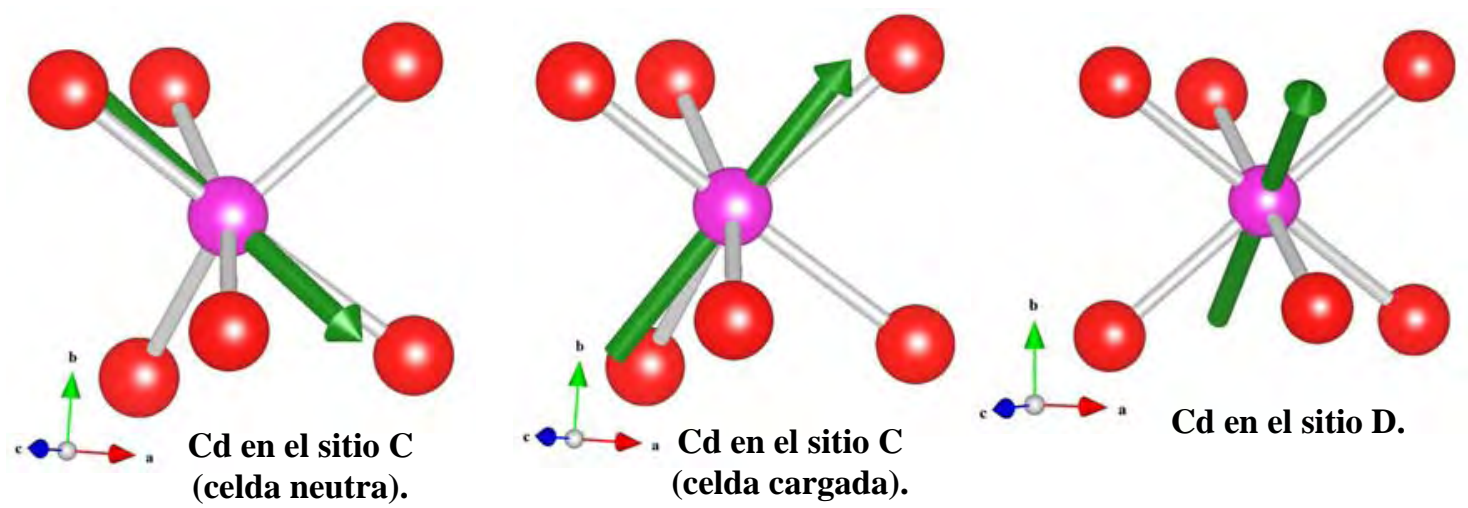

Figura 7.18: Direcciones de la componente $\mathrm{V}_{33}$ para los casos del $\mathrm{Cd}$ en el sitio $\mathrm{C}$ del $\mathrm{Sc}_{2} \mathrm{O}_{3}$ (celda neutra y cargada) y para el Cd en el sitio D.

\begin{tabular}{|c|c|c|c|c|c|c|c|c|c|}
\hline \multirow{2}{*}{$\begin{array}{c}\text { Estado de } \\
\text { carga de la } \\
\text { celda }\end{array}$} & \multirow{2}{*}{ Aprox. } & \multicolumn{4}{|c|}{ Sitio D } & \multicolumn{4}{|c|}{ Sitio C } \\
\hline & & $\boldsymbol{d}_{N N}$ & $V_{33}$ & $\eta$ & Dir. $V_{33}$ & $\boldsymbol{d}_{N N}$ & $V_{33}$ & $\eta$ & Dir. $V_{33}$ \\
\hline Descargada & $\begin{array}{l}\text { CW- } \\
\text { GGA }\end{array}$ & 2.22 & +8.24 & 0.00 & {$\left[\begin{array}{lll}1 & 1 & 1\end{array}\right]$} & $\begin{array}{l}2.10 \\
2.24 \\
2.32\end{array}$ & +3.06 & 0.64 & {$\left[\begin{array}{lll}0 & 0 & 1\end{array}\right]$} \\
\hline \multirow{3}{*}{ Neutra } & LDA & 2.25 & +8.17 & 0.00 & {$\left[\begin{array}{lll}1 & 1 & 1\end{array}\right]$} & $\begin{array}{l}2.13 \\
2.25 \\
2.33\end{array}$ & -4.40 & 0.53 & {$\left[\begin{array}{lll}1 & -0.7 & 0.7\end{array}\right]$} \\
\hline & $\begin{array}{l}\text { CW- } \\
\text { GGA }\end{array}$ & 2.25 & +8.32 & 0.00 & {$\left[\begin{array}{lll}1 & 1 & 1\end{array}\right]$} & $\begin{array}{l}2.14 \\
2.28 \\
2.32\end{array}$ & -3.81 & 0.34 & {$\left[\begin{array}{lll}1 & -0.7 & 0.7\end{array}\right]$} \\
\hline & GGA & 2.25 & +8.25 & 0.00 & {$\left[\begin{array}{lll}1 & 1 & 1\end{array}\right]$} & $\begin{array}{l}2.14 \\
2.28 \\
2.32\end{array}$ & -3.91 & 0.40 & {$\left[\begin{array}{lll}1 & -0.7 & 0\end{array}\right]$} \\
\hline \multirow{3}{*}{ Cargada } & LDA & 2.28 & +8.05 & 0.00 & [ $\left.\begin{array}{lll}1 & 1 & 1\end{array}\right]$ & $\begin{array}{l}2.17 \\
2.30 \\
2.31\end{array}$ & +7.37 & 0.67 & {$\left[\begin{array}{lll}0.8 & 1 & 0\end{array}\right]$} \\
\hline & $\begin{array}{l}\text { CW- } \\
\text { GGA }\end{array}$ & 2.28 & +8.16 & 0.00 & {$\left[\begin{array}{lll}1 & 1 & 1\end{array}\right]$} & $\begin{array}{l}2.19 \\
2.31 \\
2.32 \\
\end{array}$ & +6.50 & 0.71 & {$\left[\begin{array}{lll}0.8 & 1 & 0\end{array}\right]$} \\
\hline & GGA & 2.28 & +8.18 & 0.00 & {$\left[\begin{array}{lll}1 & 1 & 1\end{array}\right]$} & $\begin{array}{l}2.19 \\
2.32 \\
2.32\end{array}$ & +6.99 & 0.66 & {$\left[\begin{array}{lll}0.8 & 1 & 0\end{array}\right]$} \\
\hline
\end{tabular}

Tabla 7.20: Cálculos APW+lo de $d_{N N}, \mathrm{~V}_{33}$ (dirección y magnitud) y $\eta$ en función del estado de carga de la celda de $\mathrm{Sc}_{2} \mathrm{O}_{3}$ :Cd en las aproximaciones LDA, GGA y CW-GGA. Las distancias $d_{N N}$ están dadas en $\AA$ y $\mathrm{V}_{33}$ en unidades de $10^{21} \mathrm{~V} / \mathrm{m}^{2}$. 


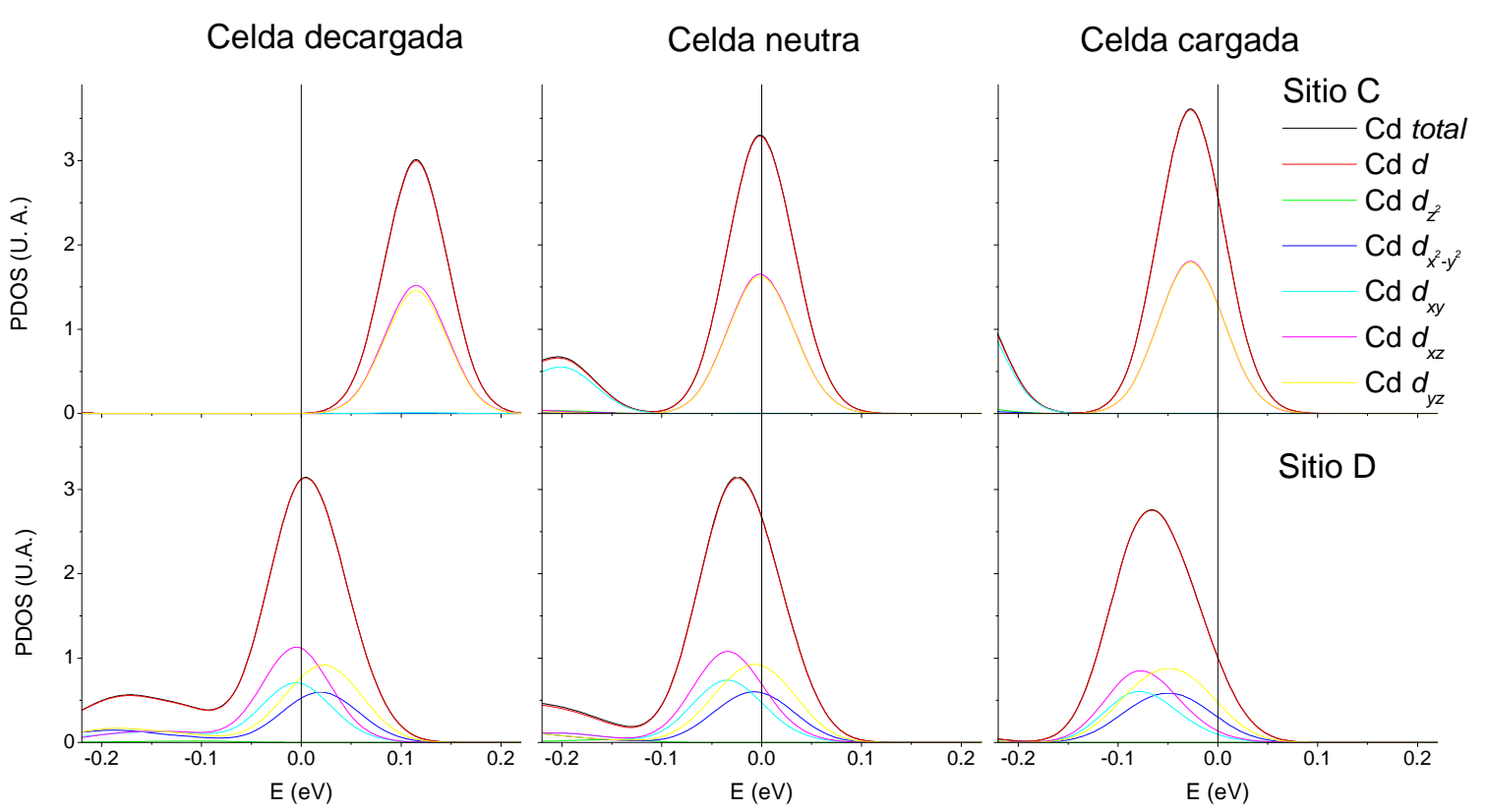

Figura 7.19: PDOS de las simetrías $d$ del $\mathrm{Cd}$ en $\mathrm{Sc}_{2} \mathrm{O}_{3}$. Las $\mathrm{PDOS}$ de la izquierda corresponden a la celda descargada, las del centro a la celda neutra y en la derecha se grafican las de celda cargada. Los gráficos superiores corresponden a Cd en sitio C y las PDOS inferiores a Cd en sitio D.

En la Figura 7.19 se grafican las simetrías $d$ del Cd para los tres estados de carga. El resultado obtenido es similar a las otras bixbitas. Para Cd en sitio D, en el llenado del nivel de impureza participan todas las componentes $d$ excepto $d_{\mathrm{z}^{2}}$ y para $\mathrm{Cd}$ en sitio C solo contribuyen las simetrías $d_{x z}$ y $d_{y z}$. Finalmente como en las otras bixbitas, la presencia de solo dos simetrías $d$ en el nivel de impureza observado para el caso del Cd en el sitio C implica una fuerte dependencia del GCE con el estado de carga, mientras que el comportamiento uniforme con las componentes $d$ observado en el Cd en el sitio D se relaciona con la independencia del GCE respecto al llenado o vaciado del nivel de impureza [Muñoz 2011].

Por último, analizaremos las contribuciones $p, d$ y $s$ - $d$ al GCE calculado dentro de la esfera del Cd. La Tabla 7.21 corresponde al GCE del Cd en sitio C y la Tabla 7.22 al Cd en el sitio D. Al igual que en las otras bixbitas, en general la contribución $p$ está por encima de la contribución $d$, la contribución $s$ - $d$ es despreciable frente a las otras. Nuevamente, de la comparación de la dependencia de las componentes $V_{i i}$ con el estado de carga de la celda se observa una fuerte dependencia en el caso de Cd en sitio C mientras que el GCE se vuelve independiente para el sitio D. 


\section{Cd en sitio C}

\begin{tabular}{cccccccccc}
\hline & \multicolumn{3}{c}{ Celda descargada } & \multicolumn{3}{c}{ Celda neutra } & \multicolumn{3}{c}{ Celda cargada } \\
\hline & $\boldsymbol{V}_{\mathbf{1 1}}$ & $\boldsymbol{V}_{\mathbf{2 2}}$ & $\boldsymbol{V}_{\mathbf{3 3}}$ & $\boldsymbol{V}_{\mathbf{1 1}}$ & $\boldsymbol{V}_{\mathbf{2 2}}$ & $\boldsymbol{V}_{\mathbf{3 3}}$ & $\boldsymbol{V}_{\mathbf{1 1}}$ & $\boldsymbol{V}_{\mathbf{2 2}}$ & $\boldsymbol{V}_{\mathbf{3 3}}$ \\
\hline $\boldsymbol{p}$ & +9.60 & -6.47 & -3.12 & -2.24 & +7.46 & -5.22 & -1.20 & -3.67 & +4.87 \\
\hline $\boldsymbol{d}$ & -10.32 & +4.20 & +6.12 & +3.46 & -4.86 & +1.39 & +0.25 & -2.03 & +1.78 \\
\hline $\boldsymbol{s}-\boldsymbol{d}$ & +0.17 & -0.21 & +0.05 & +0.04 & +0.15 & -0.19 & +0.02 & +0.13 & -0.15 \\
\hline total & -0.50 & -2.53 & +3.03 & +1.25 & +2.57 & -3.82 & -0.93 & -5.57 & +6.50 \\
\hline \hline
\end{tabular}

Tabla 7.21: Cálculos APW+lo de las contribuciones $p, d$ y s- $d$ al tensor GCE en función del estado de carga de la celda (aproximación CW-GGA) para Cd en sitio C del $\mathrm{Sc}_{2} \mathrm{O}_{3} . V_{i i}(i=1$, 2, 3) viene dado en unidades de $10^{21} \mathrm{~V} / \mathrm{m}^{2}$.

\section{Cd en sitio D}

\begin{tabular}{cccccccccc}
\hline & \multicolumn{3}{c}{ Celda descargada } & \multicolumn{3}{c}{ Celda neutra } & \multicolumn{3}{c}{ Celda cargada } \\
\hline & $\boldsymbol{V}_{\mathbf{1 1}}$ & $\boldsymbol{V}_{\mathbf{2 2}}$ & $\boldsymbol{V}_{\mathbf{3 3}}$ & $\boldsymbol{V}_{\mathbf{1 1}}$ & $\boldsymbol{V}_{\mathbf{2 2}}$ & $\boldsymbol{V}_{\mathbf{3 3}}$ & $\boldsymbol{V}_{\mathbf{1 1}}$ & $\boldsymbol{V}_{\mathbf{2 2}}$ & $\boldsymbol{V}_{\mathbf{3 3}}$ \\
\hline $\boldsymbol{p}$ & -3.34 & -3.34 & +6.68 & -3.18 & -3.18 & +6.36 & -3.00 & -3.00 & +6.00 \\
\hline $\boldsymbol{d}$ & -0.89 & -0.89 & +1.78 & -1.08 & -1.08 & +2.16 & -1.17 & -1.17 & +2.35 \\
\hline $\boldsymbol{s}-\boldsymbol{d}$ & +0.10 & +0.10 & -0.20 & +0.10 & +0.10 & -0.20 & +0.10 & +0.10 & -0.20 \\
\hline total & -4.13 & -4.13 & +8.26 & -4.16 & -4.16 & +8.32 & -4.07 & -4.07 & +8.15 \\
\hline \hline
\end{tabular}

Tabla 7.22: Cálculos APW+lo de las contribuciones $p, d$ y $s-d$ al tensor GCE en función del estado de carga de la celda (aproximación CW-GGA) para Cd en sitio D del $\mathrm{Sc}_{2} \mathrm{O}_{3} . V_{i i}(i=1$, 2, 3) viene dado en unidades de $10^{21} \mathrm{~V} / \mathrm{m}^{2}$.

\section{7-2-4 Cálculos APW+lo del tensor GCE en ZnO dopado con Cd}

En este apartado describiremos los resultados de los cálculos $a b$ initio realizados en el sistema ZnO:Cd. Para garantizar la dilución de la impureza en la red huésped se construyó una supercelda de 3 celdas unidad en la dirección de los ejes $a$ y $b$, y dos celdas unidad en la dirección del eje c (celda de 3x3x2). De este modo, los parámetros de red $a^{\prime}, b^{\prime}$ y $c^{\prime}$ de la supercelda de 3x3x2 del ZnO:Cd son $a^{\prime}=b^{\prime}=3 a=9.7503 \AA$ y $c^{\prime}=2 c=10.4142 \AA$. Se realizaron cálculos del tensor GCE en función del estado de carga 
de la celda con 4 estados de carga: el caso de celda neutra corresponde a reemplazar un átomo nativo ( $\mathrm{Zn}$ ) por una impureza $\mathrm{Cd}$ en la supercelda; el caso de celda cargada corresponde a agregar un electrón a la celda neutra; y los casos de celda descargada y descargada con " $2 e^{-» "}$ corresponden a remover 1 y 2 electrones de la celda neutra, respectivamente.

En la Figura 7.20 se grafican las DOS del monóxido de zinc puro y dopado con Cd correspondientes a los tres estados de carga: neutra, descargada y cargada. Desde la comparación de las DOS correspondientes a ZnO puro (Fig. 7.20 (d)) y dopado (Fig. 7.20 (a), (b) y (c)) se aprecia que la inclusión de la impureza Cd en la red del ZnO introduce estados de impureza muy apaisados y cercano al fondo de la banda de conducción evidenciando el carácter isovalente que presenta el ión de impureza $\mathrm{Cd}^{2+}$ al reemplazar al ión nativo $\mathrm{Zn}^{2+}$.

Como se puede apreciar en todas las DOS de la Fig. 7.20, la banda de valencia presenta una contribución mayoritaria de las esferas de los oxígenos. Además, la contribución de los cationes Zn resulta muy apreciable en dicha banda a diferencia de lo que sucede en las bixbitas (ver sección anterior) donde la contribución de los cationes a la banda de valencia resultaba despreciable frente a los oxígenos. Este resultado se correlaciona con el tipo de estructura cristalina: en el caso de las bixbitas la estructura es más compacta debido al carácter mayoritariamente iónico de los enlaces (contribución despreciable de los cationes a la banda de valencia) mientras que en el ZnO su estructura tetraédrica es más abierta y los enlaces presentan un carácter más covalente (contribución muy apreciable de los cationes a la banda de valencia). Por otro lado, se observa que el Cd no introduce niveles de impureza pronunciados en el gap del semiconductor a diferencia de lo que sucede en las bixbitas donde dicha impureza introduce un nivel simple aceptor en el tope de la banda de valencia. Este resultado (en el $\mathrm{ZnO}: \mathrm{Cd}$ ) se atribuye al carácter isovalente que presenta el $\mathrm{Cd}^{2+}$ con el $\mathrm{Zn}^{2+}$ en contraposición a los sesquióxidos de In, Sc e Y donde los cationes actúan con valencia $3+$. 
(a)

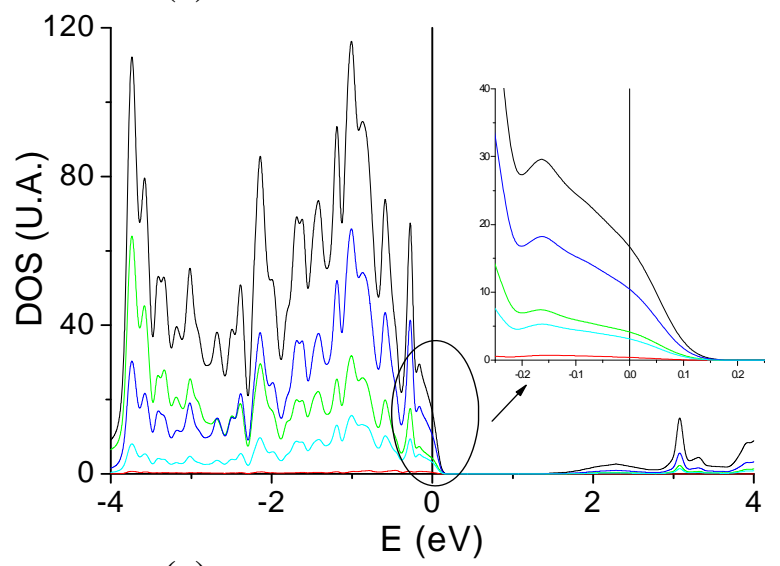

(c)

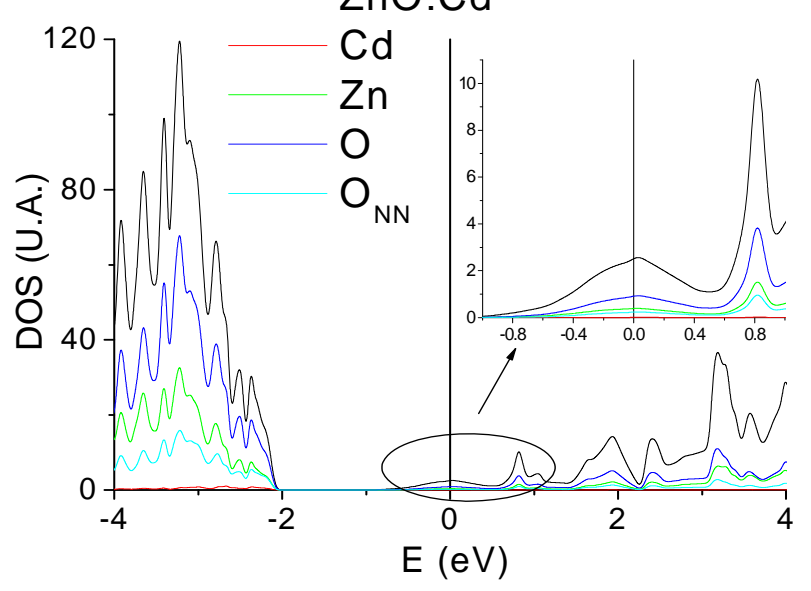

(b)

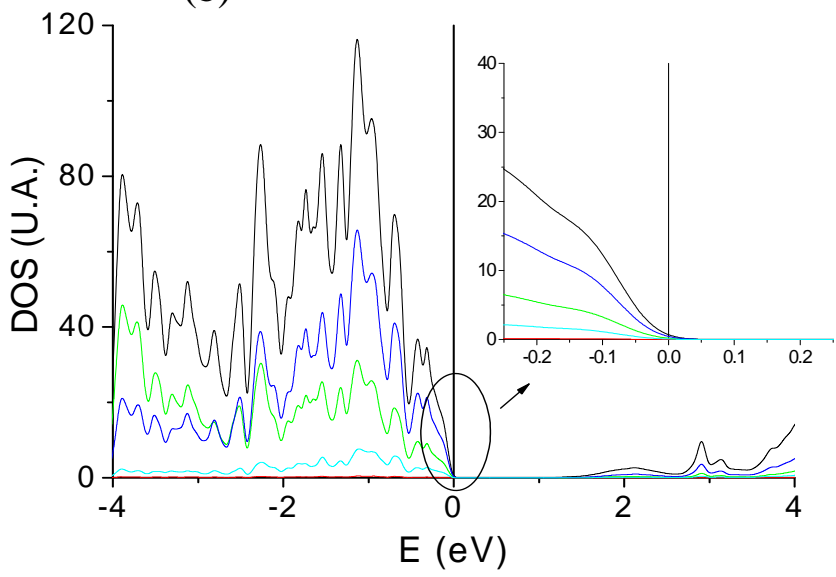

(d)

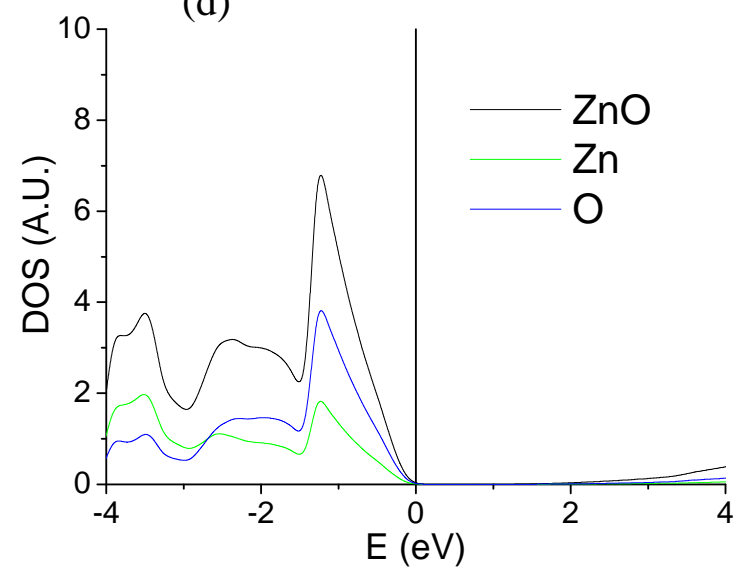

Figura 7.20: DOS del óxido $\mathrm{ZnO}$ puro y dopado con $\mathrm{Cd}$. Además se representan las contribuciones de las esferas atómicas de $\mathrm{Zn}, \mathrm{O}, \mathrm{Cd}$ y los vecinos $\mathrm{O}_{\mathrm{NN}}$. Se grafican las DOS correspondientes a los siguientes casos: (a) celda de $\mathrm{ZnO}: \mathrm{Cd}$ descargada; (b) celda de ZnO:Cd neutra; (c) celda ZnO:Cd cargada; (d) ZnO puro.

Desde la comparación de las DOS correspondientes a los distintos estados de carga, se observa que al agregar un electrón a la ceda descargada (Fig. 7.20 (a)), la banda de valencia se llena totalmente (Fig. 7.20 (b)). Mientras que al agregar un electrón a la celda neutra (Fig. 7.20 (b)), el fondo de la banda de conducción se ocupa parcialmente (Fig. 7.20 (c)). 


\begin{tabular}{|c|c|c|c|c|c|}
\hline $\begin{array}{c}\text { Estados de carga } \\
\text { de la celda }\end{array}$ & Aprox. & $d_{N N}(\AA)$ & $\eta$ & $V_{33}\left(10^{21} V / m^{2}\right)$ & Dir. $V_{33}$ \\
\hline Descargada 2e- & CW-GGA & $\begin{array}{l}2.16 \\
2.19 \\
\end{array}$ & 0.00 & +1.20 & {$\left[\begin{array}{lll}0 & 0 & 1\end{array}\right]$} \\
\hline \multirow{3}{*}{ Descargada } & LDA & $\begin{array}{l}2.16 \\
2.18 \\
\end{array}$ & 0.00 & +1.07 & {$\left[\begin{array}{lll}0 & 0 & 1\end{array}\right]$} \\
\hline & CW-GGA & $\begin{array}{l}2.16 \\
2.19 \\
\end{array}$ & 0.00 & +1.37 & {$\left[\begin{array}{lll}0 & 0 & 1\end{array}\right]$} \\
\hline & GGA & $\begin{array}{l}2.16 \\
2.19 \\
\end{array}$ & 0.00 & +1.20 & {$\left[\begin{array}{lll}0 & 0 & 1\end{array}\right]$} \\
\hline \multirow{3}{*}{ Neutra } & LDA & $\begin{array}{l}2.16 \\
2.18 \\
\end{array}$ & 0.00 & +1.43 & {$\left[\begin{array}{lll}0 & 0 & 1\end{array}\right]$} \\
\hline & CW-GGA & $\begin{array}{l}2.16 \\
2.19\end{array}$ & 0.00 & +1.68 & {$\left[\begin{array}{lll}0 & 0 & 1\end{array}\right]$} \\
\hline & GGA & $\begin{array}{l}2.16 \\
2.19\end{array}$ & 0.00 & +1.69 & {$\left[\begin{array}{lll}0 & 0 & 1\end{array}\right]$} \\
\hline \multirow{3}{*}{ Cargada } & LDA & $\begin{array}{l}2.16 \\
2.18 \\
\end{array}$ & 0.00 & +1.30 & {$\left[\begin{array}{lll}0 & 0 & 1\end{array}\right]$} \\
\hline & CW-GGA & $\begin{array}{l}2.16 \\
2.19\end{array}$ & 0.00 & +1.79 & {$\left[\begin{array}{lll}0 & 0 & 1\end{array}\right]$} \\
\hline & GGA & $\begin{array}{l}2.16 \\
2.19\end{array}$ & 0.00 & +1.78 & {$\left[\begin{array}{lll}0 & 0 & 1\end{array}\right]$} \\
\hline
\end{tabular}

Tabla 7.23: Cálculos APW+lo de $d_{N N}, \mathrm{~V}_{33}$ (dirección y magnitud) y $\eta$ en función del estado de carga de la celda de ZnO:Cd en las aproximaciones LDA, GGA y CW-GGA. Las distancias $d_{N N}$ están dadas en $\AA$ y $\mathrm{V}_{33}$ en unidades de $10^{21} \mathrm{~V} / \mathrm{m}^{2}$.

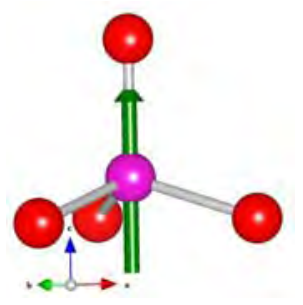

Figura 7.21: Dirección de $\mathrm{V}_{33}$ en el sitio del Cd en $\mathrm{ZnO}$.

En la Tabla 7.23 se detallan los parámetros que caracterizan al GCE en función del estado de carga de la supercelda. Se aprecia un excelente acuerdo entre las predicciones realizadas en las aproximaciones CW-GGA y GGA mientras los resultados obtenidos con LDA difieren en un $20 \%$ con las otras dos aproximaciones para el caso de celda descargada y neutra, y en un 30\% para el caso de la celda cargada. En cuanto a la dependencia del GCE con el estado de carga de la celda en la aproximación CW-GGA, se observa que la magnitud de la componente principal $\mathrm{V}_{33}$ aumenta menos del $10 \%$ cuando un electrón es agregado a la celda neutra, mientras que al remover uno y dos electrones de la celda neutra $\mathrm{V}_{33}$ cambia en un $20 \%$ y 30\%, respectivamente. Por otro lado, la dirección de $\mathrm{V}_{33}$ no presenta cambios con el estado de carga y el parámetro $\eta$ es mantenido en cero por el método de cálculo debido a que preserva la simetría puntual del sitio catiónico de la impureza en el proceso de relajación de la estructura. 
En la Figura 7.22 se grafican las densidades parciales PDOS para las simetrías $s, p$ y $d$ del Cd en la región cercana a la energía de Fermi. Se puede observar que en la banda de valencia la contribución es prácticamente de carácter $d$, mientras que el fondo de la banda de conducción presenta una débil contribución $s$ y $d$. Para analizar las diferentes simetrías $d$ que aportan a la banda de valencia, en la Figura 7.23 se grafican las simetrías $d$ del Cd en la región del nivel de Fermi en función del estado de carga de la supercelda. Se puede apreciar que en los casos donde el nivel de Fermi se encuentra en la banda de valencia (celda neutra y descargada con 1 y 2 electrones menos), la simetría $d$ del Cd contribuye apreciablemente a la PDOS mientras que en el caso de la celda cargada la contribución $d$ en particular, y del Cd en general, es despreciable. Es por esto que la mayor dependencia del GCE con el estado de carga del sistema se observa al remover uno y dos electrones de la celda neutra, mientras que al agregarle un electrón el GCE se ve modificado en menos del $10 \%$.

Finalmente, analizaremos las diferentes contribuciones $p, d$ y $s-d$ al GCE y su dependencia con el estado de carga de la supercelda. En la Tabla 7.24 se muestran estos resultados. Como puede apreciarse, al igual que lo observado en las bixbitas, las contribuciones $p$ de las tres componentes $\mathrm{V}_{\text {ii }}$ están por encima de las contribuciones $d$. Además, las contribuciones $s$ - $d$ resultan nulas. Finalmente, se observa que las contribuciones $p$ al GCE aumentan a medida que se agregan electrones comenzando desde la supercelda más descargada, mientras que las contribuciones $d$ solo son apreciables en los casos descargados con 1 y 2 electrones menos. Esto se debe a que la contribución $d$ a la PDOS solo es apreciable en los casos de celda descargada con 1 y 2 electrones.

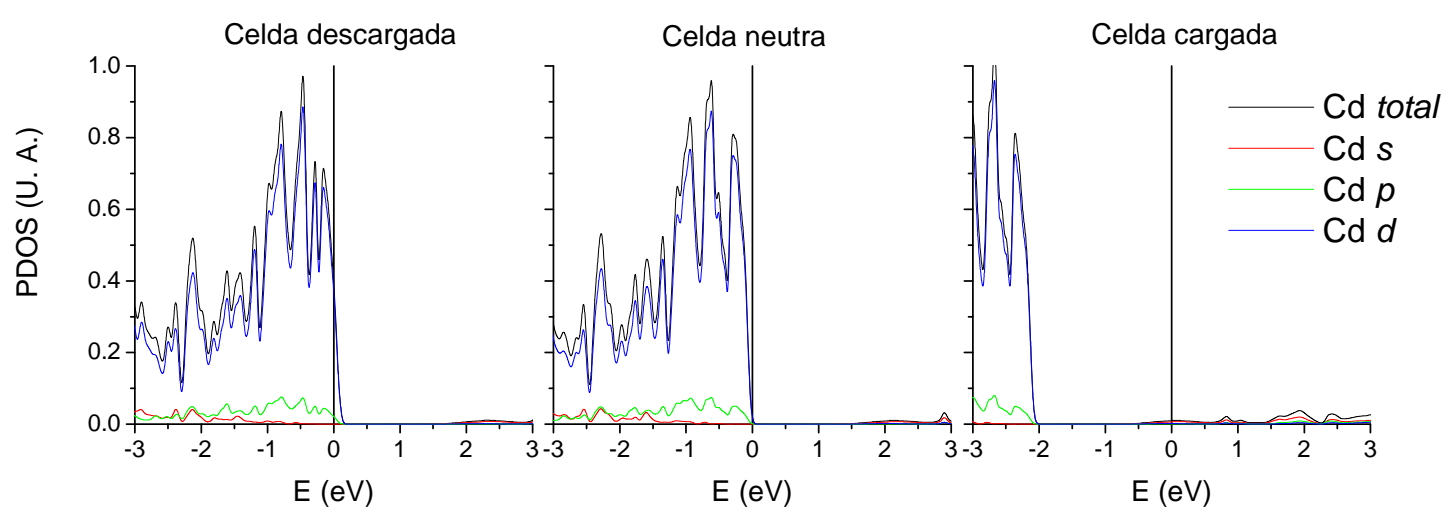

Figura 7.22: PDOS de las simetrías $s, p$ y $d$ de la impureza Cd en el óxido ZnO. De izquierda a derecha se muestran las densidades en la región del nivel de Fermi en celda la descargada, neutra y cargada, respectivamente. 


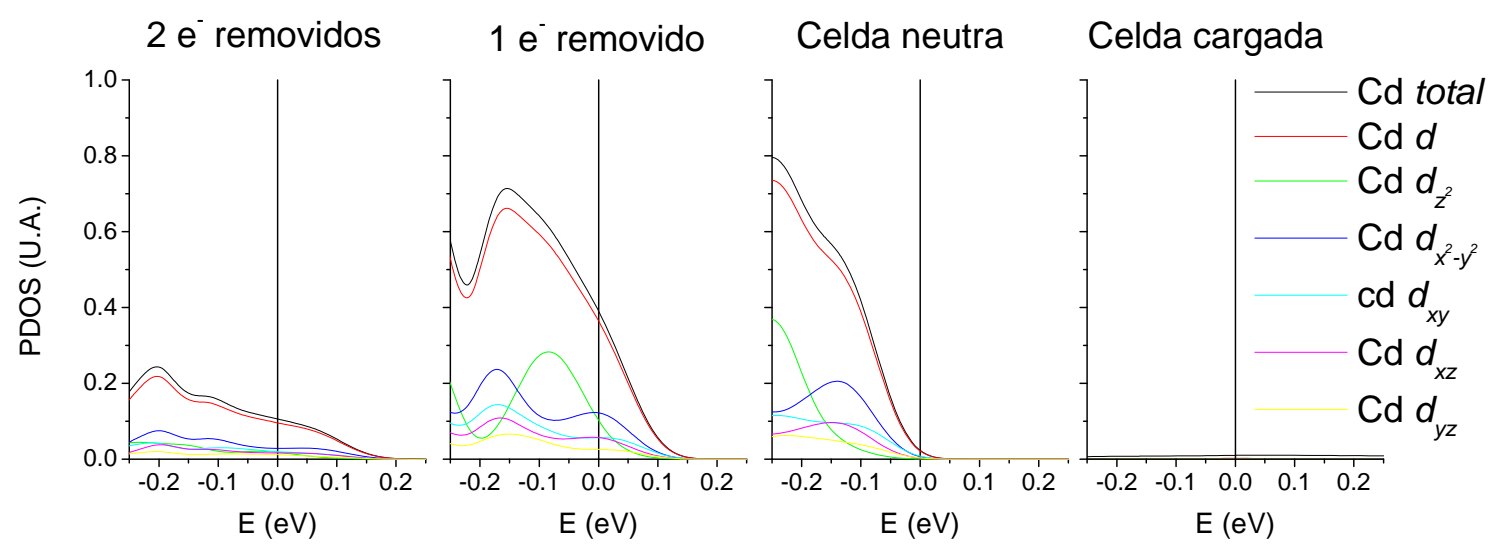

Figura 7.23: PDOS de las simetrías $d$ del Cd del sistema $\mathrm{ZnO}: \mathrm{Cd}$. De izquierda a derecha se muestran las densidades en la región del nivel de Fermi comenzando con celda descargada con $2 e^{-}$(izquierda) hasta celda cargada (derecha), respectivamente.

\begin{tabular}{ccccccccccccc}
\hline \hline & \multicolumn{2}{c}{ Descargada con $2 \mathbf{e}^{-}$} & \multicolumn{2}{c}{ Celda descargada } & \multicolumn{2}{c}{ Celda neutra } & \multicolumn{3}{c}{ Celda cargada } \\
\hline & $\mathbf{V}_{\mathbf{1 1}}$ & $\mathbf{V}_{\mathbf{2 2}}$ & $\mathbf{V}_{\mathbf{3 3}}$ & $\mathbf{V}_{\mathbf{1 1}}$ & $\mathbf{V}_{\mathbf{2 2}}$ & $\mathbf{V}_{\mathbf{3 3}}$ & $\mathbf{V}_{\mathbf{1 1}}$ & $\mathbf{V}_{\mathbf{2 2}}$ & $\mathbf{V}_{\mathbf{3 3}}$ & $\mathbf{V}_{\mathbf{1 1}}$ & $\mathbf{V}_{\mathbf{2 2}}$ & $\mathbf{V}_{\mathbf{3 3}}$ \\
\hline $\boldsymbol{p}$ & -0.73 & -0.73 & +1.46 & -0.79 & -0.79 & +1.59 & -0.86 & -0.86 & +1.72 & -0.88 & -0.88 & +1.76 \\
\hline $\boldsymbol{d}$ & -0.14 & +0.14 & -0.28 & +0.11 & +0.11 & -0.22 & +0.02 & +0.02 & -0.03 & +0.01 & +0.01 & -0.02 \\
\hline $\boldsymbol{s}-\boldsymbol{d}$ & 0.00 & 0.00 & 0.00 & 0.00 & 0.00 & 0.00 & 0.00 & 0.00 & 0.00 & 0.00 & 0.00 & 0.00 \\
\hline total & -0.59 & -0.59 & +1.18 & -0.68 & -0.68 & +1.36 & -0.84 & -0.84 & +1.68 & -0.87 & -0.87 & +1.74 \\
\hline \hline
\end{tabular}

Tabla 7.24: Cálculos APW+lo de las contribuciones $p, d$ y s- $d$ al tensor GCE en función del estado de carga de la celda (aproximación CW-GGA) para Cd en $\mathrm{ZnO}$. $V_{i i}(i=1,2,3)$ viene dado en unidades de $10^{21} \mathrm{~V} / \mathrm{m}^{2}$.

\section{7-2-5 Cálculos APW+lo del tensor GCE en $\mathrm{SnO}_{2}$ dopado con Cd}

En esta sección se muestran los cálculos APW+lo correspondientes al óxido de $\mathrm{SnO}_{2}$ con estructura rutilo dopado con Cd. Para garantizar la dilución de la impureza en el sistema huésped se construyó una supercelda formada por 2 celdas unidad en la dirección de los ejes $a$ y $b$, y 3 celdas unidad en la dirección del eje $c$ (supercelda de 2x2x3). Por lo tanto, los parámetros de red $a^{\prime}, b^{\prime}$ y $c^{\prime}$ de la supercelda son $a^{\prime}=b^{\prime}=2 a=9.4748 \AA$ y $c^{\prime}=3 c=9.5592 \AA$. Se realizaron cálculos del GCE en función del estado de carga de la supercelda en los siguientes estados de carga: celda neutra, 
reemplazando un átomo nativo (Sn) por una impureza $\mathrm{Cd}$; celda semicargada, agregando un electrón a la celda neutra; y celda cargada, agregando 2 electrones a la celda neutra.

En la Figura 7.24 se grafican las DOS del $\mathrm{SnO}_{2}$ puro y dopado con Cd, además se incluyen las contribuciones de las esferas de los átomos Sn, de los oxígenos para ambos casos, y adicionalmente, las contribuciones de la impureza Cd y de los primeros vecinos $\mathrm{O}_{\mathrm{NN}}$ en el caso dopado. Para el caso dopado se grafican las DOS correspondientes a los 3 estados de carga: celda neutra, celda semicargada y celda cargada (Fig. 7.24 (a), (b) y (c), respectivamente). Desde la comparación de las DOS correspondientes al caso puro (Fig. 7.24 (d)) y dopado (Fig. 7.24 (a), (b) y (c)), se aprecia que la inclusión de la impureza Cd en la red del $\mathrm{SnO}_{2}$ introduce un nivel de impureza doble aceptor en el tope de la banda de valencia del semiconductor. De hecho la integración de los estados electrónicos que están por encima del nivel de Fermi de la celda neutra (Fig. 7.24 (a)) da como resultado 2 electrones.

Desde los gráficos de las DOS se puede ver que la banda de valencia del semiconductor es prácticamente igual a la integral de la densidad de estados en las esferas de los oxígenos, mientras que la contribución de las esferas de Sn en dicha banda se vuelve despreciable. Esto muestra que el semiconductor $\mathrm{SnO}_{2}$ presenta un carácter esencialmente iónico como en el caso de las bixbitas y se correlaciona con su estructura más compacta respecto a la del ZnO de carácter más covalente.

Finalmente, de la integración en las esferas atómicas “filtrando” las energías de los estados que contribuyen al nivel de impureza sobre el nivel de Fermi se obtuvo que para el caso de celda neutra los oxígenos contribuyen con 74\% al nivel, el Cd con un $12 \%$, los Sn con (1\%) y los vecinos $\mathrm{O}_{\mathrm{NN}}$ del Cd con un 41\%. En el caso de celda semicargada, las esferas de O aportan 69\%, el Cd contribuye con un 15\%, los Sn con 2\% y los primeros vecinos $\mathrm{O}_{\mathrm{NN}}$ aportan un $49 \%$. Y en la celda cargada, los oxígenos contribuyen con $65 \%$, la impureza Cd con 19\%, los cationes Sn con 2\% y los $\mathrm{O}_{\mathrm{NN}}$ aportan un 51\%. Por lo tanto, de la integración en las esferas en el rango energético de los estados del nivel de impureza se deduce que esencialmente dichos estados electrónicos se encuentran en el entorno de la impureza $\mathrm{Cd}$ y sus vecinos $\mathrm{O}_{\mathrm{NN}} \mathrm{y}$ este resultado ha sido observado para los tres estados de carga. 
(a)

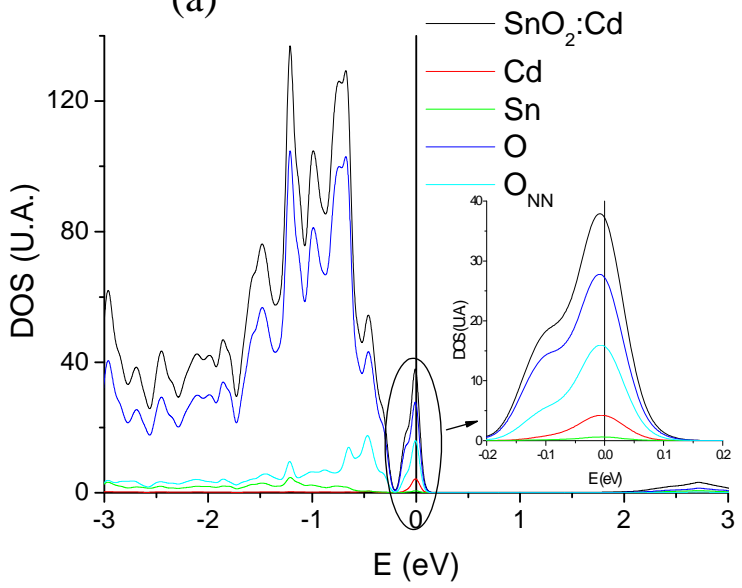

(c)

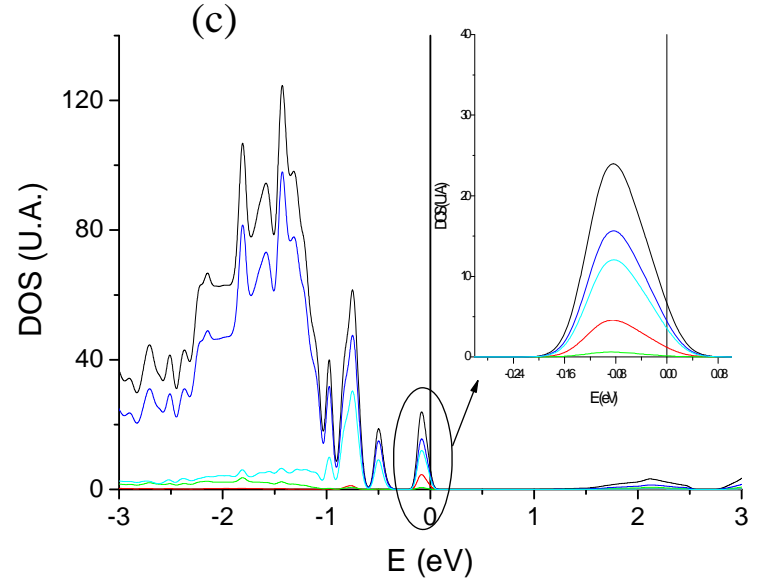

(b)

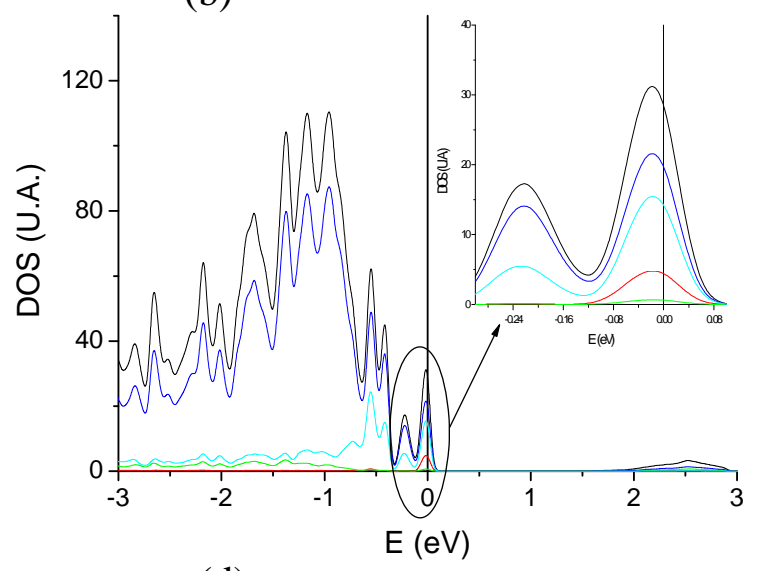

(d)

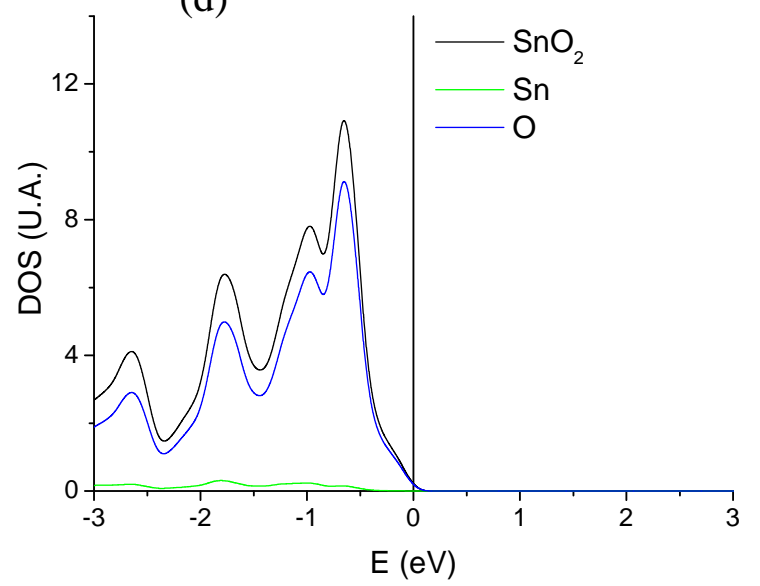

Figura 7.24: Densidades de estados (DOS) del óxido $\mathrm{SnO}_{2}$ puro y dopado con Cd con las contribuciones de las esferas atómicas de $\mathrm{Zn}, \mathrm{O}$, Cd y los vecinos $\mathrm{O}_{\mathrm{NN}}$. Se grafican las DOS correspondientes a los siguientes casos: (a) celda de $\mathrm{SnO}_{2}$ :Cd neutra; (b) celda de $\mathrm{SnO}_{2}: \mathrm{Cd}$ semicargada; (c) celda de $\mathrm{SnO}_{2}$ :Cd cargada; y (d) $\mathrm{SnO}_{2}$ puro.

En la Tabla 7.25 se detalla la dependencia del GCE con el estado de carga de la supercelda calculadas en las aproximaciones LDA, GGA y CW-GGA. De la comparación entre las aproximaciones se observa que los resultados obtenidos con GGA y CW-GGA están en muy buen acuerdo, mientras que LDA presenta discrepancias frente a las predicciones GGA y CW-GGA menores al 10\% tanto para $\mathrm{V}_{33}$ como para $\eta$ excepto en el caso de celda neutra donde difiere en un $15 \%$ y un $23 \%$, respectivamente. En cuanto a la dependencia con el estado de carga, se observa que al agregar un electrón a la celda neutra el parámetro de asimetría y la componente principal $\mathrm{V}_{33}$ aumentan en un 20\% aproximadamente (en las aproximaciones GGA y CW-GGA). Al agregar el segundo electrón, el parámetro $\eta$ se reduce en un 30\% mientras que $\mathrm{V}_{33}$ cambia en menos del 5\% (también en las aproximaciones GGA y CW- 
GGA). Por último, se aprecia que la dirección de $V_{33}$ no se ve modificada con el cambio en el estado de carga de la celda mientras que la distancia entre primeros vecinos Cd$\mathrm{O}_{\mathrm{NN}}$ aumenta con el agregado de electrones a la supercelda debido al efecto de la repulsión coulombiana.

\begin{tabular}{|c|c|c|c|c|c|}
\hline $\begin{array}{l}\text { Estados de carga } \\
\text { de la celda }\end{array}$ & Aprox. & $d_{N N}(\AA)$ & $\eta$ & $V_{33}\left(10^{21} \mathrm{~V} / \mathrm{m}^{2}\right)$ & Dir. $V_{33}$ \\
\hline \multirow{3}{*}{ Neutra } & LDA & $\begin{array}{l}2.11 \\
2.13\end{array}$ & 0.49 & +5.19 & {$\left[\begin{array}{lll}1 & -1 & 0\end{array}\right]$} \\
\hline & CW-GGA & $\begin{array}{l}2.10 \\
2.14 \\
\end{array}$ & 0.34 & +5.02 & {$\left[\begin{array}{lll}1 & -1 & 0\end{array}\right]$} \\
\hline & GGA & $\begin{array}{l}2.10 \\
2.14 \\
\end{array}$ & 0.26 & +4.77 & {$\left[\begin{array}{lll}1 & -1 & 0\end{array}\right]$} \\
\hline \multirow{3}{*}{ Semi-Cargada } & LDA & $\begin{array}{l}2.13 \\
2.16 \\
\end{array}$ & 0.56 & +5.93 & {$\left[\begin{array}{lll}1 & -1 & 0\end{array}\right]$} \\
\hline & CW-GGA & $\begin{array}{l}2.12 \\
2.16\end{array}$ & 0.49 & +5.86 & {$\left[\begin{array}{lll}1 & -1 & 0\end{array}\right]$} \\
\hline & GGA & $\begin{array}{l}2.12 \\
2.16 \\
\end{array}$ & 0.55 & +6.17 & {$\left[\begin{array}{lll}1 & -1 & 0\end{array}\right]$} \\
\hline \multirow{3}{*}{ Cargada } & LDA & $\begin{array}{l}2.15 \\
2.20\end{array}$ & 0.14 & +5.47 & {$\left[\begin{array}{lll}1 & -1 & 0\end{array}\right]$} \\
\hline & CW-GGA & $\begin{array}{l}2.15 \\
2.20\end{array}$ & 0.21 & +5.73 & {$\left[\begin{array}{lll}1 & -1 & 0\end{array}\right]$} \\
\hline & GGA & $\begin{array}{l}2.15 \\
2.20\end{array}$ & 0.25 & +5.79 & {$\left[\begin{array}{lll}1 & -1 & 0\end{array}\right]$} \\
\hline
\end{tabular}

Tabla 7.25: Cálculos APW+lo de $d_{N N}, V_{33}$ (dirección y magnitud) y $\eta$ en función del estado de carga de la celda en las aproximaciones LDA y CW-GGA en $\mathrm{SnO}_{2}$ :Cd. Las distancias $d_{N N}$ están dadas en $\AA$ y $\mathrm{V}_{33}$ en unidades de $10^{21} \mathrm{~V} / \mathrm{m}^{2}$.

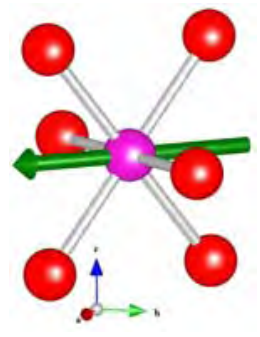

Figura 7.25:

Dirección de $\mathrm{V}_{33}$ en el sitio del Cd en $\mathrm{SnO}_{2}$.

En la Figura 7.26 se grafican las PDOS de las simetrías $d$ del Cd en el nivel de impureza y toda la PDOS de toda la esfera del Cd. Se puede observar que la contribución del Cd al nivel de impureza es prácticamente de simetría $d$. Además, se puede apreciar en la figura que sólo las simetrías $d_{z^{2}}, d_{x^{2}-y^{2}}$ y $d_{x z}$ contribuyen al llenado del nivel, esto se correlaciona con los cambios observados en el GCE con el agregado de electrones a la supercelda. 


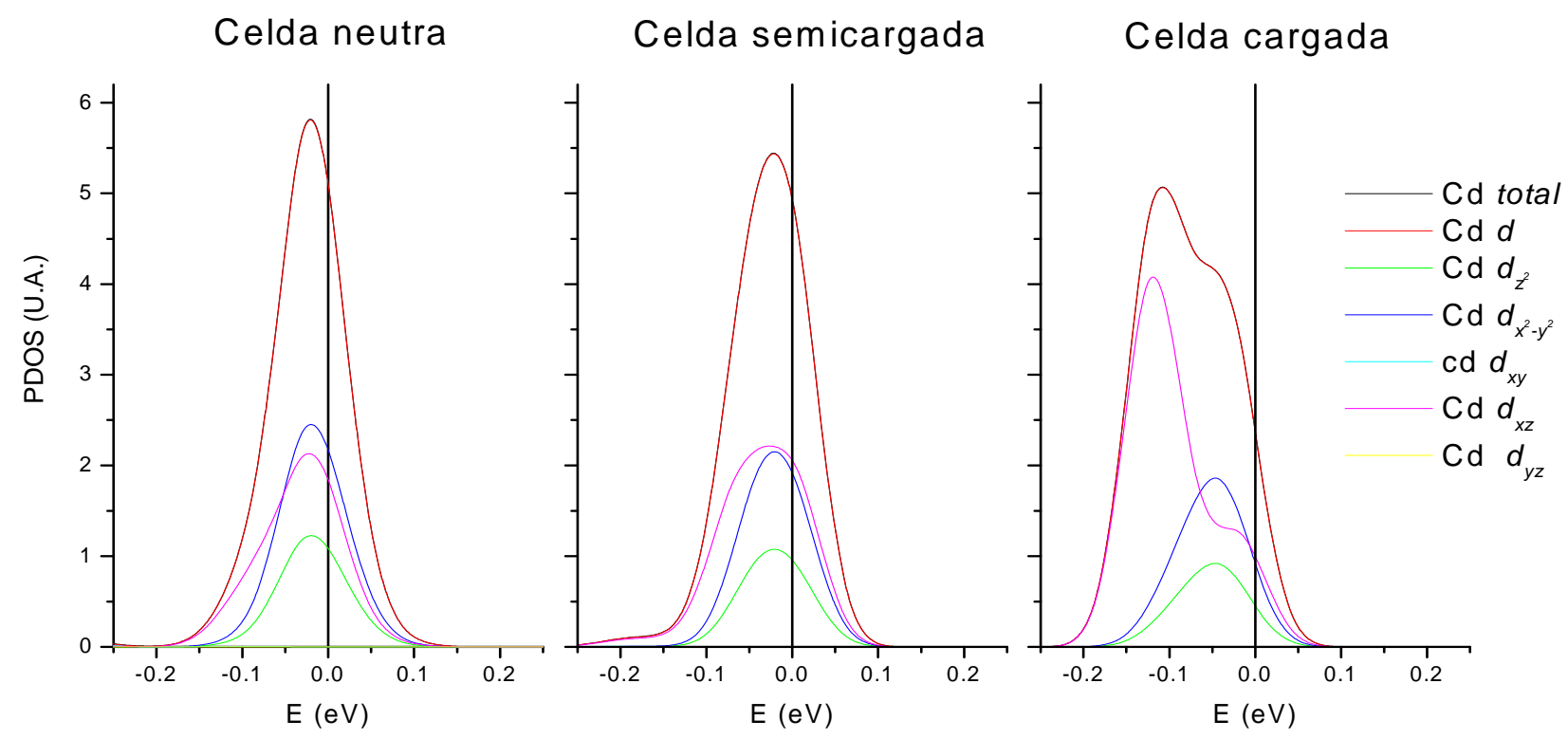

Figura 7.26: PDOS de las simetrías $d$ del $\mathrm{Cd}$ del sistema $\mathrm{SnO}_{2}: \mathrm{Cd}$. De izquierda a derecha se muestran las densidades en la región del nivel de Fermi en celda neutra, semicargada y cargada, respectivamente.

Finalmente, en la Tabla 7.26 se detallan las contribuciones $p$, $d$ y s- $d$ al tensor GCE en función del estado de carga. Al igual que en los otros óxidos se aprecia que las conntribuciones $p$ están por encima de las contribuciones $d$. Además, las componentes de simetría $p$ presentan cambios en sus magnitudes que no superan $20 \%$ de su valor al agregar electrones a la celda neutra, mientras que en el caso de las componentes del tipo $d$ cambian en signo y magnitud al agregar cargas negativas a la celda neutra. Estos cambios observados en las contribuciones $d$ son los responsables de la dependencia de las componentes totales con el estado de carga de la celda. Por último, las contribuciones de simetría $s$ - $d$ se vuelven despreciables frente a las otras simetrías. 


\begin{tabular}{cccccccccc}
\hline & \multicolumn{3}{c}{ Celda neutra } & \multicolumn{3}{c}{ Celda semi-cargada } & \multicolumn{3}{c}{ Celda cargada } \\
\hline & $\mathbf{V}_{\mathbf{1 1}}$ & $\mathbf{V}_{\mathbf{2 2}}$ & $\mathbf{V}_{\mathbf{3 3}}$ & $\mathbf{V}_{\mathbf{1 1}}$ & $\mathbf{V}_{\mathbf{2 2}}$ & $\mathbf{V}_{\mathbf{3 3}}$ & $\mathbf{V}_{\mathbf{1 1}}$ & $\mathbf{V}_{\mathbf{2 2}}$ & $\mathbf{V}_{\mathbf{3 3}}$ \\
\hline $\boldsymbol{p}$ & -2.89 & -2.67 & +5.55 & -3.11 & -1.88 & +4.99 & -2.34 & -2.53 & +4.87 \\
\hline $\boldsymbol{d}$ & +0.96 & -0.24 & -0.72 & +1.98 & -3.80 & +1.82 & +0.08 & -1.15 & +1.07 \\
\hline $\boldsymbol{s}-\boldsymbol{d}$ & +0.06 & +0.07 & -0.13 & +0.05 & +0.07 & -0.13 & +0.06 & +0.07 & -0.13 \\
\hline total & -1.86 & -2.85 & +4.72 & -1.07 & -5.62 & +6.69 & -2.21 & -3.61 & 5.82 \\
\hline \hline
\end{tabular}

Tabla 7.26: Cálculos APW+lo de las contribuciones $p, d$ y $s-d$ al tensor GCE en función del estado de carga de la celda (aproximación CW-GGA) para Cd en $\mathrm{SnO}_{2} . V_{i i}(i=1,2,3)$ viene dado en unidades de $10^{21} \mathrm{~V} / \mathrm{m}^{2}$.

\section{7-2-6 Cálculos APW+lo del tensor GCE en SnO dopado con Cd}

En esta sección analizaremos los cálculos APW+lo realizados en el sistema SnO dopado con Cd. Para considerar la correcta dilución de la impureza en el semiconductor se reemplazó un Sn por un Cd en una supercelda construida con 3 celdas unidad de SnO en la dirección del eje $a$, 3 celdas en la dirección del eje $b$ y 2 celdas en la dirección del eje $c$ (supercelda de $3 \times 3 \times 2$ ). De este modo, los parámetros de red de la supercelda son $a^{\prime}=b^{\prime}=3 a=11.4087 \AA$ y $c^{\prime}=2 c=9.6764 \AA$. En este sistema se realizaron cálculos con 3 estados de carga de la celda: celda neutra, reemplazando un átomo de Sn por una impureza Cd; celda descargada, removiendo un electrón de la celda neutra ; y celda cargada, agregando un electrón a la celda neutra.

En la Figura 7.27 se grafican las DOS correspondientes al sistema SnO puro y SnO:Cd para los tres estados de carga. Desde la comparación de las DOS para los casos puro (Fig. 7.27 (d)) y dopado (Fig. 7.27 (a), (b) y (c)) se observa que la inclusión de la impureza Cd en el semiconductor introduce niveles de impureza en el tope de la banda de valencia y en el fondo de la banda de conducción. Este hecho se relaciona con el carácter de isovalente del ión $\mathrm{Cd}^{2+}$ al reemplazar al ión nativo $\mathrm{Sn}^{2+}$ en el $\mathrm{SnO}$. Cabe destacar que si bien en el ZnO la impureza Cd también es isovalente, la diferencia en la forma de los picos de impureza (en el caso de SnO son mucho más pronunciados que en $\mathrm{ZnO}$ ) se relaciona con la localización de los estados de impureza en el semiconductor. En el capítulo siguiente (Cap. 8) se verá que la forma apaisada de los estados de impureza se correlaciona con estados altamente deslocalizados, mientras que picos bien pronunciados describen estados electrónicos localizados. Pero a diferencia de los 
niveles de impureza en el gap del semiconductor introducidos por la impureza Cd en las bixbitas o el dióxido de estaño (el Cd actúa como simple y doble aceptor en estos casos, respectivamente), donde los niveles presentan mayoritariamente contribuciones de los vecinos $\mathrm{O}_{\mathrm{NN}}$ y del Cd, en el caso de $\mathrm{SnO}: \mathrm{Cd}$ el nivel en el tope de la banda de valencia no presenta una apreciable contribución de la impureza y de los $\mathrm{O}_{\mathrm{NN}}$, mientras que en el nivel de impureza del fondo de la banda de conducción se aprecia una débil contribución del Cd y los $\mathrm{O}_{\mathrm{NN}}$ frente a la altura total del pico. En el siguiente capítulo veremos que esta débil contribución del Cd y sus vecinos $\mathrm{O}_{\mathrm{NN}}$ al nivel de impureza del fondo de la banda de conducción se debe a que estos estados electrónicos se localizan parcialmente en el entorno de la impureza y sus primeros vecinos oxígeno.

Desde las DOS representadas se puede observar que la contribución principal a la banda de valencia corresponde a los oxígenos. Sin embargo, la banda de valencia presenta una contribución muy apreciable de los cationes Sn como en el caso del ZnO, el cual presenta una estructura cristalina abierta. Cabe destacar que un resultado similar ha sido publicado recientemente en el sistema ZnO:Ta [Muñoz 2011].

Desde la comparación de las DOS para los distintos estados de carga se observa que al agregar un electrón a la celda descargada (Fig. 7.27 (a)) la banda de valencia se llena totalmente (Fig. 7.27 (b)). Mientras que al agregar un electrón a la celda neutra (Fig. 7.27 (b)) se ocupa parcialmente el fondo de la banda de conducción (Fig. 7.27 (c)). 

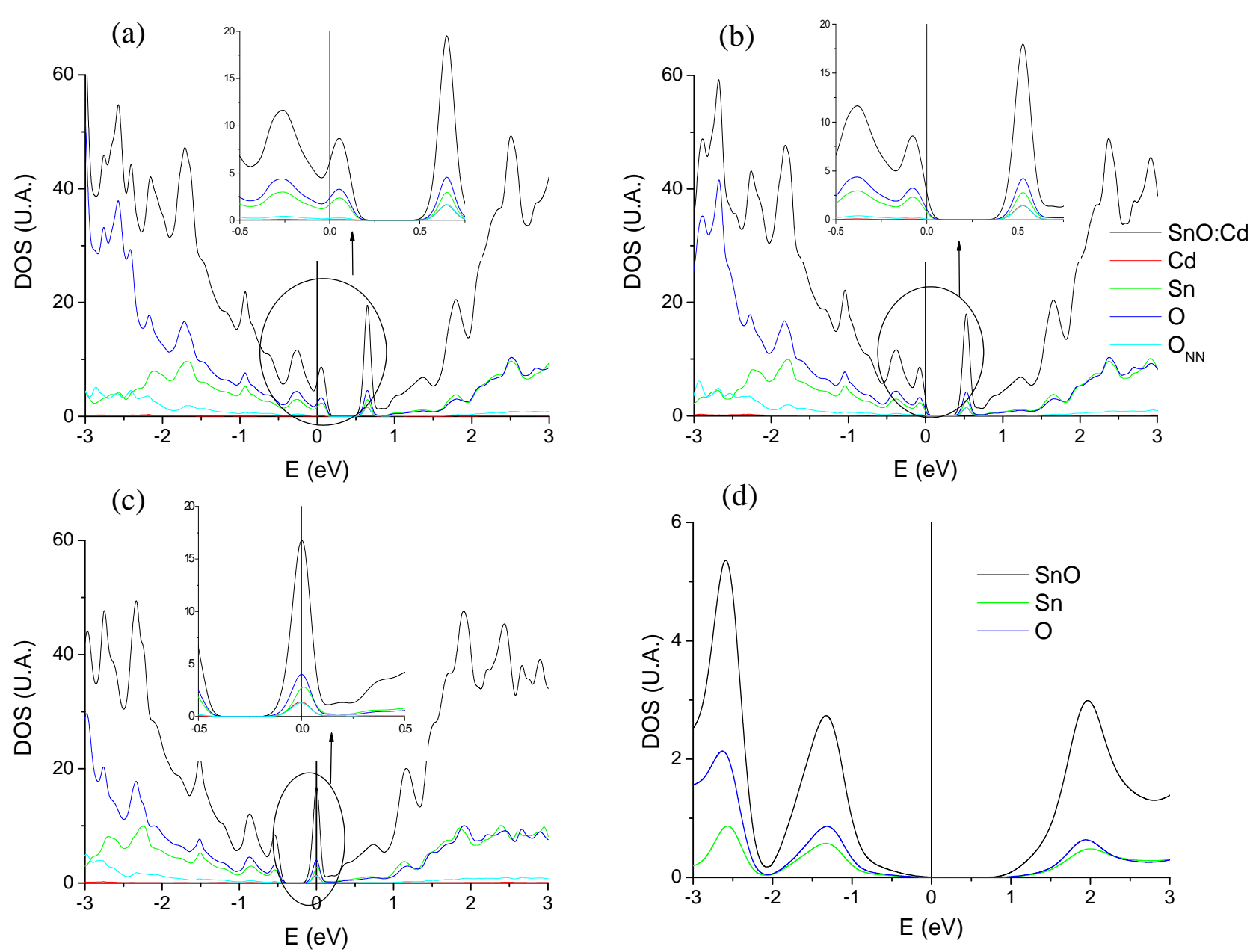

(d)

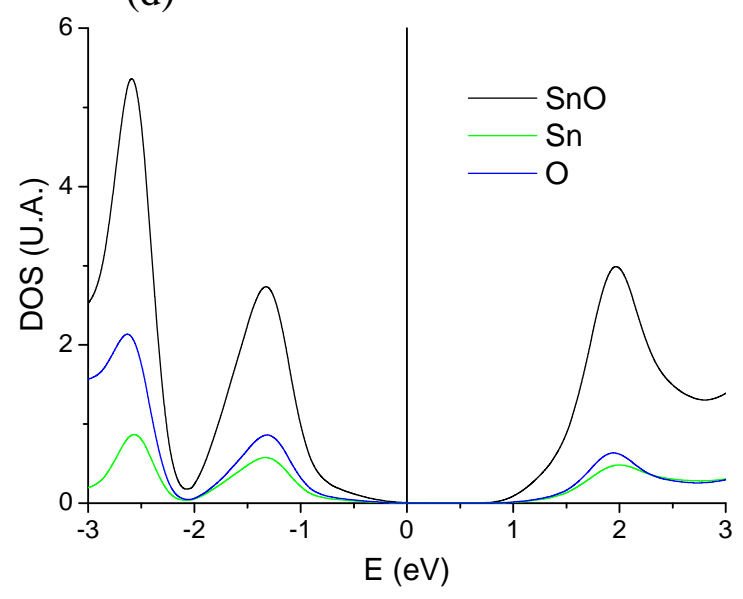

Figura 7.27: Densidades de estados (DOS) del óxido SnO puro y dopado con Cd con las contribuciones de las esferas atómicas de $\mathrm{Sn}, \mathrm{O}, \mathrm{Cd}$ y los vecinos $\mathrm{O}_{\mathrm{NN}}$. Se grafican las DOS correspondientes a los siguientes casos: (a) celda de SnO:Cd descargada, (b) celda de SnO:Cd neutra; (c) celda de SnO:Cd cargada; y (d) SnO puro.

En la Tabla 7.27 se detalla la dependencia del GCE con el estado de carga de la supercelda. Se realizaron cálculos en las aproximaciones CW-GGA y LDA. De la comparación de los parámetros hiperfinos obtenidos con ambas aproximaciones se observa un gran disparidad. Cabe destacar que este sistema ha sido extensamente estudiado por nuestro grupo tanto en el campo teórico [Errico 2007] como en el experimental [Muñoz 2007] y del doble abordaje experimental - ab initio obtuvimos una correcta predicción del GCE con los cálculos realizados con LDA. Se puede observar que en la aproximación LDA al remover un electrón de la celda neutra la magnitud de $\mathrm{V}_{33}$ se ve incrementada en un 50\%, mientras que al agregar un electrón a la celda neutra $\mathrm{V}_{33}$ se ve modificado en signo y magnitud. El parámetro de asimetría es nulo en todos los casos debido a que el método APW+lo mantiene la simetría puntual 
del sitio catiónico de la impureza $\mathrm{Cd}$ en el proceso de relajación de la estructura. Finalmente, la distancia entre el $\mathrm{Cd}$ y los vecinos $\mathrm{O}_{\mathrm{NN}}$ aumenta a medida que se agregan electrones a la celda descargada debido a la repulsión coulombiana.

\begin{tabular}{|c|c|c|c|c|c|}
\hline $\begin{array}{c}\text { Estados de carga } \\
\text { de la celda }\end{array}$ & Aprox. & $\boldsymbol{d}_{N N}(\AA)$ & $\eta$ & $V_{33}\left(10^{21} \mathrm{~V} / \mathrm{m}^{2}\right)$ & Dir. $V_{33}$ \\
\hline \multirow{2}{*}{ Descargada } & LDA & 2.26 & 0.00 & +7.79 & {$\left[\begin{array}{lll}0 & 0 & 1\end{array}\right]$} \\
\hline & CW-GGA & 2.28 & 0.00 & +5.22 & {$\left[\begin{array}{lll}0 & 0 & 1\end{array}\right]$} \\
\hline \multirow{2}{*}{ Neutra } & LDA & 2.27 & 0.00 & +5.26 & {$\left[\begin{array}{lll}0 & 0 & 1\end{array}\right]$} \\
\hline & CW-GGA & 2.31 & 0.00 & +1.93 & {$\left[\begin{array}{lll}0 & 0 & 1\end{array}\right]$} \\
\hline \multirow{2}{*}{ Cargada } & LDA & 2.37 & 0.00 & -4.52 & {$\left[\begin{array}{lll}0 & 0 & 1\end{array}\right]$} \\
\hline & CW-GGA & 2.37 & 0.00 & -4.13 & {$\left[\begin{array}{lll}0 & 0 & 1\end{array}\right]$} \\
\hline
\end{tabular}

Tabla 7.27: Cálculos APW+lo de $d_{N N}, \mathrm{~V}_{33}$ (dirección y magnitud) y $\eta$ en función del estado de carga de la celda de SnO:Cd en las aproximaciones LDA y CW-GGA. Las distancias $d_{N N}$ están dadas en $\AA \quad$ y $V_{33}$ en unidades de $10^{21} \mathrm{~V} / \mathrm{m}^{2}$.

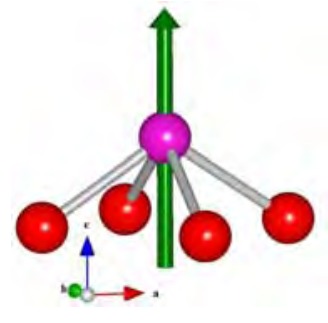

Figura 7.28:

Dirección de $\mathrm{V}_{33}$ en el sitio del Cd en $\mathrm{SnO}$.

En la Tabla 7.28 se detallan las contribuciones $p, d$ y s- $d$ al tensor GCE en función del estado de carga de la celda. Se puede apreciar que la contribución $p$ en general presenta más intensidad que la contribución $d$, mientras que la contribución $s$ - $d$ se vuelve despreciable frente a las otras dos. Además, la simetría $p$ cambian de signo y magnitud al agregar o remover un electrón de la celda neutra, mientras que la simetría $d$ disminuye en magnitud a medida que se agregan electrones a la celda descargada.

En la Figura 7.29 se grafican las densidades PDOS de la impureza Cd en la región del nivel de Fermi. Se observa que en el fondo de la banda de conducción el Cd introduce un nivel de impureza apreciable, el cual presenta una contribución mayoritaria $s$, además se observa una segunda contribución de simetría $p$ y la de simetría $d$ es prácticamente despreciable. El tope de la banda de valencia también presenta las mismas características. Este hecho se corrlaciona con la fuerte dependencia que presentan las componentes $V_{i i}$ de carácter $p$ con el estado de carga de la celda frente a las demás contribuciones (ver Tabla 7.28). 


\begin{tabular}{cccccccccc}
\hline & \multicolumn{3}{c}{ Celda descargada } & \multicolumn{3}{c}{ Celda neutra } & \multicolumn{3}{c}{ Celda cargada } \\
\hline & $\mathbf{V}_{\mathbf{1 1}}$ & $\mathbf{V}_{\mathbf{2 2}}$ & $\mathbf{V}_{\mathbf{3 3}}$ & $\mathbf{V}_{\mathbf{1 1}}$ & $\mathbf{V}_{\mathbf{2 2}}$ & $\mathbf{V}_{\mathbf{3 3}}$ & $\mathbf{V}_{\mathbf{1 1}}$ & $\mathbf{V}_{\mathbf{2 2}}$ & $\mathbf{V}_{\mathbf{3 3}}$ \\
\hline $\boldsymbol{p}$ & -2.58 & -2.58 & +5.17 & -1.46 & -1.46 & +2.92 & +2.85 & +2.85 & -5.70 \\
\hline $\boldsymbol{d}$ & -1.38 & -1.38 & +2.77 & -1.22 & -1.22 & +2.45 & -0.58 & -0.58 & +1.17 \\
\hline $\boldsymbol{s}-\boldsymbol{d}$ & +0.09 & +0.09 & -0.18 & +0.07 & +0.07 & -0.15 & 0.00 & 0.00 & -0.01 \\
\hline total & -3.88 & -3.88 & +7.75 & -2.61 & -2.61 & +5.22 & +2.26 & +2.26 & -4.53 \\
\hline \hline
\end{tabular}

Tabla 7.28: Cálculos FP-APW+lo de las contribuciones $p, d$ y $s$ - $d$ al tensor GCE en función del estado de carga de la celda (aproximación LDA) para Cd en SnO. $V_{i i}(i=1,2,3)$ viene dado en unidades de $10^{21} \mathrm{~V} / \mathrm{m}^{2}$.

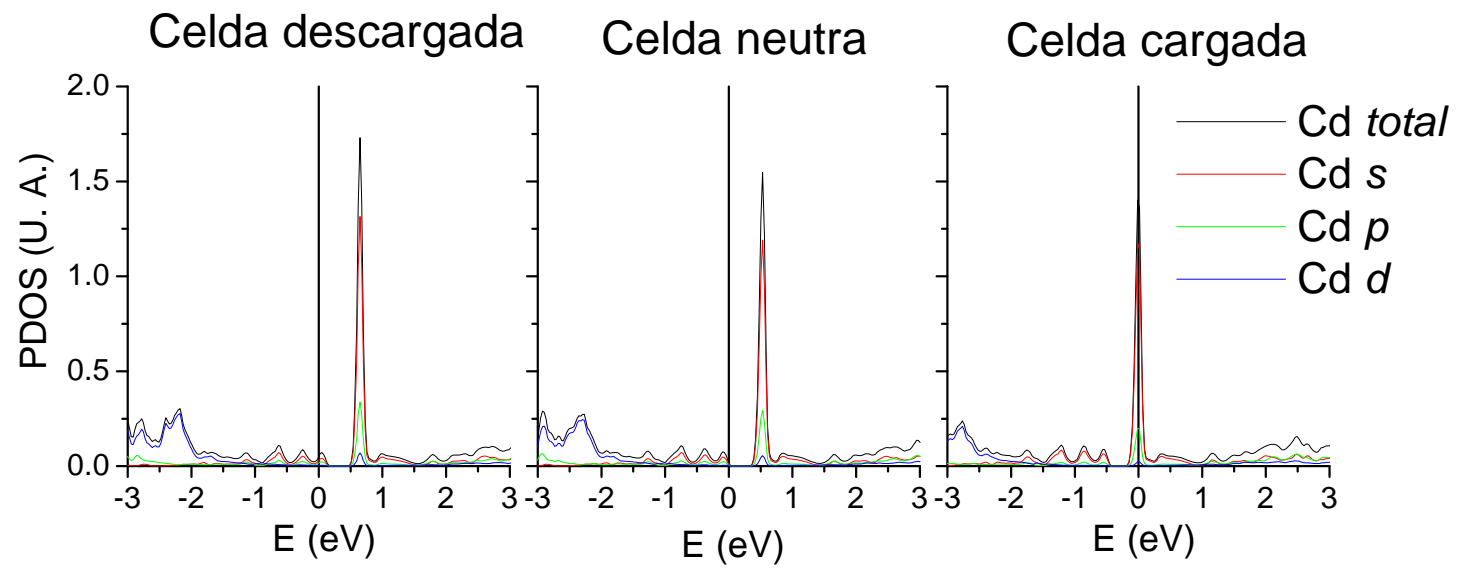

Figura 7.29: PDOS de las simetrías $s, p$ y $d$ de la impureza Cd en el óxido SnO. De izquierda a derecha se muestran las densidades en la región del nivel de Fermi en función del agregado de electrones a la supercelda.

\section{Referencias}

[Marezio 1966] M. Marezio, Acta Cryst. 20, 723 (1966).

[Maslen 1996] N. Maslen, V.A. Streltsov and N. Ishisawa, Acta Cryst. B 52, 414 (1996).

[Norrestam 1968] R. Norrestam, Ark. Kemi. 29, 343 (1968).

[Kisi 1989] E.H. Kisi and M.M. Elcombe, Acta Cryst. C 45, 1867 (1989). 
[Bolzan 1997] A. Bolzan, C. Frong, B. Kennedy and C. Howard, Acta Cryst. B 53, 373 (1997).

[Wang 2004] X. Wang, F.X. Zhang, I Loa, K. Syassen, M. Hanfland, Y.L. Mathins, Phys. Status Solidi B 241, 3168 (2004).

[Muñoz 2009] E.L. Muñoz, D. Richard, L.A. Errico, M. Rentería, Physica B 404, 2757 (2009).

[Muñoz 2011] E.L. Muñoz, D. Richard, P.D. Eversheim and M. Rentería, Hyperfine Interact. 197, 181 (2011).

[Errico 2007] L.A. Errico, M. Rentería and H.M. Petrilli, Phys. Rev. B 75, 155209 (2007).

[Muñoz 2007] E.L. Muñoz, A.W. Carbonari, L.A. Errico, A.G. Bibiloni, H. M. Petrilli, M. Rentería, Hyp. Int. 178, 37 (2007). 


\section{Capítulo $\mathbf{N}^{\mathbf{0}} \mathbf{8}$}

\section{Comparación de resultados y discusión}

\section{8-1 Comparación de resultados PAC con predicciones APW+lo en los óxidos con estructura Bixbita}

En este Capítulo se comparan los resultados experimentales del tensor GCE obtenidos con la técnica PAC y los cálculos ab initio realizados con el método APW+lo en los sistemas abordados en la presente Tesis. En cada caso se determina el estado de carga de la celda vía la mejor comparación del GCE como así también cuál es la aproximación (GGA, LDA o CW-GGA) de las utilizadas en el presente trabajo que mejor predice los parámetros hiperfinos observados en el sitio de la impureza Cd. Cabe destacar que los parámetros hiperfinos experimentales que se muestran en cada tabla corresponden al GCE del estado final estable alcanzado por la sonda ${ }^{111} \mathrm{Cd}$ luego del proceso dinámico experimentado a una temperatura dada.

Primero se detallan los resultados obtenidos en los óxidos con estructura bixbita $\left(\mathrm{In}_{2} \mathrm{O}_{3}, \mathrm{Sc}_{2} \mathrm{O}_{3}\right.$ y $\left.\mathrm{Y}_{2} \mathrm{O}_{3}\right)$ debido a que presentan propiedades estructurales y electrónicas similares a altas temperatura, de hecho coinciden los estados de carga asignados en dicho rango.

\section{8-1-1 Comparación de resultados en $\operatorname{In}_{2} \mathrm{O}_{3}:{ }^{111} \mathrm{Cd}$}

En este apartado se comparan resultados PAC y cálculos ab initio del tensor GCE observado por la sonda-impureza ${ }^{111} \mathrm{Cd}$ en los sitios catiónicos de la bixbita $\operatorname{In}_{2} \mathrm{O}_{3}$. Además, se comparan los resultados experimentales con cálculos del GCE realizados con el modelo PCM. En la Tabla 8.1 se muestran los resultados PAC, los cálculos PCM y las predicciones APW+lo en función del estado de carga de la celda. Se puede observar que los resultados experimentales medidos a $873 \mathrm{~K}$ para el caso de ${ }^{111} \mathrm{Cd}$ en sitio $C$ se encuentran en excelente acuerdo con los cálculos realizados con celda cargada en la aproximación LDA y en muy buen acuerdo con la aproximación CW-GGA. Para los resultados PAC obtenidos a temperatura ambiente para ${ }^{111} \mathrm{Cd}$ en sitio $\mathrm{C}$ se observa un buen acuerdo con el estado de carga cargado en ambas aproximaciones. Para Cd en sitio D se aprecia que no es posible inferir el estado de carga de la impureza en este sitio 
debido a que el GCE calculado en dicho sitio no presenta dependencia con el estado de carga de la celda. Finalmente, comparando la dependencia de los parámetros hiperfinos en función de la temperatura (ver Figura 5.6) con el estudio APW+lo del GCE en función del estado de carga se aprecia que el estado final del Cd en sitio C corresponde a la celda cargada para todo el rango de temperatura, mientras que para Cd en sitio D si bien no se puede establecer el estado de carga, es de esperar que tenga el mismo estado que el sitio $\mathrm{C}$ debido a que los estados aceptores en el gap del semiconductor son similares en energía. Finalmente, las fracciones ajustadas en el experimento están en excelente acuerdo con la relación cristalográfica de los sitios catiónicos $\left(f_{C}: f_{D}=3: 1\right)$.

Desde la comparación entre los resultados experimentales y las predicciones PCM se observa que para el caso del Cd en el sitio D existe un excelente acuerdo entre las predicciones PCM con celda no relajada y los resultados PAC. Sin embargo, debido al hecho que la impureza Cd introduce relajaciones estructurales en la red del semiconductor, hemos realizado cálculos PCM con las posiciones relajadas obtenidas con el método FP-APW+lo para el caso de celda cargada (ver Apéndice III). Desde la comparación con los cálculos PCM para celda relajada se aprecia una discrepancia con la componente principal $\mathrm{V}_{33}$ del $10 \%$. Para el caso del Cd en el sitio C, se observa que las predicciones PCM con celda no relajada de $\mathrm{V}_{33}$ difiere en más del 20\% con los valores experimentales, mientras que el parámetro de asimetría presenta discrepancias entre el 10 y el 20\% para ambas temperaturas. Considerando las relajaciones predichas por el método ab initio (ver Apéndice III), se observa que la componente $\mathrm{V}_{33}$ difiere en un $20 \%$ respecto a los valores experimentales, mientras que el parámetro $\eta$ presenta discrepancias entre el 10 y el 30\%. Por lo tanto, el modelo PCM resulta obsoleto a la hora de describir las interacciones hiperfinas observadas por la impureza Cd en el $\operatorname{In}_{2} \mathrm{O}_{3}$. 


\begin{tabular}{|c|c|c|c|c|c|c|c|c|c|}
\hline \multirow{2}{*}{$\begin{array}{c}\text { Estado de } \\
\text { carga de la } \\
\text { celda } \\
\end{array}$} & \multirow{2}{*}{ Aprox. } & \multicolumn{4}{|c|}{ Sitio D } & \multicolumn{4}{|c|}{ Sitio C } \\
\hline & & $\boldsymbol{d}_{N N}$ & $V_{33}$ & $\eta$ & Dir. $V_{33}$ & $\boldsymbol{d}_{N N}$ & $V_{33}$ & $\eta$ & Dir. $V_{33}$ \\
\hline Descargada & CW-GGA & 2.20 & +6.64 & 0.00 & {$\left[\begin{array}{lll}1 & 1 & 1\end{array}\right]$} & $\begin{array}{l}2.11 \\
2.23 \\
2.30 \\
\end{array}$ & 2.77 & 0.58 & {$\left[\begin{array}{lll}0 & 0 & 1\end{array}\right]$} \\
\hline \multirow{2}{*}{ Neutra } & LDA & 2.23 & +6.93 & 0.00 & {$\left[\begin{array}{lll}1 & 1 & 1\end{array}\right]$} & $\begin{array}{l}2.14 \\
2.25 \\
2.31\end{array}$ & -3.13 & 0.08 & {$\left[\begin{array}{lll}1 & -0.6 & 0\end{array}\right]$} \\
\hline & CW-GGA & 2.23 & +7.15 & 0.00 & {$\left[\begin{array}{lll}1 & 1 & 1\end{array}\right]$} & $\begin{array}{l}2.14 \\
2.27 \\
2.30 \\
\end{array}$ & -3.08 & 0.01 & {$\left[\begin{array}{lll}1 & -0.6 & 0\end{array}\right]$} \\
\hline \multirow{2}{*}{ Cargada } & LDA & 2.27 & +7.29 & 0.00 & {$\left[\begin{array}{lll}1 & 1 & 1\end{array}\right]$} & $\begin{array}{l}2.20 \\
2.30 \\
2.31\end{array}$ & +6.16 & 0.69 & {$\left[\begin{array}{lll}0.8 & 1 & 0\end{array}\right]$} \\
\hline & CW-GGA & 2.28 & +7.68 & 0.00 & {$\left[\begin{array}{lll}1 & 1 & 1\end{array}\right]$} & $\begin{array}{l}2.20 \\
2.30 \\
2.32 \\
\end{array}$ & +5.72 & 0.72 & {$\left[\begin{array}{lll}0.8 & 1 & 0\end{array}\right]$} \\
\hline Modelo & Celda & $\boldsymbol{d}_{N N}$ & $V_{33}$ & $\eta$ & Dir. $V_{33}$ & $d_{N N}$ & $V_{33}$ & $\eta$ & Dir. $V_{33}$ \\
\hline PCM & $\begin{array}{l}\text { No } \\
\text { relajada }\end{array}$ & 2.19 & +7.54 & 0.00 & {$\left[\begin{array}{lll}1 & 1 & 1\end{array}\right]$} & $\begin{array}{l}2.12 \\
2.19 \\
2.21 \\
\end{array}$ & -4.87 & 0.82 & {$\left[\begin{array}{lll}1 & -0.8 & 0\end{array}\right]$} \\
\hline PCM & Relajada & 2.28 & +6.95 & 0.00 & {$\left[\begin{array}{lll}1 & 1 & 1\end{array}\right]$} & $\begin{array}{l}2.20 \\
2.30 \\
2.32 \\
\end{array}$ & +5.11 & 0.63 & {$\left[\begin{array}{lll}0.7 & 1 & 0\end{array}\right]$} \\
\hline Método & $T(K)$ & $f(\%)$ & $V_{33}$ & $\eta$ & Dir. $V_{33}$ & $f(\%)$ & $V_{33}$ & $\eta$ & Dir. $V_{33}$ \\
\hline PAC & $\mathrm{T}=298 \mathrm{~K}$ & $28.5(4.0)$ & 7.57(11) & 0.00 & - & $71.5(4.0)$ & $5.83(44)$ & $0.94(4)$ & - \\
\hline PAC & $\mathrm{T}=873 \mathrm{~K}$ & $22.6(2.0)$ & $7.58(1)$ & 0.00 & - & $77.4(2.0)$ & $6.14(2)$ & $0.70(1)$ & - \\
\hline
\end{tabular}

Tabla 8.1: Resultados PAC, PCM utilizando las posiciones atómicas de la celda relajada y no relajada y de cálculos $a b$ initio del GCE observado por la impureza Cd en los sitios C y D del $\operatorname{In}_{2} \mathrm{O}_{3}$. Las predicciones APW+lo de $d_{N N}, V_{33}$ (dirección y magnitud) y $\eta$ se detallan en función del estado de carga de la celda en las aproximaciones LDA y CW-GGA. Las distancias $d_{N N}$ están dadas en $\AA$ y $\mathrm{V}_{33}$ en unidades de $10^{21} \mathrm{~V} / \mathrm{m}^{2}$.

Teniendo en cuenta que la celda cargada corresponde a agregar un electrón a la celda de $\mathrm{In}_{2} \mathrm{O}_{3}$ y que los estados electrónicos de ésta que aportan al nivel de impureza se localizan mayoritariamente en el entorno del Cd y sus vecinos $\mathrm{O}_{\mathrm{NN}}$ (tal como se verá en la sección 8-5-1), se concluye que el estado final estable del Cd en el sitio C no presenta huecos electrónicos en su entorno en todo el rango de temperatura, mientras que para Cd en sitio D si bien no es posible determinar el estado de carga de la impureza, es de 
esperar que tenga el mismo estado de carga que el sitio C por la similitud en energía de los estados de impureza y en la localización espacial de estos estados electrónicos.

\section{8-1-2 Comparación de resultados en $\mathrm{Sc}_{2} \mathrm{O}_{3}:{ }^{111} \mathrm{Cd}$}

En esta sección se aborda el sesquióxido $\mathrm{Sc}_{2} \mathrm{O}_{3}$ dopado con ${ }^{111} \mathrm{Cd}$. En la Tabla 8.2 se detallan los parámetros hiperfinos experimentales, las predicciones PCM y los resultados de los cálculos ab initio que caracterizan el tensor GCE observado por la sonda-impureza ${ }^{111} \mathrm{Cd}$ en los sitios catiónicos $\mathrm{C}$ y $\mathrm{D}$ de la bixbita $\mathrm{Sc}_{2} \mathrm{O}_{3}$. Cabe mencionar que las medidas experimentales tabuladas corresponden a los rangos de temperatura donde las interacciones se mantenían constantes. En la Figura 5.8 del Capítulo 5 se observa que en la representación de $V_{33}$ y $\eta$ vs. $T$ se obtienen cuatro rangos de temperatura donde dichos parámetros se mantienen aproximadamente constantes.

Se puede apreciar en la Tabla 8.2 que para el caso de ${ }^{111} \mathrm{Cd}$ en sitio C existe un excelente acuerdo entre las medidas PAC realizadas a $900 \mathrm{~K}$ y los cálculos APW+lo en la aproximación CW-GGA realizados con la celda cargada, mientras que se observa un muy buen acuerdo con los cálculos realizados con LDA y GGA también con celda cargada. Además, las medidas de la componente principal $V_{33}$ realizadas a $400 \mathrm{~K}$ en el sitio C están en muy buen acuerdo con los cálculos correspondientes a celda neutra en la aproximación LDA y presenta un buen acuerdo con las aproximaciones CW-GGA y GGA, mientras que las medidas del parámetro de asimetría presentan discrepancias entre 30\% (CW-GGA) y 50 \% (LDA). Cabe destacar que los $\eta$ más bajos calculados corresponden a celda neutra. En cuanto a las medidas tomadas a $295 \mathrm{~K}$ en el sitio C, se aprecia un excelente acuerdo entre los parámetros experimentales y los cálculos APW+lo correspondientes a celda descargada. Finalmente, las medidas realizadas a 10 $\mathrm{K}$ en el sitio $\mathrm{C}$ presentan un excelente acuerdo con los cálculos de la celda cargada realizados en la aproximación CW-GGA, mientras que el acuerdo es muy bueno para el mismo estado de carga en las aproximaciones LDA y GGA.

En el caso de ${ }^{111} \mathrm{Cd}$ en el sitio D, no es posible asignarle estado de carga alguno a las medidas PAC puesto que el GCE calculado en dicho sitio no presenta dependencia con el estado de carga de la celda. Sin embargo, se espera que tenga el mismo estado que la impureza localizada en el sitio C debido a que los estados aceptores son similares en energía. 


\begin{tabular}{|c|c|c|c|c|c|c|c|c|c|}
\hline \multirow{2}{*}{$\begin{array}{c}\text { Estado de } \\
\text { carga de la } \\
\text { celda }\end{array}$} & \multirow{2}{*}{ Aprox. } & \multicolumn{4}{|c|}{ Sitio D } & \multicolumn{4}{|c|}{ Sitio C } \\
\hline & & $d_{N N}$ & $V_{33}$ & $\eta$ & Dir. $V_{33}$ & $d_{N N}$ & $V_{33}$ & $\eta$ & Dir. $V_{33}$ \\
\hline Descargada & CW-GGA & 2.22 & +8.24 & 0.00 & {$\left[\begin{array}{lll}1 & 1 & 1\end{array}\right]$} & $\begin{array}{l}2.10 \\
2.24 \\
2.32 \\
\end{array}$ & +3.06 & 0.64 & {$\left[\begin{array}{lll}0 & 0 & 1\end{array}\right]$} \\
\hline \multirow{3}{*}{ Neutra } & LDA & 2.25 & +8.17 & 0.00 & {$\left[\begin{array}{lll}1 & 1 & 1\end{array}\right]$} & $\begin{array}{l}2.13 \\
2.25 \\
2.33\end{array}$ & -4.40 & 0.53 & {$\left[\begin{array}{lll}1 & -0.7 & 0\end{array}\right]$} \\
\hline & CW-GGA & 2.25 & +8.32 & 0.00 & {$\left[\begin{array}{lll}1 & 1 & 1\end{array}\right]$} & $\begin{array}{l}2.14 \\
2.28 \\
2.32 \\
\end{array}$ & -3.81 & 0.34 & {$\left[\begin{array}{lll}1 & -0.7 & 0.7\end{array}\right]$} \\
\hline & GGA & 2.25 & +8.25 & 0.00 & {$\left[\begin{array}{lll}1 & 1 & 1\end{array}\right]$} & $\begin{array}{l}2.14 \\
2.28 \\
2.32 \\
\end{array}$ & -3.91 & 0.40 & {$\left[\begin{array}{lll}1 & -0.7 & 0 .\end{array}\right]$} \\
\hline \multirow{3}{*}{ Cargada } & LDA & 2.28 & +8.05 & 0.00 & {$\left[\begin{array}{lll}1 & 1 & 1\end{array}\right]$} & $\begin{array}{l}2.17 \\
2.30 \\
2.31 \\
\end{array}$ & +7.37 & 0.67 & {$\left[\begin{array}{lll}0.8 & 1 & 0\end{array}\right]$} \\
\hline & CW-GGA & 2.28 & +8.16 & 0.00 & {$\left[\begin{array}{lll}1 & 1 & 1\end{array}\right]$} & $\begin{array}{l}2.19 \\
2.31 \\
2.32\end{array}$ & +6.50 & 0.71 & {$\left[\begin{array}{lll}0.8 & 1 & 0\end{array}\right]$} \\
\hline & GGA & 2.28 & +8.18 & 0.00 & {$\left[\begin{array}{lll}1 & 1 & 1\end{array}\right]$} & $\begin{array}{l}2.19 \\
2.32 \\
2.32\end{array}$ & +6.99 & 0.66 & {$\left[\begin{array}{lll}0.8 & 1 & 0\end{array}\right]$} \\
\hline Modelo & Celda & $\boldsymbol{d}_{N N}$ & $V_{33}$ & $\eta$ & Dir. $V_{33}$ & $\boldsymbol{d}_{N N}$ & $V_{33}$ & $\eta$ & Dir. $V_{33}$ \\
\hline PCM & No relajada & 2.12 & +7.05 & 0.00 & {$\left[\begin{array}{lll}1 & 1 & 1\end{array}\right]$} & $\begin{array}{l}2.08 \\
2.12 \\
2.16 \\
\end{array}$ & -3.81 & 0.95 & {$\left[\begin{array}{lll}1 & -0.6 & 0\end{array}\right]$} \\
\hline PCM & Relajada & 2.28 & +6.82 & 0.00 & {$\left[\begin{array}{lll}1 & 1 & 1\end{array}\right]$} & $\begin{array}{l}2.19 \\
2.31 \\
2.32 \\
\end{array}$ & +5.28 & 0.57 & {$\left[\begin{array}{lll}0.9 & 1 & 0\end{array}\right]$} \\
\hline Método & $T(K)$ & $f(\%)$ & $V_{33}$ & $\eta$ & Dir. $V_{33}$ & $f$ & $V_{33}$ & $\eta$ & Dir. $V_{33}$ \\
\hline PAC & 10 & $27(4)$ & $8.52(15)$ & 0.00 & - & $73(4)$ & $6.50(4)$ & $0.73(1)$ & - \\
\hline PAC & 295 & $25(4)$ & 9.31(3) & 0.00 & - & $75(4)$ & $3.02(6)$ & $0.72(2)$ & - \\
\hline PAC & 400 & $15(2)$ & $8.91(26)$ & 0.00 & - & $85(2)$ & $4.67(33)$ & 0.00 & - \\
\hline PAC & 900 & 25 & $8.23(1)$ & 0.00 & - & 75 & $6.57(2)$ & $0.70(1)$ & - \\
\hline
\end{tabular}

Tabla 8.2: Resultados PAC, PCM utilizando las posiciones atómicas de la celda relajada y no relajada y cálculos $a b$ initio del GCE observado por la impureza $\mathrm{Cd}$ en los sitios $\mathrm{C}$ y D del $\mathrm{Sc}_{2} \mathrm{O}_{3}$. Las predicciones APW+lo de $d_{N N}, \mathrm{~V}_{33}$ (dirección y magnitud) y $\eta$ se detallan en función del estado de carga de la celda en las aproximaciones LDA y CW-GGA. Las distancias $d_{N N}$ están dadas en $\AA$ y $V_{33}$ en unidades de $10^{21} \mathrm{~V} / \mathrm{m}^{2}$. 
Por lo tanto, debido a que el estado de carga predicho a altas temperaturas es cargado y teniendo en cuenta que los estados electrónicos de éste estado que contribuyen al nivel de impureza del sistema $\mathrm{Sc}_{2} \mathrm{O}_{3}:{ }^{111} \mathrm{Cd}$ se concentran mayoritariamente en las ligaduras del Cd y sus vecinos $\mathrm{O}_{\mathrm{NN}}$ (ver sección 8-5-3), entonces a altas temperaturas la sonda-impureza Cd no presenta huecos electrónicos en su entorno. En el rango de temperatura 350 - $450 \mathrm{~K}$ (ver figura 5.8) el ${ }^{111} \mathrm{Cd}$ presentaría un hueco electrónico en su vecindad (celda neutra). Entre 200 y $300 \mathrm{~K}$ el Cd presentaría dos huecos electrónicos (celda descargada), mientras que a muy bajas temperaturas (10 - $150 \mathrm{~K}$ ) el entorno de la sonda Cd volvería a recuperar electrones quedando sin huecos (celda cargada) [Muñoz 2011]. Cabe destacar que para validar las asignaciones realizadas en el rango de temperaturas donde la atenuación dinámica es apreciable es necesario calcular la dependencia del GCE con el estado de carga con mayor resolución (descargando la celda en fracciones de $0.1 e^{-}$, por ejemplo) y removiendo más carga de la celda.

Desde la comparación entre los resultados experimentales y las predicciones PCM para el caso del Cd en el sitio D en celda no relajada se observa que la componente principal $\mathrm{V}_{33}$ presenta discrepancias del 10 y el 20\% respecto de los resultados PAC, mientras que al considerar las relajaciones FP-APW+lo (ver Apéndice III) estas discrepancias se ven aumentadas en más del 3\%. En el caso de la impureza Cd en el sitio $\mathrm{C}$ para celda no relajada, la componente $\mathrm{V}_{33}$ presenta discrepancias que van desde el 25\% hasta el 40\% para las medidas PAC tabuladas y el parámetro de asimetría difiere en más del 25\%, mientras que si se consideran las relajaciones predichas por el método FP-APW+lo $\mathrm{V}_{33}$ discrepa en más del $20 \%$ y $\eta$ en más del 15\%. Entonces, al igual que en el caso de $\mathrm{In}_{2} \mathrm{O}_{3}$, el modelo PCM resulta obsoleto para describir las interacciones hiperfinas observadas por la impureza Cd en la red huésped del $\mathrm{Sc}_{2} \mathrm{O}_{3}$.

\section{8-1-3 Comparación de resultados en $\mathrm{Y}_{2} \mathrm{O}_{3}:{ }^{111} \mathrm{Cd}$}

En este apartado se comparan los resultados PAC, las predicciones PCM y los resultados de los cálculos $a b$ initio en el sistema $\mathrm{Y}_{2} \mathrm{O}_{3}:{ }^{111} \mathrm{Cd}$. En la Tabla 8.3 se describen los parámetros hiperfinos que caracterizan el tensor GCE en los sitios C y D donde se aloja la impureza ${ }^{111} \mathrm{Cd}$. 


\begin{tabular}{|c|c|c|c|c|c|c|c|c|c|}
\hline \multirow{2}{*}{$\begin{array}{c}\text { Estado de } \\
\text { carga de la } \\
\text { celda }\end{array}$} & \multirow{2}{*}{ Aprox. } & \multicolumn{4}{|c|}{ Sitio D } & \multicolumn{4}{|c|}{ Sitio C } \\
\hline & & $\boldsymbol{d}_{N N}$ & $V_{33}$ & $\eta$ & Dir. $V_{33}$ & $d_{N N}$ & $V_{33}$ & $\eta$ & Dir. $V_{33}$ \\
\hline Descargada & CW-GGA & 2.28 & +7.15 & 0.00 & {$\left[\begin{array}{lll}1 & 1 & 1\end{array}\right]$} & $\begin{array}{l}2.19 \\
2.33 \\
2.35 \\
\end{array}$ & -2.05 & 0.54 & {$\left[\begin{array}{lll}-0.4 & 1 & 0\end{array}\right]$} \\
\hline \multirow{2}{*}{ Neutra } & LDA & 2.31 & +7.10 & 0.00 & {$\left[\begin{array}{lll}1 & 1 & 1\end{array}\right]$} & $\begin{array}{l}2.20 \\
2.36 \\
2.37\end{array}$ & -3.00 & 0.27 & {$\left[\begin{array}{lll}-0.6 & 1 & 0\end{array}\right]$} \\
\hline & CW-GGA & 2.32 & +7.25 & 0.00 & {$\left[\begin{array}{lll}1 & 1 & 1\end{array}\right]$} & $\begin{array}{l}2.22 \\
2.36 \\
2.38 \\
\end{array}$ & -2.58 & 0.05 & {$\left[\begin{array}{lll}-0.5 & 1 & 0\end{array}\right]$} \\
\hline \multirow{2}{*}{ Cargada } & LDA & 2.34 & +7.21 & 0.00 & {$\left[\begin{array}{lll}1 & 1 & 1\end{array}\right]$} & $\begin{array}{l}2.26 \\
2.36 \\
2.42 \\
\end{array}$ & +4.06 & 0.86 & {$\left[\begin{array}{lll}1 & 0.8 & 0\end{array}\right]$} \\
\hline & CW-GGA & 2.36 & +7.28 & 0.00 & {$\left[\begin{array}{lll}1 & 1 & 1\end{array}\right]$} & $\begin{array}{l}2.27 \\
2.35 \\
2.45 \\
\end{array}$ & +3.73 & 0.88 & {$\left[\begin{array}{lll}1 & 0.7 & 0\end{array}\right]$} \\
\hline Modelo & Celda & $\boldsymbol{d}_{N N}$ & $V_{33}$ & $\eta$ & Dir. $V_{33}$ & $d_{N N}$ & $V_{33}$ & $\eta$ & Dir. $V_{33}$ \\
\hline PCM & $\begin{array}{c}\text { No } \\
\text { relajada }\end{array}$ & 2.28 & +7.75 & 0.00 & {$\left[\begin{array}{lll}1 & 1 & 1\end{array}\right]$} & $\begin{array}{l}2.24 \\
2.27 \\
2.33 \\
\end{array}$ & -3.51 & 0.63 & {$\left[\begin{array}{lll}-0.6 & 1 & 0\end{array}\right]$} \\
\hline PCM & Relajada & 2.36 & +6.95 & 0.00 & {$\left[\begin{array}{lll}1 & 1 & 1\end{array}\right]$} & $\begin{array}{l}2.27 \\
2.35 \\
2.45 \\
\end{array}$ & +3.97 & 0.67 & {$\left[\begin{array}{lll}1 & 0.6 & 0\end{array}\right]$} \\
\hline Método & $T(K)$ & $f(\%)$ & $V_{33}$ & $\eta$ & Dir. $V_{33}$ & $f$ & $V_{33}$ & $\eta$ & Dir. $V_{33}$ \\
\hline PAC & 295 & 25 & $7.39(28)$ & 0.00 & - & 75 & $5.27(24)$ & $0.49(4)$ & - \\
\hline PAC & 500 & 25 & $7.29(6)$ & 0.00 & - & 75 & $5.35(18)$ & 0.00 & - \\
\hline PAC & 900 & 25 & $7.42(1)$ & 0.00 & - & 75 & $4.41(3)$ & $0.76(1)$ & - \\
\hline
\end{tabular}

Tabla 8.3: Resultados PAC, predicciones PCM utilizando las posiciones atómicas de la celda relajada y no relajada y resultados de cálculos $a b$ initio del GCE observado por la impureza ${ }^{111} \mathrm{Cd}$ en los sitios $\mathrm{C}$ y D del $\mathrm{Y}_{2} \mathrm{O}_{3}$. Las predicciones APW+lo de $d_{N N}, \mathrm{~V}_{33}$ (dirección y magnitud) y $\eta$ se detallan en función del estado de carga de la celda en las aproximaciones LDA y CW-GGA. Las distancias $d_{N N}$ están dadas en $\AA$ y $\mathrm{V}_{33}$ en unidades de $10^{21} \mathrm{~V} / \mathrm{m}^{2}$.

Se puede apreciar que los parámetros hiperfinos medidos que caracterizan al ${ }^{111} \mathrm{Cd}$ en el sitio C a $900 \mathrm{~K}$ están en muy buen acuerdo con los cálculos APW+lo correspondientes a la celda cargada en ambas aproximaciones (LDA y CW-GGA). En el caso de las medidas realizadas a $500 \mathrm{~K}$ se aprecia un excelente acuerdo entre el 
parámetro de asimetría PAC y el calculado en celda neutra con la aproximación CWGGA pero la componente principal experimental presenta discrepancias del orden del $50 \%$ para el caso de celda neutra. Finalmente, el parámetro de asimetría medido a $295 \mathrm{~K}$ está en muy buen acuerdo con el cálculo realizado con celda descargada, sin embargo la medida de $\mathrm{V}_{33}$ a esta temperatura difiere en más del 100\% del cálculo de $\mathrm{V}_{33}$ con celda descargada. Cabe destacar que los espectros ajustados a temperaturas intermedias, esto es donde la atenuación dinámica es muy importante, no están bien definidos.

En cuanto a las medidas PAC que caracterizan a la sonda ${ }^{111} \mathrm{Cd}$ localizada en el sitio D, se aprecia que están en excelente acuerdo con los cálculos realizados (con las aproximaciones CW-GGA y LDA) con los tres estados de carga por lo que no es posible asignarle estado de carga alguno. Sin embargo, es de esperar que presente el mismo estado de carga que el sitio $\mathrm{C}$ debido a la similitud energía que presentan los estados aceptores introducidos en el gap del semiconductor.

Por lo tanto, a partir del excelente acuerdo encontrado entre los resultados PAC a altas temperaturas (donde la interacción hiperfina se hace estática) y los cálculos FPAPW+lo correspondientes a celda cargada y teniendo en cuenta que el electrón agregado a la celda neutra se localiza mayoritariamente en el entorno de la impureza Cd y sus primeros vecinos oxígeno (tal como se verá en la siguiente sección), se concluye que el estado de carga de la impureza a altas temperaturas es cargado. Por otro lado, a partir de las discrepancias encontradas entre las medidas de $V_{33}$ realizadas a 295 y 500 $\mathrm{K}$ con los cálculos APW+lo en función del estado de carga, se concluye que es necesario realizar cálculos variando la carga de la supercelda con mayor resolución y removiendo más electrones de la celda para caracterizar correctamente cada estado final estable alcanzado en el rango de temperatura donde la atenuación dinámica es importante.

Desde la comparación entre los resultados PAC y las prediccciones PCM para el caso del Cd en el sitio D con celda no relajada se aprecia que existe un acuerdo aceptable. Si se consideran las relajaciones estructurales predichas por el método FPAPW+lo (ver Apéndice III), se aprecia nuevamente un buen acuerdo entre los resultados experimentales y las predicciones PCM con celda relajada. A partir de la comparación entre las medidas PAC y los cálculos PCM para el Cd en el sitio C con celda no relajada se aprecian discrepancias en $\mathrm{V}_{33}$ mayores al 30\% para las medidas a temperaturas intermedias y mayores al $20 \%$ para altas temperaturas, mientras que para $\eta$ las discrepancias son del orden del 15\% respecto a las medidas PAC. Para el caso de 
la impureza $\mathrm{Cd}$ en el sitio $\mathrm{C}$ con celda relajada, se aprecia que $\mathrm{V}_{33} \mathrm{y} \eta$ difieren en un $10 \%$ respecto a las medidas a altas temperaturas, mientras que para el caso de temperaturas intermedias $V_{33}$ difiere en un $30 \%$, y $\eta$ en un $15 \%$ con la medida a $295 \mathrm{~K}$ y en un $70 \%$ respecto al valor experimental a $500 \mathrm{~K}$. Si bien en el sitio D se observa un buen acuerdo entre las resultados PAC y las predicciones PCM, en el caso del Cd en el sitio C se aprecia que PCM resulta obsoleto para describir las interacciones observadas por la impureza en el sitio asimétrico C. Cabe destacar que las propiedades de impureza del Cd en la red huésped se hacen más evidentes en el sitio más asimétrico (sitio C) por lo que se concluye que el acuerdo observado en el sitio D es meramente fortuito.

\section{8-2 Comparación de resultados PAC con cálculos APW+lo en $\mathrm{SnO}_{2}:{ }^{111} \mathrm{Cd}$}

En esta sección se comparan los resultados experimentales y los cálculos ab initio realizados en el óxido $\mathrm{SnO}_{2}$ dopado con sondas-impurezas de ${ }^{111} \mathrm{Cd}$. En la Tabla 8.4 se detallan los parámetros hiperfinos teóricos y experimentales que caracterizan el tensor GCE observado por el Cd en el sitio catiónico de la estructura rutilo. En la Tabla se puede apreciar que la interacción HFI1 mayoritaria a bajas y altas temperaturas (ver figura 5.12 del Capítulo 5) se corresponde con el cálculo del GCE realizado con celda cargada, los resultados APW+lo (en las tres aproximaciones) se encuentran en excelente acuerdo con los parámetros PAC de dicha interacción.

En base a lo discutido en la sección 7-2-5 del Capítulo 7 y tal como se verá en la sección 8-5-4, donde se deduce que los estados electrónicos de la celda cargada que contribuyen al nivel de impureza se encuentran mayoritariamente en las esferas del Cd y los $\mathrm{O}_{\mathrm{NN}}$, se puede afirmar que la interacción HFI1 corresponde a la impureza ${ }^{111} \mathrm{Cd}$ doblemente ionizada y localizada en el sitio de catión.

En cuanto a la segunda interacción, se observa en la figura 5.12 que entre 450 y 900 K la población de HFI2 se equilibra con HFI1 variando la población de manera aleatoria alrededor del 50\%, mientras que entre 300 y 450 K HFI2 se hace mayoritaria. Teniendo en cuenta que la componente $\mathrm{V}_{33}$ de HFI2 oscila en todo el rango de temperaturas aproximadamente entre 6 y $7.5 \times 10^{21} \mathrm{~V} / \mathrm{m}^{2}$ y que el parámetro $\eta$ de HFI2 varía entre 0.2 y 0.7 alcanzando la unidad a $350 \mathrm{~K}$ (ver Figura 8.1 (izquierda)), los cuales no se correponden con los estados de carga mostrados en la Tabla 8.4. Entonces, desde esta comparación no es posible asignarle un estado de carga determinado a dicha 
interacción. Por lo tanto, con la finalidad de interpretar la variación de los parámetros hiperfinos de HFI2 en función de la temperatura, se realizaron cálculos en función del estado de carga variando la carga agregada a la celda con una resolución de $0.1 e^{-}$.

\begin{tabular}{|c|c|c|c|c|c|}
\hline $\begin{array}{c}\text { Estados de } \\
\text { carga de la } \\
\text { celda } \\
\end{array}$ & Aprox. & $d_{N N}(\AA)$ & $V_{33}$ & $\eta$ & Dir. $V_{33}$ \\
\hline \multirow{3}{*}{ Neutra } & LDA & $\begin{array}{l}2.11 \\
2.13 \\
\end{array}$ & +5.19 & 0.49 & {$\left[\begin{array}{lll}1 & -1 & 0\end{array}\right]$} \\
\hline & CW-GGA & $\begin{array}{l}2.10 \\
2.14\end{array}$ & +5.02 & 0.34 & {$\left[\begin{array}{lll}1 & -1 & 0\end{array}\right]$} \\
\hline & GGA & $\begin{array}{l}2.10 \\
2.14 \\
\end{array}$ & +4.77 & 0.26 & {$\left[\begin{array}{lll}1 & -1 & 0\end{array}\right]$} \\
\hline \multirow{3}{*}{ Semi-Cargada } & LDA & $\begin{array}{l}2.13 \\
2.16 \\
\end{array}$ & +5.93 & 0.56 & {$\left[\begin{array}{lll}1 & -1 & 0\end{array}\right]$} \\
\hline & CW-GGA & $\begin{array}{l}2.12 \\
2.16\end{array}$ & +5.86 & 0.49 & {$\left[\begin{array}{lll}1 & -1 & 0\end{array}\right]$} \\
\hline & GGA & $\begin{array}{l}2.12 \\
2.16 \\
\end{array}$ & +6.17 & 0.55 & {$\left[\begin{array}{lll}1 & -1 & 0\end{array}\right]$} \\
\hline \multirow{3}{*}{ Cargada } & LDA & $\begin{array}{l}2.15 \\
2.20\end{array}$ & +5.47 & 0.14 & {$\left[\begin{array}{lll}1 & -1 & 0\end{array}\right]$} \\
\hline & CW-GGA & $\begin{array}{l}2.15 \\
2.20 \\
\end{array}$ & +5.73 & 0.21 & {$\left[\begin{array}{lll}1 & -1 & 0\end{array}\right]$} \\
\hline & GGA & $\begin{array}{l}2.15 \\
2.20 \\
\end{array}$ & +5.79 & 0.25 & {$\left[\begin{array}{lll}1 & -1 & 0\end{array}\right]$} \\
\hline Modelo & Celda & $\boldsymbol{d}_{N N}$ & $V_{33}$ & $\eta$ & Dir. $V_{33}$ \\
\hline PCM & No relajada & $\begin{array}{l}2.05 \\
2.06 \\
\end{array}$ & +5.21 & 0.40 & {$\left[\begin{array}{lll}1 & -1 & 0\end{array}\right]$} \\
\hline PCM & Relajada & $\begin{array}{l}2.15 \\
2.20 \\
\end{array}$ & -5.56 & 0.95 & {$\left[\begin{array}{lll}1 & -1 & 0\end{array}\right]$} \\
\hline Interacción & $\mathbf{T}(\mathbf{K})$ & $f(\%)$ & $V_{33}$ & $\eta$ & Dir. $V_{33}$ \\
\hline HFI1 & 250 & $77(3)$ & $5.56(3)$ & $0.16(2)$ & - \\
\hline HFI2 & 250 & $23(3)$ & $7.72(3)$ & $0.55(1)$ & - \\
\hline HFI1 & 295 & $50.6(3)$ & $5.51(5)$ & $0.26(2)$ & - \\
\hline HFI2 & 295 & $49.4(3)$ & $6.86(5)$ & $0.70(1)$ & - \\
\hline HFI1 & 1123 & $95(1)$ & $5.68(1)$ & $0.13(1)$ & - \\
\hline HFI2 & 1123 & $5(1)$ & $7.06(1)$ & $0.48(3)$ & - \\
\hline
\end{tabular}

Tabla 8.4: Resultados PAC, PCM utilizando las posiciones atómicas de la celda relajada y no relajada y de cálculos $a b$ initio del GCE observado por la impureza Cd en el sitio catiónico del $\mathrm{SnO}_{2}$. Las predicciones APW+lo de $d_{N N}, \mathrm{~V}_{33}$ (dirección y magnitud) y $\eta$ se detallan en función del estado de carga de la celda en las aproximaciones LDA y CW-GGA. Las distancias $d_{N N}$ están dadas en $\AA$ y $\mathrm{V}_{33}$ en unidades de $10^{21} \mathrm{~V} / \mathrm{m}^{2}$. 
En la Figura 8.1 (derecha) se grafican $V_{33}$ y $\eta$ calculados en la aproximación GGA en función del número de electrones agregados a la celda neutra. Contrastando la figura 8.1 (izquierda) y 8.1 (derecha) se puede apreciar que a altas temperaturas la medida de $\mathrm{V}_{33}$ de HFI2 se corresponde con los cálculos APW+lo donde se han agregado entre 1.5 y 1.9 electrones a la celda neutra, los cuales se localizan en la vecindad del Cd. A medida que baja la temperatura $V_{33}$ (de HFI2) se reduce desde 7.4 hasta $6.5 \times 10^{21} \mathrm{~V} / \mathrm{m}^{2}$ aproximadamente, lo que se correlaciona con un estado de carga entre 1 y 1.2 electrones agregados en el entorno de la impureza. Entre 600 y $1000 \mathrm{~K}, \mathrm{~V}_{33}$ de HFI2 se mantiene cercano a este valor, por lo que el estado de carga asignado se mantiene constante en dicho rango de temperaturas. Si bien el parámetro de asimetría a altas temperaturas presenta una disparidad del 20\% con los estados de carga asignados, en el rango 600 $1000 \mathrm{~K}$ la diferencia es menor del $10 \%$. Al bajar la temperatura de 600 a $500 \mathrm{~K}, \mathrm{~V}_{33}$ aumenta levemente de 6.5 a $7 \times 10^{21} \mathrm{~V} / \mathrm{m}^{2}$ lo que se correlaciona con un aumento de la densidad electrónica en el entorno del Cd. Luego, al reducirse la temperatura desde 500 hasta $350 \mathrm{~K}, \mathrm{~V}_{33}$ se reduce hasta $6 \times 10^{21} \mathrm{~V} / \mathrm{m}^{2}$ con lo que el número de electrones agregados cercanos al Cd se reducen a uno. Finalmente, al reducir la temperatura desde 350 a $10 \mathrm{~K}, \mathrm{~V}_{33}$ aumenta y se mantiene relativamente constante por encima de $7.2 \times 10^{21}$ $\mathrm{V} / \mathrm{m}^{2}$ lo aproximadamente constante por debajo de los $300 \mathrm{~K}$ por lo que se infiere que el número de electrones agregados en la vecindad de la impureza está entre 1.5 y 1.9.

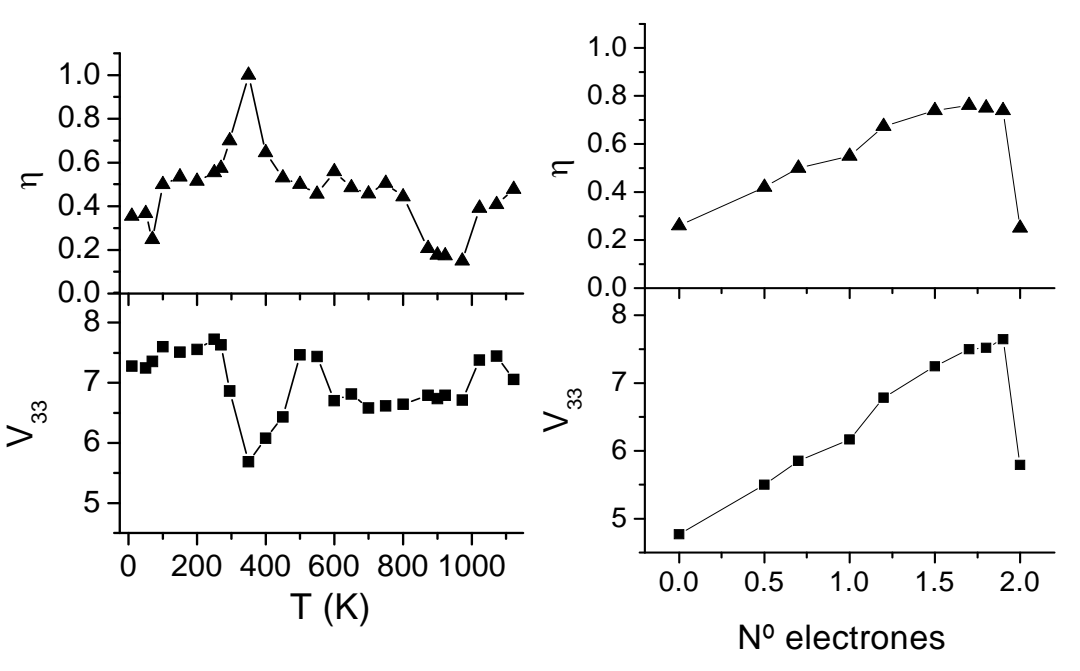

Figura 8.1: (izquierda) $V_{33}$ y $\eta$ vs. $T$ correspondientes a la interacción HFI2. (derecha) $\mathrm{V}_{33}$ y $\eta$ vs. $\mathrm{N}^{\mathrm{o}}$ de electrones agregados a la celda neutra del $\mathrm{SnO}_{2} . \mathrm{V}_{33}$ viene dado en unidades de $10^{21} \mathrm{~V} / \mathrm{m}^{2}$. 
Por lo tanto, a partir del excelente acuerdo entre el cálculo correspondiente a celda cargada y los parámetros hiperfinos de interacción HFI1, se concluye que HFI1 (mayoritaria a altas y bajas temperaturas) corresponde a impurezas Cd con estado de carga cargado, es decir con los dos electrones (agregados a la celda neutra) localizados en el entorno del Cd y sus primeros vecinos oxígeno. En cuanto a la segunda interacción (HFI2)la cual se hace mayoritaria en una pequeña ventana de temperatura a temperaturas intermedias y presenta una gran variación en sus parámetros hiperfinos $\mathrm{V}_{33}$ y $\eta$. Esta interacción fue totalmente interpretada a partir de un estudio APW+lo del GCE variando la carga con una resolución de $0.1 e^{-}$, con lo que se concluye que los cambios observados en $V_{33}$ y $\eta$ de HFI2 se deben a que las cargas que ionizan los huecos electrónicos (responsables del proceso dinámico observado) cambian en función de la temperatura dando lugar a cambios en el GCE final estable (de HFI2) conforme cambia la temperatura.

Desde la comparación entre los resultados PAC y los cálculos PCM para el caso de celda no relajada se aprecia un total desacuerdo con la segunda interacción HFI2, mientras que para HFI1 si bien la componente $V_{33}$ predicha por PCM discrepa aproximadamente en un $10 \%$ del valor experimental, el parámetro $\eta$ presenta diferencias del orden del 50\%. Si consideramos las relajaciones predichas por FPAPW+lo (ver Apéndice III), vuelve a observarse un total desacuerdo con HFI2, mientras que con HFI1 a pesar que $\mathrm{V}_{33}$ calculado con PCM está en muy buen acuerdo el valor experimental, el parámetro $\eta$ difiere en más del 70\%. Por lo tanto, se concluye que PCM no es capaz de describir satisfactoriamente las interacciones hiperfinas observadas por la impureza Cd en el sitio catiónico del $\mathrm{SnO}_{2}$.

\section{8-3 Comparación de resultados PAC con cálculos APW+lo en ZnO: ${ }^{111} \mathrm{Cd}$}

En este apartado se comparan los resultados PAC y los cálculos ab initio realizados en el semiconductor de $\mathrm{ZnO}$ dopado con Cd. En la Tabla 8.5 se comparan los parámetros hiperfinos que caracterizan el GCE en el sitio de la sonda-impureza Cd medidos con la técnica PAC, calculados con el modelo PCM y calculados con el método APW+lo. Se puede apreciar que tanto las medidas de $\mathrm{V}_{33} \mathrm{y} \eta$ realizadas en todo el rango de temperaturas (y en particular las tabuladas a $1075 \mathrm{~K}$ y a temperatura 
ambiente) están en excelente con los parámetros calculados en celda neutra con las aproximaciones GGA y CW-GGA, mientras que el acuerdo con las predicciones LDA resulta relativamente bueno también para el caso de celda neutra. A partir de la figura 5.14 (Capítulo 5) se puede observar que los parámetros hiperfinos de la única interacción ajustada se mantienen constantes en todo el rango de temperaturas, por lo que el estado final estable alcanzado por el Cd luego del proceso dinámico resulta ser el correspondiente a celda neutra. Cabe destacar que debido a que el $\mathrm{Cd}^{2+}$ es isovalente al catión $\mathrm{Zn}^{2+}$ en $\mathrm{ZnO}$, el estado final estable, que será el de celda neutra, que alcanza la sonda Cd luego del proceso de AE no se encuentra ionizado en todo el rango de temperatura.

Por lo tanto, a partir del excelente acuerdo observado entre los parámetros hiperfinos de la única interacción ajustada y los cálculos FP-APW+lo se concluye que la interacción hiperfina observada corresponde a impurezas Cd localizadas en el sitio catiónico del $\mathrm{ZnO}$, las cuales no se encuentran ionizadas en todo el rango de temperatura.

Desde la comparación entre los resultados experimentales y las predicciones PCM se observa que $\mathrm{V}_{33}$ difiere en un $40 \%$ para el caso de celda no relajada, mientras que si consideramos las relajaciones producidas por el Cd en la red del semiconductor calculadas con el método FP-APW+lo (ver Apéndice III) se observa un diferencia del 70\%. El acuerdo entre los parámetros de asimetría se debe a la simetría puntual del sitio catiónico del ZnO. Por lo tanto, el modelo PCM es obsoleto para predecir las interacciones observadas por la impureza Cd en la red del ZnO. 


\begin{tabular}{|c|c|c|c|c|c|}
\hline $\begin{array}{c}\text { Estados de } \\
\text { carga de la } \\
\text { celda } \\
\end{array}$ & Aprox. & $d_{N N}(\AA)$ & $V_{33}$ & $\eta$ & Dir. $V_{33}$ \\
\hline $\begin{array}{c}\text { Descargada } \\
2 \mathrm{e}-\end{array}$ & CW-GGA & $\begin{array}{l}2.16 \\
2.19\end{array}$ & +1.20 & 0.00 & {$\left[\begin{array}{lll}0 & 0 & 1\end{array}\right]$} \\
\hline \multirow{3}{*}{ Descargada } & LDA & $\begin{array}{l}2.16 \\
2.18\end{array}$ & +1.07 & 0.00 & {$\left[\begin{array}{lll}0 & 0 & 1\end{array}\right]$} \\
\hline & CW-GGA & $\begin{array}{l}2.16 \\
2.19\end{array}$ & +1.37 & 0.00 & {$\left[\begin{array}{lll}0 & 0 & 1\end{array}\right]$} \\
\hline & GGA & $\begin{array}{l}2.16 \\
2.19 \\
\end{array}$ & +1.20 & 0.00 & {$\left[\begin{array}{lll}0 & 0 & 1\end{array}\right]$} \\
\hline \multirow{3}{*}{ Neutra } & LDA & $\begin{array}{l}2.16 \\
2.18\end{array}$ & +1.43 & 0.00 & {$\left[\begin{array}{lll}0 & 0 & 1\end{array}\right]$} \\
\hline & CW-GGA & $\begin{array}{l}2.16 \\
2.19\end{array}$ & +1.68 & 0.00 & {$\left[\begin{array}{lll}0 & 0 & 1\end{array}\right]$} \\
\hline & GGA & $\begin{array}{l}2.16 \\
2.19 \\
\end{array}$ & +1.69 & 0.00 & {$\left[\begin{array}{lll}0 & 0 & 1\end{array}\right]$} \\
\hline \multirow{3}{*}{ Cargada } & LDA & $\begin{array}{l}2.16 \\
2.18\end{array}$ & +1.30 & 0.00 & {$\left[\begin{array}{lll}0 & 0 & 1\end{array}\right]$} \\
\hline & CW-GGA & $\begin{array}{l}2.16 \\
2.19 \\
\end{array}$ & +1.79 & 0.00 & {$\left[\begin{array}{lll}0 & 0 & 1\end{array}\right]$} \\
\hline & GGA & $\begin{array}{l}2.16 \\
2.19 \\
\end{array}$ & +1.78 & 0.00 & {$\left[\begin{array}{lll}0 & 0 & 1\end{array}\right]$} \\
\hline Modelo & Celda & $\boldsymbol{d}_{N N}$ & $V_{33}$ & $\eta$ & Dir. $V_{33}$ \\
\hline PCM & No relajada & $\begin{array}{l}1.97 \\
1.99 \\
\end{array}$ & +0.94 & 0.00 & {$\left[\begin{array}{lll}0 & 0 & 1\end{array}\right]$} \\
\hline PCM & Relajada & $\begin{array}{l}2.16 \\
2.19 \\
\end{array}$ & +0.45 & 0.00 & {$\left[\begin{array}{lll}0 & 0 & 1\end{array}\right]$} \\
\hline Método & $T(K)$ & $f(\%)$ & $V_{33}$ & $\eta$ & Dir. $V_{33}{ }^{(a)}$ \\
\hline PAC & 295 & 100 & $1.63(1)$ & $0.11(3)$ & {$\left[\begin{array}{lll}0 & 0 & 1\end{array}\right]$} \\
\hline PAC & 1075 & 100 & $1.60(1)$ & $0.08(3)$ & {$\left[\begin{array}{lll}0 & 0 & 1\end{array}\right]$} \\
\hline
\end{tabular}

Tabla 8.5: Resultados PAC, PCM utilizando las posiciones atómicas de la celda relajada y no relajada y de los cálculos $a b$ initio del GCE observado por la impureza Cd en el sitio catiónico del $\mathrm{ZnO}$. Las predicciones APW+lo de $d_{N N}, \mathrm{~V}_{33}$ (dirección y magnitud) y $\eta$ se detallan en función del estado de carga de la celda en las aproximaciones LDA y CW-GGA. Las distancias $d_{N N}$ están dadac on $\AA_{\mathrm{V}} \mathrm{V} / m$ on unidadoc do $1 \mathrm{n}^{21} \mathrm{~V} / \mathrm{m}^{2}$

(a) Las direcciones experimentales corresponden a monocistales de $\mathrm{ZnO}$ medidos por Rita et al. [Rita 2005]. 


\section{8-4 Comparación de resultados PAC con cálculos APW+lo en SnO: ${ }^{111} \mathrm{Cd}$}

En esta sección se comparan los resultados experimentales y teóricos realizados en el semiconductor SnO dopado con impurezas ${ }^{111} \mathrm{Cd}$. En la Tabla 8.6 se detallan los parámetros hiperfinos medidos con la técnica PAC correspondientes a la impureza ${ }^{111} \mathrm{Cd}$ localizada en el sitio catiónico, las predicciones PCM y el cálculo del GCE en función del estado de carga de la celda con el método APW+lo. Se puede observar que la componente $\mathrm{V}_{33}$ y el parámetro $\eta$ de la interacción HFI1 medida a $900 \mathrm{~K}$ se encuentra en muy buen acuerdo con el cálculo APW+lo-LDA correspondiente a la celda neutra, mientras que la segunda interacción (HFI2) observada a la misma temperatura está en buen acuerdo con el cálculo APW+lo (LDA) con estado de carga descargado. Por otro lado, se aprecia que los resultados obtenidos con la aproximación CW-GGA no pueden ser correlacionados con las medidas PAC tabuladas.

En la Figura 5.4 del Capítulo 5 se puede observar que las dos interacciones (HFI1 y HFI2) son estables en todo el rango de temperaturas. La interacción HFI2, que no presenta atenuación dinámica, es compatible con la situación de la impureza ${ }^{111} \mathrm{Cd}$ con un hueco electrónico atrapado en su entorno (celda descargada). HFI1 se correlaciona con el ${ }^{111} \mathrm{Cd}$ sin huecos electrónicos atrapados (celda neutra) y como la componente principal $V_{33}$ calculada en el sitio del $\mathrm{Cd}$ se ve modificada en un $50 \%$ aproximadamente al sacar un electrón de la celda neutra, se infiere que dicho electrón está lo suficientemente cerca de la impureza como para producir dicha variación. Teniendo en cuenta que la impureza $\mathrm{Cd}^{2+}$ es isovalente al catión nativo $\mathrm{Sn}^{2+}$, entonces la interacción HFI1 corresponde a sondas ${ }^{111} \mathrm{Cd}$ no ionizadas mientras que HFI2 se correlaciona con sondas ${ }^{111} \mathrm{Cd}$ ionizadas (sin un electrón). Finalmente, de la figura 5.4 se observa también que en el entorno de las sondas ${ }^{111} \mathrm{Cd}$ que experimentan la interacción HFI1 a altas temperaturas, el hueco electrónico es ocupado rápidamente por un electrón ( $\lambda_{\mathrm{g}}$ grande, $\tau_{\mathrm{g}}$ pequeña) por lo que el estado final estable es alcanzado antes de que la atenuación sea apreciable, mientras que por debajo de $700 \mathrm{~K}$ el estado final es alcanzado más lentamente ( $\lambda_{\mathrm{g}}$ pequeña, $\tau_{\mathrm{g}}$ grande) haciéndose bien apreciable la atenuación dinámica. 


\begin{tabular}{|c|c|c|c|c|c|}
\hline Estado de carga & Aprox. & $d_{N N}(\AA)$ & $V_{33}$ & $\eta$ & Dir. $V_{33}$ \\
\hline \multirow{2}{*}{ Descargada } & LDA & 2.26 & +7.79 & 0.00 & {$\left[\begin{array}{lll}0 & 0 & 1\end{array}\right]$} \\
\hline & CW-GGA & 2.28 & +5.22 & 0.00 & {$\left[\begin{array}{lll}0 & 0 & 1\end{array}\right]$} \\
\hline \multirow{2}{*}{ Neutra } & LDA & 2.27 & +5.26 & 0.00 & {$\left[\begin{array}{lll}0 & 0 & 1\end{array}\right]$} \\
\hline & CW-GGA & 2.31 & +1.93 & 0.00 & {$\left[\begin{array}{lll}0 & 0 & 1\end{array}\right]$} \\
\hline \multirow{2}{*}{ Cargada } & LDA & 2.37 & -4.52 & 0.00 & {$\left[\begin{array}{lll}0 & 0 & 1\end{array}\right]$} \\
\hline & CW-GGA & 2.37 & -4.13 & 0.00 & {$\left[\begin{array}{lll}0 & 0 & 1\end{array}\right]$} \\
\hline Modelo & Celda & $\boldsymbol{d}_{N N}$ & $V_{33}$ & $\eta$ & $\begin{array}{l}\text { Dir. } \\
V_{33} \\
\end{array}$ \\
\hline PCM & No relajada & 2.22 & +5.45 & 0.00 & {$\left[\begin{array}{lll}0 & 0 & 1\end{array}\right]$} \\
\hline PCM & Relajada & 2.31 & +3.69 & 0.00 & {$\left[\begin{array}{lll}0 & 0 & 1\end{array}\right]$} \\
\hline Interacción & $T(K)$ & $f(\%)$ & $V_{33}$ & $\eta$ & Dir. $V_{33}$ \\
\hline HFI1 & 298 & $97.7(5.0)$ & $5.35(4)$ & $0.29(1)$ & - \\
\hline HFI2 & 298 & $2.3(5.0)$ & $7.50(5)$ & $0.19(4)$ & - \\
\hline HFI1 & 900 & $77.7(5.3)$ & $5.69(1)$ & $0.11(1)$ & - \\
\hline HFI2 & 900 & $22.3(5.3)$ & $6.78(2)$ & $0.06(3)$ & - \\
\hline
\end{tabular}

Tabla 8.6: Resultados PAC, PCM utilizando las posiciones atómicas de la celda relajada y no relajada y de cálculos ab initio del GCE observado por la impureza ${ }^{111} \mathrm{Cd}$ en el sitio catiónico del SnO. Las predicciones APW+lo de $d_{N N}, V_{33}$ (dirección y magnitud) y $\eta$ se detallan en función del estado de carga de la celda en las aproximaciones LDA y CWGGA. Las distancias $d_{N N}$ están dadas en $\AA$ y $V_{33}$ en unidades de $10^{21} \mathrm{~V} / \mathrm{m}^{2}$.

Por lo tanto, desde el excelente acuerdo entre las 2 interacciones hiperfinas observadas y los cálculos APW+lo realizados con la aproximación LDA se concluye que la interacción HFI1 corresponde a la impureza ${ }^{111} \mathrm{Cd}$ no ionizada localizada en el sitio catiónico del semiconductor SnO, mientras que la interacción HFI2 corresponde a la impureza ${ }^{111} \mathrm{Cd}$ ionizada sin un electrón localizada en el sitio catiónico del SnO.

Desde la comparación entre las medidas experimentales y las predicciones PCM se aprecia un buen acuerdo entre los cálculos PCM correspondientes a celda no relajada y la interacción mayoritaria HFI1, mientras que respecto a la comparación con HFI2 se observa una diferencia del 25\%. Sin embargo, es necesario considerar las relajaciones 
producidas de la impureza en el son predichas por el método FP-APW+lo (ver Apéndice III). Desde la comparación entre los resultados PAC y las predicciones PCM correspondientes a celda relajada, se aprecia un desacuerdo del 30\% con HFI1 y del $50 \%$ con HFI2 en la componente $\mathrm{V}_{33}$. Por lo tanto, se concluye que PCM resulta ser obsoleto a la hora de describir las interacciones hiperfinas observadas por el Cd en el SnO.

\section{8-5 Localización de los estados electrónicos del nivel de impureza}

En este apartado se analiza la localización espacial de los electrones que contribuyen al nivel de impureza de cada sistema estudiado. Para esto se graficó la densidad electrónica $\rho(r)$ pero solo de los estados electrónicos que contribuyen al nivel de impureza. Entonces, se integró la densidad $\rho(\boldsymbol{r})$ entre la energía más baja del nivel de impureza y del nivel de Fermi. Finalmente, se graficaron las curvas de nivel de $\rho(\boldsymbol{r})$ donde $r$ está restringido a dos dimensiones, estos planos fueron elegidos de modo que corten las regiones de $R^{3}$ donde la densidad electrónica era apreciable.

Primero se muestra la localización de electrones en las bixbitas estudiadas. Se analizan los casos del Cd localizado en los sitios C y D.

\section{8-5-1 Localización electrónica en el óxido $\operatorname{In}_{2} \mathrm{O}_{3}: \mathrm{Cd}$}

\section{Cd en sitio D:}

Para el caso de Cd en el sitio D de la estructura Bixbita se eligieron dos planos característicos para graficar la densidad electrónica. Los planos elegidos son los que contienen los oxígenos $\mathrm{O}_{\mathrm{NN}}$ del Cd debido a que tal como se demostró en la sección 72-3 del Capítulo 7, el electrón agregado a la celda neutra se sitúa en la vecindad de la impureza. En la Figura 8.2 (a) se grafica la celda de cálculo de $\operatorname{In}_{2} \mathrm{O}_{3}$ :Cd donde sólo se representan las esferas atómicas que se sitúan en el plano (1 -1 0$)$ que pasa por el centro y contiene las impurezas Cd localizadas en cuatro vértices de la celda (esferas de color magenta). Cabe destacar que además se agregaron los $\mathrm{O}_{\mathrm{NN}}$ del $\mathrm{Cd}$ los cuales se muestran con ligaduras de color gris, donde dos de los $6 \mathrm{O}_{\mathrm{NN}}$ se encuentran sobre el plano representado. En la Figura 8.2 (b) se muestra el plano (1 110$)$ que pasa por el centro de la celda y contiene las impurezas Cd situadas en los vértices del cubo que se 
encuentran sobre el plano, y además contiene los cuatro primeros vecinos $\mathrm{O}_{\mathrm{NN}}$ restantes (del Cd). Con un vector verde se representa la componente principal $V_{33}$ en la dirección [1 111 1] de dicha componente.

En la Figura 8.3 (a) se grafican las curvas de nivel de la densidad electrónica correspondiente al electrón agregado a la celda neutra del $\operatorname{In}_{2} \mathrm{O}_{3}: \mathrm{Cd}$ en el entorno del átomo de Cd y sus vecinos $\mathrm{O}_{\mathrm{NN}}$ sobre el plano (1 -1 0) y en la Figura 8.3 (b) sobre el plano (1 110$)$.

Se puede apreciar que sobre el plano (1 - 10$)$ el electrón se localiza en la impureza Cd y en sus vecinos $\mathrm{O}_{\mathrm{NN}}$, mientras que en el plano (lllll 110 ) se observa que la densidad electrónica es también apreciable en los dos oxígenos segundos vecinos $\left(\mathrm{O}_{\mathrm{NNN}}\right.$ por next nearest neighbors).

Por lo tanto, desde el análisis de la Figura 8.3 y desde lo discutido en la sección 7-23 (a) donde se dedujo que el 60\% de los estados electrónicos que contribuyen al nivel de impureza se localizan sobre las esferas del Cd y de sus primeros vecinos oxígeno, se concluye que el electrón agregado a la celda neutra se encuentra esencialmente en las ligaduras $\mathrm{Cd}-\mathrm{O}_{\mathrm{NN}}$.

(a)

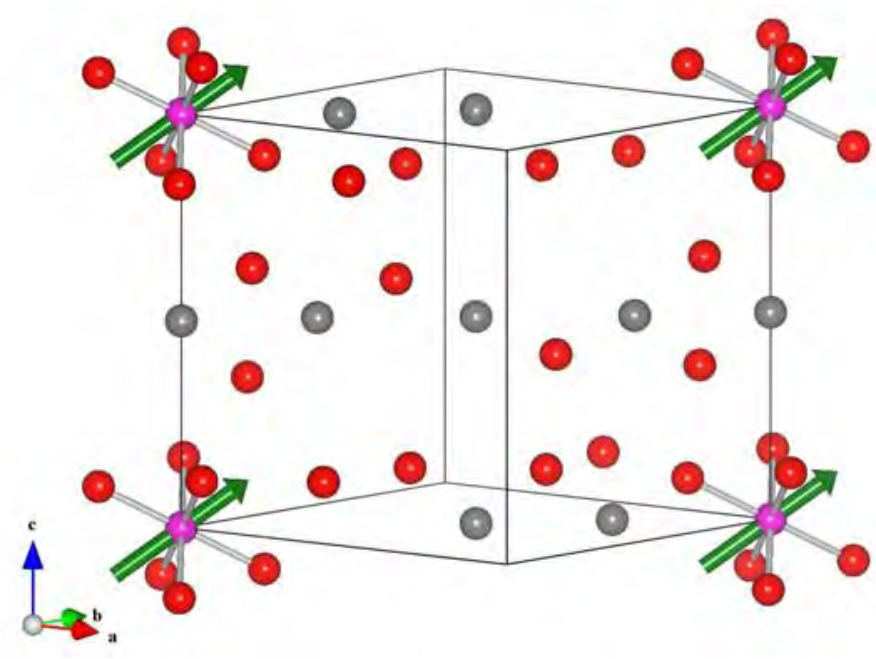

(b)

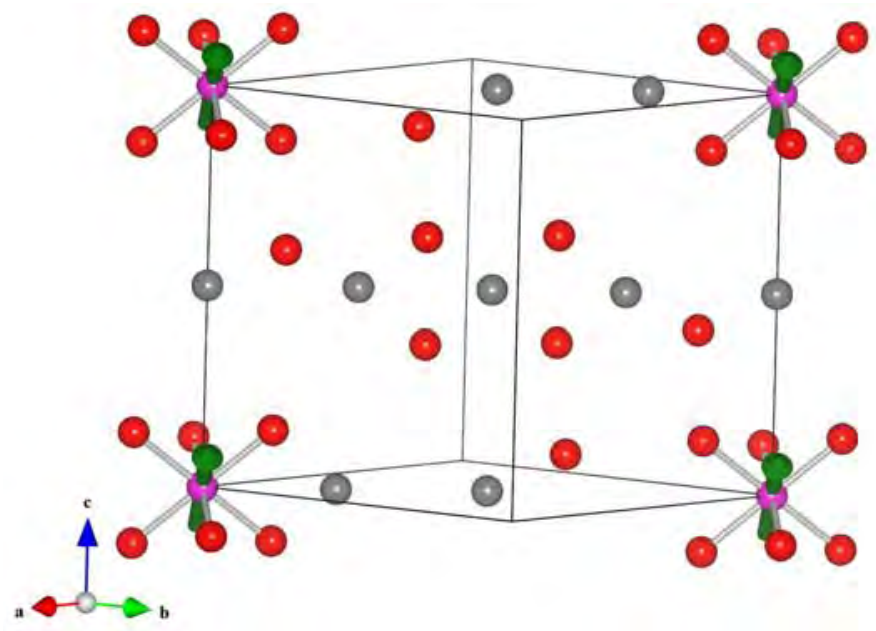

Figura 8.2: Átomos contenidos en los planos: (a) (1 $\left.\begin{array}{lll}1 & 0\end{array}\right)$; (b) $\left(\begin{array}{lll}1 & 1 & 0\end{array}\right)$ de la celda unidad del $\mathrm{In}_{2} \mathrm{O}_{3}$ :Cd. Las impurezas Cd están localizadas en el sitio D de la estructura bixbita (vértices de la celda). Con esferas magenta se representan las impurezas $\mathrm{Cd}$, con esferas rojas los oxígenos y con esferas grises los cationes. El vector verde simboliza la dirección [ll 111 1] de la componente principal $\mathrm{V}_{33}$. 
(a)

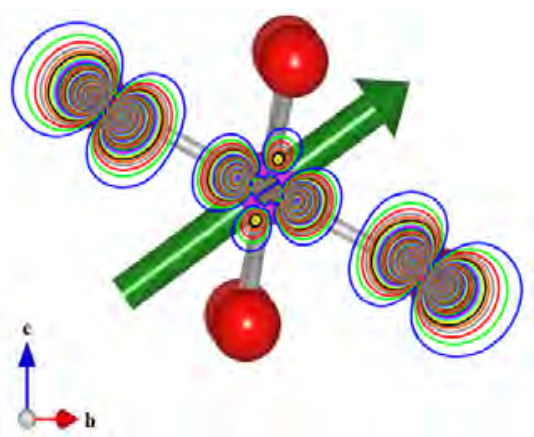

Figura 8.3: Curvas de nivel de la densidad de carga del electrón agregado a la celda neutra de $\mathrm{In}_{2} \mathrm{O}_{3}$ : (a) sobre el plano (1 -10$)$ que contiene al $\mathrm{Cd}$ y dos de sus vecinos $\mathrm{O}_{\mathrm{NN}}$; (b) sobre el plano (1 110$)$ que contiene al Cd y sus restantes vecinos $\mathrm{O}_{\mathrm{NN}}$. El paso utilizado en las curvas de nivel es de $1 \%$ relativo al máximo. (b)
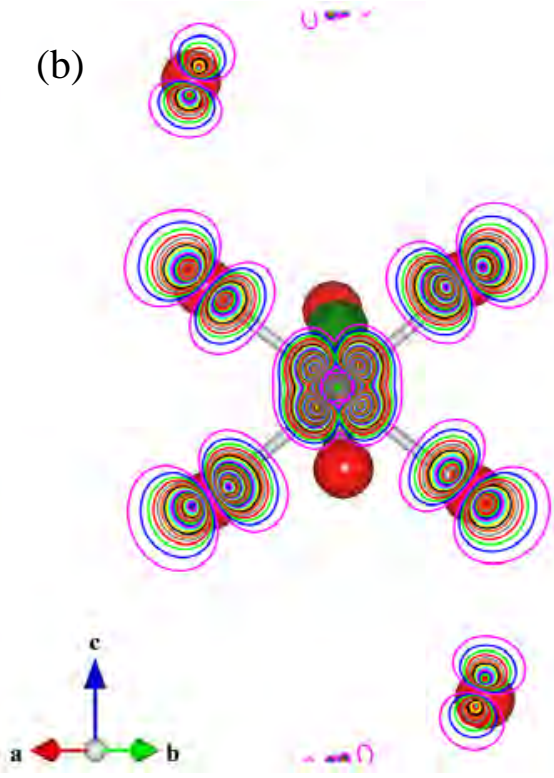

\section{Cd en sitio C:}

Ahora analizamos la densidad electrónica para el caso de Cd en el sitio C. Al igual que en el caso de Cd en el sitio $\mathrm{D}$, se seleccionaron dos planos donde se proyectó la densidad electrónica. Tales planos son los que contienen a los primeros vecinos $\mathrm{O}_{\mathrm{NN}} \mathrm{y}$, al igual que en el caso anterior, el electrón se localiza cercano a la impureza (ver sección 7-2-3 (a) del Capítulo 7). En la Figura 8.4 (a) se grafica el plano (0 0 1) que pasa por los vecinos $\mathrm{O}_{\mathrm{NN} 2}$ de la impureza $\mathrm{Cd}$ la cual se localiza en 4 de los vértices de la celda. Además, se muestran los átomos que se localizan sobre el plano y se representan los 6 vecinos $\mathrm{O}_{\mathrm{NN}}$ del $\mathrm{Cd}$ (los $\mathrm{O}_{\mathrm{NN} 2}$ sobre el plano, mientras que los $\mathrm{O}_{\mathrm{NN} 1}$ y los $\mathrm{O}_{\mathrm{NN} 3}$ fuera del plano). En la Figura 8.4 (b) se representa el plano (1 10 ), el cual pasa por las impurezas Cd localizadas en 4 de los vértices de la celda y adicionalmente contiene los vecinos oxígeno $\mathrm{O}_{\mathrm{NN} 1}$ y $\mathrm{O}_{\mathrm{NN} 3}$, los restantes vecinos $\mathrm{O}_{\mathrm{NN} 2}$ representados en la figura se encuentran fuera del plano. 
(a)

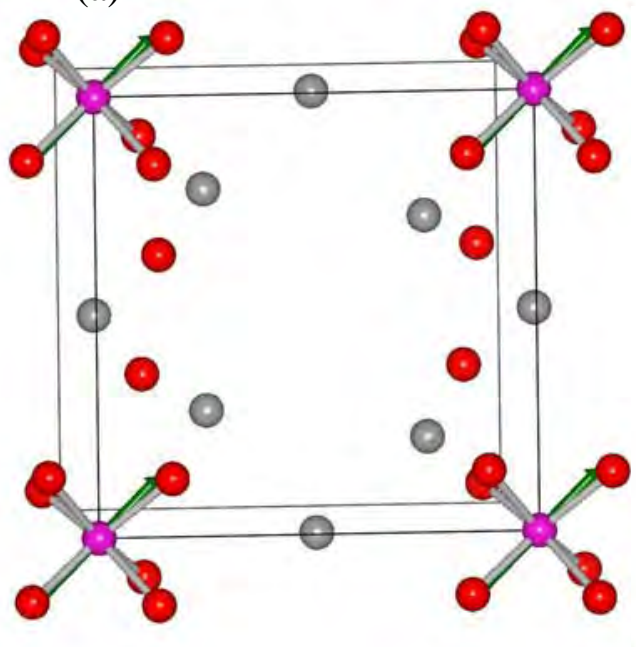

(b)

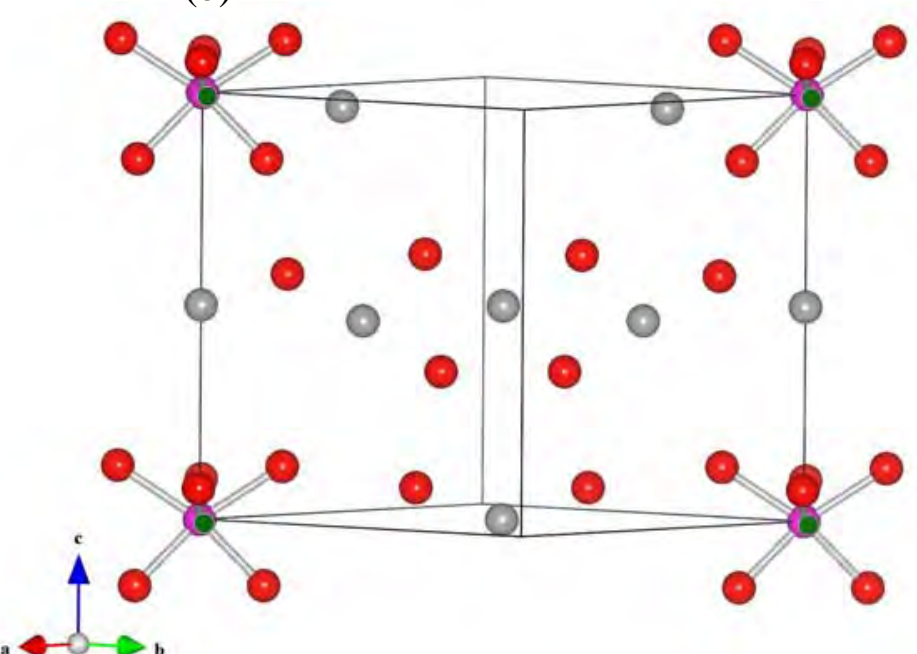

Figura 8.4: Átomos contenidos en los planos: (a) ( $\left.\begin{array}{lll}0 & 0 & 1\end{array}\right)$; (b) $\left(\begin{array}{lll}1 & 1 & 0\end{array}\right)$ de la celda unidad del $\mathrm{In}_{2} \mathrm{O}_{3}: \mathrm{Cd}$. Las impurezas $\mathrm{Cd}$ se localizan en 4 de los vértices de la celda (sitio $\mathrm{C}$ de la estructura bixbita). Con esferas magenta se representan las impurezas $\mathrm{Cd}$, con esferas rojas los oxígenos y con esferas grises los cationes. El vector verde simboliza la dirección [1 110$]$ de la componente principal $\mathrm{V}_{33}$ para el caso de celda cargada.

En la Figura 8.5 (a) se grafican las curvas de nivel de la densidad electrónica del electrón agregado a la celda neutra sobre el plano $\left(\begin{array}{lll}0 & 0 & 1\end{array}\right)$ que pasa por la impureza Cd (esfera magenta) y los primeros vecinos $\mathrm{O}_{\mathrm{NN} 2}$, además se representan las esferas de los restantes $\mathrm{O}_{\mathrm{NN}}$ (todos los átomos oxígeno se representan con esferas rojas), la dirección $\left[\begin{array}{lll}1 & 1 & 0\end{array}\right]$ de $\mathrm{V}_{33}$ (vector verde) y las ligaduras $\mathrm{Cd}-\mathrm{O}_{\mathrm{NN}}$ (bastones grises). En la Figura 8.5 (b) se proyectó la densidad del electrón sobre el plano $\left(\begin{array}{lll}1 & 1 & 0\end{array}\right)$ que pasa la impureza Cd y los vecinos $\mathrm{O}_{\mathrm{NN} 1}$ y $\mathrm{O}_{\mathrm{NN} 3}$. Se puede apreciar que como en el caso de Cd localizado en el sitio D, el electrón se localiza esencialmente en el entorno de la impureza Cd y sus primeros vecinos $\mathrm{O}_{\mathrm{NN}}$. Esto está en perfecto acuerdo con lo discutido en la sección 7-23 (a) para el caso del Cd en el sitio C donde se dedujo que el 70\% de los estados que contribuyen al nivel de impureza corresponden a las esferas del Cd y sus vecinos $\mathrm{O}_{\mathrm{NN}}$. Sin embargo, se puede apreciar que la probabilidad de encontrar al electrón es apreciablemente mayor sobre los $\mathrm{O}_{\mathrm{NN} 1}$ y $\mathrm{O}_{\mathrm{NN} 3}$ que se sitúan sobre el plano $\left(\begin{array}{lll}1 & 1 & 0\end{array}\right)$ respecto a los átomos oxígeno $\mathrm{O}_{\mathrm{NN} 2}$ que están sobre el plano $\left(\begin{array}{lll}0 & 0 & 1\end{array}\right)$. Recordemos que la multiplicidad de los primeros vecinos oxígeno $\mathrm{O}_{\mathrm{NN} i}(i=1,2,3)$ del Cd es 2. 
Esto se correlaciona con la contribución de los oxígenos primeros vecinos del Cd (localizado en el sitio C) a la densidad de estados en la región del nivel de impureza tal como se aprecia en la Figura 8.6. Se puede observar que los oxígenos $\mathrm{O}_{\mathrm{NN} 1}$ y $\mathrm{O}_{\mathrm{NN} 3}$ del Cd, los cuales se sitúan sobre el plano (1 10 ), son los que contribuyen mayoritariamente al nivel de impureza respecto a la contribución de los $\mathrm{O}_{\mathrm{NN} 2}$ (localizados sobre el plano $\left(\begin{array}{lll}0 & 0 & 1\end{array}\right)$.

(a)

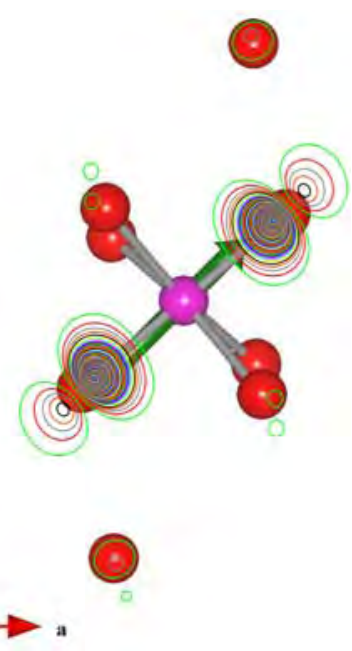

(b)
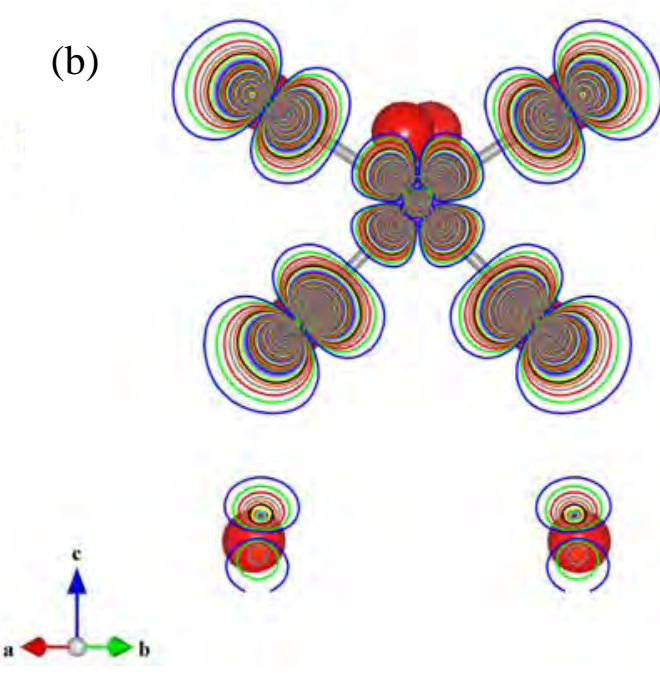

Figura 8.5: Curvas de nivel de la densidad de carga del electrón agregado a la celda neutra de $\mathrm{In}_{2} \mathrm{O}_{3}$ :Cd con el Cd en el sitio C: (a) sobre el plano $\left(\begin{array}{lll}0 & 0 & 1\end{array}\right)$ que contiene los vecinos $\mathrm{O}_{\mathrm{NN} 2}$; (b) sobre el plano ( $\left.\begin{array}{lll}1 & 1 & 0\end{array}\right)$ que contiene al Cd y sus vecinos $\mathrm{O}_{\mathrm{NN} 1}$ y $\mathrm{O}_{\mathrm{NN} 3}$. Con esferas rojas se representan los oxígenos, con esferas magenta el Cd y el vector verde apunta en la dirección [ $\left.\begin{array}{llll}1 & 1 & 0\end{array}\right]$ de V22 para el caso de celda cargada. El paso utilizado en las curvas de nivel es de 1\% relativo al máximo.

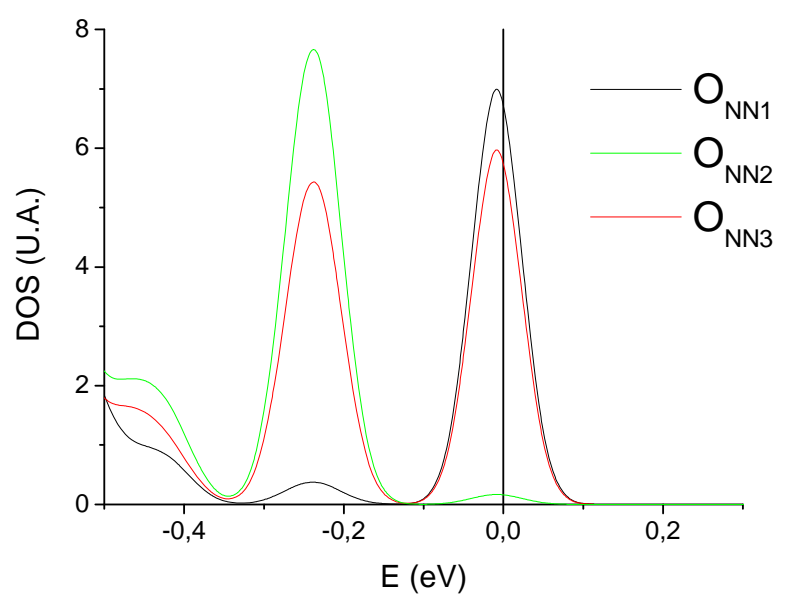

Figura 8.6: Contribución a la DOS en el entorno del nivel de Fermi de los primeros vecinos $\mathrm{O}_{\mathrm{NNi}}(i=1,2,3)$ del Cd localizada en el sitio $\mathrm{C}$ del $\mathrm{In}_{2} \mathrm{O}_{3}$. 


\section{8-5-2 Localización electrónica en el óxido $\mathrm{Y}_{2} \mathrm{O}_{3}$ :Cd}

\section{Cd en sitio D:}

En este apartado se muestra la localización del electrón agregado a la celda neutra de la bixbita $\mathrm{Y}_{2} \mathrm{O}_{3}$ :Cd. Primero se analiza el caso del Cd en el sitio D. Para proyectar la densidad electrónica se utilizaron los planos ( $\left.\begin{array}{lll}1 & -1 & 0\end{array}\right)$ y $\left(\begin{array}{lll}1 & 1 & 0\end{array}\right)$ al igual que en el caso de Cd situado en el sitio D del $\mathrm{In}_{2} \mathrm{O}_{3}$ (ver Figura 8.2).

En la Figura 8.7 (a) se grafican las curvas de nivel de la densidad electrónica proyectada sobre el plano ( 1 - 10$)$ pasante por la impureza Cd y por 2 de sus 6 primeros vecinos oxígeno. En la Figura 8.7 (b) se proyecta la densidad sobre el plano (1 110$)$ que contiene la impureza $\mathrm{Cd}$ y los 4 primeros vecinos $\mathrm{O}_{\mathrm{NN}}$ restantes. $\mathrm{Al}$ igual que en el caso del $\mathrm{Cd}$ en el sitio $\mathrm{D}$ del $\mathrm{In}_{2} \mathrm{O}_{3}$, el electrón se localiza mayoritariamente sobre la impureza Cd y los $\mathrm{O}_{\mathrm{NN}}$ y se aprecia que hay una pequeña probabilidad de encontrarlo en los oxígenos $\mathrm{O}_{\mathrm{NNN}}$ situados sobre el plano (1 110$)$. Este hecho está en acuerdo con lo visto en la sección 7-2-3 (b) donde se dedujo que el 60\% de los estados electrónicos de nivel de impureza se localizan en las esferas del Cd y sus vecinos $\mathrm{O}_{\mathrm{NN}}$.

(a)

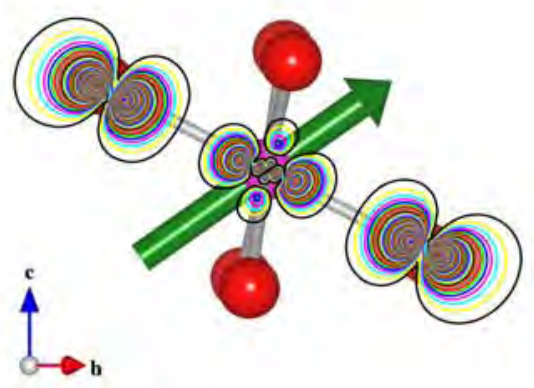

Figura 8.7: Curvas de nivel de la densidad de carga del electrón agregado a la celda neutra de $\mathrm{Y}_{2} \mathrm{O}_{3}$ :Cd: (a) sobre el plano (1 -1 0 ) que pasa por el Cd y dos de sus vecinos $\mathrm{O}_{\mathrm{NN}}$; (b) sobre el plano (1 110$)$ que contiene al Cd y sus cuatro vecinos $\mathrm{O}_{\mathrm{NN}}$ restantes. Con esferas rojas se representan los oxígenos, con esferas magenta el Cd y el vector verde representa la dirección [ [ $\left.\begin{array}{lll}1 & 1 & 1\end{array}\right]$ de $\mathrm{V}_{33}$. El paso utilizado en las curvas de nivel es de $1 \%$ relativo al máximo. (b)

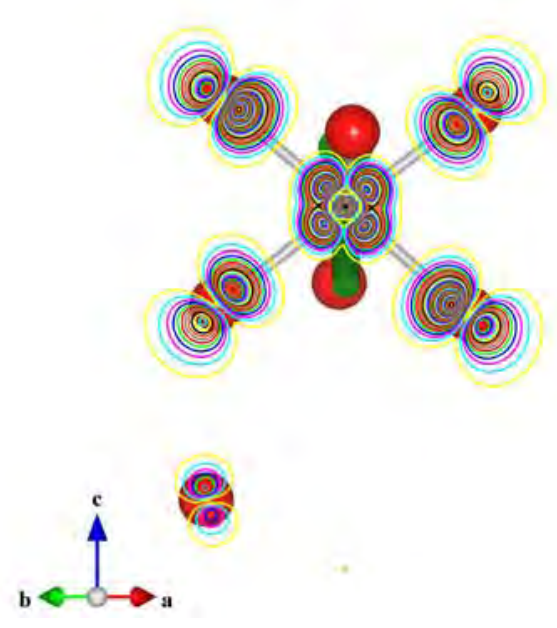




\section{Cd en sitio C:}

En este caso, las curvas de nivel de la densidad de carga se proyectaron sobre los planos ( $\left(\begin{array}{lll}0 & 0 & 1\end{array}\right)$ y $\left(\begin{array}{lll}1 & 1 & 0\end{array}\right)$ de la celda del $\mathrm{Y}_{2} \mathrm{O}_{3}$ :Cd con el Cd localizado en el sitio $\mathrm{C}$ (ver Figura 8.4). En la Figura 8.8 (a) se grafica las curvas de nivel de la densidad electrónica proyectada sobre el plano (l $\left.\begin{array}{lll}0 & 0 & 1\end{array}\right)$ el cual contiene los primeros vecinos $\mathrm{O}_{\mathrm{NN} 2}$ del Cd localizado en el sitio C. En la Figura 8.8 (b) se grafica la proyección de la densidad electrónica sobre el plano ( $\left.\begin{array}{lll}1 & 1 & 0\end{array}\right)$ que contiene a la impureza $\mathrm{Cd}$ y los vecinos $\mathrm{O}_{\mathrm{NN} 1} \mathrm{y}$ $\mathrm{O}_{\mathrm{NN} 3}$.

Análogamente a lo observado en $\mathrm{Cd}$ en el sitio $\mathrm{C}$ de la bixbita $\mathrm{In}_{2} \mathrm{O}_{3}$ y en acuerdo con lo deducido en la sección 7-2-3 (a), se aprecia que el electrón se encuentra localizado mayoritariamente en el Cd y sus vecinos $\mathrm{O}_{\mathrm{NN}}$, mientras que se observa una débil localización en los segundos vecinos $\mathrm{O}_{\mathrm{NNN}}$. Además, se vuelve a apreciar que la densidad electrónica es mayor en los oxígenos $\mathrm{O}_{\mathrm{NN} 1}$ y $\mathrm{O}_{\mathrm{NN} 3}$ situados en el plano $\left(\begin{array}{lll}1 & 1 & 0\end{array}\right)$ respecto a los $\mathrm{O}_{\mathrm{NN} 2}$ del plano (l 001 ). Para interpretar este comportamiento, en la Figura 8.9 se grafican las contribuciones parciales a la densidad de estados de los tres pares de $\mathrm{O}_{\mathrm{NN}}$ en la región del nivel de impureza. Se puede apreciar que los oxígenos $\mathrm{O}_{\mathrm{NN} 1}$ y $\mathrm{O}_{\mathrm{NN} 3}$ (localizados en el plano $\left(\begin{array}{lll}1 & 1 & 0\end{array}\right)$ ) contribuyen mayoritariamente frente a los $\mathrm{O}_{\mathrm{NN} 2}$ del plano $\left(\begin{array}{lll}0 & 0 & 1\end{array}\right)$.

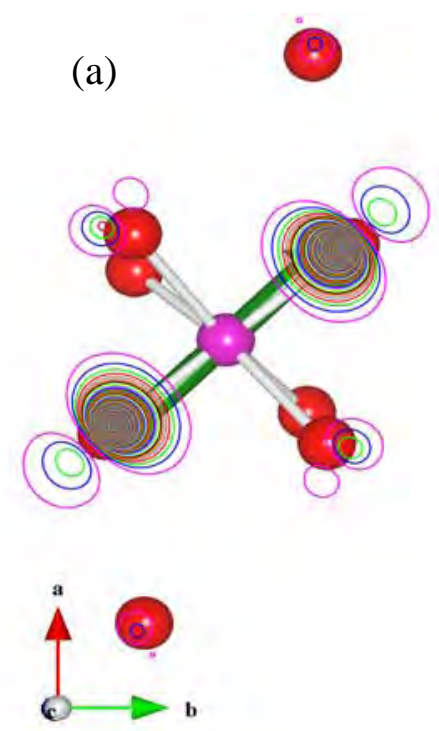

(b)
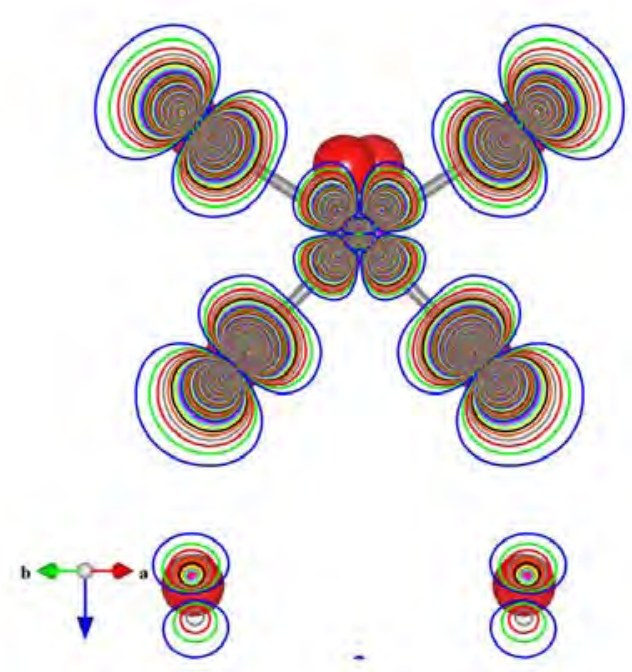

Figura 8.8: Curvas de nivel de la densidad de carga del electrón agregado a la celda neutra de $\mathrm{Y}_{2} \mathrm{O}_{3}$ :Cd con el Cd en el sitio C: (a) sobre el plano $\left(\begin{array}{lll}0 & 0 & 1\end{array}\right)$ que contiene los $\mathrm{O}_{\mathrm{NN} 2}$; (b) sobre el plano (1 110 ) que contiene la impureza Cd y sus vecinos $\mathrm{O}_{\mathrm{NN} 1}$ y $\mathrm{O}_{\mathrm{NN} 3}$. Con esferas rojas se representan los oxígenos, con esferas magenta el Cd y el vector verde apunta en la dirección [ $\left[\begin{array}{lll}1 & 1 & 0\end{array}\right]$ de $\mathrm{V}_{33}$ para el caso de celda cargada. El paso utilizado en las curvas de nivel es de $1 \%$ relativo al máximo. 


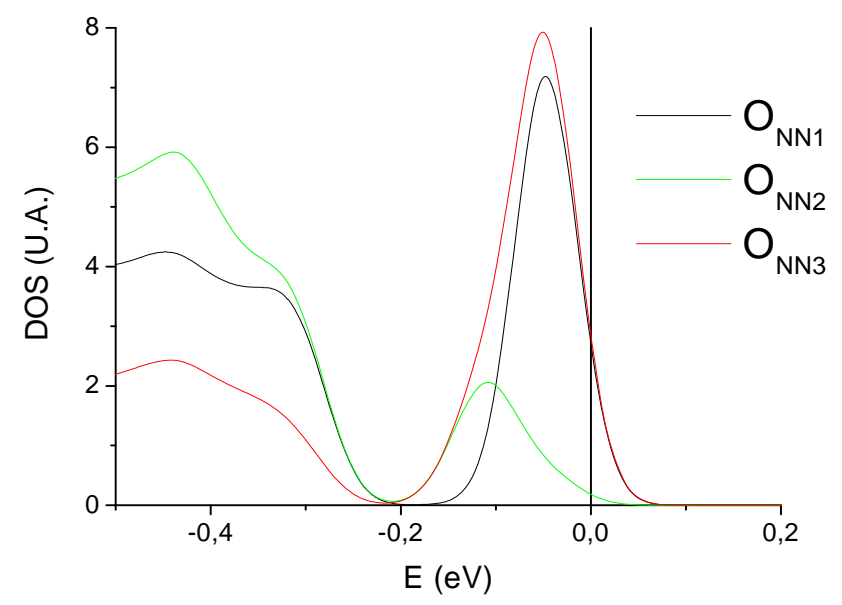

Figura 8.9: Contribuciones parciales a la densidad de estados en la región cercana al nivel de Fermi de los $\mathrm{O}_{\mathrm{NN}}$ de la impureza Cd localizada en el sitio $\mathrm{C}$ del $\mathrm{Y}_{2} \mathrm{O}_{3}$.

\section{8-5-3 Localización electrónica en el óxido $\mathrm{Sc}_{2} \mathrm{O}_{3}: \mathrm{Cd}$}

\section{Cd en sitio D:}

Ahora se analiza la localización del electrón agregado a la celda neutra del $\mathrm{Sc}_{2} \mathrm{O}_{3}$ para el caso del Cd localizado en el sitio D. Al igual que en las otras bixbitas, para proyectar las curvas de nivel de la densidad electrónica se eligieron el plano (1 -1 0) que contiene la impureza Cd y 2 de los 6 primeros vecinos oxígeno, y el plano (1 110 ) que contiene la impureza Cd y los 4 primeros vecinos $\mathrm{O}_{\mathrm{NN}}$ restantes (ver Figura 8.2). En la Figura 8.10 (a) se grafican las curvas de nivel de la densidad de carga proyectada sobre el plano (1 -1 0) y en la Figura 8.10 (b) las curvas de nivel de la densidad electrónica sobre el plano (1 10 ). Como en el caso de las otras bixbitas, la densidad electrónica presenta una mayor probabilidad la región de la impureza Cd y sus vecinos $\mathrm{O}_{\mathrm{NN}}$, mientras que se observa una débil probabilidad en las esferas de los $\mathrm{O}_{\mathrm{NNN}}$. Cabe destacar que la carga se distribuye homogéneamente en las seis ligaduras $\mathrm{Cd}-\mathrm{O}_{\mathrm{NN}}$ debido a que los seis primeros vecinos oxígeno del Cd localizado en el sitio D son equivalentes. Este hecho está en acuerdo con lo discutido en la sección 7-2-3 (c) donde se dedujo que el 70\% de los estados electrónicos del nivel de impureza se localizan en las esferas del Cd y sus vecinos $\mathrm{O}_{\mathrm{NN}}$. 
(a)

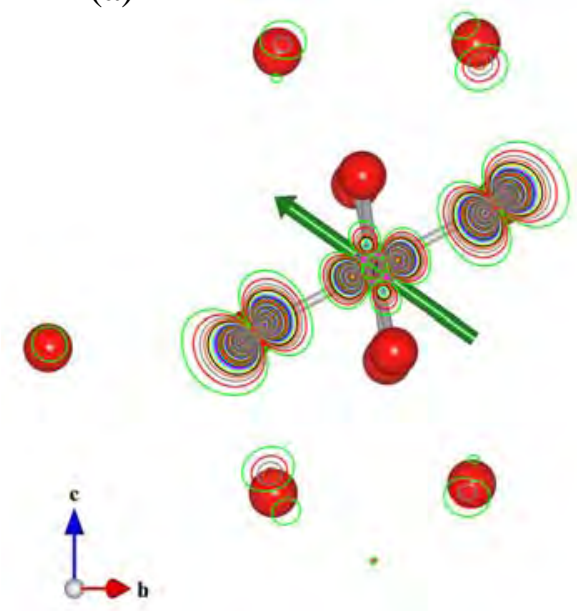

(b)

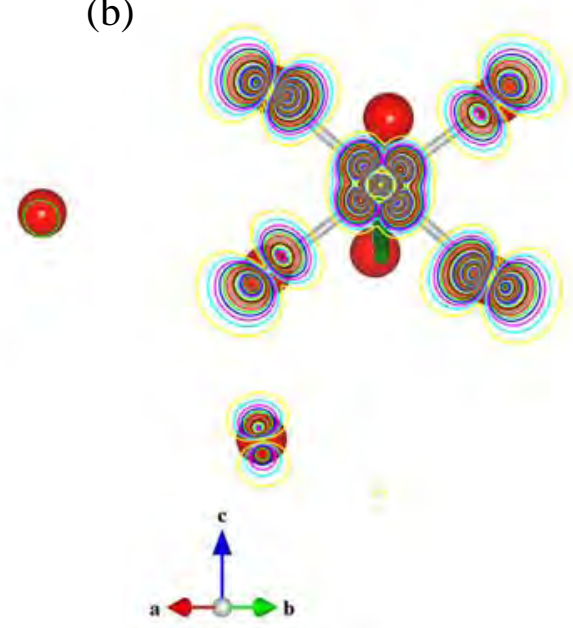

Figura 8.10: Curvas de nivel de la densidad de carga del electrón agregado a la celda neutra de $\mathrm{Sc}_{2} \mathrm{O}_{3}$ :Cd: (a) sobre el plano (1 -1 0); (b) sobre el plano (1 10 ). Con esferas rojas se representan los oxígenos, con esferas magenta el Cd y el vector verde apunta en la dirección [ [ 1111 de $\mathrm{V}_{33}$. El paso utilizado en las curvas de nivel es de $1 \%$ relativo al máximo.

\section{Cd en sitio C:}

En la Figura 8.11 (a) se grafica las curvas de nivel de la densidad de carga del electrón agregado a la celda neutra sobre el plano $\left(\begin{array}{lll}0 & 0 & 1\end{array}\right)$ que contiene los vecinos $\mathrm{O}_{\mathrm{NN} 2}$ de la impureza Cd situada en el sitio C. En la Figura 8.11 (b) se proyecta la densidad electrónica sobre el plano (1 110$)$ que contiene los otros cuatro primeros vecinos oxígeno $\mathrm{O}_{\mathrm{NN} 1}$ y $\mathrm{O}_{\mathrm{NN} 3}$.

$\mathrm{Al}$ igual que en las bixbitas analizadas anteriormente y en acuerdo a lo analizado en la sección 7-2-3 (c) (las esferas del Cd y sus vecinos $\mathrm{O}_{\mathrm{NN}}$ contribuyen con un 60 \% a los estados electrónicos del nivel de impureza), se aprecia que el electrón se localiza esencialmente sobre la impureza $\mathrm{Cd}$ y los átomos $\mathrm{O}_{\mathrm{NN}} \mathrm{y}$ se extiende hasta los segundos vecinos oxígeno. Además, se observa que la proyección de la densidad electrónica sobre el plano (1 110 ), que contiene a los oxígenos $\mathrm{O}_{\mathrm{NN} 1}$ y $\mathrm{O}_{\mathrm{NN} 3}$, posee mayor intensidad que la proyección sobre el plano $\left(\begin{array}{lll}0 & 0 & 1\end{array}\right)$ que contiene a los $\mathrm{O}_{\mathrm{NN} 2}$. Esto se puede interpretar a partir del gráfico de las densidades de estados de los primeros vecinos oxígeno en la región del nivel de impureza. En la Figura 8.12 se muestran las 3 DOS correspondientes a los 3 pares de vecinos $\mathrm{O}_{\mathrm{NN}}$. Claramente se observa que la contribución de los vecinos oxígeno $\mathrm{O}_{\mathrm{NN} 1} \mathrm{y}_{\mathrm{NN} 2}$ es dominante frente a los vecinos $\mathrm{O}_{\mathrm{NN} 3}$. 


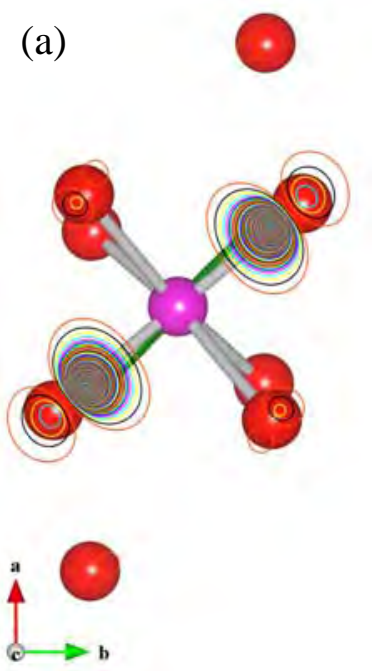

(b)

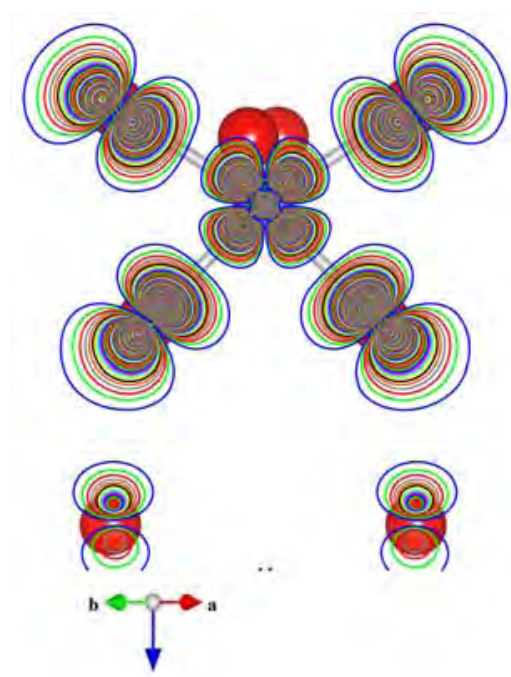

Figura 8.11: Curvas de nivel de la densidad de carga del electrón agregado a la celda neutra de $\mathrm{Sc}_{2} \mathrm{O}_{3}$ :Cd con el Cd en el sitio C: (a) sobre el plano $\left(\begin{array}{lll}0 & 0 & 1\end{array}\right)$ que contiene los oxígenos $\mathrm{O}_{\mathrm{NN} 2}$; (b) sobre el plano (1 110$)$ que contiene el Cd y los vecinos $\mathrm{O}_{\mathrm{NN} 1} \mathrm{y} \mathrm{O}_{\mathrm{NN} 3}$. Con esferas rojas se representan los oxígenos, con esferas magenta el Cd y el vector verde apunta en la dirección [ $\left[\begin{array}{lll}1 & 1 & 0\end{array}\right]$ de $\mathrm{V}_{33}$ para el caso de celda cargada. El paso utilizado en las curvas de nivel es de $1 \%$ relativo al máximo.

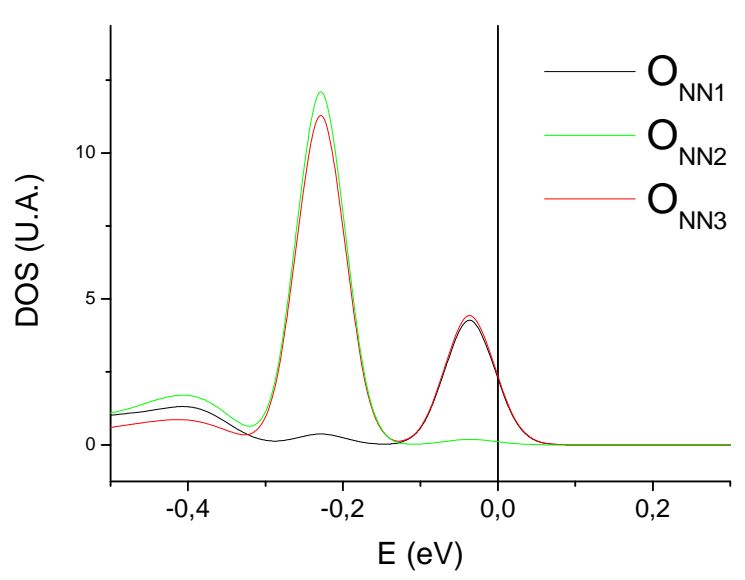

Figura 8.12: Contribución a la DOS en el entorno del nivel de Fermi de los $\mathrm{O}_{\mathrm{NN}}$ del Cd localizado en el sitio $\mathrm{C}$ del $\mathrm{Sc}_{2} \mathrm{O}_{3}$. 


\section{8-5-4 Localización electrónica en el óxido $\mathrm{SnO}_{2}: \mathrm{Cd}$}

En esta sección se analiza la localización de los dos electrones agregados a la celda neutra del rutilo $\mathrm{SnO}_{2}$. Para proyectar la densidad electrónica se escogieron los planos que contienen los vecinos $\mathrm{O}_{\mathrm{NN}}$ de la impureza Cd. En la Figura 8.13 (a) se grafica una de las caras de la supercelda del $\mathrm{SnO}_{2}$ :Cd paralela al plano (ll 0 l 1 ), la cual contiene cuatro átomos de Cd dispuestos en los vértices de la cara. El plano (lllll) contiene los vecinos $\mathrm{O}_{\mathrm{NN} 1}$ de la impureza $\mathrm{Cd}$, los cuales se disponen en el eje de la coordinación octaédrica del Cd. En la Figura 8.13 (b) se grafica el plano (1 110$)$ que pasa por los cuatro vecinos $\mathrm{O}_{\mathrm{NN} 2}$ que forman el plano octaédrico de la coordinación del Cd con sus primeros vecinos oxígenos.

En la Figura 8.14 (a) se grafican las curvas de nivel de la densidad electrónica (de los dos electrones) proyectadas sobre el plano (l 00 1) y en la Figura 8.14 (b) se detallan las curvas de nivel de la densidad sobre el plano (1 110$)$. En la proyección sobre el plano ( $\left.\begin{array}{lll}0 & 0 & 1\end{array}\right)$ se aprecia que la densidad electrónica es muy importante en el Cd y los primeros vecinos $\mathrm{O}_{\mathrm{NN} 1}$ dispuestos en la diagonal del octaedro, resulta apreciable en los segundos vecinos $\mathrm{O}_{\mathrm{NNN}} \mathrm{y}$ muy débil en el Sn. Por otro lado, la proyección sobre el plano $\left(\begin{array}{lll}1 & 1 & 0\end{array}\right)$ resulta muy importante sobre el $\mathrm{Cd}$ y los vecinos $\mathrm{O}_{\mathrm{NN} 2}$ que forman el plano basal del octaedro, pero es muy débil en los vecinos $\mathrm{Sn}_{\mathrm{NN}} \mathrm{y} \mathrm{O}_{\mathrm{NNN}}$. Por lo tanto los dos electrones agregados a la celda neutra del $\mathrm{SnO}_{2}$ : $\mathrm{Cd}$ se localizan esencialmente en el entorno del Cd y sus primeros vecinos $\mathrm{O}_{\mathrm{NN}}$ y con menor intensidad en los segundos vecinos $\mathrm{O}_{\mathrm{NNN}}$ que se sitúan en el eje del octaedro formado por el Cd y los $\mathrm{O}_{\mathrm{NN}}$ (ver Figura 8.14 (a)). Esto está en perfecto acuerdo con lo discutido en la sección 7-2-5 donde se dedujo que en las esferas del Cd y sus vecinos $\mathrm{O}_{\mathrm{NN}}$ se localizan un $70 \%$ de los estados electrónicos del nivel de impureza doblemente aceptor.

En la Figura 8.15 se grafican las contribuciones a la DOS de los primeros vecinos oxígeno del Cd y las PDOS de simetría $p$ de tales contribuciones para los tres estados de carga. Se puede apreciar que para el caso de los vecinos $\mathrm{O}_{\mathrm{NN} 1}$ que se encuentran en el eje del octaedro (formado por el $\mathrm{Cd}$ y sus $\mathrm{O}_{\mathrm{NN}}$ ) la contribución de las simetrías $p$ al nivel de impureza es esencialmente de carácter $p_{z}$. Por otro lado, la contribución de los vecinos $\mathrm{O}_{\mathrm{NN} 2}$ es esencialmente $p_{x} \mathrm{y} p_{y}$, mientras que $p_{z}$ se vuelve despreciable. 
(a)

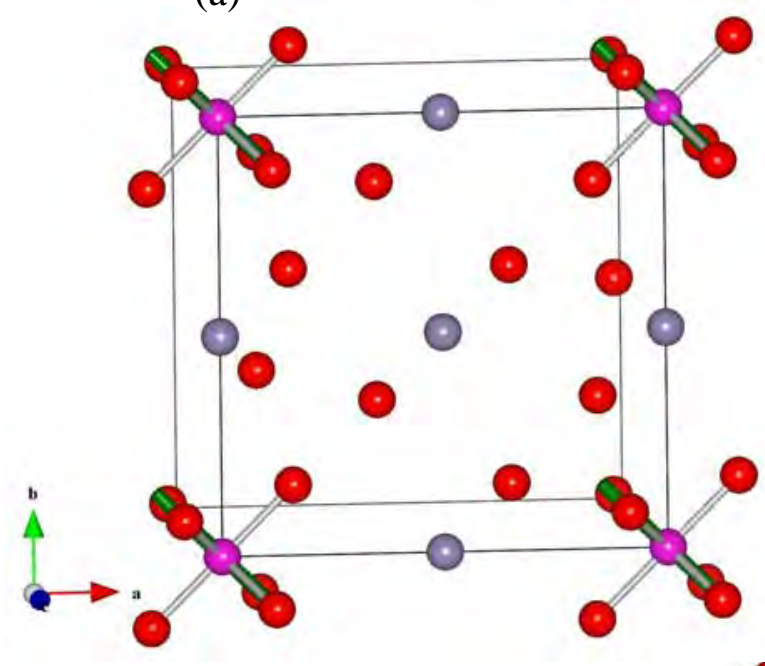

(b)

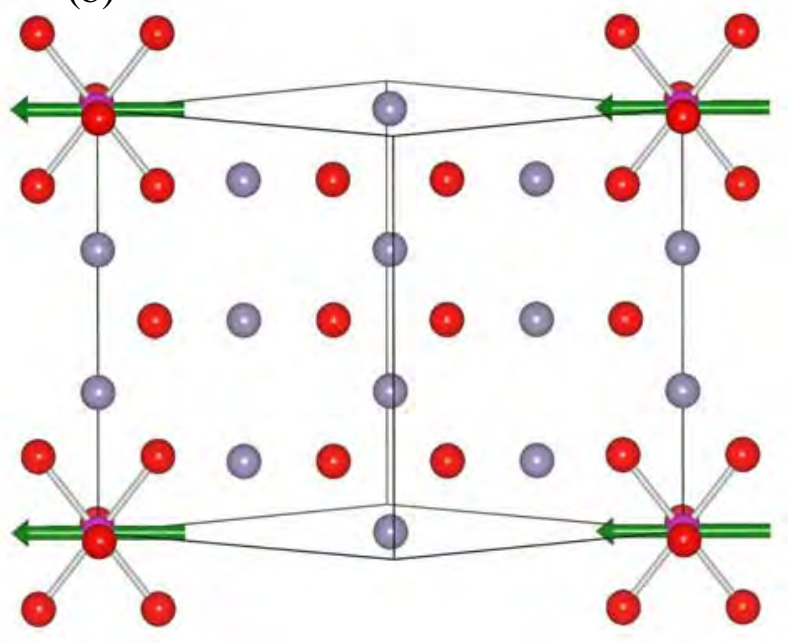

Figura 8.13: (a) Cara de la supercelda del $\mathrm{SnO}_{2}$ :Cd paralela al plano $\left(\begin{array}{lll}0 & 0 & 1\end{array}\right)$ con los $\mathrm{Cd}$ localizados en sus vértices. (b) Plano (1 10 ) de la celda. Con esferas magenta se representan las impurezas $\mathrm{Cd}$, con esferas rojas los oxígenos y con esferas grises los cationes. El vector verde simboliza la dirección [ 1 - 10 ] de la componente principal $V_{33}$.

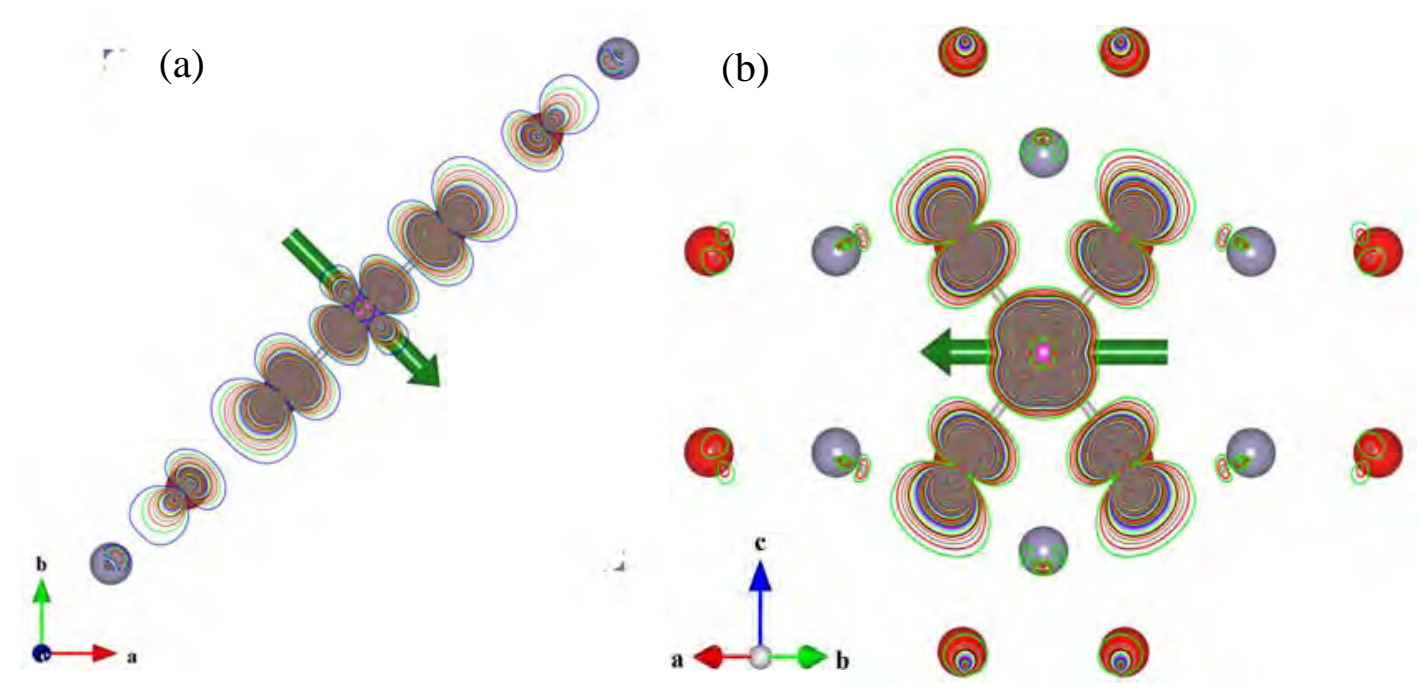

Figura 8.14: Curvas de nivel de la densidad de carga electrónica agregada a la celda neutra del $\mathrm{SnO}_{2}$ :Cd: (a) sobre el plano (lllll); (b) sobre el plano $\left(\begin{array}{lll}1 & 1 & 0\end{array}\right)$. Con esferas rojas se representan los oxígenos, con esferas grises los $\mathrm{Sn}$, con esferas magenta el Cd y en verde el vector en la dirección [ 1 - 1 0] de $\mathrm{V}_{33}$. El paso utilizado en las curvas de nivel es de 1\% relativo al máximo. 


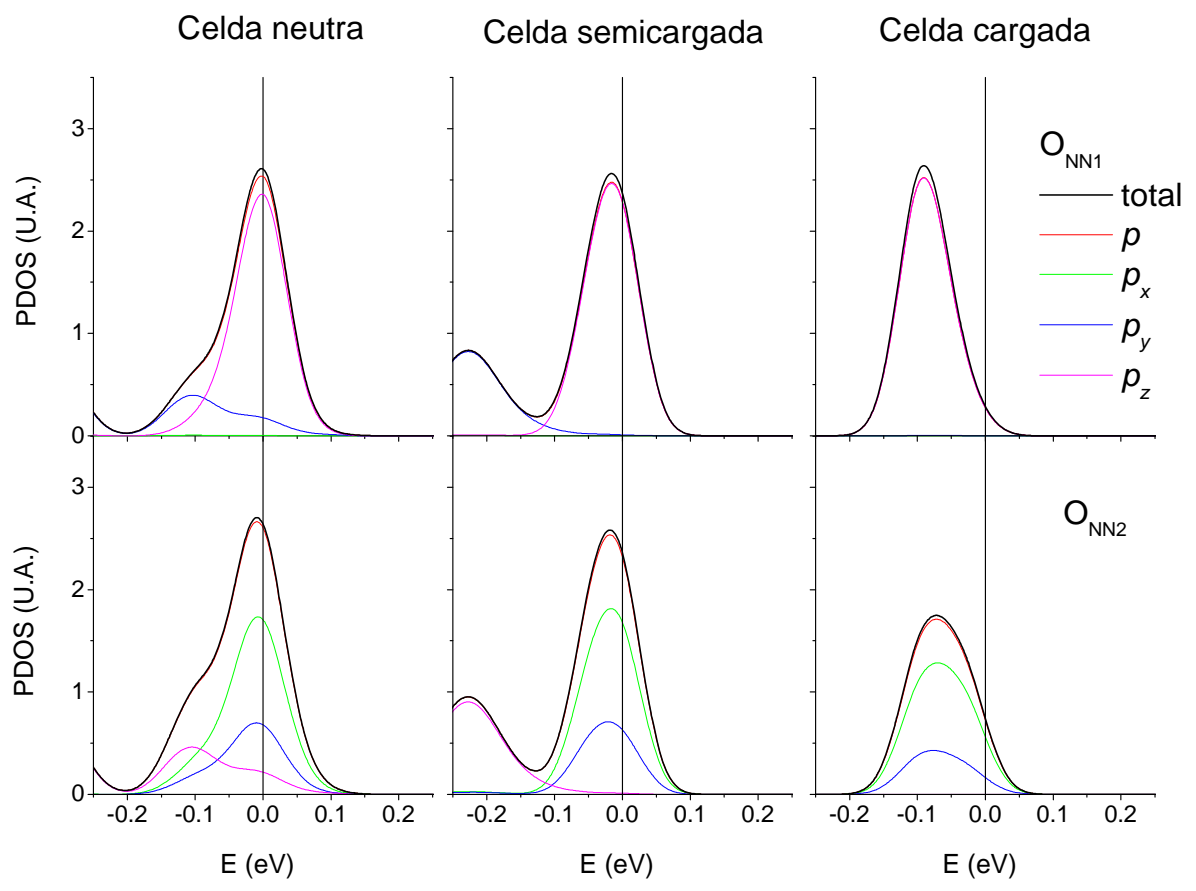

Figura 8.15: Contribuciones a la DOS de los $\mathrm{O}_{\mathrm{NN}}$ y PDOS de las simetrías $p$ de los $\mathrm{O}_{\mathrm{NN}}$ del $\mathrm{Cd}$ en el $\mathrm{SnO}_{2}$ graficadas en el entorno del nivel de Fermi.

Finalmente, en la Figura 8.16 se muestran las curvas de nivel de la densidad electrónica del primer (Fig. 8.16 (a) y (b)) y del segundo electrón (Fig. 8.16 (c) y (d)) agregados a la celda neutra. Desde la comparación de las proyecciones de la densidad electrónica se aprecia que el $1^{\text {o }}$ electrón agregado a la celda neutra presenta una mayor localización sobre el Cd y sus primeros vecinos $\mathrm{O}_{\mathrm{NN}}$. Además, se aprecia que la probabilidad de encontrar el $1^{\circ}$ y el $2^{\circ}$ electrón es muy importante tanto sobre los vecinos $\mathrm{O}_{\mathrm{NN} 1}$ del $\mathrm{Cd}$ ubicados sobre el plano $\left(\begin{array}{lll}0 & 0 & 1\end{array}\right)$ como sobre los primeros vecinos $\mathrm{O}_{\mathrm{NN} 2}$ ubicados sobre el plano (1 10 ). Por último, se concluye que el $1^{\circ}$ electrón agregado a la celda neutra del $\mathrm{SnO}_{2}$ es más localizado frente al $2^{\circ}$ electrón agregado el cual tiene un carácter más deslocalizado. 
(a)

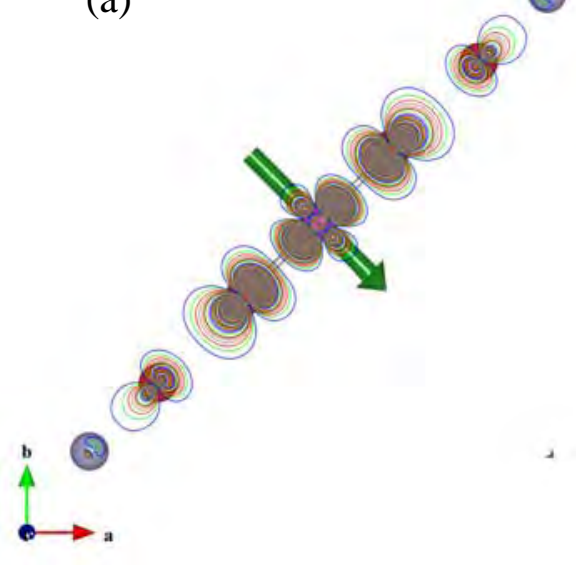

(c)

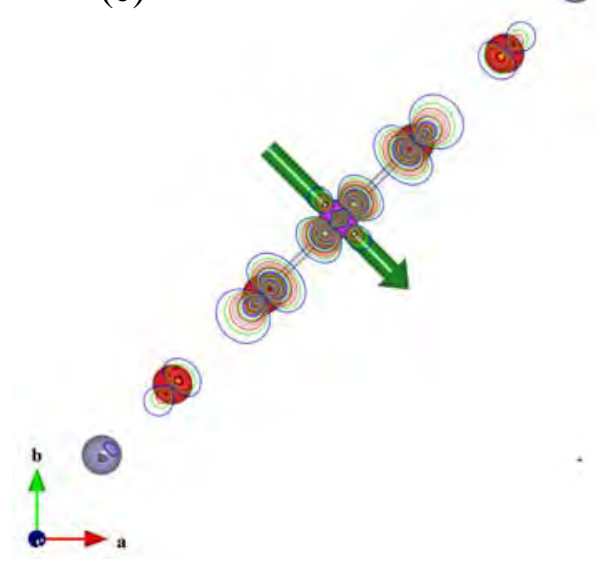

(b)
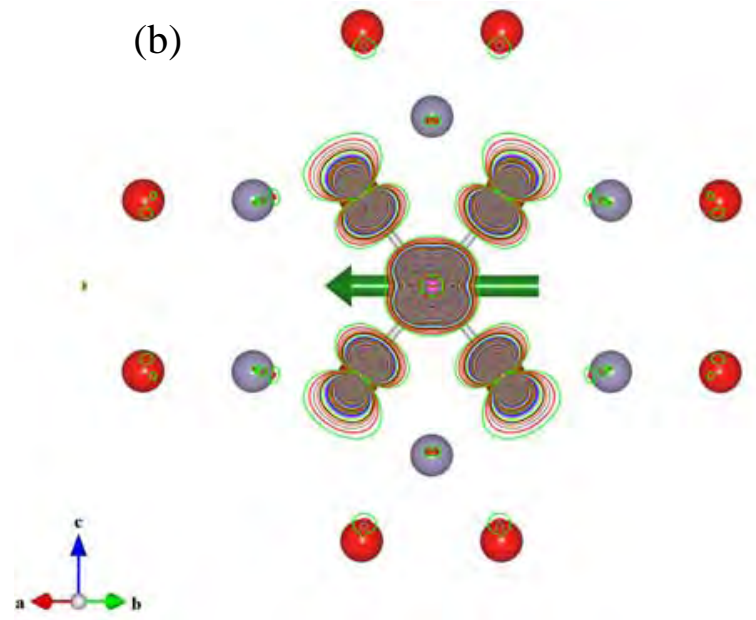

(d)
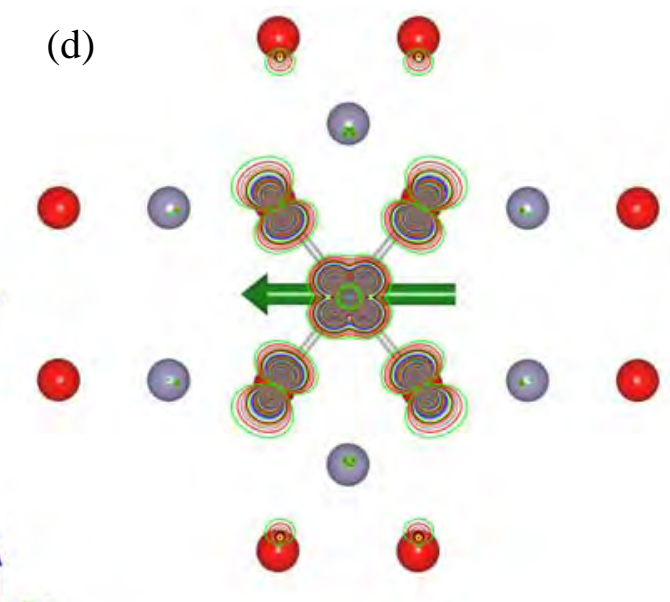

Figura 8.16: Curvas de nivel de la densidad de carga de cada electrón agregado a la celda neutra del $\mathrm{SnO}_{2}: \mathrm{Cd}$. Las proyecciones de la densidad de carga del $1^{\circ}$ electrón agregado se realizaron: (a) sobre el plano (0 0 1), (b) sobre el plano (1 1 $0)$. Las proyecciones del $2^{\circ}$ electrón agregado se realizaron: (c) sobre el plano (0 0 1), (d) sobre el plano (1 110 ). Con esferas rojas se representan los oxígenos, con esferas grises los $\mathrm{Sn}$ y con esferas magenta el Cd. El paso utilizado en las curvas de nivel es de $1 \%$ relativo al máximo.

\section{8-5-5 Localización electrónica en el ZnO:Cd}

En esta sección se analiza la localización del $1^{\circ}$ electrón removido y el agregado a la celda neutra del ZnO:Cd. Para proyectar la densidad electrónica se escogieron los planos ( $\left(\begin{array}{lll}0 & 1 & 0\end{array}\right)$ y $\left(\begin{array}{lll}0 & 0 & 1\end{array}\right)$. En la Figura 8.17 (a) se grafica una de las caras de la supercelda del ZnO:Cd paralela al plano ( $\left.\begin{array}{lll}0 & 1 & 0\end{array}\right)$, la cual contiene el vecino $\mathrm{O}_{\mathrm{NN} 2}$ de la impureza localizado en el eje $c$ por encima del Cd; mientras que en la Figura 8.17 (b) se

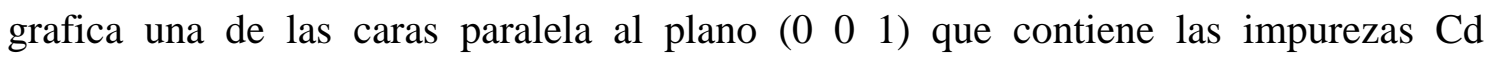


localizadas en los vértices de la supercelda y además, es paralela al plano que pasa por los tres primeros vecinos $\mathrm{O}_{\mathrm{NN} 1}$ del Cd.
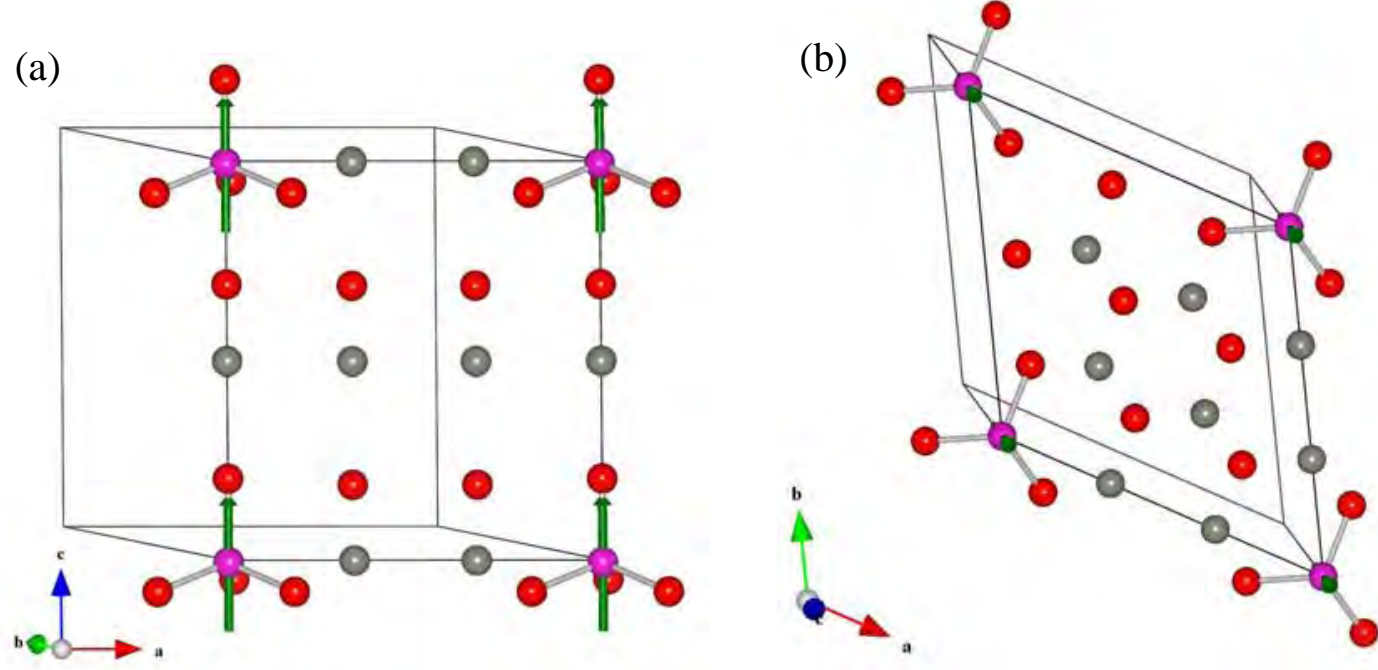

Figura 8.17: (a) Cara de la supercelda del ZnO:Cd paralela al plano $\left(\begin{array}{lll}0 & 1 & 0\end{array}\right)$ con los Cd localizados en sus vértices. (b) Plano $\left(\begin{array}{lll}0 & 0 & 1\end{array}\right)$ de la supercelda. Con esferas magenta se representan las impurezas $\mathrm{Cd}$, con esferas rojas los oxígenos y con esferas grises los cationes. El vector verde simboliza la dirección [ $\left[\begin{array}{lll}0 & 0 & 1\end{array}\right]$ de la componente principal $\mathrm{V}_{33}$.

En la Figura 8.18 se grafican las curvas de nivel de la densidad electrónica del electrón removido de la celda neutra sobre los diferentes planos atómicos. En la Figura 8.18 (a) se aprecia una débil localización del electrón sobre los cationes Zn y los O que están sobre la cara de la supercelda paralela al plano (l 10 ), en particular, sobre el $\mathrm{O}_{\mathrm{NN} 2}$ del Cd. mientras que la localización del electrón sobre las impurezas Cd es despreciable. En el plano ( $\left.\begin{array}{lll}0 & 0 & 1\end{array}\right)$ que contiene a los primeros vecinos $\mathrm{O}_{\mathrm{NN} 1}$ (Fig. 8.18 (b)) se observa una débil proyección de la densidad sobre los oxígenos.

En el plano ( $\left.\begin{array}{lll}0 & 0 & 1\end{array}\right)$ que contiene al Cd y los vecinos $\mathrm{Zn}_{\mathrm{NN}}$ de la impureza (Fig. 8.18 (c)), la densidad electrónica es débilmente apreciable en los Zn y se vuelve despreciable en la impureza. Finalmente en el plano (l $\left.\begin{array}{lll}0 & 1\end{array}\right)$ que contiene los vecinos $Z_{\mathrm{NNN}}$ y $\mathrm{O}_{\mathrm{NNN}}$ de la impureza (Fig. 8.18 (d) y (e), respectivamente) se aprecia que la proyección de la densidad electrónica es más débil que en el caso del plano $\left(\begin{array}{lll}0 & 0 & 1\end{array}\right)$ del Cd y los $\mathrm{Zn}_{\mathrm{NN}}$ (Fig. 8.18 (c)). Por lo tanto, se observa que el electrón removido de la supercelda neutra del ZnO:Cd está totalmente deslocalizado. 

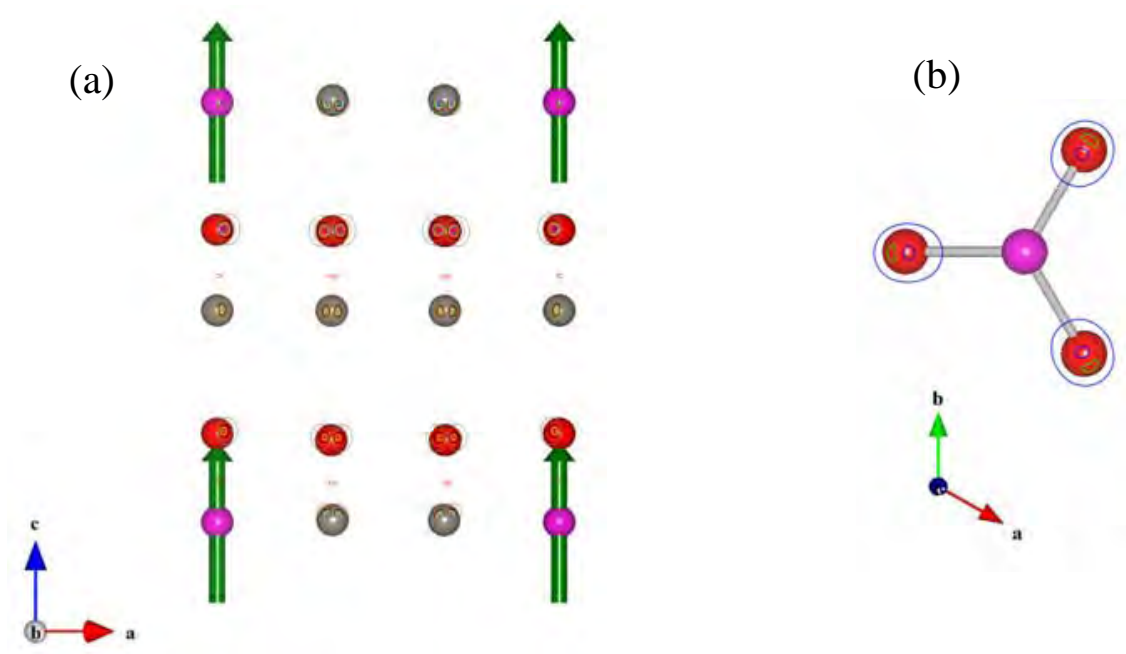

(c)

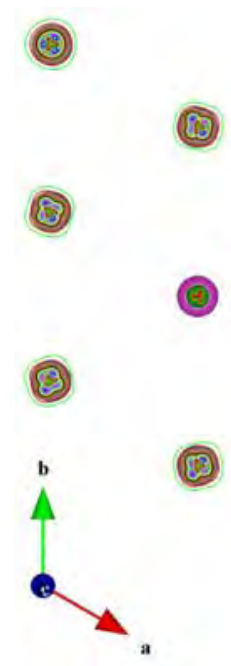

(2) (d)
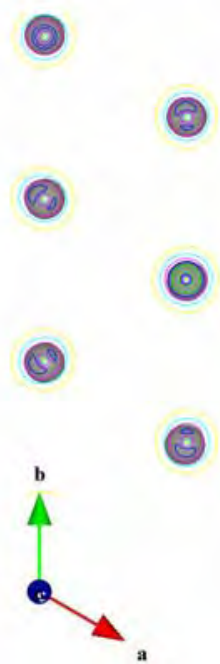

() (e)

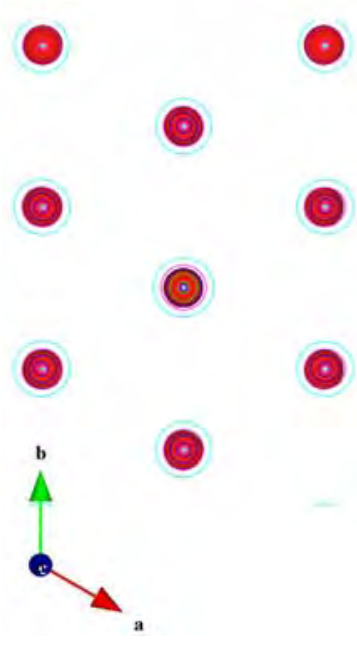

Figura 8.18: Curvas de nivel de la densidad de carga del electrón removido de la celda neutra del ZnO:Cd: (a) proyección de la densidad electrónica sobre el plano (0 1 0); (b) proyección sobre el plano $\left(\begin{array}{lll}0 & 0 & 1\end{array}\right)$ que contiene los primeros vecinos $\mathrm{O}_{\mathrm{NN}}$; (c) proyección sobre el plano ( $\left.\begin{array}{lll}0 & 0 & 1\end{array}\right)$ que contiene la impureza $\mathrm{Cd}$ y los primeros vecinos $\mathrm{Zn}_{\mathrm{NN}}$; (d) proyección sobre el plano (l $\left.\begin{array}{lll}0 & 0 & 1\end{array}\right)$ que contiene los segundos vecinos $Z_{n_{N N N}}$; (e) proyección sobre el plano (lllll) que contiene los vecinos $\mathrm{O}_{\mathrm{NNN}}$. Con esferas rojas se representan los oxígenos, con esferas grises los Zn, con esferas magenta la impureza Cd y con verde el vector en la dirección [ $\left[\begin{array}{lll}0 & 0 & 1\end{array}\right]$ de $\mathrm{V}_{33}$. El paso utilizado en las curvas de nivel es de 1\% relativo al máximo.

En la Figura 8.19 se grafican las curvas de nivel de la densidad electrónica correspondiente al electrón agregado a la celda neutra sobre los diferentes planos atómicos mostrados en la Figura 8.17. En la Figura 8.19 (a) se aprecia una débil localización sobre los átomos Zn y sobre las impurezas Cd que es más apreciable que

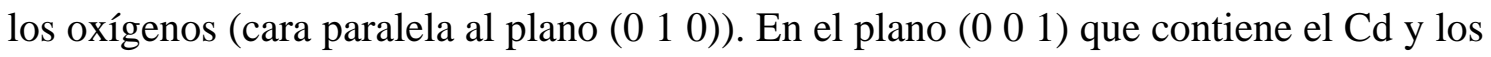
vecinos $Z_{\mathrm{NN}}$ de la impureza (Fig. 8.19 (c)), la densidad electrónica se vuelve despreciable. Finalmente en el plano $\left(\begin{array}{lll}0 & 0 & 1\end{array}\right)$ de los primeros y segundos vecinos oxígeno $\left(\mathrm{O}_{\mathrm{NN}}\right.$ y $\mathrm{O}_{\mathrm{NNN}}$, respectivamente) de la impureza Cd (Fig. 8.19 (b) y (e), 
respectivamente), se aprecia que la proyección de la densidad electrónica es más débil que en el caso del plano (l $\left.\begin{array}{lll}0 & 0 & 1\end{array}\right)$ de los $\mathrm{Zn}_{\mathrm{NNN}}$ (Fig. 8.19 (d)) en acuerdo con la proyección observada sobre el plano (0 10 ) (Fig. 8.19 (a)).

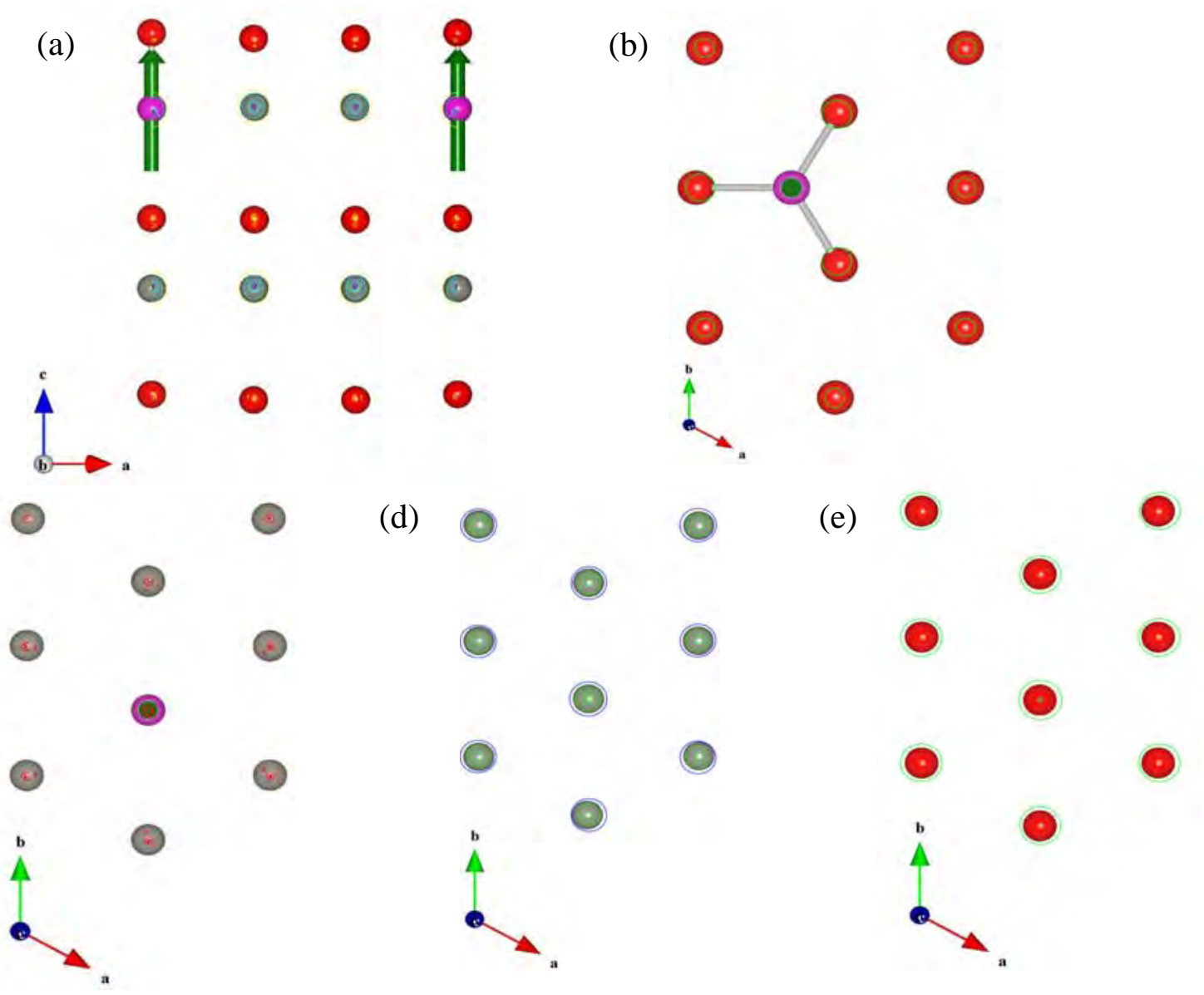

Figura 8.19: Curvas de nivel de la densidad de carga del electrón agregado a la celda neutra del ZnO:Cd: (a) proyección de la densidad electrónica sobre el plano (0 1 0); (b) proyección sobre el plano $\left(\begin{array}{lll}0 & 0 & 1\end{array}\right)$ que contiene los primeros vecinos $\mathrm{O}_{\mathrm{NN}}$; (c) proyección sobre el plano (l $\left.\begin{array}{lll}0 & 1\end{array}\right)$ que contiene la impureza Cd y los primeros vecinos $\mathrm{Zn}_{\mathrm{NN}}$; (d) proyección sobre el plano (l $\left.\begin{array}{lll}0 & 0 & 1\end{array}\right)$ que contiene los segundos vecinos $Z_{n_{N N N}}$; (e) proyección sobre el plano (0 0 1) que contiene los vecinos $\mathrm{O}_{\mathrm{NNN}}$. Con esferas rojas se representan los oxígenos, con esferas grises los $\mathrm{Zn}$ y con esferas magenta la impureza $\mathrm{Cd}$ y con verde el vector en la dirección [ $\left[\begin{array}{lll}0 & 0 & 1\end{array}\right]$ de $\mathrm{V}_{33}$. El paso utilizado en las curvas de nivel es de $1 \%$ relativo al máximo. 
En la Figura 8.20 se grafican las DOS del ZnO:Cd de los estados de carga descargado, neutro y cargado. Adicionalmente se representan las contribuciones de los átomos $\mathrm{Zn}$, de los átomos $\mathrm{O}$, del $\mathrm{Cd}$ y de sus primeros vecinos oxígeno. Se puede apreciar que al agregar un electrón a la celda descargada (Fig. 8.20 (a)) se llena la banda de valencia (Fig. 8.20 (b)) por lo que se infiere que tal electrón no contribuye a estados de impureza. Esto está en acuerdo con lo visto en la Figura 8.18 donde se dedujo que el electrón estaba totalmente deslocalizado. Por otro lado, al agregar un electrón a la celda neutra (Fig. 8.20 (b)) se puede observar que se ocupa parcialmente el pico de impureza apaisado introducido por el $\mathrm{Cd}$ en el fondo de la banda de conducción del $\mathrm{ZnO}$ (Fig. 8.20 (c)). Desde el análisis de la Fig. 8.19 se dedujo que la localización de electrón agregado a la celda neutra también se encuentra deslocalizado, esto se correlaciona con la forma del pico de impureza (apaisado) y con el hecho de que el Cd y sus primeros vecinos oxígeno prácticamente no aportan al nivel de impureza. De hecho, desde la contribución de las esferas al pico de impureza se obtuvo que los cationes Zn contribuyen con un $15 \%$, los átomos $\mathrm{O}$ con un 35\%, los vecinos $\mathrm{O}_{\mathrm{NN}}$ del Cd con un $9 \%$ y la impureza Cd con un $0.5 \%$.

(a)
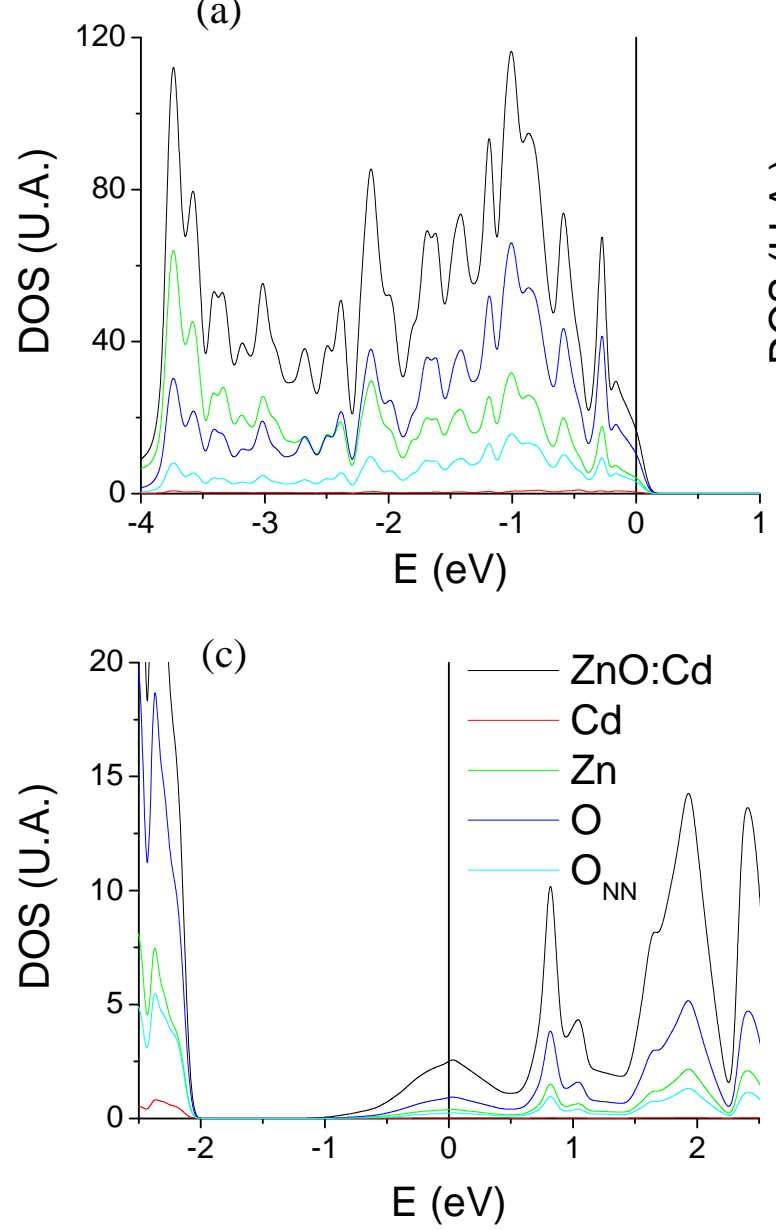

(b)

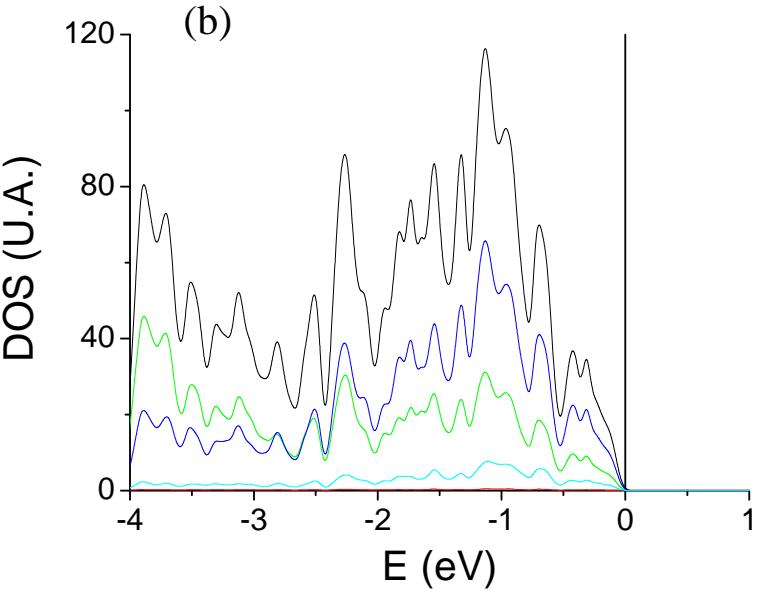

Figura 8.20: DOS del óxido $\mathrm{ZnO}$ dopado con Cd. Además se representan las contribuciones de las esferas atómicas de $\mathrm{Zn}, \mathrm{O}$, Cd y los vecinos $\mathrm{O}_{\mathrm{NN}}$. Se grafican las DOS correspondientes a los siguientes casos: (a) celda de $\mathrm{ZnO}$ :Cd descargada; (b) celda de $\mathrm{ZnO}: \mathrm{Cd}$ neutra; (c) celda ZnO:Cd cargada. 
Por lo tanto, se observa que el electrón agregado a la supercelda neutra del ZnO:Cd está totalmente deslocalizado lo cual se atribuye a la isovalencia que presenta el $\mathrm{Cd}^{2+}$ al reemplazar al catión nativo $\mathrm{Zn}^{2+}$ en la estructura del $\mathrm{ZnO}$. Además, este comportamiento deslocalizado se correlaciona con la forma apaisada de los picos de impureza introducidos por el Cd en el gap del ZnO (ver Fig.7.16). Esta correlación entre la deslocalización espacial de los estados electrónicos que aportan al nivel de impureza y la forma de dicho nivel también ha sido observado en $\mathrm{ZnO}$ dopado con impurezas Ta [Muñoz 2011b].

\section{8-5-6 Localización electrónica en el SnO:Cd}

En este apartado se analiza la localización electrónica para el caso de SnO:Cd, se estudian las proyecciones de la densidad de carga del electrón agregado y removido de la celda neutra. Para la proyección de la densidad electrónica en planos atómicos de la supercelda se escogieron los planos $\left(\begin{array}{lll}1 & 0 & 0\end{array}\right)$ y $\left(\begin{array}{lll}0 & 0 & 1\end{array}\right)$. En la Figura 8.21 (a) se grafica la

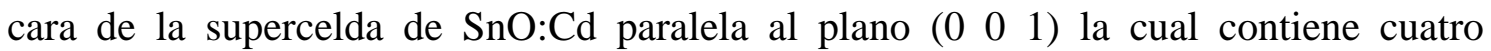
impurezas de Cd localizadas en sus vértices y en la Figura 8.21 (b) se grafica la cara paralela al plano $\left(\begin{array}{lll}0 & 0 & 1\end{array}\right)$.

(a)

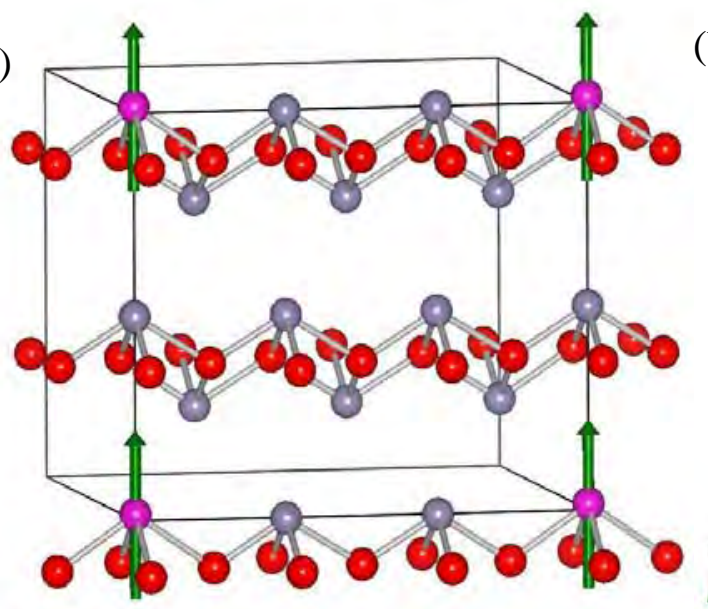

(b)

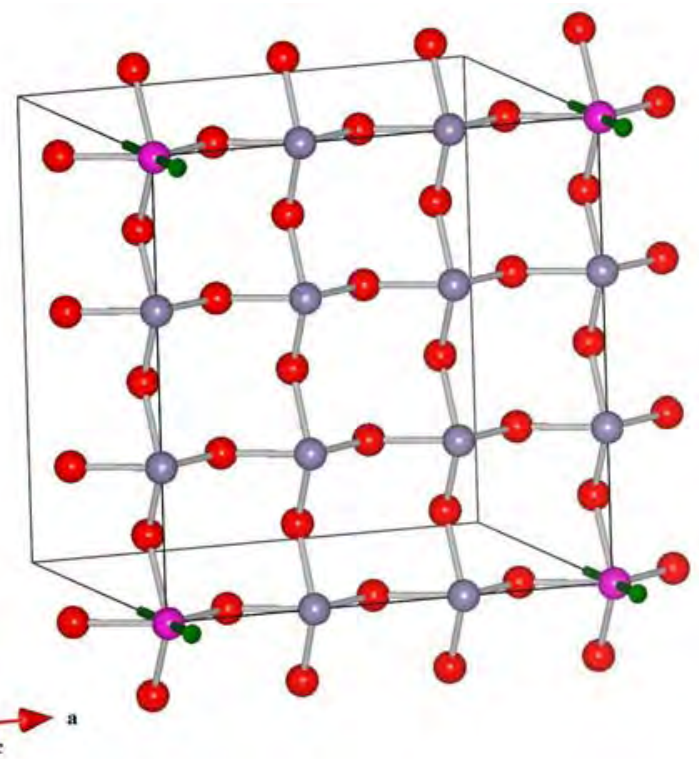

Figura 8.21: (a) Cara de la supercelda del SnO:Cd paralela al plano $\left(\begin{array}{lll}1 & 0 & 0\end{array}\right)$ con los Cd localizados en sus vértices. (b) Plano $\left(\begin{array}{lll}0 & 0 & 1\end{array}\right)$ de la supercelda. Con esferas magenta se representan las impurezas $\mathrm{Cd}$, con esferas rojas los oxígenos y con esferas grises los cationes. El vector verde simboliza la dirección [ $\left[\begin{array}{lll}0 & 0 & 1\end{array}\right]$ de la componente principal $\mathrm{V}_{33}$. 
En la Figura 8.19 se detallan las curvas de nivel de la densidad electrónica correspondiente al electrón agregado a la supercelda neutra del SnO:Cd sobre los planos (llll) y $\left.0 \begin{array}{lll}0 & 0 & 0\end{array}\right)$ de la celda. En el plano (l $\left.\begin{array}{lll}0 & 0\end{array}\right)$ que contiene la impureza (Fig. 8.22 (a)) se aprecia que la densidad está localizada parcialmente en la esfera del Cd. En cuanto al plano ( $\left.\begin{array}{lll}0 & 0 & 1\end{array}\right)$ que contiene los primeros vecinos oxígenos (Fig. 8.22 (b)) se observa que la densidad solo es apreciable en los cuatro $\mathrm{O}_{\mathrm{NN}}$. Desde la proyección de las curvas de nivel de la densidad sobre el plano (0 0 l 1 ) que contiene los segundos vecinos $\mathrm{O}_{\mathrm{NNN}}$ (Fig. 8.22 (c)) se aprecia una débil probabilidad de encontrar el electrón en dicho plano. Finalmente, desde las curvas de nivel proyectadas en el plano (1 00 ) que contiene al Cd y dos de sus primeros vecinos oxígeno (Fig. 8.22 (d)), se observa que la densidad electrónica es apreciable en el Cd y los primeros vecinos oxígenos. Por lo tanto, el electrón agregado a la celda neutra se encuentra parcialmente localizado en el Cd y los primeros vecinos $\mathrm{O}_{\mathrm{NN}}$; sin embargo se observa una apreciable deslocalización del electrón atribuida a la isovalencia de la impureza $\mathrm{Cd}^{2+}$ al reemplazar al ión nativo $\mathrm{Sn}^{2+}$ en la red del SnO. Cabe destacar que a diferencia del semiconductor $\mathrm{ZnO}$ donde el electrón agregado a la celda neutra está deslocalizado, el electrón agregado a la celda neutra del SnO (el cual presenta un carácter parcialmente localizado) se correlaciona con el gran nivel de impureza introducido por el Cd en el gap del SnO (ver Fig. 7.21).

(a)

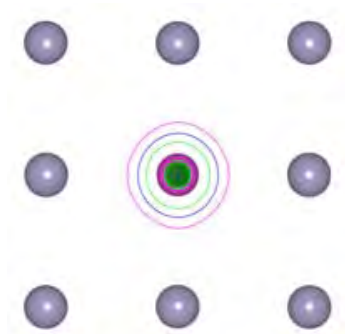

(d)
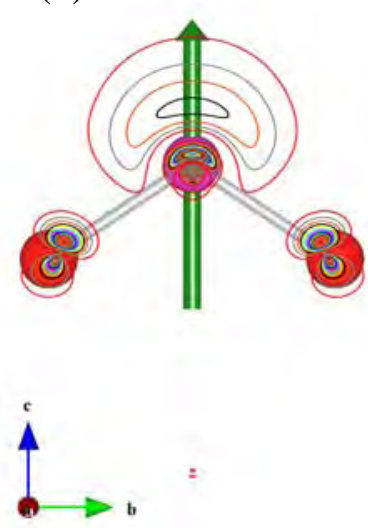

(b)
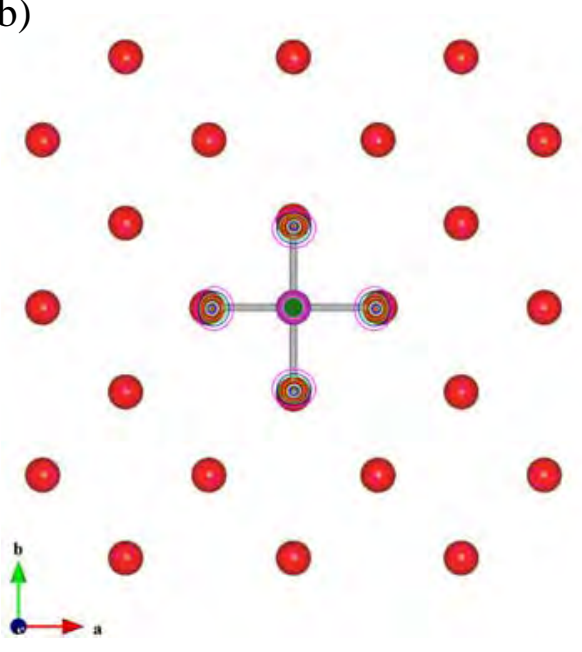
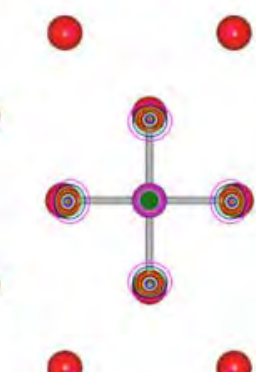

$+$
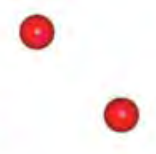

(c)

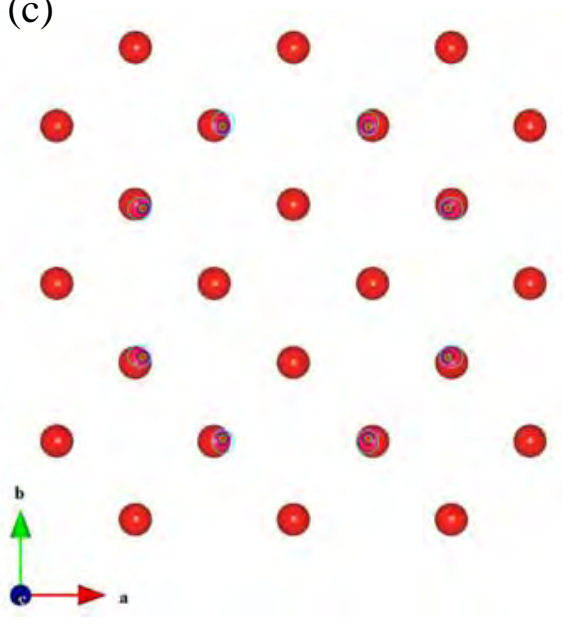

Figura 8.22: Curvas de nivel de la densidad de carga del electrón agregado a la celda neutra del SnO:Cd: (a) proyección de la densidad electrónica sobre el plano (l 0 l 1) que contiene al Cd; (b) proyección sobre el plano (0 $\left.\begin{array}{l}0 \\ 0\end{array}\right)$ que contiene los vecinos $\mathrm{O}_{\mathrm{NN}}$; (c) proyección sobre el plano $\left(\begin{array}{lll}0 & 0 & 1\end{array}\right)$ que contiene los vecinos $\mathrm{O}_{\mathrm{NNN}}$; (d) proyección sobre el plano $\left(\begin{array}{lll}1 & 0 & 0\end{array}\right)$ que contiene al Cd y dos oxígenos $\mathrm{O}_{\mathrm{NN}}$. Con esferas rojas se representan los oxígenos, con esferas grises los Sn y con esferas magenta la impureza Cd. El paso utilizado en las curvas de nivel es de 1\% relativo al máximo. 
En la Figura 8.23 se grafican las curvas de nivel de la densidad electrónica del electrón removido de la celda neutra sobre los planos $\left(\begin{array}{lll}0 & 0 & 1\end{array}\right)$ y $\left(\begin{array}{lll}0 & 1 & 0\end{array}\right)$. En la Fig. 8.23 (a) se aprecia que el electrón está débilmente localizado sobre los oxígenos, excepto sobre los $\mathrm{O}_{\mathrm{NN}}$, que se encuentran en el plano (l $\left.\begin{array}{lll}0 & 0 & 1\end{array}\right)$. En las Figuras 8.23 (b) y (c) también se observa una débil localización sobre los oxígenos $\mathrm{O}_{\mathrm{NNN}}$ que se encuentran en el plano ( $\left(\begin{array}{lll}0 & 0 & 1\end{array}\right)$ y sobre el $\mathrm{Cd}$ y dos de sus vecinos $\mathrm{O}_{\mathrm{NN}}$ en el plano $\left(\begin{array}{lll}0 & 1 & 0\end{array}\right)$, respectivamente. La evidente deslocalización del electrón removido de la celda neutra está en acuerdo con su pertenencia a la banda de valencia del semiconductor, es decir que no pertenece al pico de impureza. Cabe remarcar que a pesar de que electrón removido de la celda neutra presenta un carácter deslocalizado, es capaz de perturbar el entorno de la impureza debido a que la componente principal $\mathrm{V}_{33}$ del GCE observado por la sonda-impureza ${ }^{111} \mathrm{Cd}$ cambia su magnitud en un $50 \%$.
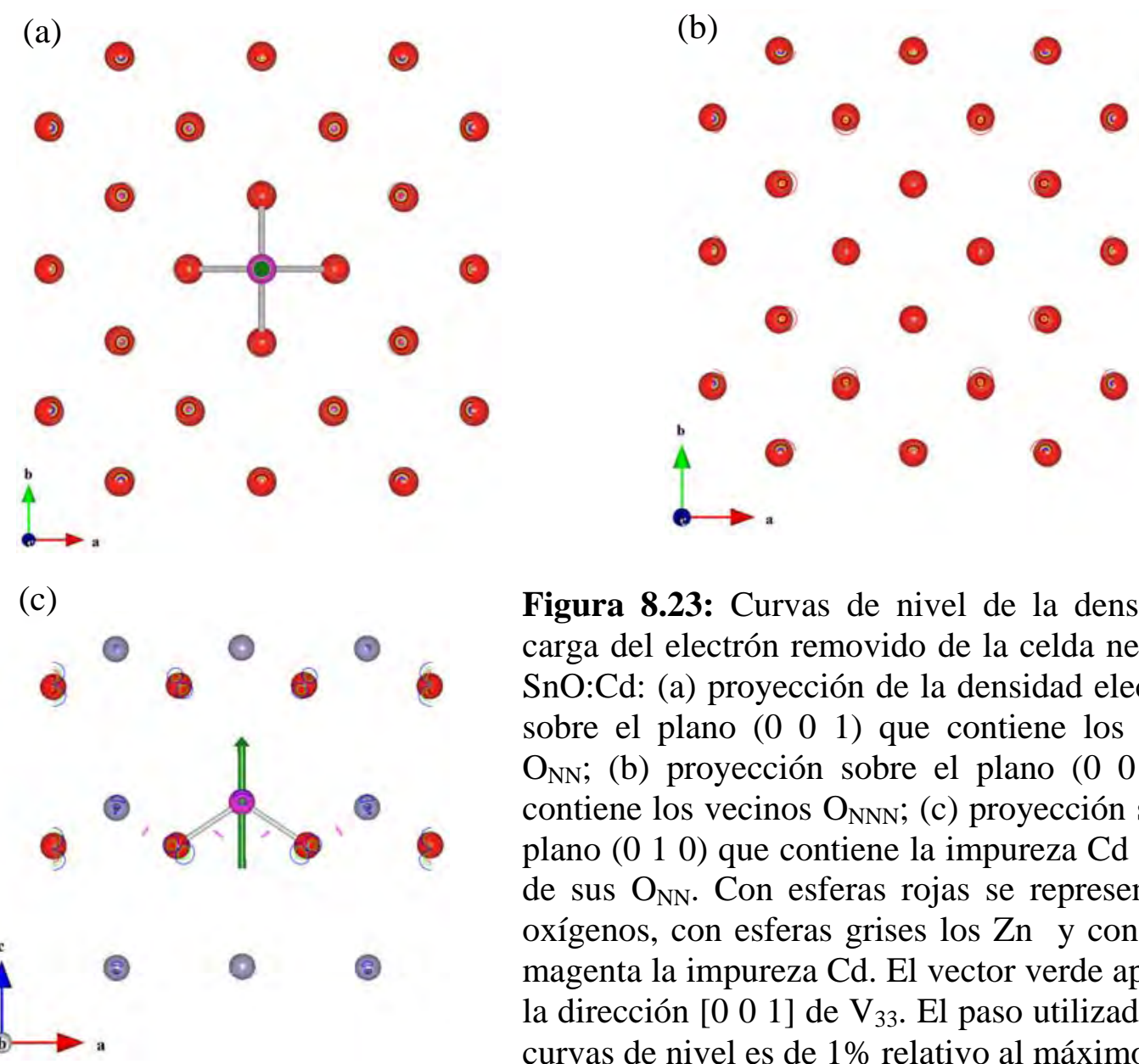

Figura 8.23: Curvas de nivel de la densidad de carga del electrón removido de la celda neutra del SnO:Cd: (a) proyección de la densidad electrónica sobre el plano $\left(\begin{array}{lll}0 & 0 & 1\end{array}\right)$ que contiene los vecinos $\mathrm{O}_{\mathrm{NN}}$; (b) proyección sobre el plano $\left(\begin{array}{lll}0 & 0 & 1\end{array}\right)$ que contiene los vecinos $\mathrm{O}_{\mathrm{NNN}}$; (c) proyección sobre el plano $\left(\begin{array}{lll}0 & 1 & 0\end{array}\right)$ que contiene la impureza Cd con dos de sus $\mathrm{O}_{\mathrm{NN}}$. Con esferas rojas se representan los oxígenos, con esferas grises los $\mathrm{Zn}$ y con esferas magenta la impureza $C d$. El vector verde apunta en la dirección [ll $\left.\begin{array}{lll}0 & 0 & 1\end{array}\right]$ de $\mathrm{V}_{33}$. El paso utilizado en las curvas de nivel es de $1 \%$ relativo al máximo. 
En la Figura 8.24 se grafican las DOS del semiconductor SnO:Cd junto con las contribuciones de las esferas de los átomos $\mathrm{Sn}$, de los $\mathrm{O}$, de la impureza Cd y de sus primeros vecinos oxígeno. Al agregar un electrón a la celda descargada (Fig. 8.24 (a)), se observa que se llena el nivel de impureza del tope de la banda de valencia. Mientras que al agregar un electrón a la celda neutra (Fig. 8.24 (b)) se ocupa parcialmente el nivel de impureza del fondo de la banda de conducción (Fig. 8.24 (c)). Desde la integración sobre las esferas atómicas de los estados electrónicos que aportan al nivel de impureza del tope de la banda de valencia se tiene que los átomos O contribuyen con un 37\%, los cationes Sn con un $27 \%$, la impureza $\mathrm{Cd}$ con $1 \%$ y sus vecinos $\mathrm{O}_{\mathrm{NN}}$ con un $3 \%$ por lo que la localización en el entorno del Cd y sus primeros vecinos oxígeno es despreciable. Desde la integración de los estados electrónicos del nivel que se encuentra en el fondo de la banda de conducción se tiene que los cationes Sn contribuyen con un 31\%, los átomos $\mathrm{O}$ con un $24 \%$, la impureza Cd con un $10 \%$ y sus vecinos $\mathrm{O}_{\mathrm{NN}}$ también con un $10 \%$. Por lo tanto, el electrón agregado a la celda neutra presenta un apreciable localización sobre el Cd y sus vecinos $\mathrm{O}_{\mathrm{NN}}$.
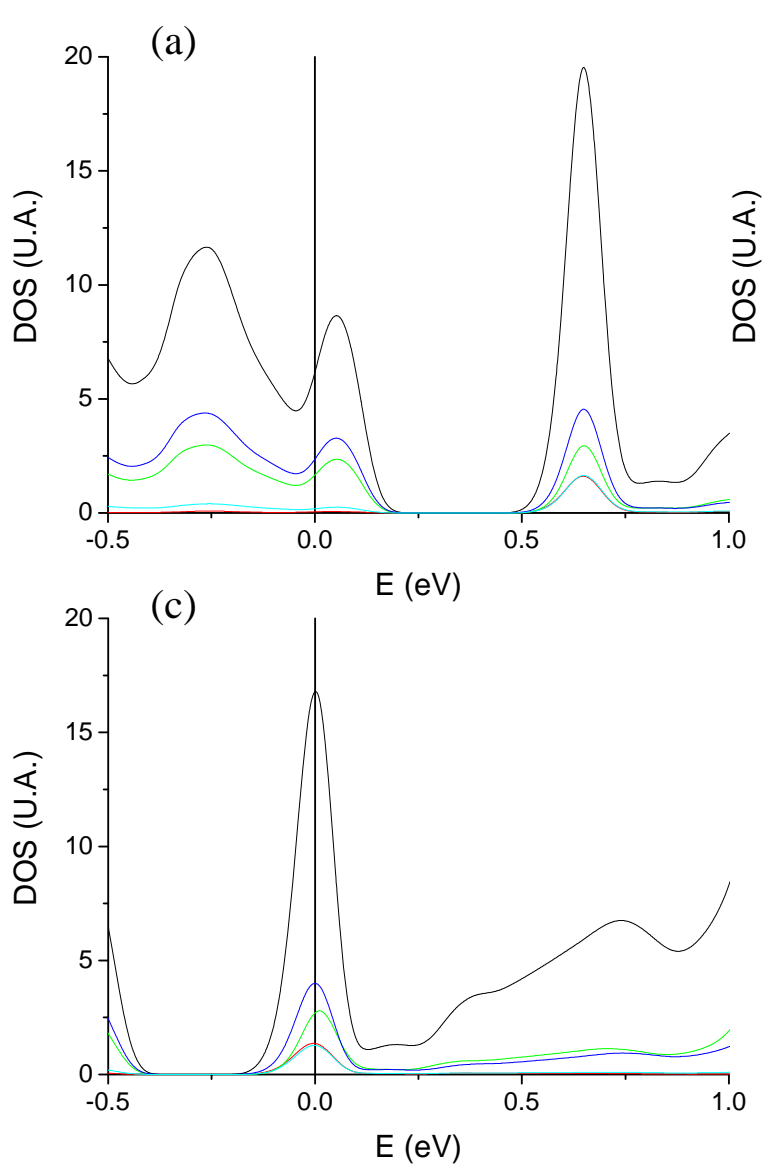

(b)

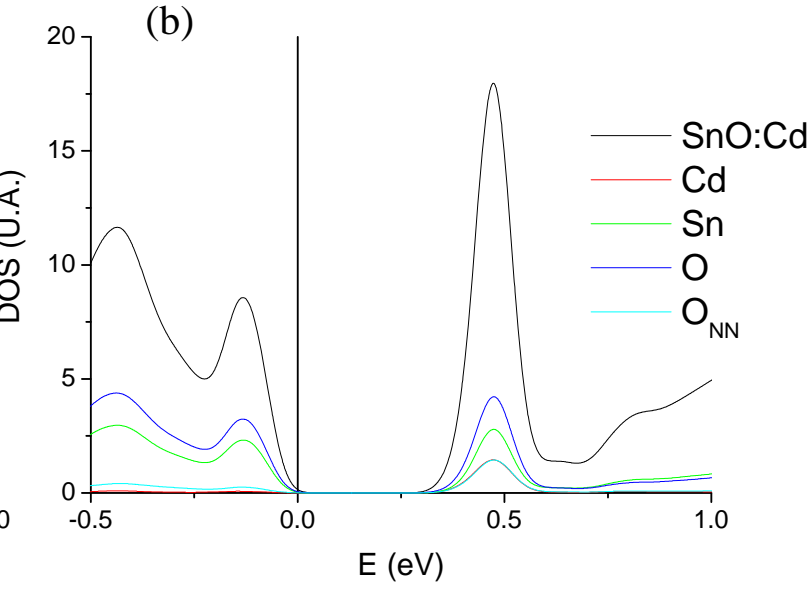

Figura 8.24: DOS del óxido $\mathrm{SnO}$ dopado con Cd. Además se representan las contribuciones de las esferas atómicas de $\mathrm{Sn}, \mathrm{O}$, Cd y los vecinos $\mathrm{O}_{\mathrm{NN}}$. Se grafican las DOS correspondientes a los siguientes casos: (a) celda descargada; (b) celda neutra; (c) celda cargada. 


\section{8-6 Modelo semi-empírico - ab initio de interacciones hiperfinas dinámicas}

En este apartado se desarrolla el modelo de interacciones dinámicas basado en medidas PAC con la sonda $\left({ }^{111} \mathrm{In} \rightarrow\right){ }^{111} \mathrm{Cd}$ y cálculos APW+lo del GCE en el sitio de la sonda-impureza Cd en función del estado de carga de la supercelda e los óxidos estudiados en este trabajo. Tales interacciones hiperfinas son debidas al proceso de after-effects, el cual ocurre luego del decaimiento por EC del isótopo ${ }^{111} \mathrm{In}\left((\mathrm{EC}) \rightarrow{ }^{111} \mathrm{Cd}\right)$, y dependiendo de las características de la disponibilidad y movilidad electrónica del óxido dopado bajo estudio origina un tensor GCE dependiente del tiempo en el sitio de la sonda,.

Como se describió en el Capítulo 3, en la presente tesis el ajuste de los espectros PAC fue realizado con un factor de perturbación basado en el modelo de Bäverstam y Othaz [Bäverstam 1972], el cual está dado por el producto de dos factores, uno estático y otro dinámico, como se muestra en la ecuación 8.1.

$$
G_{22}(t)=G_{22}^{s}(t) \cdot G_{22}^{d}(t)
$$

El factor estático toma la forma usual (ver ecuación 2.26), mientras que el factor dinámico depende de dos parámetros $\left(\lambda_{\mathrm{r}} \mathrm{y} \lambda_{\mathrm{g}}\right)$ y tiene la forma:

$$
G_{22}^{d}(t)=\frac{\lambda_{g}}{\lambda_{g}+\lambda_{r}}+\frac{\lambda_{r}}{\lambda_{g}+\lambda_{r}} e^{-\left(\lambda_{g}+\lambda_{r}\right) t}
$$

En este modelo, como se mencionó en el Capítulo 3, el parámetro dinámico $\lambda_{\mathrm{r}}$ se denomina constante de relajación de Abragam y Pound y se relaciona con la intensidad de la interacción dinámica atribuida al AE; en el caso de la constante de recuperación $\lambda_{\mathrm{g}}$, su inverso $\left(\tau_{\mathrm{g}}\right)$ se puede interpretar como la vida media de los huecos electrónicos en el entorno de la sonda-impureza ${ }^{111} \mathrm{Cd}$ y dependiendo si alcanza (o no) la ventana temporal del estado intermedio de la cascada $\gamma-\gamma$ del ${ }^{111} \mathrm{Cd}$, la sonda PAC “observará” una interacción dinámica (o estática). 


\section{8-6-1 Modelo semi-empírico - ab initio aplicado al caso de los óxidos con estructura bixbita}

Desde el punto de vista experimental, en los óxidos con estructura bixbita se observó, en general, que el sitio C presenta mayor atenuación dinámica que el sitio D en el rango de temperaturas donde la misma está presente excepto para el caso de $\mathrm{Y}_{2} \mathrm{O}_{3}$ donde la tendencia se extiende a todo el rango de temperaturas. Esto se correlaciona con el hecho que el parámetro $\lambda_{\mathrm{r}}$ del sitio $\mathrm{C}$ está por encima que el del sitio $\mathrm{D}$ en presencia de relajación dinámica. En cambio el parámetro $\lambda_{\mathrm{g}}$ tiene comportamientos dispares en las bixbitas: en el óxido $\operatorname{In}_{2} \mathrm{O}_{3}, \lambda_{\mathrm{g}}$ del sitio $\mathrm{C}$ es mayor que en el sitio D cuando el proceso dinámico es más intenso, pero en los otros dos sesquióxidos $\left(\mathrm{Sc}_{2} \mathrm{O}_{3}\right.$ e $\left.\mathrm{Y}_{2} \mathrm{O}_{3}\right)$ el parámetro $\lambda_{\mathrm{g}}$ es similar para ambos sitios en presencia de atenuación dinámica.

Por otro lado, a partir del estudio APW+lo de la dependencia del GCE calculado en el sitio de la impureza como función del estado de carga de la impureza se determinó que el GCE del sitio C es fuertemente dependiente del estado de carga mientras que el del sitio D no presenta dependencia alguna. Cabe destacar que estructuralmente el sitio D tiene simetría axial mientras que el sitio C es altamente asimétrico. Además, a partir del análisis de los gráficos de densidades de estados parciales (PDOS) de las simetrías $d$ del Cd que contribuyen al nivel de impureza, se encontró que para el caso de Cd en sitio D todas las simetrías $d$, excepto la simetría $d_{z^{2}}$, aportan uniformemente al nivel de impureza. De este modo, al llenar o vaciar el nivel según sea agregando o sacando un electrón al sistema dopado, el GCE en el sitio D no presenta cambios. Mientras que para el caso de Cd en sitio C, al nivel de impureza solo aportan las simetrías $d_{x z}$ y $d_{y z}$ por lo que al llenar o vaciar el nivel modificando el número de electrones del sistema, el GCE cambia en magnitud, signo y dirección.

Por lo tanto, a partir de la comparación de las medidas PAC y los resultados de los cálculos APW+lo para el caso de Cd en el sitio C y se concluye que altas temperaturas se “apaga” el proceso dinámico debido a que el electrón ocupa el hueco electrónico en un tiempo suficientemente rápido como para no alcanzar la ventana temporal de la medida y la localización de dicho electrón alcanza esencialmente el entorno del Cd y sus vecinos $\mathrm{O}_{\mathrm{NN}}$ y se extiende levemente a los $\mathrm{O}_{\mathrm{NNN}}$ (ver Figuras 8.5, 8.8 y 8.11). Cabe destacar que este análisis está avalado por la dependencia de los parámetros $\lambda_{\mathrm{g}} \mathrm{y} \lambda_{\mathrm{r}}$ con la temperatura. Por último, el GCE observado a altas temperaturas, esto es donde el 
proceso dinámico se extingue, corresponde al estado de carga de la impureza cargado (con el electrón agregado a la celda neutra en el entorno del Cd y sus vecinos $\mathrm{O}_{\mathrm{NN}}$ ).

En el caso de Cd en sitio D, es posible caracterizar el origen de la ausencia de la interacción dinámica la cual podría deberse a tres factores: uno de ellos es que la vida media de los huecos electrónicos sea suficientemente pequeña como para no alcanzar la ventana temporal de la medida; el otro factor es que los huecos electrónicos sean muy deslocalizados de modo que los cambios en la densidad electrónica de carga no son observados por la sonda-impureza; y el tercer factor se relaciona con la simetría del sitio, en el caso que el sitio presente una alta simetría el GCE observado en tal sitio será independiente de los cambios en la densidad de carga al no cambiar la simetría puntual de la sonda Cd. Para el caso de la sonda-impureza Cd localizada en el sitio D de la estructura bixbita, el primer argumento no es aplicable porque los parámetros $\lambda_{\mathrm{g}}$ ajustados en el sitio D son comparables a los ajustados en el sitio $C$ del $\operatorname{In}_{2} \mathrm{O}_{3}$ y similares a los $\lambda_{\mathrm{g}}$ ajustados en el sitio $\mathrm{C}$ de las bixbitas $\mathrm{Sc}_{2} \mathrm{O}_{3}$ e $\mathrm{Y}_{2} \mathrm{O}_{3}$. Mientras que el segundo factor deja de ser aplicable a este caso debido a que en la sección 7-2-3 del capítulo 7 y en la sección anterior se observó que el electrón agregado y removido de la celda neutra de las bixbitas (para el caso de Cd en sitio D) se localizan esencialmente en el entorno de la impureza y sus primeros vecinos oxígenos (ver Figuras 8.4, 8.7 y 8.10). Por todo esto la ausencia de atenuación dinámica en las medidas de ${ }^{111} \mathrm{Cd}$ localizado en sitio D se atribuye a la alta simetría que posee tal sitio, lo cual se traduce en la simetría observada en las PDOS con carácter $d$ en la esfera del Cd.

Finalmente, para el caso de las bixbitas el doble abordaje experimental y de primeros principios permite correlacionar la fuerte (débil) dependencia del GCE con el estado de carga calculado en el sitio C (D) con un alto (bajo) valor del parámetro $\lambda_{\mathrm{r}}$ medido en el sitio C (D). Además, cabe mencionar que en el caso de los óxidos con estructura bixbita, como era de esperar no existe correlación alguna entre la constante de recuperación $\lambda_{\mathrm{g}} \mathrm{y}$ la dependencia funcional del GCE con el estado de carga.

\section{8-6-2 Modelo semi-empírico - ab initio aplicado al óxido $\mathrm{SnO}_{2}$}

Ahora se aborda el caso del dióxido de estaño $\left(\mathrm{SnO}_{2}\right)$. Desde el estudio PAC se observa que fueron necesarias dos interacciones hiperfinas (HFI1 y 2) para ajustar las medidas PAC en todo el rango de temperaturas. La interacción HFI1 resultó dinámica por debajo de los $600 \mathrm{~K}$ mientras que HFI2 por debajo de los $900 \mathrm{~K}$ (ver Figura 5.12). 
Además, el parámetro $\lambda_{\mathrm{r}}$ de HFI2 es superior al de HFI1 en todo el rango de temperaturas donde coexisten las interacciones dinámicas. En el caso de $\lambda_{\mathrm{g}}$, se puede observar que entre 300 y $600 \mathrm{~K}$ la constante de recuperación de HFI1 está por encima de HFI2 mientras que por debajo de $300 \mathrm{~K}$ es mayor el parámetro $\lambda_{\mathrm{g}}$ de HFI2 (ver también Figura 5.12). En cuanto a los parámetro hiperfinos $V_{33}$ y $\eta$, los cuales caracterizan el GCE observado en el sitio de la sonda ${ }^{111} \mathrm{Cd}$, se aprecia en la Figura 5.12 que el GCE de HFI1 es constante en todo el rango de temperaturas mientras que en el caso de HFI2 se observa que presenta grandes fluctuaciones cuando la atenuación dinámica está presente.

A partir de los cálculos APW+lo se correlacionó la interacción HFI1 con el estado de carga de celda cargada mientras que para caracterizar HFI2 se realizó un estudió del GCE en función del agregado de electrones a la supercelda variando la carga con uno resolución de 0.1 electrones (ver Figura 8.1). Se pudo observar que $V_{33}$ y $\eta$ presentan grandes cambios principalmente cuando la carga varía entre el primer y segundo electrón agregado a la celda. De este modo, se concluyó que el estado final estable de HFI2 cambia con la temperatura debido a que al concluir el proceso dinámico en el entorno del Cd la totalidad de los huecos electrónicos producidos en el proceso de AE no han sido ocupados.

Por lo tanto, a partir del doble abordaje experimental y de primeros principios aplicado a este óxido puede concluirse que en el caso de HFI1 los valores más bajos de $\lambda_{\mathrm{r}}$ ajustados respecto a los de HFI2 se correlacionan con la estabilidad del estado de carga asignado a HFI1 en todo el rango de temperaturas. Mientras que los altos valores alcanzados por el parámetro $\lambda_{\mathrm{r}}$ de HFI2 y los diferentes estados estables alcanzados en función de la temperatura se corresponden con la fuerte dependencia del GCE calculada en función del agregado de carga, en particular cuando la carga varía entre 1 y 2 electrones. En el caso de $\lambda_{\mathrm{g}}$ no se le asocia relación con la dependencia funcional del GCE con el estado de carga de la supercelda.

Finalmente, a muy altas temperaturas (por encima de los $1000 \mathrm{~K}$ ) la interacción HFI2 se vuelve despreciable debido a prácticamente la totalidad de las sondas llegan dos electrones (agregados a la celda neutra) para ocupar los huecos electrónicos localizados esencialmente en el entorno del Cd y sus vecinos $\mathrm{O}_{\mathrm{NN}}$ (ver Fig. 8.14).Cabe destacar que este proceso es lo suficientemente rápido como para no alcanzar la ventana temporal de la medida PAC. Además, de lo discutido en la sección 8.5.4 se concluye 
que las grandes variaciones observadas en la interacción HFI2 en todo el rango de temperatura se correlacionan con la alta localización de los electrones agregados a la celda neutra en el entorno del $\mathrm{Cd}$ y sus $\mathrm{O}_{\mathrm{NN}}$ de modo que los cambios locales en la densidad de carga producen grandes variaciones en el GCE final estable de la interacción HFI2 para distintas temperaturas.

\section{8-6-3 Modelo semi-empírico - ab initio aplicado al óxido SnO}

En este caso se analizan las interacciones dinámicas observadas en el óxido SnO dopado con Cd. Teniendo en cuenta que en este sistema se realizaron dos ajustes diferentes de las medidas PAC, en esta sección describiremos el segundo ajuste realizado debido a que resultó más exacto que el primero puesto que se le atribuyó un conjunto de parámetros dinámicos independiente para cada interacción medida, mientras que en el primero de los ajustes se asignó el mismo grupo de parámetros dinámicos a las dos interacciones hiperfinas observadas.

A partir del análisis de las medidas experimentales, se obtuvieron dos interacciones hiperfinas constantes (HFI1 y 2) en todo el rango de temperatura. La interacción hiperfina mayoritaria HFI1 resultó ser dinámica, mientras que la interacción HFI2 presentó carácter estático en todo el rango de temperatura. Recordemos también que por encima de 700 K, HFI1 deviene en una interacción estática mientras que la mayor atenuación fue observada entres 300 y $700 \mathrm{~K}$. Finalmente, por debajo de los $300 \mathrm{~K}$ la atenuación disminuía parcialmente debido a una disminución de la vida media de los huecos $\left(\tau_{\mathrm{g}}\right)$ en el entorno del Cd.

A partir de los cálculos APW+lo se determinó que solo la aproximación LDA resultó satisfactoria para predecir las medidas PAC realizadas en el SnO:Cd. Se obtuvo una fuerte dependencia del GCE calculado en el sitio de la impureza Cd con el estado de carga de la celda. Además, a la interacción HFI1 se le asignó el resultado del cálculo correspondiente a celda neutra y a HFI2 de la celda descargada, es decir con un hueco electrónico atrapado en el entorno del Cd.

Por lo tanto, vía el doble abordaje aplicado a este sistema se asignaron dos estados de carga de la celda a las dos interacciones ajustadas en las medidas PAC. En cuanto al comportamiento dinámico de las interacciones, se pudo confirmar que el menor porcentaje de las sondas que experimentan la interacción HFI2, estática en todo el rango de temperatura, presentan en su entorno un hueco electrónico estable. En el caso de HFI1, el parámetro $\lambda_{\mathrm{r}}$ ajustado, el cual es muy importante en el rango de temperaturas 
donde la atenuación dinámica es apreciable, se correlaciona con la fuerte dependencia del GCE con el estado de carga de la supercelda. Finalmente, de lo discutido en la sección 8-5-6 se deduce que el hueco electrónico da lugar a la interacción HFI2 y que es llenado por el electrón que llega a la celda descargada, el cual se encuentra distribuido homogéneamente en los oxígenos de la supercelda, excepto en los $\mathrm{O}_{\mathrm{NN}}$ de la impureza Cd, mostrando su carácter deslocalizado. Sin embargo, es capaz de perturbar el entorno de la sonda-impureza dando lugar a la interacción HFI1 y a su carácter dinámico.

\section{8-6-4 Modelo semi-empírico - ab initio aplicado al óxido ZnO}

En este apartado se aborda el caso del óxido ZnO dopado con sondas-impurezas

${ }^{111} \mathrm{Cd}$. Las medidas PAC realizadas fueron ajustadas con una sola interacción hiperfina la cual se mantuvo constante en todo el rango de temperaturas. Sólo en las medidas a 77 y 1075 K no se observó atenuación dinámica, mientras que entre en el rango 295 - 1000 $\mathrm{K}$ un débil amortiguamiento dinámico fue observado en los espectros $\mathrm{R}(\mathrm{t})$. Cabe destacar que si bien el parámetro $\lambda_{\mathrm{r}}$ ajustado es muy apreciable (del orden del parámetro $\lambda_{\mathrm{r}}$ ajustado en el sitio $\mathrm{C}$ de las bixbitas), la atenuación resultó ser débil debido a que el parámetro $\lambda_{\mathrm{g}}$ observado resultó muy importante por lo que la vida media de los huecos en el entorno del Cd es tan pequeña que no llega a alcanzar la ventana temporal PAC. Además, los parámetros hiperfinos que caracterizan el tensor GCE ( $\mathrm{V}_{33}$ y $\left.\eta\right)$ en el sitio del Cd se mantienen constantes en todo el rango de temperaturas de medida [Muñoz 2010].

A partir de los cálculos APW+lo, a la interacción ajustada se le asignó el estado de carga de celda neutra. Además, la dependencia del GCE calculada como función del estado de carga de la supercelda resultó ser muy importante. Este hecho se correlaciona con los altos valores de la constante de relajación de Abragam y Pound, $\lambda_{\mathrm{r}}$, ajustados. Del estudio de la localización electrónica en este sistema (ver sección 8-8-5) se obtuvo que el electrón removido a la celda neutra está deslocalizado, sin embargo la creación de dicho hueco es capaz de perturbar a la impureza dando lugar a un cambio de aproximadamente $30 \%$ en el GCE calculado. 


\section{8-7 Análisis de las relajaciones estructurales}

En este apartado discutimos las relajaciones estructurales introducidas por la impureza Cd en los sistemas huéspedes abordados. Cabe destacar que en esta sección se analizan las relajaciones en las superceldas con los estados de carga correspondientes a las asignaciones experimentales realizadas a altas temperaturas, es decir donde las interacciones hiperfinas son estáticas.

En primera instancia analicemos las relajaciones introducidas por el $\mathrm{Cd}$ en los óxidos con estructura bixbita. En la Tabla 8.7 se detallan las distancias $\mathrm{Cd}-\mathrm{O}_{\mathrm{NN}}$ relajadas y no relajadas correspondientes a los óxidos abordados en la presente Tesis (los sesquióxidos $\mathrm{Sc}_{2} \mathrm{O}_{3}, \mathrm{Y}_{2} \mathrm{O}_{3}$ e $\mathrm{In}_{2} \mathrm{O}_{3}$; y los óxidos $\mathrm{SnO}_{2}$, $\mathrm{SnO}$ y $\mathrm{ZnO}$ ).

A partir de las relajaciones estructurales tabuladas se aprecia que las distancias $d_{C d-}$ ONN tienden a la distancia Cd-O de su propio óxido (2.35 $\AA$ en CdO). En el caso particular de Cd localizado en sitio $C$, se puede observar que los oxígenos $\mathrm{O}_{N N i}(i=1,2$, 3) mantienen su relación respecto del caso no relajado. Además se aprecia que cuanto más pequeño es el radio iónico del catión nativo (ver Tabla 8.8), la proporción de la dilatación es mayor. En el caso de Cd en sitio C se observan las siguientes proporciones en las dilataciones: en $\mathrm{Sc}_{2} \mathrm{O}_{3}$ las distancias $\mathrm{Cd}-\mathrm{O}_{N N 1}$ se dilatan $5 \%$ respecto del caso no relajado, para $d_{C d-O N N 2}$ la dilatación es de $9 \%$ y para $d_{C d-O N N 3}$ es de $7 \%$; en $\operatorname{In}_{2} \mathrm{O}_{3} d_{C d-O N N 1}$ relaja el $4 \%$, mientras que $d_{C d-O N N 2}$ y $d_{C d-O N N 3}$ dilata el $5 \%$; finalmente en el caso de $\mathrm{Y}_{2} \mathrm{O}_{3} d_{C d-O N N 1}$ relaja el 1\%, $d_{C d-O N N 2}$ dilata el $4 \%$ y dCd-ONN3 el 5\%. En el caso de Cd en sitio D se tiene: en $\mathrm{Sc}_{2} \mathrm{O}_{3} d_{C d-O N N}$ relaja un 7\%, en $\mathrm{In}_{2} \mathrm{O}_{3}$ un $4 \%$ y en $\mathrm{Y}_{2} \mathrm{O}_{3}$ un $3 \%$.

En el caso de $\mathrm{SnO}$ se puede observar que la dilatación producida por el Cd es tal que la distancia relajada $d_{C d-O}$ y es muy cercana a la distancia $\mathrm{Cd}-\mathrm{O}$ de su óxido $\left(\mathrm{CdO}, d_{C d-}\right.$ $o^{=}=2.35 \AA$ ). Si bien los radios iónicos del $\mathrm{Sn}^{2+} \mathrm{y} \mathrm{Cd}^{2+}$ son similares (ver Tabla 8.8), el Cd introduce relajaciones buscando una ligadura similar a la del CdO y esta estructura “abierta” se lo permite. Además, desde esta tabla de radios iónicos se aprecia que los radios más pequeños corresponden al $\mathrm{Sn}^{4+} \mathrm{y}$ al $\mathrm{Zn}^{2+}$, y contrastando con los resultados mostrados en la tabla 8.7 se aprecia que las estructuras del $\mathrm{SnO}_{2}$ y el $\mathrm{ZnO}$ son las que presentan menores dilataciones. por lo tanto, se concluye que las estructuras que presentan menores distancias catión-oxígeno (menores radios iónicos del catión nativo) ofrecen más oposición a la deformación estructural producida por la inclusión de la impureza Cd en la matriz huésped. 


\begin{tabular}{|c|c|c|c|}
\hline \multicolumn{4}{|c|}{ Cd en Sitio C } \\
\hline Óxido & $\mathbf{O}_{\mathrm{NN}}$ & $d_{N N}$ relajada & $d_{N N}$ no relajada \\
\hline \multirow{3}{*}{$\mathrm{Sc}_{2} \mathrm{O}_{3}$} & $\mathrm{O}_{\mathrm{NN} 1}$ & 2.19 & 2.08 \\
\hline & $\mathrm{O}_{\mathrm{NN2}}$ & 2.31 & 2.12 \\
\hline & $\mathrm{O}_{\mathrm{NN3}}$ & 2.32 & 2.16 \\
\hline \multirow{3}{*}{$\mathrm{In}_{2} \mathrm{O}_{3}$} & $\mathrm{O}_{\mathrm{NN} 1}$ & 2.20 & 2.12 \\
\hline & $\mathrm{O}_{\mathrm{NN} 2}$ & 2.30 & 2.19 \\
\hline & $\mathrm{O}_{\mathrm{NN3}}$ & 2.32 & 2.21 \\
\hline \multirow{3}{*}{$\mathrm{Y}_{2} \mathrm{O}_{3}$} & $\mathrm{O}_{\mathrm{NN} 1}$ & 2.27 & 2.24 \\
\hline & $\mathrm{O}_{\mathrm{NN} 2}$ & 2.36 & 2.27 \\
\hline & $\mathrm{O}_{\mathrm{NN} 3}$ & 2.45 & 2.33 \\
\hline \multicolumn{4}{|c|}{ Cd en Sitio D } \\
\hline $\mathrm{Sc}_{2} \mathrm{O}_{3}$ & $\mathrm{O}_{\mathrm{NN}}$ & 2.28 & 2.12 \\
\hline $\mathrm{In}_{2} \mathrm{O}_{3}$ & $\mathrm{O}_{\mathrm{NN}}$ & 2.28 & 2.19 \\
\hline $\mathrm{Y}_{2} \mathrm{O}_{3}$ & $\mathrm{O}_{\mathrm{NN}}$ & 2.36 & 2.28 \\
\hline Óxido & $\mathbf{O}_{\mathrm{NN}}$ & $d_{N N}$ relajada & $d_{N N}$ no relajada \\
\hline \multirow{2}{*}{$\mathrm{SnO}_{2}$} & $\mathrm{O}_{\mathrm{NN} 1}$ & 2.15 & 2.05 \\
\hline & $\mathrm{O}_{\mathrm{NN} 2}$ & 2.20 & 2.06 \\
\hline \multirow{2}{*}{$\mathrm{ZnO}$} & $\mathrm{O}_{\mathrm{NN} 1}$ & 2.16 & 1.98 \\
\hline & $\mathrm{O}_{\mathrm{NN} 2}$ & 2.19 & 1.99 \\
\hline $\mathrm{SnO}$ & $\mathrm{O}_{\mathrm{NN}}$ & 2.37 & 2.22 \\
\hline
\end{tabular}

Tabla 8.7: Comparación entre las distancias $\mathrm{Cd}_{-} \mathrm{O}_{\mathrm{NN}}$ para los casos de celda relajada y no relajada (cálculos APW+lo) en los óxidos con estructura bixbita y en los óxidos $\mathrm{SnO}_{2}$, $\mathrm{SnO}$ y $\mathrm{ZnO}$ dopados con $\mathrm{Cd}$.

\begin{tabular}{cc}
\hline \hline Catión & $\boldsymbol{r}_{\text {ión }}(\AA)^{(\mathbf{a})}$ \\
\hline $\mathrm{Cd}^{2+}$ & 0.95 \\
\hline $\mathrm{Y}^{3+}$ & 0.9 \\
\hline $\mathrm{In}^{3+}$ & 0.8 \\
\hline $\mathrm{Sc}^{3+}$ & 0.745 \\
\hline $\mathrm{Sn}^{2+}$ & 0.94 \\
\hline $\mathrm{Sn}^{4+}$ & 0.69 \\
\hline $\mathrm{Zn}^{2+}$ & 0.6 \\
\hline \hline
\end{tabular}

Tabla 8.8: radios iónicos de los cationes nativos y del ión $C d^{2+}$.
En la Figura 8.25 se describen las orientaciones de las dilataciones introducidas por el Cd en su coordinación espacial con sus primeros vecinos oxígeno para cada óxido.

Para el caso de Cd situado en los sitios catiónicos (Fig. 8.25 (a) y (b)) de los óxidos con estructura bixbita se aprecia que las direcciones de relajación distan bastante de las direcciones de las ligaduras $\mathrm{Cd}-\mathrm{O}_{\mathrm{NN}}$. Esto se debe a la oposición que ofrecen los cationes nativos (primeros vecinos de los átomos oxígeno) al desplazamiento de los primeros vecinos $\mathrm{O}_{\mathrm{NN}}$ del Cd.

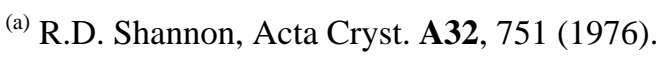


(a)

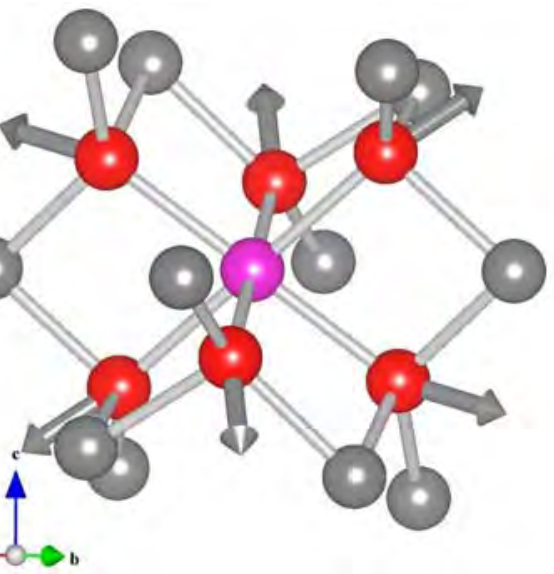

(c)

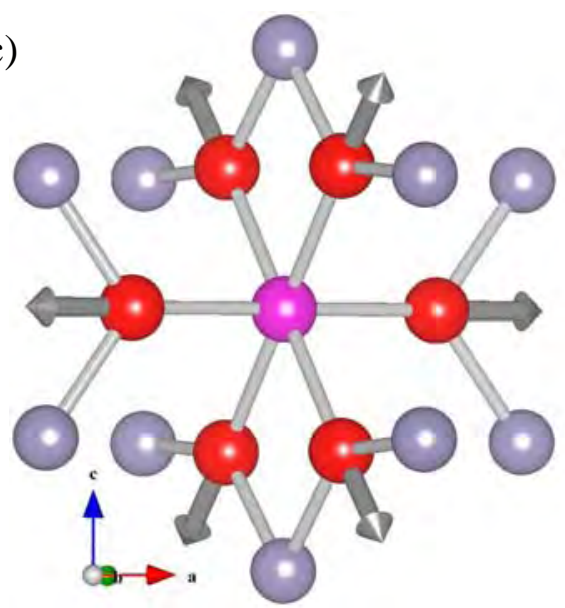

(e)

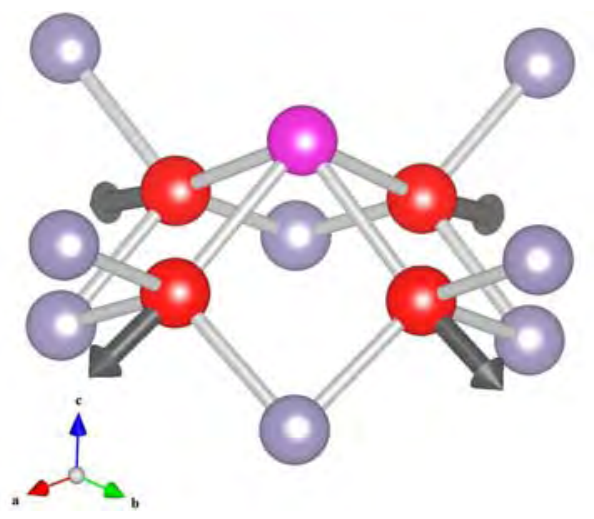

(b)

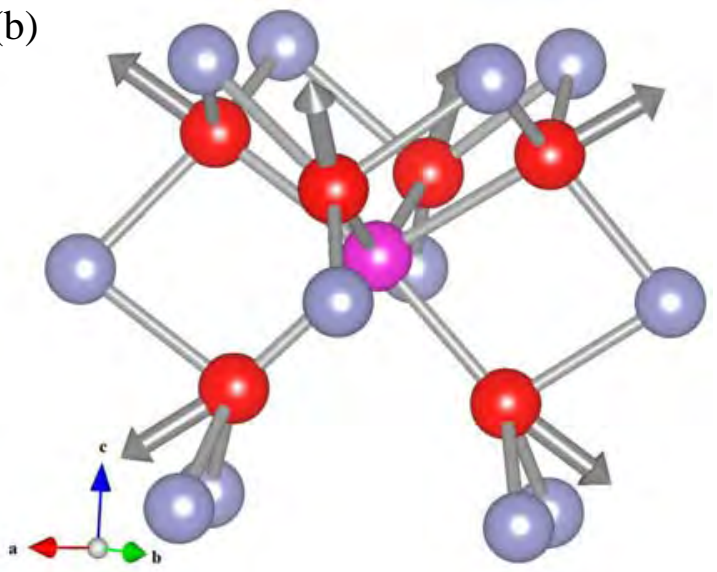

(d)

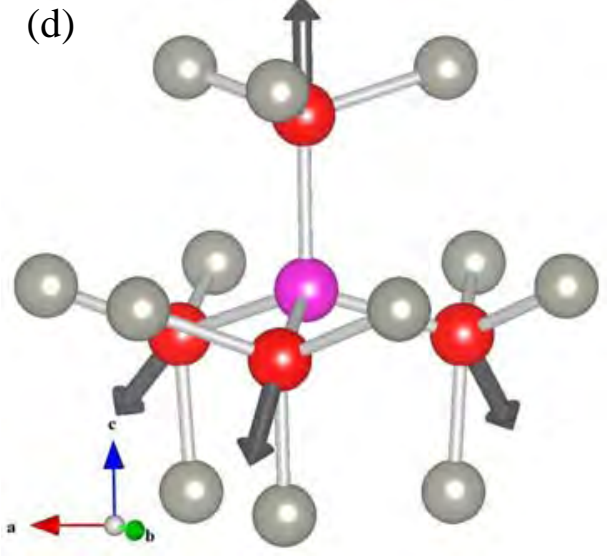

Figura 8.25: Representación de las relajaciones de los $\mathrm{O}_{\mathrm{NN}}$ en la red huésped (vectores de color gris) y coordinación NN de la impureza Cd (esfera de color magenta) con sus primeros vecinos oxígeno (esferas rojas) y de estos con sus cationes NN (esferas grises). (a) Sitio $\mathrm{D}$ de la bixbitas; (b) sitio C de la bixbita; (c) $\mathrm{SnO}_{2}$; (d) $\mathrm{ZnO} ; \mathrm{y}$ (e) $\mathrm{SnO}$.

En el caso de $\mathrm{SnO}_{2}:{ }^{111} \mathrm{Cd}$ (Fig. 8.25 (c)), se puede observar que las relajaciones de los $\mathrm{O}_{\mathrm{NN} 1}$, los cuales se sitúan en el eje del octaedro, apuntan en la dirección de dicho eje; mientras que los átomos oxígeno $\mathrm{O}_{\mathrm{NN} 2}$, situados en los vértices del plano del octaedro, se desplazan en las direcciones de las ligaduras $\mathrm{Cd}_{-} \mathrm{O}_{\mathrm{NN} 2}$. Esto está en acuerdo con la oposición simétrica ofrecida por los átomos Sn (primeros vecinos de los átomos oxígeno) a la distorsión estructural debido a la configuración simétrica de los cationes Sn con los oxígenos $\mathrm{O}_{\mathrm{NN} 1}$ y $\mathrm{O}_{\mathrm{NN} 2}$. 
En el $\mathrm{ZnO}:{ }^{111} \mathrm{Cd}$ (Fig. 8.25 (d)) se observa que las direcciones de las relajaciones de los 3 átomos oxígeno $\mathrm{O}_{\mathrm{NN} 1}$ (que están por debajo del $\mathrm{Cd}$ ) presentan una inclinación hacia abajo respecto de la dirección de las ligaduras $\mathrm{Cd}-\mathrm{O}_{\mathrm{NN} 1}$, debido a la repulsión ofrecida por los vecinos $\mathrm{Zn}$ de los $\mathrm{O}_{\mathrm{NN} 1}$ tal como se aprecia en la figura. Además se observa que la dirección de la ligadura $\mathrm{O}_{\mathrm{NN} 1}-\mathrm{Zn}$ del átomo $\mathrm{Zn}$ inferior a cada oxígeno $\mathrm{O}_{\mathrm{NN} 1}$ está levemente inclinado respecto de la dirección vertical como consecuencia de la distorsión estructural producida por la inclusión de la impureza Cd en su entorno cercano. En el caso del vecino superior $\mathrm{O}_{\mathrm{NN} 2}$, de acuerdo a la simetría del sitio presenta un desplazamiento en la dirección del eje $c$.

En el caso de $\mathrm{SnO}:{ }^{111} \mathrm{Cd}$ (Fig. 8.25 (e)) se observa que la dirección de los desplazamientos presenta un débil corrimiento de la ligadura $\mathrm{Cd}-\mathrm{O}_{\mathrm{NN}}$ lo cual se correlaciona con la repulsión al desplazamiento de los dos Sn vecinos inferiores de cada oxígeno $\mathrm{O}_{\mathrm{NN}}$, la cual es predominante por sobre la oposición del Sn vecino superior de cada $\mathrm{O}_{\mathrm{NN}}$.

\section{Referencias}

[Lupascu 1994] D. Lupascu, A. Bartos, K. P. Lieb and M. Uhrmacher, Z. Phys. B 93, 441 (1994).

[Rita 2005] E. Rita, J. G. Correia, U. Wahl, E. Alves, A. M. L. Lopes, J. C. Soares and The ISOLDE Collaboration, Hyperfine Interact. 158, 395 (2005).

[Muñoz 2011] E.L. Muñoz, D. Richard, A.W. Carbonari, L.A. Errico and M. Rentería, Hyperfine Interact. 197, 199 (2011).

[Bäverstam 1972] U. Bäverstam, R. Othaz, N. De Sousa and B. Ringström, Nucl. Phys. A186, 500 (1972).

[Muñoz 2011b] E.L. Muñoz, D. Richard, P.D. Eversheim and M. Rentería, Hyperfine Interact. 197, 187 (2011).

[Muñoz 2010] E.L. Muñoz, M.E. Mercurio, M.R. Cordeiro, L.F.D. Pereira, A.W. Carbonari, and M. Rentería, Physica B (2010) (enviado para su publicación). 


\section{Capítulo Nº}

\section{Conclusiones}

En el presente trabajo de Tesis se ha realizado un estudio experimental y de primeros principios de interacciones hiperfinas dinámicas observadas por sondasimpurezas $\left({ }^{111} \operatorname{In}(\mathrm{EC}) \rightarrow\right)^{111} \mathrm{Cd}$ localizadas sustitucionalmente en sitios catiónicos de óxidos semiconductores con variadas estructuras cristalinas y por ende diferentes geometrías de coordinación de la impureza con sus primeros vecinos oxígeno. El objetivo fundamental del trabajo consistió en elaborar un modelo semi-empírico - $a b$ initio combinando resultados de experimentos PAC que presentan interacciones hiperfinas dinámicas y cálculos FP-APW+lo del tensor GCE (calculado en el sitio de la impureza) como función del estado de carga de la supercelda, capaz de describir un escenario compatible con la aparición de las interacciones dinámicas experimentadas por la sonda $\left({ }^{111} \mathrm{In} \rightarrow\right)^{111} \mathrm{Cd}$ y atribuidas a los procesos de relajación electrónica (aftereffects) posteriores al decaimiento por EC del isótopo padre ${ }^{111} \mathrm{In}$. Paralelamente, otro objetivo primordial de la Tesis fue caracterizar los GCEs observados por el núcleosonda en cada uno de los óxidos semiconductores dopados seleccionados a partir del excelente acuerdo entre los resultados PAC y las predicciones obtenidas en los cálculos $a b$ initio. Finalmente, a partir de la buena comparación entre teoría y experimento se realizó una exhaustiva descripción de las propiedades estructurales y electrónicas obtenidas a partir del estudio FP-APW+lo de estructura electrónica de los sistemas dopados con la impureza Cd.

Desde el punto de vista experimental se ha caracterizado el tensor GCE observado en sitios de impureza $\left({ }^{111} \mathrm{In}(\mathrm{EC}) \rightarrow{ }^{111} \mathrm{Cd}\right.$ en los sesquióxidos $\mathrm{Sc}_{2} \mathrm{O}_{3}, \mathrm{In}_{2} \mathrm{O}_{3}$ e $\mathrm{Y}_{2} \mathrm{O}_{3}$; en los óxidos de Estaño, $\mathrm{SnO}_{2}$ y $\mathrm{SnO}$; y en $\mathrm{ZnO}$. En particular, se midió la dependencia del GCE en función de la temperatura en un amplio rango y en forma detallada. En los casos de medidas previas existentes en la literatura $\left(\mathrm{In}_{2} \mathrm{O}_{3}, \mathrm{Sc}_{2} \mathrm{O}_{3}, \mathrm{Y}_{2} \mathrm{O}_{3}, \mathrm{SnO}_{2} \mathrm{y} \mathrm{ZnO}\right)$, nuestros resultados experimentales están en perfecto acuerdo con los valores reportados. En el caso particular del óxido $\mathrm{Sc}_{2} \mathrm{O}_{3}$, en la literatura solo se había reportado el GCE a $650 \mathrm{~K}$, mientras que del $\mathrm{Y}_{2} \mathrm{O}_{3}$ se habían exhibido los espectros $\mathrm{PAC}$ medidos en el rango 295 - $950 \mathrm{~K}$ y los parámetros hiperfinos a alta temperatura. En todos los óxidos 
estudiados en esta Tesis, excepto en el $\mathrm{ZnO}$, una gran atenuación dinámica fue observada a temperaturas intermedias. Esta atenuación resultó ser muy apreciable hasta temperaturas muy bajas salvo en el caso del $\operatorname{In}_{2} \mathrm{O}_{3}$ donde la señal se recupera apreciablemente a $100 \mathrm{~K}$. En el caso del ZnO una débil atenuación fue observada en todo el rango de temperatura excepto en los extremos del rango, a 77 y $1075 \mathrm{~K}$ donde la interacción permanece estática. En los óxidos con estructura bixbita, se han caracterizado dos interacciones, una de simetría axial $(\eta=0)$ y la otra con alto valor de $\eta$ por lo que se las atribuyó a sondas ${ }^{111} \mathrm{Cd}$ localizadas en los sitios D y C de la estructura, respectivamente. En el óxido $\mathrm{SnO}_{2}$ fueron caracterizadas dos interacciones, una de ellas coincidente con las medidas reportadas en la literatura. En el monóxido SnO también se caracterizaron dos interacciones hiperfinas las cuales coexisten en todo el rango de temperatura. Por último, en $\mathrm{ZnO}$ se caracterizó una sola interacción axialmente simétrica acorde a la simetría puntual del sitio catiónico.

A partir de los cálculos FP-APW+lo realizados en los seis óxidos mencionados en el párrafo anterior se pudieron confirmar las asignaciones realizadas en el análisis de los experimentos PAC de los óxidos con estructura bixbita (asignaciones basadas en la simetría axial del sitio $\mathrm{D}$, en la alta asimetría que caracteriza al sitio $\mathrm{C}$ y en la distribución homogénea de las sondas en la estructura cristalina); en $\mathrm{SnO}_{2}$ se pudieron interpretar las dos interacciones observadas a partir de un estudio exhaustivo del GCE en función del estado de carga de la celda (con la particularidad de que la variación de la carga agregada a la celda fue de $0.1 e^{-}$) y del análisis de las interacciones dinámicas realizado con el modelo de Bäverstam y Othaz en el ajuste de los espectros PAC; en el caso del SnO, también a partir del doble abordaje experimental-ab initio del GCE se pudieron interpretar las dos interacciones observadas; y finalmente, en $\mathrm{ZnO}$, la débil atenuación dinámica encontrada pudo ser interpretada a la luz de los cálculos ab initio y de los parámetros dinámicos del ajuste.

A partir del estudio $a b$ initio realizado a lo largo del trabajo se demostró que es necesario considerar las relajaciones estructurales que introduce la impureza $\mathrm{Cd}$ en la red huésped para poder reproducir correctamente el GCE determinado por las medidas experimentales.

Del análisis de la densidad de carga electrónica que contribuye al nivel de impureza, se demostró que en aquellos óxidos donde el ión $\mathrm{Cd}^{2+}$ no es isovalente con el catión nativo (casos de impureza simple y doble aceptora) tales estados electrónicos son altamente localizados en el entorno del $\mathrm{Cd}$ y sus $\mathrm{O}_{\mathrm{NN}}$, mientras que en aquellos sistemas 
donde el $\mathrm{Cd}^{2+}$ es isovalente, estos estados electrónicos son esencialmente deslocalizados.

A partir del estudio de las densidades de estados (DOS) totales se pudieron correlacionar las contribuciones a las bandas de valencia y de conducción de los sistemas abordados en función del carácter de los enlaces catión-oxígenos (iónico o covalente) y de la forma de las estructuras y su empaquetamiento como así también de la coordinación (NN) del catión.

Del estudio de las densidades de estado parciales (PDOS) en la región de los niveles de impureza se concluyó que las contribuciones relativas de las simetrías $s, p$ y $d$ que aportan a los estados de impureza del Cd son fuertemente dependientes del carácter de la impureza (isovalente, donora o aceptora) al reemplazar un ión nativo del óxido en cuestión. Así mismo, se comprobó que la ubicación de los niveles de impureza en la DOS del sistema depende del carácter de la impureza en la red huésped.

Del análisis de las PDOS de las simetrías $d$ del Cd en la región del nivel de impureza, se pudieron correlacionar la forma relativa de las contribuciones de las distintas simetrías con la dependencia del GCE con el estado de carga de la celda.

A partir del estudio de las contribuciones $s-d, p$ y $d$ al GCE calculado en el sitio de la impureza se concluye que, en general, las contribuciones $p$ (electrones $5 p$ del Cd) son más importantes en magnitud que las contribuciones $d$ (electrones $4 d$ del $\mathrm{Cd}$ ) debido a que los estados $p$ están más cerca del núcleo del Cd. Recordemos que el número de nodos de la función de onda radial viene dado por $n-l-1$, luego la función de onda que presente más nodos concentrará más carga cerca del núcleo. Adicionalmente, se concluye que la dependencia funcional de las contribuciones $s-d, p$ y $d$ al GCE con el estado de carga de la celda es fuertemente dependiente de la simetría del sitio catiónico donde se aloja la impureza.

En todos los sistemas abordados se observó que la introducción del Cd en la red huésped introduce relajaciones estructurales en su entorno cercano (primeros vecinos oxígeno) que tienden a reproducir la distancia $\mathrm{Cd}-\mathrm{O}_{\mathrm{NN}}$ de su propio óxido, el CdO. En los óxidos con estructura bixbita y el SnO se aprecia que la relajación estructural es más importante que en el caso de los óxidos $\mathrm{SnO}_{2}$ y ZnO. Este hecho fue atribuido a la diferencia entre las distancias catión- $\mathrm{O}_{\mathrm{NN}}$ existente entre los sesquióxidos y el $\mathrm{SnO}$ respecto a los óxidos $\mathrm{SnO}_{2}$ y $\mathrm{ZnO}$. 
Se comprobó que las distancias $\mathrm{Cd}-\mathrm{O}_{\mathrm{NN}}$ se ven aumentadas al agregar electrones a la celda, en acuerdo con el incremento de la repulsión Coulombiana, y que dicha dilatación es más apreciable en los casos en que el electrón es más localizado.

Se comprobó que mediante el análisis combinado de las DOS y de la densidad electrónica $\rho(\boldsymbol{r})$ se puede determinar fehacientemente la localización espacial de la carga agregada o sustraída en la supercelda. Dicha carga, en general, contribuye al llenado o vaciado del nivel de impureza, pero presenta distintas localizaciones espaciales dependiendo del carácter de la impureza en la red huésped.

A partir de la comparación entre los resultados experimentales y las predicciones realizadas con el modelo PCM se concluye una vez más que este modelo es obsoleto para describir interacciones hiperfinas observadas por impurezas localizadas sustitucionalmente en óxidos semiconductores debido a que no tiene en cuenta el carácter de impureza de la sonda en la red del huésped ya que solo considera la interacción del átomo-sonda con su entorno a través del factor de anti-apantallamiento de Sternheimer.

En el caso de las óxidos con estructura bixbita, mediante el doble abordaje experimental y de primeros principios se pudo establecer el estado de carga de la sondaimpureza ${ }^{111} \mathrm{Cd}$ a altas temperaturas. En el caso particular del $\mathrm{In}_{2} \mathrm{O}_{3}$, los parámetros hiperfinos de las interacciones observadas se mantuvieron constantes en todo el rango de temperatura por lo que el estado de carga final estable de la impureza (estado de carga doble aceptor ionizado completamente), luego del proceso de relajación electrónica, no presentó variaciones con la temperatura. En el caso del $\mathrm{Sc}_{2} \mathrm{O}_{3}$, la variación de los parámetros hiperfinos $V_{33}$ y $\eta$ con la temperatura pudo ser correlacionada cualitativamente en función del cambio de la carga en el entorno de la impureza, pero para una correcta determinación del estado de carga de la impureza en todo el rango de temperaturas se concluye que es necesario en el futuro realizar cálculos del GCE variando la carga agregada a la celda con una mayor resolución. Y en el caso del $\mathrm{Y}_{2} \mathrm{O}_{3}$, la variación de los parámetros hiperfinos con la temperatura permitió determinar sólo el estado de carga de la impureza a altas temperaturas por lo que se concluye que para una correcta determinación del estado de carga de la impureza en todo el rango de temperatura también es necesario calcular la dependencia del GCE con el estado de carga considerando una mayor resolución en la carga agregada a la supercelda. Cabe destacar que el estado final estable de la sonda en los sesquióxidos 
$\mathrm{Sc}_{2} \mathrm{O}_{3}$ e $\mathrm{Y}_{2} \mathrm{O}_{3}$ es diferente para cada temperatura, lo que se traduce en la dependencia del GCE ajustado en función de la temperatura.

A partir de la aplicación del factor de perturbación dinámico en los ajustes de las medidas PAC en los óxidos con estructura bixbita se concluye que la atenuación dinámica observada en los espectros es producida esencialmente por las sondasimpurezas ${ }^{111} \mathrm{Cd}$ localizadas en el sitio C. A partir del doble abordaje experimental-ab initio aplicado a las bixbitas se concluye que la atenuación dinámica atribuida al sitio C es producto de la gran asimetría del mismo, mientras que la débil contribución (a la relajación dinámica de los espectros) observada en el sitio D se correlaciona con la alta simetría que presenta dicho sitio. En cuanto a la vida media de los huecos electrónicos en los estados de impureza se determinó que es independiente del sitio (C o D) donde se localiza la sonda en la estructura de estos semiconductores. Por último, se concluye que por debajo de los $700 \mathrm{~K}(600 \mathrm{~K})$ en los óxidos de $\mathrm{Y}_{2} \mathrm{O}_{3}$ y $\mathrm{Sc}_{2} \mathrm{O}_{3}\left(\mathrm{In}_{2} \mathrm{O}_{3}\right)$ los huecos electrónicos son ocupados durante la ventana temporal PAC, mientras que por encima de esta temperatura la ocupación de los huecos ocurre antes de la ventana PAC. Cabe destacar que en el caso particular del $\mathrm{In}_{2} \mathrm{O}_{3}$ la vida media de los huecos electrónicos por debajo de $100 \mathrm{~K}$ disminuye apreciablemente lo que se evidencia en una recuperación parcial de la señal de los espectros PAC.

En el caso del óxido $\mathrm{SnO}_{2}$, como se mencionó anteriormente, dos interacciones hiperfinas fueron ajustadas en todo el rango de temperatura. Las dos interacciones resultaron dinámicas a temperaturas bajas e intermedias, HFI1 por debajo de $600 \mathrm{~K}$ y HFI2 por debajo de $900 \mathrm{~K}$. A partir de la buena comparación entre experimento y teoría se dedujo que la interacción que resultó ser mayoritaria a altas y bajas temperaturas (HFI1) corresponde al estado de carga de la impureza cargado. Por debajo de $600 \mathrm{~K}$, los huecos electrónicos son ocupados en un tiempo suficientemente largo como para alcanzar la ventana temporal PAC, mientras que por encima de $600 \mathrm{~K}$ este proceso es más rápido de modo de no alcanzar la ventana PAC. En cuanto a la segunda interacción (HFI2), la cual se hace mayoritaria en una pequeña ventana de temperaturas intermedias, presentó una gran variación en sus parámetros hiperfinos $V_{33}$ y $\eta$. Por debajo de $900 \mathrm{~K}$, los huecos electrónicos son compensados parcialmente en un tiempo mayor a la ventana temporal PAC, y por encima de $900 \mathrm{~K}$, los huecos tienden a ser ocupados totalmente y en un intervalo de tiempo menor que la ventana PAC. La interacción HFI2 fue totalmente interpretada a partir de un estudio APW+lo del GCE 
variando la carga con una resolución de $0.1 e^{-}$, con lo que se concluyó que los cambios observados en $V_{33}$ y $\eta$ de HFI2 se debe a que la carga agregada a la celda (la cual debe ionizar los huecos electrónicos para culminar el proceso dinámico observado) cambia en función de la temperatura dando lugar a cambios en el GCE final estable ajustado en cada temperatura de medida. La dependencia de los parámetros dinámicos ajustados en función de la temperatura se pudo correlacionar satisfactoriamente con la dependencia del GCE con el estado de carga de la impureza para cada interacción. En el caso de la dependencia funcional de la vida media de los huecos con la temperatura se observó que no depende del estado de carga de la impureza.

En el caso del SnO, de las dos interacciones hiperfinas ajustadas (HFI1 y HFI2) sólo la interacción mayoritaria presentó un carácter dinámico. A partir del doble abordaje realizado, a la interacción mayoritaria HFI1 se le asignó el estado de carga neutro, tanto a altas temperaturas donde la interacción es estática como al estado final estable en el resto del rango de temperatura (donde la interacción es dinámica), mientras que a HFI2 se le asignó el estado descargado (1 e- menos). De la aplicación del factor de perturbación dinámico al análisis de los espectros PAC se concluye que la interacción HFI2 corresponde a sondas ${ }^{111} \mathrm{Cd}$ con un hueco electrónico atrapado y parcialmente deslocalizado, cuyo estado no cambia durante la ventana temporal PAC. En cuanto a la interacción HFI1 se concluye que corresponde a sondas ${ }^{111} \mathrm{Cd}$ que alcanzan el estado neutro antes de la ventana temporal PAC a altas temperaturas y durante la misma a temperaturas intermedias.

En el caso del ZnO, a partir del doble abordaje ya descripto se caracterizó la interacción hiperfina observada (que presenta una sutil atenuación dinámica) y se le asignó unívocamente el estado de carga neutro para el estado final estable en todo el rango de temperatura (recordemos que la impureza Cd es isovalente en este caso). Cabe destacar que el GCE final estable ajustado se mantuvo constante en todo el rango de temperatura. La dependencia de los parámetros dinámicos $\left(\lambda_{\mathrm{g}} \mathrm{y} \lambda_{\mathrm{r}}\right)$ en función de la temperatura fue interpretada correctamente a la luz del cálculo de la dependencia del GCE con el estado de carga de la celda. Si bien se determinó una importante dependencia del GCE con el estado de carga, se concluye que no se observa una fuerte atenuación dinámica en los espectros $\mathrm{R}(\mathrm{t})$ debido a que el hueco electrónico es ocupado antes de (o en tiempos muy cortos respecto a) la ventana temporal de la medida PAC. 
Finalmente, el abordaje experimental- $a b$ initio aplicado en la presente Tesis, basado en el modelo de Bäverstam y Othaz para el análisis de los espectros PAC y en cálculos FP-APW+lo del tensor GCE (en el sitio de la impureza) en función del estado de carga de la supercelda, nos permitió formular un escenario que da cuenta del proceso de after-effects (debido al decaimiento por EC del isótopo padre ${ }^{111} \operatorname{In}\left((\mathrm{EC}) \rightarrow{ }^{111} \mathrm{Cd}\right)$ ) que da lugar a las interacciones hiperfinas dinámicas observadas por el núcleo ${ }^{111} \mathrm{Cd}$. En este escenario se pudo correlacionar la constante de relajación de Abragam y Pound, $\lambda_{\mathrm{r}}$, y su dependencia con la temperatura en cada sistema con la dependencia del GCE con el estado de carga de la impureza. En cuanto a la constante de recuperación, $\lambda_{\mathrm{g}}$, pudo ser interpretada como la inversa de la vida media de los huecos electrónicos $\left(\tau_{\mathrm{g}}=\lambda_{\mathrm{g}}{ }^{-1}\right)$ responsables de la interacciones dinámicas en las medida PAC. Esta interpretación nos permitió cuantificar los tiempos característicos empleados por la densidad de carga electrónica en el entorno de la impureza ${ }^{111} \mathrm{Cd}$ para alcanzar el estado final estable. 


\section{Apéndice I}

\section{I-1 Formalismo de la Matriz Densidad}

Teniendo en cuenta el carácter estadístico del proceso de decaimiento nuclear de un átomo-sonda radiactivo dopando un determinado material y la posible interacción hiperfina entre el núcleo y el campo extranuclear, trabajaremos en el formalismo de la matriz densidad. La idea de este apartado es llegar a una expresión sencilla para el factor de perturbación en la cual pueda factorizarse la parte que dependa sólo del campo extranuclear.

Supongamos que un determinado número de núcleos que están decayendo del estado inicial $\mathrm{I}_{\mathrm{i}}$ (con espín $\mathrm{I}_{\mathrm{i}}$ ) al estado intermedio I (con espín I) están descriptos por la matriz densidad $\rho_{\mathrm{i}}$. El proceso se lleva a cabo luego de emitir cada núcleo radiación $\gamma_{1}$ en la dirección $\vec{k}_{1}$. El estado intermedio I está descripto por la matriz $\rho\left(\vec{k}_{1}\right)$. La transición del estado I al estado final $\mathrm{I}_{\mathrm{f}}$ se analiza de la misma forma, entonces la matriz en este estado será $\rho_{\mathrm{f}}\left(\vec{k}_{1}, \vec{k}_{2}\right)$.

En el estado inicial $\mathrm{I}_{\mathrm{i}}$, se supone que los subestados $\left|I_{i} m_{i}\right\rangle$ están igualmente poblados debido a que solo consideramos que existe una interacción hiperfina luego de haberse emitido $\gamma_{1}$. Si $\mathrm{H}_{1}$ es el hamiltoniano de interacción entre el campo cristalino y el núcleo correspondiente a la transición $\mathrm{I}_{\mathrm{i}} \rightarrow \mathrm{I}$, entonces, los elementos de matriz de $\rho_{a}\left(\vec{k}_{1}\right)$ pueden expresarse como [Rentería 1987]:

$$
\left.\left\langle m\left|\rho_{a}\left(\vec{k}_{1}\right)\right| m^{\prime}\right\rangle=S_{1} \sum_{m_{i}}\left\langle m\left|H_{1}\right| m_{i}\right\rangle m_{i}\left|H_{1}^{+}\right| m^{\prime}\right\rangle,
$$

donde $S_{1}$ simboliza la sumatoria sobre las propiedades que no se miden en el experimento (espín, polarización de la radiación, etc).

Debido a que existe una interacción entre el GCE y el momento cuadrupolar del núcleo en el estado intermedio, la matriz $\rho_{a}\left(\vec{k}_{1}\right)$ al final de la primera transición $\mathrm{I}_{\mathrm{i}} \rightarrow \mathrm{I}$ no es igual a la matriz $\rho_{b}\left(\vec{k}_{1}\right)$ que describe el estado inicial de la segunda transición $\mathrm{I} \rightarrow$ If. El campo extranuclear causa transiciones entre los subestados $m$ del estado I, este proceso se puede esquematizar como sigue:

$$
\rho_{a}\left(\vec{k}_{1}\right) \underset{\substack{\text { interacción } \\ \text { hiperfina }}}{\rightarrow} \rho_{b}\left(\vec{k}_{1}\right)
$$


Esta interacción transcurre durante el intervalo de tiempo que el núcleo se encuentra en el estado intermedio.

Este cambio puede atribuirse a una transformación unitaria $\Lambda(\mathrm{t})$ que describa la evolución temporal de los subestados $|m\rangle$ en el estado I. Si $\mathrm{H}_{\mathrm{Q}}$ es el hamiltoniano de la perturbación extranuclear, entonces, $\Lambda(\mathrm{t})$ cumple:

$$
\frac{\partial \hat{\Lambda}(t)}{\partial t}=\frac{-i}{\hbar} \hat{H}_{Q} \hat{\Lambda}(t)
$$

De modo que la solución es:

$$
\hat{\Lambda}(t)=e^{\frac{-i}{\hbar} \int_{0}^{t} \hat{H}_{Q}\left(t^{\prime}\right) d t^{\prime}} .
$$

Según se han definido $\rho_{a}\left(\vec{k}_{1}\right)$ y $\rho_{b}\left(\vec{k}_{1}\right)$, suponiendo que el núcleo se encuentra un tiempo t en el estado I, se puede escribir $\rho_{a}\left(\vec{k}_{1}\right)=\rho\left(\vec{k}_{1}\right)$ y $\rho_{b}\left(\vec{k}_{1}\right)=\rho\left(\vec{k}_{1}, t\right)$. De este modo, la matriz densidad que describe el sistema en el estado inicial del segundo proceso se puede expresar en función del operador evolución temporal $\Lambda(\mathrm{t})$ como [Frauenfelder, 1965]:

$$
\begin{gathered}
\hat{\rho}\left(\vec{k}_{1}, t\right)=\hat{\Lambda}(t) \hat{\rho}\left(\vec{k}_{1}\right) \hat{\Lambda}^{+}(t) \\
\left\langle m\left|\rho\left(\vec{k}_{1}, t\right)\right| m^{\prime}\right\rangle=\sum_{m_{a} m_{a}^{\prime}}\left\langle m|\Lambda(t)| m_{a}\right\rangle\left\langle m_{a}\left|\rho\left(\vec{k}_{1}\right)\right| m_{a}^{\prime}\right\rangle\left\langle m_{a}^{\prime}\left|\Lambda^{+}(t)\right| m^{\prime}\right\rangle
\end{gathered}
$$

Ahora, si $\mathrm{H}_{2}$ es el hamiltoniano de la transición $\mathrm{I} \rightarrow \mathrm{I}_{\mathrm{f}} \mathrm{y} \rho_{f}\left(\vec{k}_{1}, \vec{k}_{2}, t\right)$ es la matriz densidad del sistema luego de haber transcurrido la cascada $\gamma-\gamma$, entonces sus elementos de matriz serán [Frauenfelder, 1965]:

$$
\left.\left\langle m\left|\rho_{f}\left(\vec{k}_{1}, \vec{k}_{2}, t\right)\right| m^{\prime}\right\rangle=\sum_{m_{b} m_{b}^{\prime}}\left\langle m\left|H_{2}\right| m_{b}\right\rangle m_{b}\left|\rho\left(\vec{k}_{1}, t\right)\right| m^{\prime}{ }_{b}\right\rangle\left\langle m^{\prime}{ }_{b}\left|H_{2}^{+}\right| m^{\prime}\right\rangle
$$

La función de correlación angular es la traza de la matriz densidad $\rho_{f}\left(\vec{k}_{1}, \vec{k}_{2}, t\right)$ [Darriba, 2004]: 
$\left.W\left(\vec{k}_{1}, \vec{k}_{2}, t\right)=\sum_{\substack{m_{a} m^{\prime}, m_{b} m_{b}^{\prime}}}\left\langle m_{a}\left|\rho\left(\vec{k}_{1}\right)\right| m_{a}^{\prime}\right\rangle\left\langle m_{b}^{\prime}\left|\rho\left(\vec{k}_{2}\right)\right| m_{b}\right\rangle m_{b}|\Lambda(t)| m_{a}\right\rangle m_{b}^{\prime}|\Lambda(t)| m_{a}^{\prime}$

En la última expresión se pueden apreciar todos los procesos involucrados en la cascada $\gamma-\gamma$ : los elementos de matriz del operador evolución temporal contienen los efectos de la perturbación extranuclear; los elementos de matriz de las matrices densidad $\rho\left(\vec{k}_{1}\right)$ y $\rho\left(\vec{k}_{2}\right)$ están relacionados con la probabilidad de detectar $\gamma_{1}$ en la dirección $\vec{k}_{1}$ y $\gamma_{2}$ en la dirección $\vec{k}_{2}$, respectivamente.

Si reemplazamos en la ecuación (I.4) la forma explícita de los elementos de matriz de $\rho\left(\vec{k}_{1}\right)$ y $\rho\left(\vec{k}_{2}\right)$ [Rentería, 1987]:

$$
\begin{aligned}
& \left\langle m\left|\rho\left(\vec{k}_{1}\right)\right| m^{\prime}\right\rangle=\sqrt{4 \pi} \sum_{K_{1}, N_{1}}(-1)^{I+m} A_{K_{1}}\left(\gamma_{1}\right)\left(\begin{array}{ccc}
I & I & K_{1} \\
m^{\prime} & -m & N_{1}
\end{array}\right) Y_{K_{1}}^{N_{1}} *\left(\theta_{1}, \varphi_{1}\right) \\
& \left\langle m^{\prime}\left|\rho\left(\vec{k}_{2}\right)\right| m\right\rangle=\sqrt{4 \pi} \sum_{K_{2}, N_{2}}(-1)^{I+m^{\prime}} A_{K_{2}}\left(\gamma_{2}\right)\left(\begin{array}{ccc}
I & I & K_{2} \\
m & -m^{\prime} & N_{2}
\end{array}\right) Y_{K_{2}}^{N_{2}} *\left(\theta_{2}, \varphi_{2}\right)
\end{aligned}
$$

La forma explicita para la función de correlación angular queda [Darriba, 2004]:

$$
W\left(\vec{k}_{1}, \vec{k}_{2}, t\right)=4 \pi \sum_{K_{1}, K_{2}} A_{K_{1}}\left(\gamma_{1}\right) A_{K_{2}}\left(\gamma_{2}\right)\left[\left(2 K_{1}+1\right)\left(2 K_{2}+1\right)\right]^{-1 / 2} \sum_{N_{1} N_{2}} G_{K_{1} K_{2}}^{N_{1} N_{2}}(t) Y_{K_{1}}^{N_{1} *}\left(\theta_{1}, \varphi_{1}\right) Y_{K_{2}}^{N_{2}}\left(\theta_{2}, \varphi_{2}\right)
$$

donde $G_{K_{1} K_{2}}^{N_{1} N_{2}}(t)$ es el factor de perturbación y contiene la información de la interacción hiperfina, que usando (I.5) y (I.6) queda:

$$
\begin{aligned}
& G_{K_{1}, K_{2}}^{N_{1}, N_{2}}(t)=\sum_{m_{1}, m_{2}}(-1)^{2 I+m_{1}+m_{2}}\left[\left(2 K_{1}+1\right)\left(2 K_{2}+1\right)\right]^{1 / 2}\left(\begin{array}{ccc}
I & I & K_{1} \\
m_{1}^{\prime} & m_{1} & N_{1}
\end{array}\right)\left(\begin{array}{ccc}
I & I & K_{2} \\
m_{2}^{\prime} & m_{2} & N_{2}
\end{array}\right) \times \\
& \quad \times\left\langle m_{2}|\hat{\Lambda}(t)| m_{1}\right\rangle\left\langle m_{2}^{\prime}|\hat{\Lambda}(t)| m_{1}^{\prime}\right\rangle^{*}
\end{aligned}
$$

donde los símbolos $\left(\begin{array}{ccc}I & I & K_{1} \\ m_{1}^{\prime} & m_{1} & N_{1}\end{array}\right) y\left(\begin{array}{ccc}I & I & K_{2} \\ m_{2}^{\prime} & m_{2} & N_{2}\end{array}\right)$ son los símbolos 3-j de Wigner y se definen a partir de los coeficientes de Clebsch-Gordon por: 
$\left(\begin{array}{ccc}j_{1} & j_{2} & j_{3} \\ m_{1} & m_{2} & m_{3}\end{array}\right)=(-1)^{j_{1}-j_{2}-j_{3}}\left(2 j_{3}+1\right)^{-1 / 2}\left\langle j_{1} m_{1} j_{2} m_{2} \mid j_{3}-m_{3}\right\rangle$

Ahora dadas las autofunciones $\langle\vec{K} \sigma \mid \operatorname{Lm} \pi\rangle$, donde $\vec{K}$ y $\sigma$ son la dirección y polarización de una dada emisión y $L, m$ y $\pi$ son los autovalores de los operadores de momento angular, componente $\mathrm{z}$ del momento angular y paridad, respectivamente, definidas respecto de un eje $Z$ cuyo origen coincide con la fuente de radiación, vamos a relacionarlas con las mismas autofunciones pero en un sistema donde $\vec{K} \equiv Z$, y así ver el significado de los coeficientes 3-j de Wigner. Para esto se utilizan las matrices $D_{\mu m}^{L}(\vec{K} \rightarrow Z)$ del grupo de rotación tridimensional en la representación irreducible $D^{L}$ $2 L+1$ dimensional, de este modo:

$$
\langle\vec{K} \sigma \mid L m \pi\rangle=\sum\langle 0 \sigma| L \mu \pi D_{\mu m}^{L}(\vec{K} \rightarrow \vec{Z})
$$

Las matrices $D^{L}$ del grupo de rotación tridimensional cumplen las siguientes propiedades, de las cuales se deducen las propiedades de los símbolos 3-j:

$$
\begin{aligned}
& D_{\mu m}^{L}(\vec{K} \rightarrow \vec{Z})=D_{m \mu}^{L} *(\vec{Z} \rightarrow \vec{K}) \\
& D_{\mu m}^{L} D_{\mu^{\prime} m^{\prime}}^{L^{\prime}}=\sum_{K}\left\langle L \mu L^{\prime} \mu^{\prime} \mid K \tau\right\rangle\left\langle L m L^{\prime} m^{\prime} \mid K N\right\rangle D_{\tau N}^{K},
\end{aligned}
$$

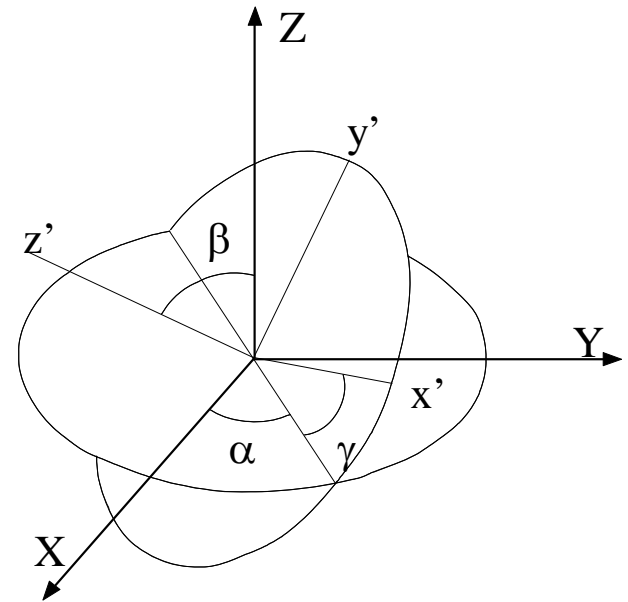

donde $K$ varía desde $/ L-L ' /$ hasta $L+L^{\prime}, N=m-m^{\prime}$ y $\tau$ $=\mu-\mu^{\prime}$. Y la forma explícita para $m=0$ y $\mu=0$ es:

$$
\begin{aligned}
& D_{\mu 0}^{L}(\alpha, \beta, \gamma)=\left(\frac{4 \pi}{2 L+1}\right)^{1 / 2} Y_{L}^{\mu *}(\alpha, \beta) \\
& D_{0 m}^{L}(\alpha, \beta, \gamma)=\left(\frac{4 \pi}{2 L+1}\right)^{1 / 2} Y_{L}^{-m}(\alpha, \beta),
\end{aligned}
$$

Figura I.1: Ángulos de Euler $\alpha, \beta$ y $\gamma$ que rota $\vec{K}$ al sistema $\mathrm{Z}$.

donde $\alpha, \beta$ y $\gamma$ son los ángulos de Euler que definen la rotación de un sistema Z’( $\vec{K}$ antes de la rotación) a un sistema Z (ver Figura I.1). 
A partir de las propiedades (I.14) de las matrices $D^{L}$, los símbolos 3-j cumplen:

$$
\begin{gathered}
\sum_{j_{3} m_{3}}\left(2 j_{3}+1\right)\left(\begin{array}{ccc}
j_{1} & j_{2} & j_{3} \\
m_{1} & m_{2} & m_{3}
\end{array}\right)\left(\begin{array}{ccc}
j_{1} & j_{2} & j_{3} \\
m_{1}^{\prime} & m_{2}^{\prime} & m_{3}^{\prime}
\end{array}\right)=\delta_{m_{1} m_{1}^{\prime}} \delta_{m_{2} m_{2}^{\prime}} \\
\sum_{m_{1} m_{2}}\left(2 j_{3}+1\right)\left(\begin{array}{ccc}
j_{1} & j_{2} & j_{3} \\
m_{1} & m_{2} & m_{3}
\end{array}\right)\left(\begin{array}{ccc}
j_{1} & j_{2} & j_{3}^{\prime} \\
m_{1} & m_{2} & m_{3}^{\prime}
\end{array}\right)=\delta_{j_{3} j_{3}^{\prime}} \delta_{m_{3} m_{3}^{\prime}} \\
\left(\begin{array}{ccc}
j_{1} & j_{2} & j_{3} \\
m_{1} & m_{2} & m_{3}
\end{array}\right)=\left(\begin{array}{ccc}
j_{2} & j_{3} & j_{1} \\
m_{2} & m_{3} & m_{1}
\end{array}\right)=\left(\begin{array}{ccc}
j_{3} & j_{1} & j_{2} \\
m_{3} & m_{1} & m_{2}
\end{array}\right) \\
(-1)^{j_{1}+j_{2}+j_{3}}\left(\begin{array}{ccc}
j_{1} & j_{2} & j_{3} \\
m_{1} & m_{2} & m_{3}
\end{array}\right)=\left(\begin{array}{ccc}
j_{2} & j_{1} & j_{3} \\
m_{2} & m_{1} & m_{3}
\end{array}\right)=\ldots
\end{gathered}
$$

Ahora volvamos al factor de perturbación (I.11) para simplificar tal expresión. Teniendo en cuenta que el GCE con el que interactúa el núcleo sonda en su estado intermedio I, no depende del tiempo, analizaremos la forma de la función de correlación perturbada y del factor de perturbación correspondiente a este tipo de interacción.

Por lo dicho en el párrafo anterior, $\mathrm{H}_{\mathrm{Q}}$ no depende del tiempo, de modo que el operador de evolución toma la forma:

$$
\Lambda(t)=e^{\frac{-i}{\hbar} H_{Q} t}
$$

En general, tanto $\Lambda(\mathrm{t})$ como $\mathrm{H}_{\mathrm{Q}}$ no son diagonales en la base $|m\rangle$, pero se expresan fácilmente en dicha base. Sea $\mathrm{U}$ la matriz que diagonaliza a $\mathrm{H}_{\mathrm{Q}}\left(\mathrm{E}=\mathrm{UH}_{\mathrm{Q}} \mathrm{U}^{-1}\right)$, de modo que la matriz E contenga en su diagonal los autovalores $E_{n}$, de modo que la exponencial de $\mathrm{H}_{\mathrm{Q}}$ diagonalizada queda:

$$
U e^{\frac{-i}{\hbar} H_{Q} t} U^{-1}=e^{\frac{-i}{\hbar} U H_{Q} U^{-1} t}=e^{\frac{-i}{\hbar} E t}
$$

Y asi el operador evolución nos queda: 


$$
\Lambda(t)=U^{-1} e^{\frac{-i}{\hbar} E t} U
$$

Entonces, la expresión para el factor de perturbación para el caso en que la interacción entre el campo extranuclear y el núcleo es independiente del tiempo nos queda:

$$
\begin{aligned}
& G_{K_{1}, K_{2}}^{N_{1}, N_{2}}(t)=\sum_{m_{1}, m_{2}, n, n^{\prime}}(-1)^{2 I+m_{1}+m_{2}}\left[\left(2 K_{1}+1\right)\left(2 K_{2}+1\right)\right]^{1 / 2}\left(\begin{array}{ccc}
I & I & K_{1} \\
m_{1}^{\prime} & m_{1} & N_{1}
\end{array}\right)\left(\begin{array}{ccc}
I & I & K_{2} \\
m_{2}^{\prime} & m_{2} & N_{2}
\end{array}\right) \times \\
& \times\left\langle n|\hat{\Lambda}(t)| m_{1}\right\rangle\left\langle n|\hat{\Lambda}(t)| m_{2}\right\rangle^{*}\left\langle n^{\prime}|\hat{\Lambda}(t)| m_{1}^{\prime}\right\rangle^{*}\left\langle n^{\prime}|\hat{\Lambda}(t)| m_{2}^{\prime}\right\rangle,
\end{aligned}
$$

donde se ha desarrollado el operador evolución temporal en la base de los subestados $|m\rangle$ y en la base $|n\rangle$ en que $\mathrm{H}_{\mathrm{Q}}$ es diagonal. De modo que en función de los autovalores $E_{n}$ la ecuación anterior queda:

$$
\begin{aligned}
& G_{K_{1}, K_{2}}^{N_{1}, N_{2}}(t)=\sum_{m_{1}, m_{2}, n, n^{\prime}}(-1)^{2 I+m_{1}+m_{2}}\left[\left(2 K_{1}+1\right)\left(2 K_{2}+1\right)\right]^{1 / 2}\left(\begin{array}{ccc}
I & I & K_{1} \\
m_{1}^{\prime} & m_{1} & N_{1}
\end{array}\right)\left(\begin{array}{ccc}
I & I & K_{2} \\
m_{2}^{\prime} & m_{2} & N_{2}
\end{array}\right) \times \\
& \quad \times\left\langle n \mid m_{1}\right\rangle\left\langle n \mid m_{2}\right\rangle^{*}\left\langle n^{\prime} \mid m_{1}^{\prime}\right\rangle^{*}\left\langle n^{\prime} \mid m_{2}^{\prime}\right\rangle e^{-i / \hbar\left(E_{n}-E_{n}^{\prime}\right) t}
\end{aligned}
$$

Si definimos:

$$
G_{K_{1} K_{2}}\left(\vec{k}_{1}, \vec{k}_{2}, t\right)=4 \pi\left[\left(2 K_{1}+1\right)\left(2 K_{2}+1\right)\right]^{-1 / 2} \sum_{N_{1} N_{2}} G_{K_{1} K_{2}}^{N_{1} N_{2}}(t) Y_{K_{1}}^{N_{1} *}\left(\theta_{1}, \varphi_{1}\right) Y_{K_{2}}^{N_{2}}\left(\theta_{2}, \varphi_{2}\right),
$$

Podemos expresar la función de correlación de una forma mucho más compacta:

$$
W\left(\vec{k}_{1}, \vec{k}_{2}, t\right)=\sum_{K_{1}, K_{2}} A_{K_{1} K_{2}} G_{K_{1} K_{2}}\left(\vec{k}_{1}, \vec{k}_{2}, t\right)
$$

donde $A_{K_{1} K_{2}}=A_{K_{1}}\left(\gamma_{1}\right) A_{K_{2}}\left(\gamma_{2}\right)$ es el producto entre la probabilidad de que se emita $\gamma_{1}$, de momento angular $K_{1}$ y la probabilidad de que se emita $\gamma_{2}$, de momento angular 
$\mathrm{K}_{2}$. Teniendo en cuenta que $K_{1}$ y $K_{2}$ no pueden superar el valor de 4 y que, como se ha mencionado más arriba, la probabilidad de transición entre los estados nucleares decrece al aumentar el momento angular de la radiación emitida, entonces, los $A_{K_{1} K_{2}}$ con $K_{1} \mathrm{y}$ $K_{2}$ mayores que 4 serán despreciables, y para los coeficientes diferentes de cero se tiene que $A_{22}>A_{24}, A_{42}, A_{44}$. Además, los únicos factores de perturbación no nulos son aquellos en que $\mathrm{K}_{1}=\mathrm{K}_{2}=\mathrm{K}$, por lo que (I.23) puede expresarse, vía el teorema de adición de los armónicos esféricos, como sigue:

$$
W(\theta)=\sum_{K=0}^{K_{\max }} A_{K K} G_{K K}(t) P_{K}(\cos (\theta))
$$

En ausencia de interacción hiperfina entre el núcleo-sonda y el campo extranuclear la función de correlación no perturbada queda:

$$
W(\theta)=\sum_{K=0}^{K_{\operatorname{maxx}}} A_{K K} P_{K}(\cos (\theta))
$$

\section{I-2 Función de correlación angular no perturbada para $I=1$}

Para fijar ideas analicemos el patrón de radiación de una correlación angular para momento de espín de estado intermedio $I=1$.

Consideremos la cascada mostrada en la Figura I.2, donde el momento angular de espín del estado inicial es $I_{i}=0$, el del estado intermedio es $I=1$ y el momento de espín del estado final es $I_{f}=0$; los momentos angulares de los cuantos $\gamma_{1}$ y $\gamma_{2}$ son $L_{1}=L_{2}=1$.
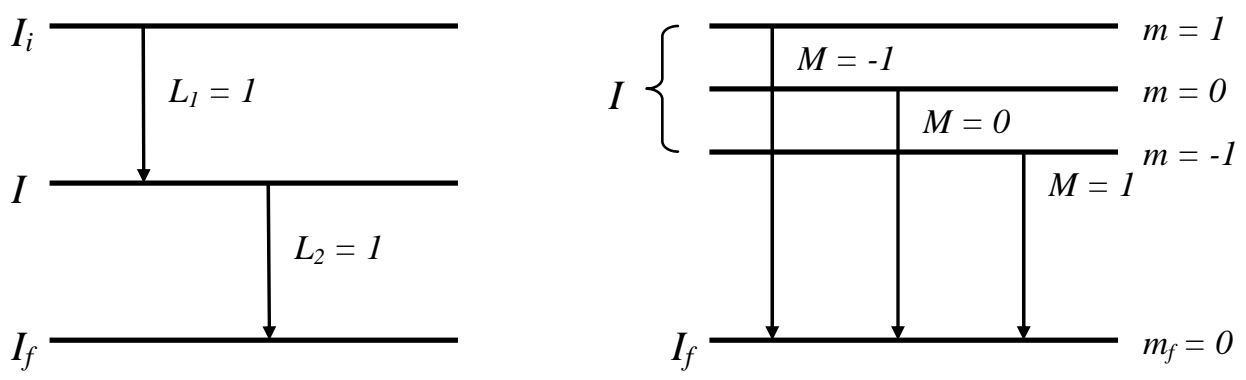

Figura I.2: Transiciones posibles del estado I al estado $\mathrm{I}_{\mathrm{f}}$ para el caso particular de $I_{i}=I_{f}=0 \mathrm{y} I=1$. 
Supondremos que los subniveles del estado intermedio $I$ y el estado inicial $I_{i}$ se encuentran uniformemente poblados. Primero consideraremos la transición esquematizada en la parte derecha de la Figura I.2. La distribución angular de la radiación dipolar emitida es función de $M=m_{f}-m$, que es la diferencia entre los números cuánticos magnéticos de los estados involucrados en la transición, en este caso el estado final y el estado intermedio, y que explícitamente queda expresada como:

$$
\begin{aligned}
& W(\theta) d \Omega=\frac{3}{8 \pi}\left(1-\cos ^{2} \theta\right) d \Omega \text { para } M=0, \\
& W(\theta) d \Omega=\frac{3}{16 \pi}\left(1+\cos ^{2} \theta\right) d \Omega \text { para } M=+1, \\
& W(\theta) d \Omega=\frac{3}{16 \pi}\left(1+\cos ^{2} \theta\right) d \Omega \text { para } M=-1,
\end{aligned}
$$

donde $\theta$ es el ángulo entre la dirección de emisión y un eje Z arbitrario de cuantificación. Es fácil ver que si las poblaciones son uniformes, la distribución angular deviene en isótropa, esto se consigue sumando las tres $W(\theta)$ de la expresión (I.26). Por lo tanto, para observar anisotropía en la distribución angular es necesario que los subestados magnéticos no estén igualmente poblados.

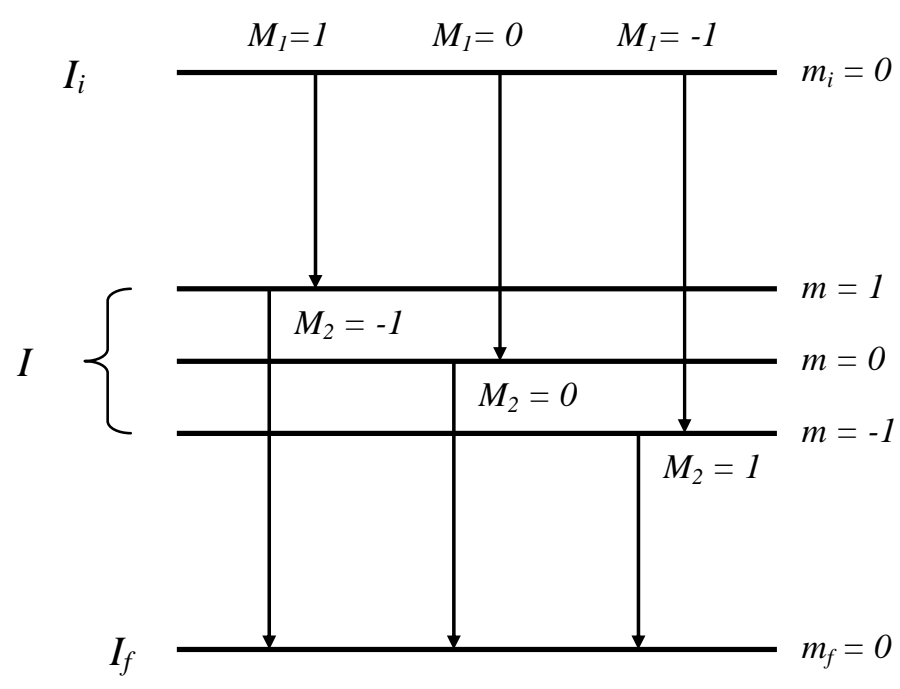

Figura I.3: cascada $\gamma-\gamma$ correspondiente a una interacción dipolar $(\mathrm{I}=1)$.
Consideremos, ahora si, la cascada $\gamma-\gamma$ para momento angular de espín del estado intermedio $I=1$. Esto se esquematiza en la figura I.3.

La dirección del eje de cuantificación $Z$ la tomaremos en la dirección de emisión de $\gamma_{1}$, esto es, $\theta=0$ coincide con la dirección $\vec{k}_{1}$. Entonces, por (I.26) la transición desde $M_{1}=0$ a $m=0$ es prohibida $(W(\theta)=0$ para $M=0$ ). Por lo que solo se 
producirán las transiciones $M_{1}=1$ seguida de $M_{2}=-1$ y $\quad M_{1}=-1$ seguida de $M_{2}=1$, ambas con la misma probabilidad proporcional a $\left(1+\cos ^{2} \theta\right)$.

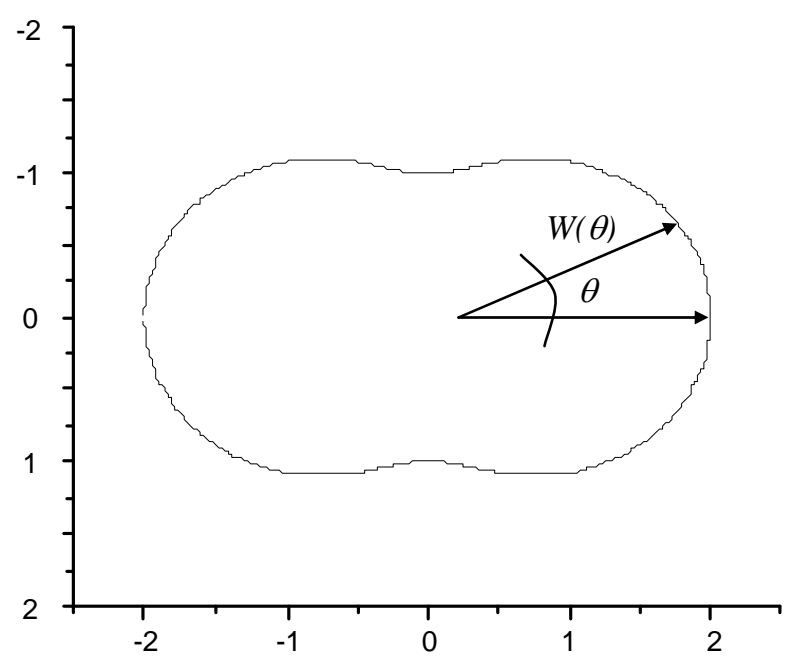

Figura I.4: Patrón de radiación de la Correlación angular debida a una radiación dipolar.
Por lo tanto, si el cuanto $\gamma_{1}$ se detecta en una dirección $\theta=0$, el cuanto $\gamma_{2}$ tendrá una distribución angular de emisión proporcional a $\left(1+\cos ^{2} \theta\right)$. En la Figura I.4 mostramos dicho patrón de emisión.

Al elegir una dirección arbitraria que la llamamos $Z$ y que es coincidente con la dirección de detección de $\gamma_{1}$, el cuanto $\gamma_{2}$ tendrá un patrón de emisión dipolar que en el plano de los detectores tendrá una forma similara la mostrada en la figura I.4, donde $\theta$ es el ángulo entre los detectores. Y para este caso de campo extranuclear nulo, dicho patrón permanecerá constante en el tiempo y tendrá una dependencia con el tiempo para campo extranuclear diferente de cero.

\section{Referencias}

[Rentería 1987] M. Rentería, Trabajo de Diploma, cap.1, ap.2, Departamento de Física, Facultad de Ciencias Exactas, Universidad Nacional de La Plata (1987).

[Frauenfelder, 1965] H. Frauenfelder y R. M. Steffen en “Alpha-Beta-and Gamma-Ray Spectoscopy”, vol.2 (North- Holland, Amsterdam 1968) P.997.

[Darriba, 2004] G. Darriba, Trabajo de Diploma, cap.3, Departamento de Física, Facultad de Ciencias Exactas, Universidad Nacional de La Plata (1987). 


\section{Apéndice II}

\section{II-1 Modelo de Bäverstam y Othaz}

Las interacciones hiperfinas dinámicas son producidas por cambios en las poblaciones de los estados atómicos excitados durante el tiempo transcurrido entre las emisiones de las radiaciones $\gamma_{1}$ y $\gamma_{2}$. Si se cumplen las siguientes condiciones:

(I) el tiempo de correlación $\tau_{c}$ es tal que $\omega_{Q} \tau_{c}<<1$, donde $\omega_{Q}$ es la frecuencia cuadrupolar eléctrica y $\tau_{c}$ un tiempo característico para los cambios producidos en el entorno del núcleo-sonda;

(II) el tiempo de observación es tal que $t>>\tau_{c}$;

(III) la intensidad de la interacción dinámica es lo suficientemente pequeña de modo que es válida la teoría de perturbaciones a primer orden.

Entonces, el Factor de Perturbación Dinámico puede expresarse como [Abragam 1953]:

$G_{22}^{d}(t)=e^{-\lambda_{r} t}$

donde $\lambda_{\mathrm{r}}$ es la constante de relajación dinámica.

Sin embargo, esta expresión no tiene en cuenta el hecho que determinados átomos excitados tienen una probabilidad no nula de alcanzar el estado fundamental antes de haber emitido la segunda radiación. Para considerar este efecto, Bäverstam y Othaz hacen dos simplificaciones: (I) la probabilidad por átomo de alcanzar el estado fundamental luego de transcurrido un tiempo $t$ es:

$$
P_{g}(t)=\lambda_{g} e^{-\lambda_{g} t}=\tau_{g}^{-1} e^{-t / \tau_{g}}
$$

donde $\lambda_{\mathrm{g}}$ es la constante de recuperación atómica;

(II) la intensidad de la interacción dinámica promediada sobre todos los átomos excitados en constante $\left(\tau_{r}=1 / \lambda_{r}=\right.$ cte.) [Bäverstam 1972]. Para el caso en que los átomos hayan alcanzado el estado fundamental, la interacción dinámica será despreciable (o nula). 
El factor de perturbación al tiempo $t$ (en que se detecta la radiación $\gamma_{2}$ ) tiene dos contribuciones: la primer contribución está dada por el factor de perturbación estático promediado, pesado por los núcleos que ya decayeron al estado fundamental (GCE final estable) antes de $t$; y la segunda contribución viene dada por el factor estático multiplicado por $\exp \left(-\lambda_{r} t\right)$ pesado por las sondas que decaerán luego de $t$ (es decir por el complemento de las que ya decayeron). De este modo, el factor dinámico toma la forma:

$$
G_{22}^{d}(t)=\int_{0}^{t} \lambda_{g} e^{-\lambda_{g} t^{\prime}} e^{-\lambda_{r} t^{\prime}} d t^{\prime}+e^{-\lambda_{r} t} \int_{t}^{\infty} \lambda_{g} e^{-\lambda_{g} t^{\prime}} d t^{\prime}=\frac{\lambda_{g}}{\lambda_{g}+\lambda_{r}}+\frac{\lambda_{r}}{\lambda_{g}+\lambda_{r}} e^{-\left(\lambda_{g}+\lambda_{r}\right) t}
$$

Entonces, el factor de perturbación nos queda:

$$
G_{22}(t)=G_{22}^{s}(t) \cdot G_{22}^{d}(t)=\frac{\lambda_{g}}{\lambda_{g}+\lambda_{r}} \cdot G_{22}^{s}(t)+\frac{\lambda_{r}}{\lambda_{g}+\lambda_{r}} e^{-\left(\lambda_{g}+\lambda_{r}\right) t} \cdot G_{22}^{s}(t)
$$

\section{Referencias}

[Abragam, 1953] A. Abragam and R. V. Pound, Phys. Rev. 92, 943 (1953).

[Bäverstam, 1972] U. Bäverstam, R. Othaz, N. De Sousa and B. Ringström, Nucl. Phys. A186, 500 (1972). 


\section{Apéndice III}

\section{III-1 Cálculos PCM en óxidos con estructura Bixbita}

En la Tabla III.1 se muestran los parámetros hiperfinos predichos en el marco del Modelo de Cargas Puntuales (PCM) para el caso de celda no relajada, es decir, los sitios atómicos fueron determinados a partir de parámetros estructurales experimentales. Como vimos en el capítulo $\mathrm{N}^{\circ} 2$, en el modelo PCM calculamos el tensor GCE en el sitio catiónico de la sonda Cd a partir de las contribuciones de cargas puntuales ubicadas en los sitios de la red. De este modo, el valor de una dada carga es igual a la valencia del átomo en cuestión. En el caso de las Bixbitas la carga de los sitios catiónicos es +3 (igual a la valencia del catión) mientras que la carga de los sitios de los oxígnos es -2. Finalmente, la contribución de los electrones del Cd al tensor GCE se tiene en cuenta a partir del factor de apantallamiento de Sternheimer $\gamma_{\infty}$ (para el caso del Cd $\gamma_{\infty}=-29,27$ ), por lo que la componente principal del tensor toma la forma que vimos en la ecuación (2.4):

$$
V_{33}=\left(1-\gamma_{\infty}\right) V_{33}^{\text {red }}
$$

\begin{tabular}{|c|c|c|c|c|c|c|c|c|c|c|}
\hline \multirow[t]{2}{*}{ Sistema } & \multicolumn{5}{|c|}{ Sitio $D$} & \multicolumn{5}{|c|}{ Sitio $C$} \\
\hline & $\boldsymbol{d}_{N N}$ & $V_{33}{ }^{r e d}$ & $V_{33}$ & $\eta$ & Dir $V_{33}$ & $\boldsymbol{d}_{N N}$ & $V_{33}^{r e d}$ & $V_{33}$ & $\eta$ & Dir $V_{33}$ \\
\hline $\operatorname{In}_{2} \mathrm{O}_{3}$ & 2.19 & 0.25 & 7.54 & 0.00 & {$\left[\begin{array}{lll}1 & 1 & 1\end{array}\right]$} & $\begin{array}{l}2.12 \\
2.19 \\
2.21\end{array}$ & -0.16 & -4.87 & 0.82 & {$\left[\begin{array}{lll}1 & -0.8 & 0\end{array}\right]$} \\
\hline $\mathbf{Y}_{2} \mathbf{O}_{3}$ & 2.28 & 0.26 & 7.75 & 0.00 & {$\left[\begin{array}{lll}1 & 1 & 1\end{array}\right]$} & $\begin{array}{l}2.24 \\
2.27 \\
2.33\end{array}$ & -0.12 & -3.51 & 0.63 & {$\left[\begin{array}{lll}-0.6 & 1 & 0\end{array}\right]$} \\
\hline $\mathrm{Sc}_{2} \mathrm{O}_{3}$ & 2.12 & 0.23 & 7.05 & 0.00 & {$\left[\begin{array}{lll}1 & 1 & 1\end{array}\right]$} & $\begin{array}{l}2.08 \\
2.12 \\
2.16\end{array}$ & -0.12 & -3.81 & 0.95 & {$\left[\begin{array}{lll}1 & -0.6 & 0\end{array}\right]$} \\
\hline
\end{tabular}

Tabla III.1: Resultados PCM para los óxidos con estructura Bixbita utilizando parámetros de red experimentales (celda no relajada). $d_{N N}$ se expresa en $\AA$ y $V_{33}$ en unidades de $10^{21} \mathrm{~V} / \mathrm{m}^{2}$.

En la Tabla III.2 se muestran los resultados PCM que caracterizan el tensor GCE en el sitio del Cd para el caso de celda relajada. Las posiciones relajadas fueron obtenidas a 
partir del método FPAPW+lo para el caso cargado debido a que a altas temperaturas las asignaciones realizadas corresponden a dicho estado de carga de la impureza.

\begin{tabular}{|c|c|c|c|c|c|c|c|c|c|c|}
\hline \multirow[t]{2}{*}{ Sistema } & \multicolumn{5}{|c|}{ Sitio $D$} & \multicolumn{5}{|c|}{ Sitio C } \\
\hline & $\boldsymbol{d}_{N N}$ & $V_{33}^{\text {red }}$ & $V_{33}$ & $\eta$ & Dir. $V_{33}$ & $\boldsymbol{d}_{N N}$ & $V_{33}^{\text {red }}$ & $V_{33}$ & $\eta$ & Dir. $V_{33}$ \\
\hline $\operatorname{In}_{2} \mathrm{O}_{3}$ & 2.28 & 0.23 & +6.95 & 0.00 & {$\left[\begin{array}{lll}1 & 1 & 1\end{array}\right]$} & $\begin{array}{l}2.20 \\
2.30 \\
2.32\end{array}$ & 0.17 & +5.11 & 0.63 & {$\left[\begin{array}{lll}0.7 & 1 & 0\end{array}\right]$} \\
\hline $\mathbf{Y}_{2} \mathbf{O}_{3}$ & 2.36 & 0.23 & +6.95 & 0.00 & {$\left[\begin{array}{lll}1 & 1 & 1\end{array}\right]$} & $\begin{array}{l}2.27 \\
2.35 \\
2.45\end{array}$ & 0.12 & +3.97 & 0.67 & {$\left[\begin{array}{lll}1 & 0.6 & 0\end{array}\right]$} \\
\hline $\mathrm{Sc}_{2} \mathrm{O}_{3}$ & 2.28 & 0.22 & +6.82 & 0.00 & [ [ $\left.\begin{array}{lll}1 & 1 & 1\end{array}\right]$ & $\begin{array}{l}2.19 \\
2.31 \\
2.32 \\
\end{array}$ & 0.16 & +5.28 & 0.57 & {$\left[\begin{array}{lll}0.9 & 1 & 0\end{array}\right]$} \\
\hline
\end{tabular}

Tabla III.2: Resultados PCM para los óxidos con estructura Bixbita utilizando relajaciones estructurales predichas por el método FPAPW+lo (celda relajada). $d_{N N}$ se expresa en $\AA$ y $V_{33}$ en unidades de $10^{21} \mathrm{~V} / \mathrm{m}^{2}$.

\section{III-2 Cálculos PCM en los óxidos $\mathrm{ZnO}$, $\mathrm{SnO}$ y $\mathrm{SnO}_{2}$}

En la Tabla III.3 se muestran los parámetros hiperfinos calculados con PCM para el caso de celda no relajada (coordenadas experimentales) y celda relajada (relajaciones predichas por FPAPW+lo). Teniendo en cuenta los estados de carga asignados a las interacciones hiperfinas observadas, se eligieron las distorsiones estructurales que están en acuerdo con estas asignaciones.Por lo tanto, para los casos de $\mathrm{ZnO}$ y $\mathrm{SnO}$ se utilizan las relajaciones correspondientes a la celda neutra mientras que para $\mathrm{SnO}_{2}$ las relajaciones de celda cargada.

\begin{tabular}{|c|c|c|c|c|c|c|c|c|c|c|}
\hline \multirow[t]{2}{*}{ Sistema } & \multicolumn{5}{|c|}{ Celda no relajada } & \multicolumn{5}{|c|}{ Celda relajada } \\
\hline & $\boldsymbol{d}_{N N}$ & $V_{33}{ }^{\text {red }}$ & $V_{33}$ & $\eta$ & Dir. $V_{33}$ & $\boldsymbol{d}_{N N}$ & $V_{33}{ }^{\text {red }}$ & $V_{33}$ & $\eta$ & Dir. $V_{33}$ \\
\hline $\mathrm{ZnO}$ & $\begin{array}{l}1.97 \\
1.99 \\
\end{array}$ & 0.03 & 0.94 & 0.00 & {$\left[\begin{array}{lll}0 & 0 & 1\end{array}\right]$} & $\begin{array}{l}2.16 \\
2.19 \\
\end{array}$ & 0.01 & 0.45 & 0.00 & {$\left[\begin{array}{lll}0 & 0 & 1\end{array}\right]$} \\
\hline $\mathrm{SnO}$ & 2.22 & 0.18 & 5.45 & 0.00 & {$\left[\begin{array}{lll}0 & 0 & 1\end{array}\right]$} & 2.31 & 0.12 & 3.69 & 0.00 & {$\left[\begin{array}{lll}0 & 0 & 1\end{array}\right]$} \\
\hline $\mathrm{SnO}_{2}$ & $\begin{array}{l}2.05 \\
2.06\end{array}$ & 0.17 & 5.21 & 0.40 & {$\left[\begin{array}{lll}1 & -1 & 0\end{array}\right]$} & $\begin{array}{l}2.15 \\
2.20\end{array}$ & 0.18 & -5.56 & 0.95 & {$\left[\begin{array}{lll}1 & -1 & 0\end{array}\right]$} \\
\hline
\end{tabular}

Tabla III.3: Resultados PCM para los óxidos $\mathrm{SnO}, \mathrm{ZnO}$ y $\mathrm{SnO}_{2}$ para los casos de celda no relajada y celda relajada. $d_{N N}$ se expresa en $\AA$ y $V_{33}$ en unidades de $10^{21} \mathrm{~V} / \mathrm{m}^{2}$. 


\section{Publicaciones}

Trabajos publicados a partir de resultados presentados en esta Tesis:

1- TDPAC study of Cd-doped SnO

E. L. Muñoz, A. W. Carbonari, L. A. Errico, A.G. Bibiloni , H. M. Petrilli and M. Rentería.

Hyperfine Interactions 178, 37 (2007).

2- $A b$ initio study of the EFG tensor at $\mathrm{Cd}$ impurities in $\mathrm{Sc}_{2} \mathrm{O}_{3}$ semiconductor E.L. Muñoz, D. Richard, L.A. Errico and M. Rentería. Physica B 404, 2757 (2009).

3- PAC study of the dynamic hyperfine interactions at ${ }^{111} \mathrm{In}$-doped $\mathrm{Sc}_{2} \mathrm{O}_{3}$ semiconductor and comparison with ab initio calculations E. L. Muñoz, D. Richard, A. W. Carbonari, L. A. Errico and M. Rentería. Hyperfine Interactions 197, 199 (2011).

4- Efficiency-Optimized Low-Cost TDPAC Spectrometer Using a Versatile Routing/Coincidence Unit

M. Rentería, A.G. Bibiloni, G.N. Darriba, L.A. Errico, E.L. Muñoz, D. Richard and J. Runco.

Hyperfine Interactions 181, 145 (2008).

5- Electronic and structural properties and hyperfine interactions at Sc sites in the semiconductor $\mathrm{Sc}_{2} \mathrm{O}_{3}$ : TDPAC and $a b$ initio study

D. Richard, E. L. Muñoz, T. Butz, L. A. Errico, and M. Rentería.

Physical Review B 82, 035206 (2010). 
6- Dynamic hyperfine interactions in ${ }^{111} \mathrm{In}\left({ }^{111} \mathrm{Cd}\right)$-doped $\mathrm{ZnO}$ semiconductor: PAC results supported by ab initio calculations

E.L. Muñoz, M.E. Mercurio, M.R. Cordeiro, L.F.D. Pereira, A.W. Carbonari and M. Rentería.

(Enviado para su publicación a Physica B (2010)).

Otros trabajos publicados realizados en temas afines a partir del doble abordaje experimental - $a b$ initio durante mi carrera de doctorado:

1- Electric-Field Gradient Characterization at ${ }^{181}$ Ta Impurities in Sapphire Single Crystals

M. Rentería, G. N. Darriba, L. A. Errico, E. L. Muñoz, and P. D. Eversheim.

Physica Status Solidi b 242, 1928 (2005).

2- Ionic Exchange of Hf Donor Impurities in the Wide-Gap Semiconductor $\mathrm{Tm}_{2} \mathrm{O}_{3}$

E.L. Muñoz, G.N. Darriba, A.G.Bibiloni, L.A.Errico, and M. Rentería. Journal of Alloys and Compounds 495, 532 (2010).

3- PAC and PALS study in the $\mathrm{HfO}_{2}+\mathrm{SiO}_{2}$ system

C. Y. Chain, L. C. Damonte, S. Ferrari, E. L. Muñoz, C. Rodríguez Torres and A. F. Pasquevich.

Journal of Alloys and Compounds 495, 527 (2010).

4- Electric-Field Gradients at Ta donor impurities in $\mathrm{Cr}_{2} \mathrm{O}_{3}(\mathrm{Ta})$ semiconductor

G.N. Darriba, L.A. Errico, E.L. Muñoz, D. Richard, P.D. Eversheim amd M. Rentería.

Physica B 404, 2739 (2009).

5- Electronic and structural properties of the $\alpha-\mathrm{Fe}_{2} \mathrm{O}_{3}$ (Ta) semiconductor Experimental EFG determination and $a b$ initio calculations

G. N. Darriba, E. L. Muñoz, P. D. Eversheim and M. Rentería.

Hyperfine Interactions 197, 207 (2011). 
6- Experimental and $a b$ initio study of Ta-doped $\mathrm{ZnO}$ semiconductor

E. L. Muñoz, D. Richard, P.D Eversheim and M.Rentería.

Hyperfine Interactions 197, 181 (2011).

Presentaciones a congresos relacionadas con mi Trabajo de Tesis:

1- A first-principles model for electron-capture "after-effects" in ${ }^{111} \mathrm{Cd}$ : $\operatorname{In}_{2} \mathrm{O}_{3}$ M. Rentería, L. A. Errico, E. L. Muñoz and A. G. Bibiloni.

Evento: At the Frontiers of Condensed Matter III, Buenos Aires, 11 al 15 de diciembre de 2006.

Carácter de participación: presentación de póster.

2- Study of hyperfine interactions in ${ }^{111} \mathrm{Cd}$-doped $\mathrm{SnO}$ semiconductor with PAC measurement and $a b$ initio calculations

E. L. Muñoz, A. W. Carbonari, L. A. Errico, H. M. Petrilli and M. Rentería. Evento: XIV International Conference on Hyperfine Interactions and XVIII International Symposium on Nuclear Quadrupole Interactions, Foz Iguazú, 5 al 10 de agosto de 2007.

Carácter de participación: presentación de Póster

3- Time-Differential Perturbed-Angular Correlations and $a b$ initio Study of Cd-doped SnO Semiconductor

E. L. Muñoz, A. W. Carbonari, L. A. Errico, A. G. Bibiloni, H. M. Petrilli and M. Rentería.

Evento: 29th International Conference on the Physics of Semiconductors, Río de Janeiro, 27 de Julio al 1 de agosto de 2008.

Carácter de participación: presentación de póster.

4- $A b$ initio study of $\mathrm{Cd}$-doped $\mathrm{Sc}_{2} \mathrm{O}_{3}$ semiconductor and comparison with ${ }^{111}$ Cd PAC experiments

E.L. Muñoz, D. Richard, L.A. Errico and M. Rentería.

Evento: At the Frontiers of Condensed Matter IV, Buenos Aires, 9 al 12 de diciembre de 2008.

Carácter de participación: presentación de póster. 
5- PAC study of the dynamic hyperfine interactions at ${ }^{111} \mathrm{In}$-doped $\mathrm{Sc}_{2} \mathrm{O}_{3}$ semiconductor and comparison with ab initio calculations

E. L. Muñoz, D. Richard, A. W. Carbonari, L. A. Errico and M. Rentería. Evento: HFI/NQI2010, CERN Ginebra, 12 al 17 de septiembre de 2010. Carácter de participación: Presentación Oral.

6- Search for “After-Effects” in Cd-doped ZnO semiconductor: PAC experiments supported by ab initio results

E. L. Muñoz, M. Mercurio, A. W. Carbonari and M. Rentería. Evento: HFI/NQI2010, CERN Ginebra, 12 al 17 de septiembre de 2010. Carácter de participación: Presentación de póster.

7- Study of electronic structure and electric gradient field at Cd probe in Codoped ZnO matrix by first principles calculations

Autores: L. F. D. Pereira, E. L. Muñoz, A. W. Carbonari, M. Cordeiro, M. Mercúrio, M. Rentería and J. Mestnik-Filho.

Evento: HFI/NQI2010, CERN Ginebra, 12 al 17 de septiembre de 2010 Carácter de participación: Presentación de póster. 


\section{Agradecimientos}

En primer lugar deseo agradecer la financiación de este trabajo brindada por la UNLP, por el CONICET y por el Instituto de Física La Plata (IFLP, CONICET-UNLP). En particular, al CONICET que me becó durante mi carrera de doctorado.

Deseo agradecer la formación brindada por Mario Rentería en estos cinco años de trabajo. La libertad brindada a la hora de elegir el tema de Tesis y su apoyo incondicional en Argentina y, en particular, en mis estadías en el exterior. A los otros integrantes del grupo (Ger, el Colo y Leo) por aceptarme tal como soy. En especial a Ger y al Colo por todo lo compartido durante esta etapa, por las charlas de física, por las cervezas, por los mates, por las benditas empanadas de Don Onorio que momentáneamente hicieron tambalear la escritura de esta Tesis.

A los Profesores Dra. Helena M. Petrilli, Dr. Artur W. Carbonari y Dra. Graciela Punte por haber aceptado evaluar mi trabajo de Tesis.

Al Profesor Dr. Anibal G. Bibiloni por las charlas compartidas acerca del modelo “del hueco atractor” y por darme el empujón a realizar un nuevo modelo con la novedad de los cálculos ab initio.

Al Prof. Dr. Alejandro Ayala por haber modificado el programa de ajuste TDPAC para poder ajustar espectros PAC con atenuación dinámica a partir del modelo de Bäverstam y Othaz en forma independiente para cada interacción.

Al Prof. Dr. Jorge A. Martinez (Toto) por haber modificado el programa CdCPAC para poder ajustar espectros PAC con interacciones dinámicas a partir del modelo de Bäverstam y Othaz.

A Artur W. Carbonari por haberme recibido en su laboratorio, por su predisposición para realizar los experimentos y por escuchar cada pedido mío de manera incondicional durante mi estadía.

Al Dr. Rakesh Dogra por su mano brindada en los experimentos (particularmente los fines de semana).

A Helena M. Petrilli por sus cordiales charlas en mi estadía en la USP. En particular, deseo agradecer a Ney por haberme alojado en su morada en dicha estadía. 
Deseo agradecer al Program Committee del HFI\&NQI 2010 (CERN, Ginebra, Suiza) por brindarme la posibilidad de exponer el modelo presentado en esta Tesis y parte de sus resultados en una contribución oral en el auditorio Main Auditorium frente a la comunidad de Interacciones Hiperfinas.

A Luciano F. Pereira por su apoyo en la realización de algunos cálculos hechos durante mi estadía en la USP y por sus “interminables” charlas.

A los becarios del IPEN y del Dpto. de Física de la USP por gratificar mis días en la USP.

Deseo agradecer al Prof. Dr. Alberto Pasquevich por la organización y el dictado del curso de interacciones hiperfinas (mitad en la UNLP y mitad en Río de Janeiro). Además, deseo agradecer a Vitaly, Sergio, Rúben y Azucena por aguantarme en Río, en particular, a Rubén por compartir algunas cervezas en las noches de Copacabana a pesar de su gastritis.

Deseo agradecer al Laboratorio de Electrónica del Dpto. de Física y, en particular, al Prof. Ing. Jorge Runco por la asistencia brindada para el correcto funcionamiento del espectrómetro PACar en experimentos realizados con la sonda ${ }^{181} \mathrm{Hf} \rightarrow{ }^{181}$ Ta durante mi carrera de doctorado.

Deseo agradecer a toda mi familia Chivilcoyana por su incondicional apoyo en cualquier tiempo y lugar. En especial a mi vieja que ante cualquier llamado se tomaba el TALP para venir a cuidar a Santi.

Deseo agradecer a mi suegra y mis cuñados, en especial, a Lore por todo lo compartido.

A José, Pome y Diego por la amistad que nos une desde hace años y se fortalece con el paso del tiempo. Tampoco me quiero olvidar de la fiera y los mates compartidos en la garita de Odontología (y las charlas de filosofía de la ciencia).

Deseo agradecer a la gente de Física III de Ingeniería por los momentos compartidos, en especial a Porcel y Mariano por los mates y las charlas de los miércoles y viernes a las 8 a.m.

Finalmente deseo agradecer a las dos personas que me "bancaron” pese a todo día y noche durante estos cinco años. Una de ellas por decisión propia y la otra persona por “necesidad biológica”. Gracias Gorda y Santi por aceptarme especialmente en mis momentos de raye. 